Adoption and implemention of smoking cessation support in health care 


\section{Promotor:}

Prof. Dr. H. de Vries

\section{Copromotores:}

Dr. C. Bolman (Open Universiteit Nederland, Heerlen)

Dr. M.C. Willemsen (STIVORO voor een rookvrije toekomst, Den Haag)

\section{Beoordelingscommissie:}

Prof. dr. N. K. de Vries (voorzitter)

Prof. dr. H.W. van den Borne

Dr. I. Mesters

Dr. Th. Paulussen (TNO Preventie en Gezondheid, Leiden)

\section{Colofon}

Foto omslag: Tarquinia Zeegers @

Druk: Repro Facility NRG

ISBN-10: 90-9021148-9

ISBN-13: 978-90-9021148-0

Copyright (c) 2006 Dewi Segaar, Haarlem

The studies described in this thesis were partly supported by a grant of ZonMwthe Netherlands Organization for Health Research and Development (2500.0007 and 2500.0008) and from STIVORO for a smokefree future. Financial support by the Netherlands Heart Foundation and by STIVORO for a smokefree future for the publication of this thesis is gratefully aknowledged. 


\title{
Adoption and implemention of smoking cessation support in health care
}

\author{
Proefschrift
}

ter verkrijging van de graad van doctor aan de Universiteit Maastricht op gezag van de Rector Magnificus, Prof. mr. G.P.M.F. Mols, volgens het besluit van het College van Decanen, in het openbaar te verdedigen op woensdag 20 december 2006 om 16.00 uur door Dewi Segaar 



\section{Contents}

\section{Chapter 1}

General introduction

Chapter 2

Determinants of adoption of cognitive behavioral interventions in a hospital setting: example of a minimal-contact smoking cessation intervention for cardiology wards

\section{Chapter 3}

Characteristics of adherence to a minimal-contact smoking cessation

intervention on cardiac wards

Chapter 4

Identifying determinants of protocol adoption by midwives: a

comprehensive approach

\section{Chapter 5}

Adherence to a minimal-contact smoking cessation intervention by midwives

Chapter 6

Determinants of midwifery practices' implementation of an intervention for

smoking cessation support by midwives

Chapter 7

Adoption and implementation of smoking cessation support in two health

care settings

Chapter 8

General discussion

References

Summary

Samenvatting

Dankwoord 



\section{CHAPTER 1}

General Introduction

Wees de verandering die je in de wereld wil zien.

(Mahatma Gandhi) 


\subsection{Background}

Worldwide, massive amounts of money are being spent on healthcare. In the Netherlands alone, healthcare expenditure amounts to some 48 billion euros a year (Tweede Kamer de Staten Generaal, 2005), corresponding to about $10 \%$ of the Gross Domestic Product (GDP). The corresponding figure in the U.S. is even higher: $24 \%$ of the GDP (or 1.9 trillion dollars). The responsibility for the efficient spending of this money lies especially with the healthcare providers, who have to provide patients with the right care at the right moment. This is a difficult job, as the number of available treatments is immense. Therefore, multidisciplinary groups, including expert clinicians, health service researchers and other relevant experts develop clinical practice guidelines to support healthcare providers in their decisions on what care to provide and how to provide it (Shekelle, Woolf, Eccles, \& Grimshaw, 1999).

Clinical practice guidelines are systematically developed statements to assist practitioner and patient decisions about appropriate healthcare for specific clinical circumstances (Institute of Medicine, 1990). These guidelines are evidence-based, they support medical practice to an important extent and they contribute to quality assurance of medical actions. The National Guideline Clearinghouse ${ }^{\mathrm{TM}}$ (NGC), a public resource for clinical practice guidelines in the U.S., has registered 1772 guidelines that meet the following criteria: evidence-based, developed or updated in the last 5 years and fully available in the English language. In the Netherlands, the Dutch Institute for Healthcare Improvement ( $\mathrm{CBO}$ ) developed 50 evidence-based clinical practice guidelines between 2000 and 2005.

Although there is thus no lack of guidelines, the problem is how to diffuse these guidelines to daily practice (Cranney, Warren, Barton, Gardner, \& Walley, 2001; Grol \& Grimshaw, 2003). Diffusion is the process by which an innovation is communicated through certain channels over time among the members of a social system (Rogers, 1995). Guidelines, whether or not translated into more concrete protocols or pathways, tend to suffer from underuse and inappropriate use, as has been found in several countries (Davis \& Taylor-Vaisey, 1997; Grol, 2001; Katz, Brown, Muehlenbruch, Fiore, \& Baker, 2004) including the Netherlands (Grol, 2001). Several international studies suggest that at least one third and probably even more of the patients in developed countries do not receive the care they should receive according to scientific evidence or evidence-based guidelines (Schuster, McGlynn, \& Brook, 2005; Seddon, Marshall, Campbell, \& Roland, 2001). No exact data on the Dutch situation are available, but a national study into the quality of care in general practices revealed that guideline adherence differs between guidelines, ranging from as little as $10 \%$ to almost $100 \%$. The mean proportion of general practitioners who act in accordance with relevant guidelines was $68 \%$ (Braspenning, Schellevis, \& Grol, 2004). 
The effectiveness of health promotion interventions, such as clinical guidelines and protocols, and the success of their diffusion are highly dependent on the quality of their planning (Green \& Kreuter, 1999; Kok, Van den Borne, \& Mullen, 1997). Therefore, several planning models for health promotion interventions have been developed. One of them is the I-PLAN or ABC-planning model (De Vries, 1998; De Vries \& Kok, 1996), which can be viewed as a comprehensive model for systematically planning, developing and diffusing health education and promotion activities. This model integrates several planning models that have been used in the field of health education and promotion, building, for example, on the Precede-Proceed framework (Green \& Kreuter, 1999) and Rogers' Diffusion of Innovations Theory (Rogers, 1995). The model is shown in Figure 1 and distinguishes three basic phases in the process of health education and promotion: Analysis of the problem, Behavioral change and Continuation.

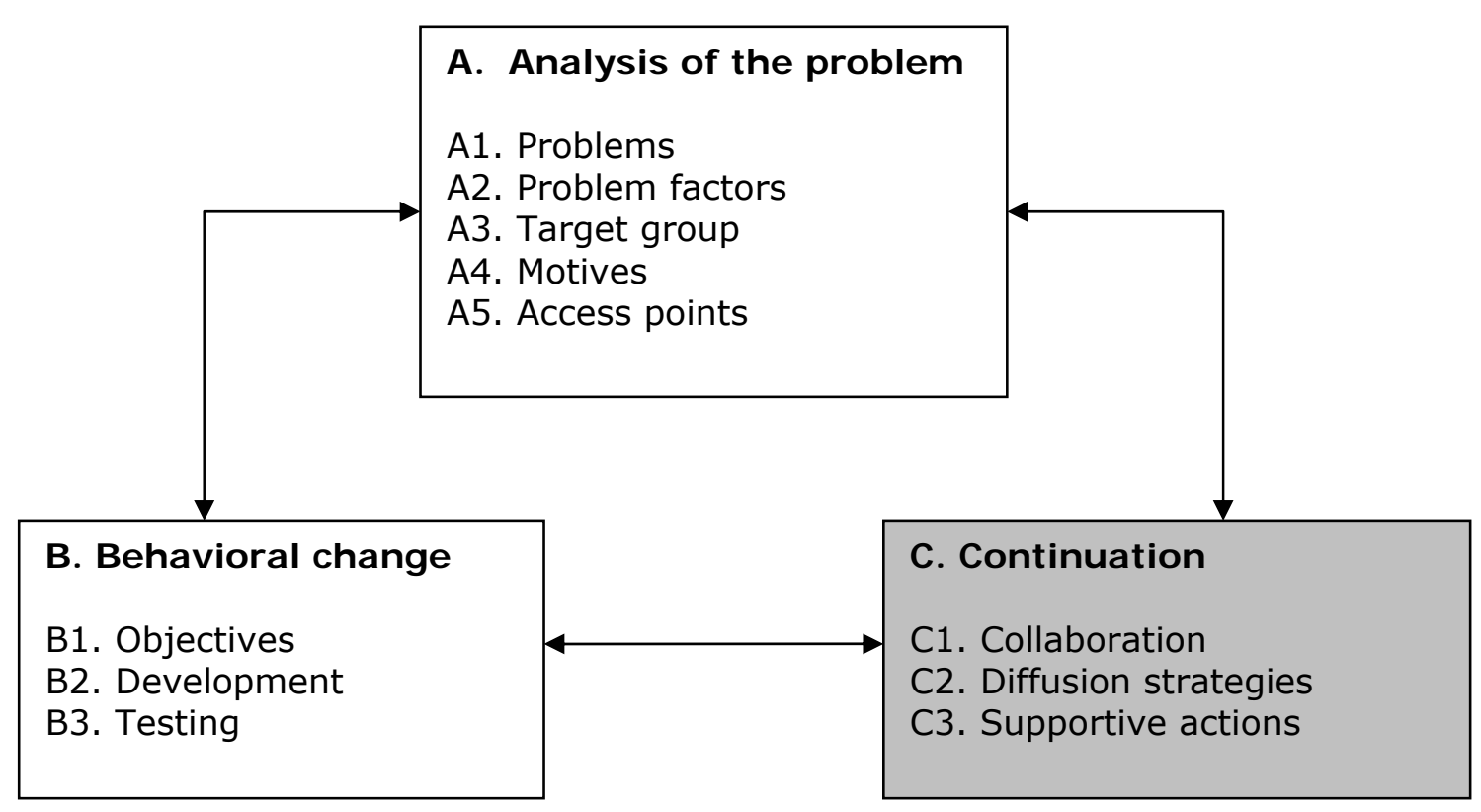

Figure 1. The I-PLAN model (De Vries, 1998; De Vries \& Kok, 1996)

In the analysis phase, the problem and behaviors related to the problem are identified, a target group is defined and the factors that determine a person's behavior are analyzed. In the second phase, a choice is made about ways to change the behavior, intervention goals are set and a program is developed and tested. In the third phase, diffusion strategies are developed, intersectoral collaboration and support is established and supportive policies are developed. In the systematic planning process of most interventions, policymakers tend to give much attention to phases $A$ and $B$, and a great deal of research is performed during these phases. The continuation phase, which is also often named diffusion or dissemination phase, is however often neglected by both 
policymakers and researchers. Diffusion research constitutes less than $10 \%$ of all public health and health promotion research (Oldenburg, Sallis, Ffrench, \& Owen, 1999). The present thesis focuses on this neglected phase C. Glasgow and colleagues (Glasgow, Vogt, \& Boles, 1999) recognized the problem of lack of diffusion research and developed the RE-AIM model for evaluating public health interventions. This model distinguishes five dimensions that are all assumed to be very important because they interact to determine the public-health or population-based impact of a program or policy: Reach, Efficacy, Adoption, Implementation and Maintenance of an intervention. The last three of these are all aspects of diffusion.

\subsection{Main aims and outline of this thesis}

As shown in section 1.1, the diffusion (adoption, implementation and maintenance) of health promotion guidelines and protocols among healthcare practitioners is an important neglected area of research which warrants thorough research. More knowledge may improve the diffusion of health promotion interventions and thus increase their health impact. This is the issue discussed in the present thesis, which focuses on the diffusion of two protocols, called Minimal Intervention Strategy or MIS, that are designed to help healthcare professionals support their patients to stop smoking. One MIS version is intended for cardiac wards (hospital setting) the other for midwifery practices (primary care office setting). The Integrated Change Model (De Vries, Mesters, Van de Steeg, \& Honing, 2005; De Vries, Mudde, Leijs et al., 2003) served as our comprehensive theoretical framework. This model is described in detail in section 1.7.

The studies described in this thesis had three main aims. The first was to assess the level and quality of use of the MIS for cardiac wards (C-MIS) and the MIS for midwives (V-MIS) in the Netherlands, including identification of the steps of the protocol that are most difficult to apply in daily practice. We also assessed the potential gains if the C-MIS and V-MIS were used in an adequate and consistent manner by all nurses and midwives in the Netherlands. The second aim was to identify and describe factors that are related to successful diffusion of these health promotion protocols among healthcare practitioners. The third overall aim was to assess the applicability of a comprehensive model explaining behavior change, viz. the Integrated Change Model (De Vries et al., 2005; De Vries, Mudde, Leijs et al., 2003) in diffusion research. Since facilitating and impeding factors were expected to differ between the stages of diffusion, two distinct diffusion stages were studied: adoption and implementation.

The next sections in this chapter provide more background information to our studies. Section 1.3 provides information on smoking cessation support by healthcare professionals, and section 1.4 discusses existing Dutch guidelines and protocols on smoking cessation support in healthcare. Section 1.5 provides some theoretical 
information about the diffusion of interventions. Section 1.6 discusses knowledge and knowledge gaps with respect to factors that influence the diffusion of innovations in healthcare organizations, and section 1.7 describes the theoretical framework of this thesis. Section 1.8 provides information on the strategies used to diffuse the MIS among cardiac wards and midwifery practices, and the chapter ends with a brief summarizing conclusion in section 1.9.

Chapters 2 and 3 describe the results of two studies that were conducted on cardiac wards. Chapter 2 presents a cross-sectional study among the heads of all cardiology wards in Dutch hospitals that was performed 4 years after the nationwide introduction of the C-MIS. It describes the various organizational and psychological characteristics that relate to the adoption of the MIS. Chapter 3 presents the results of a cross-sectional study among a sample of cardiac nurses from wards that had adopted the C-MIS. It addresses the level of adherence to the MIS protocol, shows which steps of the protocol were most difficult to perform and identifies the factors that were associated with adherence by cardiac nurses.

Chapters 4, 5 and 6 describe the results of studies in midwifery practices. Chapter 4 describes a cross-sectional study among all Dutch midwifery practices, which was performed 2 years after the nationwide introduction of the V-MIS. It describes organizational and psychological characteristics that are related to the adoption of the VMIS. Chapter 5 presents the results of a cross-sectional study among a sample of midwives from practices that had adopted the V-MIS. It addresses the level of adherence to the V-MIS protocol, shows which steps of the protocol were most difficult to perform and identifies the factors that were most strongly associated with adherence by midwives. Chapter 6 discusses the results of a longitudinal study into the determinants of the initiation of implementation of the V-MIS among midwives. The chapter describes which factors predicted whether practices started to implement the V-MIS.

In chapter 7, a comparison is made between determinants of adoption and implementation in the two different settings, focusing on similarities and differences between the diffusion stages and settings. Finally, chapter 8 discusses and integrates the main results of all studies together, and offers some scientific and practical recommendations.

\subsection{Smoking cessation support in healthcare}

Smoking is responsible for the highest disease burden measured in DALYs (disability adjusted life years), in the Netherlands (RIVM, 2004) as well as in other developed countries (WHO, 2002). Smoking cessation can effectively increase overall survival rates and improve quality of life, or prevent further deterioration of the physical condition of patients suffering from a wide range of diseases, including coronary heart disease and 
COPD (Critchley \& Capewell, 2004; Scanlon et al., 2000; Sherrill, Holberg, Enright, Lebowitz, \& Burrows, 1994). In pregnant women, smoking cessation is important to reduce unfavorable pregnancy outcomes, such as pre-term delivery, low birth weight and fetal death (US Department of Health and Human Services, 2004).

Healthcare professionals frequently have to deal with smokers and smokingrelated health consequences in their daily practice. For example, $49 \%$ of the cardiac patients admitted to a hospital in the Netherlands for coronary problems smoked in the month prior to the hospital admission (Scholte Op Reimer et al., 2006), and about 14\% of Dutch women smoke during pregnancy (Lanting, Van Wouwe, \& Crone, 2005). If health professionals manage to define the appropriate systematic approach, smoking cessation guidance by health professionals can contribute to an effective reduction of smoking rates (Lumley, Oliver, \& Waters, 2004; Rigotti, Munafo, Murphy, \& Stead, 2003) and potentially increase public health. Therefore, national and international evidencebased smoking cessation guidelines for healthcare professionals recommend that they should guide patients and pregnant women in smoking cessation and should use, where possible, interventions based on the Ask, Advice, Assess, Assist and Arrange (5 A's) model (CBO, 2004; Fiore et al., 2000; Raw, McNeill, \& West, 1999; Van Weel et al., 2005). Although several effective interventions based on this model have been developed (e.g. Lumley et al., 2004; Rigotti et al., 2003), a substantial part of the potential public health effects of these interventions may be lost due to non-use or improper use caused by failures in diffusion (Cranney et al., 2001; Glasgow, Lichtenstein, \& Marcus, 2003; Haines \& Donald, 1998).

\subsection{Dutch guidelines and protocols for smoking cessation guidance}

In 2004, the Dutch Institute for Healthcare Improvement (CBO) published the Dutch Guideline for the treatment of tobacco dependence (CBO, 2004). This evidence-based guideline was developed by a team of healthcare professionals and scientists, including representatives of the majority of the Dutch organizations for healthcare specialties, such as cardiology, gynecology, oncology, midwifery, dentistry, psychiatry and general practice. The Dutch expert centre on tobacco control STIVORO and several research institutes were also involved.

The guideline describes a working method to guide smoking cessation that is applicable to every healthcare sector. The essence of this method is independent of the professional's specialty and the health problems for which the patient is being treated. The key elements of the guideline are interventions that increase the motivation to quit smoking. The starting point is that health professionals have to advise each smoking patient at least once to quit smoking, and that, depending on their degree of motivation to quit, patients should receive an intervention that is as intense as reasonably possible 
in the prevailing situation. The working method consists of a stepped care approach to decide on the intensity of the intervention. Although at the time when the guideline was established, a stepped care approach had not been proven to effectively increase abstinence among smokers (Fiore et al., 2000; Riemsma et al., 2003), it was recommended for three reasons: it was believed to increase acceptance and practical applicability, and hence also implementation; it did not prevent the use of more intensive interventions based on clinical views and it was expected to be cost-effective (CBO, 2004).

One example of a method that conforms to this guideline is the so-called Minimal Intervention strategy for Smoking cessation guidance, or MIS. This method had already been diffused to several settings at the time the CBO guideline was developed. Contrary to what might expected from the use of the word "minimal", the MIS is a short supportive intervention that comprises more than merely a brief advice to quit smoking. The MIS comprises a step-wise approach that is based on the Ask, Advice, Assess, Assist and Arrange (five A's) model (Fiore et al., 2000). The various versions of the MIS were developed according to the ABC planning model (De Vries, 1998; De Vries \& Kok, 1996), which was described in section 1.1. The instructions that healthcare professionals receive in the specific MIS versions are adapted to the setting they target. They all consist of the following actions: assess the smoker's profile, advise the smoker to stop smoking, enhance or reinforce their motivation to quit, address barriers and high-risk situations, set a date for quitting, provide educational materials and provide after-care. The MIS is applied at a level that is tailored to the motivational level of each individual patient or client. This enables the health professional to provide the most effective information on smoking cessation in a short period of time.

The first MIS (called H-MIS) was developed for general practitioners and was found to be more effective than usual care in achieving smoking cessation in this setting (one-year point prevalent abstinence $13.4 \%$ vs. 7.3\%) (Pieterse, Seydel, De Vries, Mudde, \& Kok, 2001). The protocol was first implemented in 1994 (Pieterse, 1999). Since then, the MIS protocol has been adapted to several settings. An MIS for midwifery practices (V-MIS) proved to decrease smoking rates among women during pregnancy up to six weeks post-partum (7-day abstinence $21 \%$ vs. $12 \%$ ) (De Vries, Bakker, DolanMullen, \& Van Breukelen, in press). An MIS for cardiac wards (C-MIS) proved to increase smoking cessation rates among cardiac inpatients three months after hospital discharge (point prevalent abstinence 52\% vs. 39\%) (Bolman, De Vries, \& Van Breukelen, 2002a). In view of the results of a process evaluation about the level of use and conflicting results of analysis strategies revealing ineffective long-term effects one year after hospital discharge (using intention-to-treat analysis), the recommendation was made to improve the after-care component (Bolman, De Vries, \& Van Breukelen, 2002a, 2002b). Two 
studies failed to find support for the effectiveness of the C-MIS in outpatient settings (Berkel, 2000; Wiggers et al., 2005), although the study by Wiggers, in which the C-MIS was added to the provision of nicotine replacement therapy, did result in a clinically interesting $5 \%$ increase in 7-day abstinence after one year. Recently, a more extended version of the MIS has been developed for the outpatient treatment of patients with lung problems (L-MIS). This version has not yet been formally tested for its efficacy or effectiveness compared to no intervention, but a randomized controlled trial that compared the effects of the medium-intensity L-MIS with the effects of a high-intensity smoking cessation program showed that the L-MIS resulted in a 12-month pointprevalent smoking cessation rate of $12 \%$ and a continuous abstinence rate of $9 \%$ (Christenhusz, 2006).

\subsection{Theoretical considerations on the diffusion of interventions}

Implementation strategies that target the main determinants of successful diffusion of smoking cessation interventions among healthcare providers and take the main barriers into account are assumed to improve the diffusion of these interventions and so increase their health impact (Grimshaw et al., 2004). In the diffusion process of interventions, three stages can be distinguished. The first is the adoption stage; a decision-maker learns of the existence of an intervention (knowledge) and then forms a favorable or unfavorable attitude towards this intervention (persuasion). Together with other factors, this eventually leads to the decision to either adopt or reject the intervention (decision). The second stage is the implementation stage. After an intervention has been adopted, the next step is to successfully implement it, which means that it is put into use as intended. Following implementation, there is one final stage, called maintenance or confirmation, in which the future use of the intervention is secured (Rogers, 1995).

Diffusion of an intervention in an organization can be seen as changing the behavior of a large group of individuals. This issue has been studied by various disciplines, ranging from marketing to psychology, using various theories about factors that have the potential to influence successful diffusion of interventions. These theories can be classified into three groups, based on the type of characteristics they focus on. First, there are theories that focus on organizational factors, such as the contingency model of strategic decision-making (Koopman \& Pool, 1991, 1994) and theories on the characteristics of innovative organizations (Damanpour, 1991). Studies based on organizational theories have identified a variety of factors that influence diffusion, such as organization size (Castle, 2001; Moch \& Morse, 1977), level of centralization (Damanpour, 1991; Harting et al., 2005) and level of formalization (Zaltman, Duncan, \& Holbek, 1973). Second, there are theories that focus on characteristics of individuals who need to adopt and use an intervention. This group includes several social-cognitive 
theories, like the theory of planned behavior (Ajzen, 1991, 1995), the Precede/Proceed model for planning changes (Green \& Kreuter, 1999), the Transtheoretical model (Prochaska \& DiClemente, 1983), the social cognitive theory (Bandura, 1986), the social influence theory (Mittman, Tonesk, \& Jacobson, 1992) and the ASE model (De Vries, Dijkstra, \& Kuhlman, 1988; De Vries \& Mudde, 1998). These theories assume that behavior is influenced by an individual's intention to engage in a particular behavior, which is in turn influenced by cognitive perceptions and the perceived influence of the social environment. Studies applying these theories have found significant relations between the diffusion of various health promotion interventions and factors like attitude towards the intervention (Fahrenwald \& Walker, 2003; Mesters \& Meertens, 1999; Haagen et al., 2005; Michie, Hendy, Smith, \& Adshead, 2004), perceived social influence (Paulussen, Kok, \& Schaalma, 1994; Wilhelm, 2002) and self-efficacy towards using the intervention (Wilhelm, 2002; Fahrenwald \& Walker, 2003; Haagen et al., 2005). A third type of theory is the diffusion theory (Rogers, 1995), which focuses specifically on the characteristics of the intervention itself. It states that successful diffusion is strongly related to five intervention characteristics, namely the relative advantage, compatibility, observability, trialability and complexity of an intervention. As intervention characteristics are assumed to affect diffusion through an individual's perception of the intervention (Rogers, 1995), some studies based on social-cognitive theories have included these factors as part of the attitude concept (e.g. Bolman et al., 2002; Paulussen et al., 1994; Bakker, De Vries, Mullen, \& Kok, 2005).

\subsection{Factors that influence diffusion of innovations in healthcare}

The previous section showed that studies based on a range of theories have identified a wide variety of factors that may influence the diffusion of interventions. Although most studies on diffusion have been performed outside the field of public health, studies on the determinants of the diffusion of innovations in healthcare organizations have shown that the organizational, individual and intervention characteristics also affect the diffusion of interventions in such organizations. Fleuren and colleagues (Fleuren, Wiefferink, \& Paulussen, 2004) performed a systematic literature search on characteristics affecting the diffusion of interventions among healthcare organizations. Based on the 57 studies they retrieved, they listed 50 determinants that potentially affect the innovation process in such organizations, which included the organizational, individual and intervention characteristics mentioned above. For example, high staff turnover and insufficient staff capacity were found to impede innovation, as did lack of support and low self-efficacy, incompatible and unappealing innovations and lack of available time (Fleuren et al., 2004). Some diffusion research has also been done with respect to smoking cessation support by healthcare practitioners. These studies showed, for example, that the 
perceived advantages and simplicity of the intervention were important facilitating factors for the use of the MIS by cardiac nurses (Bolman et al., 2002) and that perceived advantages and perceived social support increased the chances that midwives wanted to provide their clients with smoking cessation advice (Bakker et al., 2005) .

Unfortunately, however, these previous studies failed to assess the relative importance of the various factors within a more comprehensive framework, and did not study all factors together. Additionally, very few studies distinguished and compared the three diffusion stages. Making a distinction between stages is important, since the studies that did make this distinction showed different influences of factors in each diffusion stage (Zaltman et al., 1973; Cooke, Mattick, \& Campbell, 1999; Orlandi, 1987). For example, centralization and formalization have been found to inhibit early diffusion stages like adoption, but have been found to promote later stages like implementation (Zaltman et al., 1973), and self-efficacy has been found to be mainly associated with maintenance and only to a minor extent with adoption (Cooke, 2000).

The fact that the specific and detailed information on barriers to change in a particular setting is often limited seems to be a very important reason for the fact that up till now no solid basis was found for recommending a particular strategy for implementing innovations (Hulscher, Wensing, Van der Weijden, Grol, 2003; Grimshaw et al., 2004).

\subsection{Research framework}

To help bridge these gaps in available knowledge, the main theoretical issue addressed in the present thesis is the assessment of the most important determinants of the adoption and implementation of the MIS interventions in healthcare and of the extent to which the relative importance of determinants differs between adoption and implementation. Apart from this theoretical issue, the general aims of the studies described in this thesis were defined in section 1.2.

As the generalizability of study findings to other settings is often difficult to judge (Foy, Eccles, \& Grimshaw, 2001; Glasgow et al., 2003), we studied two different types of healthcare settings. To identify the potential organizational, individual and intervention characteristics that could determine adoption and implementation, a comprehensive theoretical framework was used, known as the Integrated Change Model for exploring motivational and behavioral change (I-Change model) (De Vries, Mesters, Van de Steeg, \& Honing, 2005; De Vries, Mudde, Leijs et al., 2003; De Vries, Mesters, Van 't Riet, Willems, \& Reubsaet, 2006), which was supplemented with factors derived from the contingency model of strategic decision-making (Koopman \& Pool, 1991, 1994). Since the I-Change model had so far primarily been used for research on individual health promotion behaviors, the studies described in this thesis tested the applicability of this model in diffusion research. 
The Integrated Change Model was derived from the attitude-social influence-selfefficacy (ASE) model (De Vries, Dijkstra, \& Kuhlman, 1988; De Vries \& Mudde, 1998). It can be regarded as an integration of the ideas contained in various social-cognitive models (Ajzen, 1995; Bandura, 1986; Gollwitzer \& Brandstätter, 1997; Janz \& Becker, 1984; Prochaska \& DiClemente, 1983; Weinstein, 1988) with the diffusion theory (Rogers, 1995). This combination improves the model's ability to predict diffusion (Parcel et al., 1995). The model also includes predisposing factors, like organizational factors and a person's demographic characteristics. The model is presented in Figure 1.

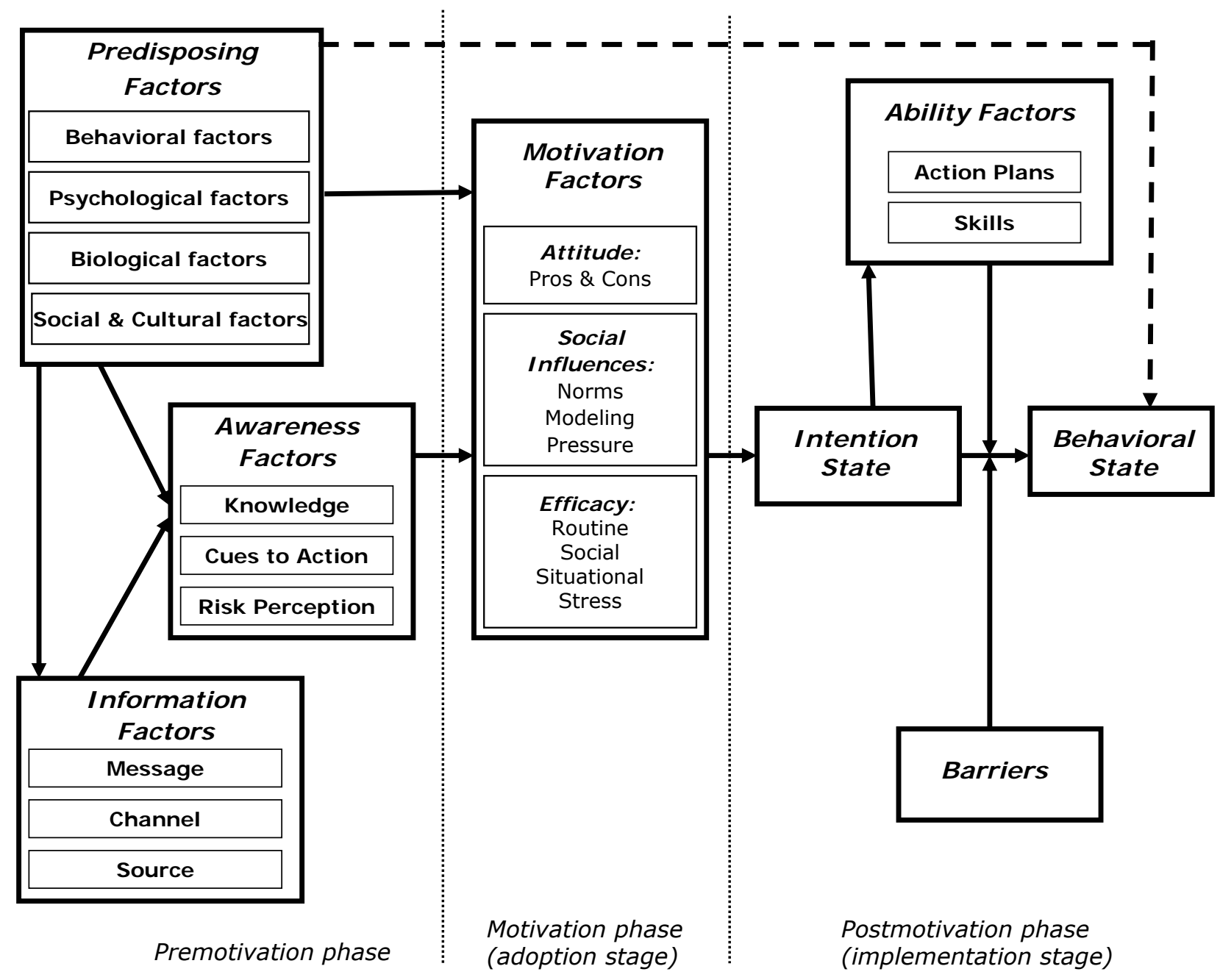

Figure 2. The Integrated Change Model (De Vries et al., 2005, 2006; De Vries, Mudde, Leijs et al., 2003)

The I-Change Model states that behavior is determined by several factors, and that three different motivation phases are relevant: the pre-motivation phase, the motivation phase and the post-motivation phase. The pre-motivation phase is characterized by the fact that persons may not be aware that there is a specific problem or issue relevant to them, in our case the existence of a new protocol. The motivation 
phase is the phase in which the intention is formed to engage in a behavior, in this case the decision to use a protocol. The post-motivation phase involves the transition from a general goal intention to the actual behavior, in this case the use of the protocol. As adoption of an intervention can be regarded as the positive intention to use it, we considered the adoption stage to be comparable to the motivation phase. As implementation results in the use of a protocol that one had previously decided on using, we considered the implementation stage to be analogous to the post-motivation phase.

According to the I-Change Model, behavior is most likely to be determined by intentions, ability factors and barriers. A person's abilities increase the likelihood that intentions are transferred into actions, while barriers can reduce this likelihood. Abilities include matters like being able to prepare and carry out specific plans to achieve the intended behavior (Gollwitzer \& Brandstätter, 1997), as well having the actual behavioral skills necessary to perform the behavior. Examples of barriers include lack of time or money.

A positive or negative intention to engage in a particular behavior is determined by three types of motivation factors (De Vries, Mesters, Van de Steeg, \& Honing, 2004). The first factor is an individual's attitude towards a new behavior, which is assumed to be the result of weighing the perceived pros (advantages) and cons (disadvantages) of its use. An individual's perception of the characteristics of the intervention (Rogers, 1995) are considered to be part of the pros and cons. The second is the social influence encountered, which consists of the perception of others carrying out this type of behavior (social modeling), the norms that people have with respect to these behaviors (social norms) and the support or pressure that someone encounters from others towards engaging in a particular type of behavior (social support). The third type is that of selfefficacy expectations. Self-efficacy refers to a person's perception of their ability to carry out the intended behavior. Recent studies showed that different types of self-efficacy may exist, such as social, stress, skills and routine self-efficacy (De Vries, Mudde, Kremers et al., 2003; Dijkstra \& De Vries, 2000; Holm, Kremers, \& de Vries, 2003).

The I-Change Model assumes that awareness factors influence motivation factors. These factors are knowledge, risk perceptions and cues to action, the latter derived from the Health Belief Model (Janz \& Becker, 1984). The awareness factors also reflect the input from the Precaution Adoption Model (Weinstein, 1988), which indicates that many people may not yet be aware of a health risk in general, or of their own level of risk. Many people have misconceptions about their actual health behavior; they may believe that they are actually already performing adequately, and may therefore not be aware of their personal risk (Bogers, Brug, van Assema, \& Dagnelie, 2004; Lechner \& Brug, 1997).

Factors that determine the pre-motivational, motivational and post-motivational phases are information factors (the quality of messages, channels and sources used) and 
predisposing factors (e.g. biological factors like gender and genetic predisposition, and social and cultural factors like cigarette prices and policies) (De Vries, Mudde, Leijs et al., 2003). Whereas the assumption is that the influence of predisposing factors is largely exerted through awareness factors, direct influences on behavior are also possible. For instance, the impact of past behavior has been found to exert direct influences on behavior that are not mediated by cognitions (Lechner, De Nooijer, \& De Vries, 2004; Norman \& Conner, 2006).

Predisposing factors are indicated by the I-Change Model as playing a role in behavior change, but the model does not extensively define a large set of important predisposing factors, partly because they may differ between different types of behavior and context. To define the predisposing factors in our situation in more detail, we used a research model called the contingency model of strategic decision-making. This model originated in an organizational psychology perspective and has been developed by Koopman and Pool (Koopman \& Pool, 1991, 1994). The applicability of this model to the field of health promotion has been discussed by Willemsen and colleagues (Willemsen, Meijer, \& Jannink, 1999). The basic assumption of the model is that circumstances strongly determine the decision-making process in an organization. The model defines three major groups of characteristics influencing the decision-making process: the content of decision-making, its context, and the decision-making style that is dominant in the organization. With respect to the content, Pool and Koopman (Pool \& Koopman, 1992) distinguish the novelty of a problem, its ambiguity, its importance, its size or scope and the degree to which a decision is strategically embedded. In our research, the content of decision-making was the same for all research subjects, i.e. using the MIS, so no specific information on this aspect was collected. With respect to the context of decision-making, the model distinguishes between characteristics of the environment (e.g. university or general hospital, patient characteristics), of the organization (e.g. size, workload) and of the decision-maker (e.g. age, gender, innovativeness or smoking behavior). The third group of characteristics, relating to the decision-making style, includes the level of centralization, the level of formalization, the way information is gathered, and the handling of confrontations (Koopman \& Pool, 1991, 1994).

Centralization is the extent to which decision-making is restricted to the top management or left to individuals at lower hierarchical levels. Formalization is the extent to which decisions at the workplace are regulated by an established set of procedures or are based on individuals' own solutions. With respect to the way information is gathered, distinctions can be made for example between internal and external information sources or the overall amount of information consulted. The handling of confrontation refers to both the extent to which confrontation and conflict exists in the decision-making process and the way such conflicts are dealt with, e.g. compromise, clash, postpone. These three 
groups of characteristics generally fit into the "social and cultural factors" box in the IChange model, except for the characteristics of the decision-maker (e.g. age, gender and smoking behavior). These are incorporated with the behavioral and biological factors.

\subsection{Diffusion strategies of the MIS}

The national diffusion program for the C-MIS was executed by STIVORO between 2000 and 2004 and for the V-MIS between 2002 and 2004. The aims of the C-MIS diffusion program were that at the end of the period, the C-MIS would be used on $66 \%$ of the cardiac inpatient wards and that $33 \%$ of all nurses on cardiac inpatient wards would use the C-MIS. The aim of the V-MIS diffusion program was that at the end of the period, the V-MIS would be used by $50 \%$ of the midwives. This section describes the strategies that were used to diffuse the C-MIS and V-MIS among cardiac nurses and midwives respectively. The descriptions of the strategies are derived from the Dissemination and Implementation Plans of the C-MIS (Kans, 2004) and the V-MIS (Roovers, 2005). To put these strategies into perspective, the organization of care in each of the two settings is briefly described below, before the actual diffusion strategies are described.

\subsubsection{Cardiac nursing care}

In total, Dutch hospitals accommodated 121 cardiac inpatient wards in 2003. On average, these wards employed 24 cardiovascular nurses and four cardiologists, they had 20 beds and admitted about 90 patients per month (STIVORO database, 2004). About $30 \%$ of the cardiac nurses were members of the Dutch organization for cardiovascular nurses (NVHVV, 2003). Cardiology wards usually have a high level of autonomy in terms of decision-making. Both nurses and cardiologists play a major role in decisions on protocols for treatment and changes at the ward. A key person in the ward network is the ward head. In Dutch cardiology wards, this person usually works as a nurse on the ward. Ward heads and cardiologists share responsibility for the working procedures at the ward. Cardiologists are responsible for the medical aspects of the work, while ward heads are primarily responsible for the nursing part of the work. The ward head has the decisional power and status to decide on the adoption of interventions like the C-MIS, because these interventions are mainly performed by the nursing staff. Apart from adopting new interventions and protocols, the ward head is also the person who maintains contact with all ward employees and with other disciplines related to the ward, such as physicians, physiotherapists and dieticians. Today, virtually all hospital wards in the Netherlands use a patient-centered nursing system as opposed to the task-centered system that used to be popular before. This means that nurses have their "own" patients as much as possible, for whom they perform all necessary care tasks. Only when shifts 
change are patients transferred from one nurse to another. This transfer usually takes place by examining the report of the previous shift together with the patient.

\subsubsection{Diffusion of the MIS for cardiac wards (C-MIS) in the Netherlands}

After the C-MIS had been studied for its effectiveness, its diffusion was managed by the Dutch expert centre on tobacco control, called STIVORO for a smoke free future. The implementation strategy was developed with the support of a working group in which several disciplines were represented: a researcher, a cardiologist, a general practitioner, a cardiac nurse, a representative of the Dutch Heart Foundation, a representative of the Ministry of Health, a representative of the Netherlands Organization for Health Research and Development and STIVORO staff. It was decided to use a direct and personal approach for implementation (i.e. an appointed project leader for implementation of the MIS contacted every ward personally and if they were interested, arranged a face-to-face meeting with an implementation trainer). This choice was based on several considerations. One was that the cardiologists and especially cardiac nurses were already highly committed to the topic, and cardiology wards usually have a high level of decisionmaking autonomy. Furthermore, the relatively small number of 121 cardiac inpatient wards in the Netherlands made it possible to adapt the implementation strategy to the characteristics of the individual hospitals in a personalized approach. Some facilitating factors for the use of the MIS by nurses had been identified in a pilot study of the C-MIS and in the process evaluation of an effectiveness study of the C-MIS. The most important facilitating factors for the use of the protocol included perceived advantages and simplicity of the protocol, which can be promoted by proper training (Bolman, 2001; Bolman, De Vries, \& Mesters, 2002). Research showed that ward representatives needed to be trained on the conditions that had to be met on the ward before the C-MIS could be introduced, on the use of smoking cessation guidance as a part of the ward policy and on ways to include the MIS in the initiation period for new colleagues. Nurses had to be trained in the mechanism of the intervention, in techniques to start a conversation about smoking and in ways to handle difficult situations (Bolman, 2001; Bolman, De Vries, \& Mesters, 2002). Furthermore, previous research on the diffusion of tobacco prevention programs had shown that diffusion strategies should focus on the attitudes of the decision-makers and that those responsible for the administration and implementation of programs should be included in efforts to influence the decision-making process (Parcel et al., 1995).

The above factors were taken into account when designing the implementation strategies. In addition, principles from Rogers' diffusion theory were used, such as the fact that the main elements of the diffusion process are the intervention, communication channels, time and the social system (Rogers, 1995). First, all employees on cardiac 
wards were informed about the existence of the C-MIS by means of a mailing, which was sent to all cardiologists, cardiology students, all cardiac nurses that were members of the NVHVV and the ward heads of all cardiac wards. The information, which stressed the importance of the MIS, consisted of information leaflets about the C-MIS, one for cardiologists and one for nurses, and a letter that was signed by the managing directors of the Dutch Heart Foundation and STIVORO and by the presidents of the Dutch organization for cardiologists (NVVC) and the NVHVV, who were considered to be opinion leaders in the field of smoking and cardiovascular disease. The information leaflets contained information on the nature, intentions and application of the C-MIS. About two weeks later, a second mailing was sent to all cardiac wards, with the request to supply STIVORO with one or more contact persons who could be approached for C-MIS related subjects. If wards did not respond, they were contacted by phone.

Subsequently, a C-MIS introduction package including all educational materials (e.g. implementation manual, skills manual, instruction video and flow charts) was sent to all wards, accompanied by a letter offering an implementation training by STIVORO. Wherever possible, the package was addressed directly to the contact person. The implementation training was a one day training in which contact persons were taught a stepwise strategy for introducing the C-MIS on their ward, based on the team quality improvement sequence (TQIS) (Ovretveit, 1999). The main aim of the implementation training was to increase the contact persons' self-efficacy towards implementing the CMIS on the ward (DEFACTO, 2002), for instance by means of role plays. The implementation training was supported by an implementation manual which included a comprehensive overview of the stepwise strategy for introducing the C-MIS on the ward. After the implementation training, contact persons were assigned a trainer-coach, who could be consulted in case of problems or questions. The trainer-coaches had two meetings with each contact person. The first was an advisory interview at the beginning of the implementation process, during which the main expected barriers were discussed. About six months after the introduction of the C-MIS on the ward, the implementation process was evaluated with the trainer-coach in a second meeting, and the barriers that had been encountered were discussed.

As soon as the contact person indicated that the ward had decided to use the CMIS, an in-house skills training was offered by STIVORO for all nurses. The training took four hours and focused on providing smoking cessation guidance according to the C-MIS and on ways to carry out the steps of the protocol. It incorporated interviewing techniques (trained by means of role plays), information on understanding smoking behavior, addiction and quitting, and information on the principles and effectiveness of the C-MIS. The skills training was supplemented with a skills manual and a video that illustrated the steps of the C-MIS in detail. Furthermore, wards were provided with 
intervention cards that functioned both as a flow chart and as a recording system for the steps of the C-MIS.

After the training, several contact days were organized for C-MIS users, allowing information on C-MIS related topics to be presented and enabling nurses to share experiences. During the implementation period, several advertisements and articles were published in professional journals, presentations were given at conferences and other events, and a biannual newsletter on the C-MIS was sent to the contact persons and all ward heads, cardiologists and cardiology students. The implementation activities that were performed on each cardiac ward were recorded by STIVORO, the Dutch expert centre on tobacco control, in an electronic implementation recording system, together with accurate information on the MIS materials that were ordered by the wards.

All implementation activities were funded by ZonMw - the Netherlands Organization for Health Research and Development.

\subsubsection{Midwifery care}

In the Netherlands, all regular pregnancies are assisted by midwives and general practitioners. The greater part of midwifery care is taken care of by midwives; only $6 \%$ of the general practitioners provide pregnancy care (Wiegers \& Van Wieren, 2005). Primary care midwives, a category covering $83 \%$ of the 1955 midwives in the Netherlands (Nivel, 2004), assist about $75 \%$ of all pregnant women (TNO, 2000). Only when complications or exceptional risks are identified, are pregnant women referred to an obstetrician.

Midwives are organized in 450 practices (Nivel, 2004). Nine percent of the midwives work in one-person practices, $18 \%$ work in two-person practices and $73 \%$ work in group practices with at least two other midwives. About half of the group practices have practice assistants who can take over some tasks, such as administrative tasks, answering the phone and preparing consultations (Nivel, 2004). Group practices are based on the principle of equality of the members and do not have a specific leader. All midwives receive equal training and have the same qualifications. Within practices, midwives work on the basis of collective agreements and written guidelines, which is necessary since clients in most practices may circulate among midwives.

Midwifery care is coordinated by the Royal Dutch Organization for Midwives (KNOV), a professional association of which $91 \%$ of the midwives are members. The KNOV has 60 regional subdivisions called "circles". The task of these circles is to implement and support the general KNOV policies at regional level and to stimulate and improve cooperation between midwives within a region. Within a circle, midwives working in the same region make agreements on policy and cooperation. 


\subsubsection{Diffusion of the MIS for midwives (V-MIS)}

The diffusion of the V-MIS was managed by STIVORO. The KNOV was approached by STIVORO and was found to be willing to support the implementation of the V-MIS where possible. Subsequently, a working group was formed and involved in the development of the implementation strategy. Several disciplines were represented, viz. a researcher, a representative of the KNOV, a representative of the Ministry of Health, a representative of the Netherlands Organization for Health Research and Development and STIVORO staff. The group examined the characteristics of the target group for providing the MIS, i.e. midwives, obtaining helpful information from a pilot study of the V-MIS (Bakker, De Vries, Mullen, \& Kok, 2005). Important focal points were workload, client relation, selfefficacy and practice organization.

To inform all midwives of the existence of the V-MIS, a leaflet and a letter signed by STIVORO and KNOV, who were considered to be opinion leaders in the field of smoking and pregnancy, were sent to all midwifery practices and regional circles. Besides contact information, the leaflet contained information on the goal and content of the VMIS and on the available supportive educational materials for pregnant women and midwives. The letter offered information about the content of the V-MIS and about a skills training that was offered by STIVORO in cooperation with KNOV. The free-of-charge skills training program was offered to primary care midwives within their own region, and took three hours. Part of the training session involved teaching interviewing techniques by means of role plays. The skills training program had been certified by KNOV. The skills training was supplemented with a skills manual, which extensively discussed the steps of the MIS. Furthermore, midwifery practices were provided with intervention cards, which functioned both as flow charts and as a recording system for the steps of the V-MIS, and with educational materials for pregnant smokers.

The start of the nationwide MIS diffusion was publicized by means of a press release and a public presentation of the V-MIS educational materials by the chairman of KNOV and the managing director of STIVORO. Twice a year, a joint newsletter on the VMIS and a project aimed at reducing smoking in the presence of small children was sent to midwives, obstetricians, maternity nurses and other relevant parties in the field, including infant welfare centers. When midwives decided to work with the MIS, they were provided with additional educational materials for pregnant women, such as a waitingroom poster, educational magazines including partner guides, educational videos and relapse prevention booklets for pregnant women. The implementation activities that were performed in each midwifery practice were recorded by STIVORO in an electronic implementation recording system, together with detailed information on the V-MIS materials that were ordered by the practices. During the implementation period, several advertisements and articles were published in professional journals, presentations were 
given at conferences and other events and biannual newsletters on the V-MIS were sent to all midwifery practices.

All implementation activities were funded by ZonMw - the Netherlands Organization for Health Research and Development.

\subsection{Conclusion}

Research on the diffusion of health promotion programs in the healthcare system has been scarce. Even scarcer has been research analyzing the factors determining adoption and implementation, and whether these may differ between or settings. These items are the main topics of the present thesis and are discussed in the remaining chapters. $A$ range of theories was used to identify a wide variety of factors that may influence the diffusion of interventions. To identify potential determinants of adoption and implementation, we used a comprehensive theoretical model, the Integrated Change Model, as our theoretical framework. As smoking results the highest disease burden in terms of DALYs and smoking cessation protocols have proved to be able to effectively reduce smoking rates, the present thesis focuses on the diffusion of protocols for smoking cessation support by health professionals (MIS). Two settings were studied, i.e. cardiac wards and midwifery practices. 



\section{CHAPTER 2}

Determinants of Adoption of Cognitive Behavioral Interventions in a Hospital Setting: Example of a Minimal-Contact Smoking Cessation Intervention for Cardiology Wards 


\section{Abstract}

A cross-sectional survey by means of questionnaires was conducted among the heads of all 121 cardiology wards in Dutch hospitals, of whom 77 (64\%) responded. The aim of this study was to identify the psychological and organizational determinants of adopting a smoking cessation intervention in cardiology wards, using the Integrated Change Model as the theoretical basis. The results show that adopters had a significantly lower level of formalization and more nurses working on the ward. Adopters also had a significantly more positive attitude towards working with the intervention, perceived more positive social influences from their direct environment and had higher selfefficacy. Psychological determinants explained adoption to a greater extent than organizational determinants did. In conclusion, it is very important to take psychological factors into account when studying the adoption of preventive innovations and developing diffusion strategies and programs for dissemination of preventive innovations in a hospital setting should primarily focus on psychological characteristics like social influence and self-efficacy.

\section{Slightly different version published as:}

Segaar, D., Bolman, C., Willemsen, M.C., De Vries, H. (2006) Determinants of Adoption of Cognitive Behavioral Interventions in a Hospital Setting: Example of a MinimalContact Smoking Cessation Intervention for Cardiology Wards. Patient Education and Counseling 61(2), 262-271 


\subsection{I ntroduction}

Cigarette smoking is a major cause of cardiovascular disease (Burns, 2003; Tsiara, Elisaf, Mikhailidis, 2003) and smoking cessation reduces the mortality risk of patients with coronary heart disease (Critchley \& Capewell, 2004). During hospitalization, patients are more receptive to information on smoking cessation, since they are more aware of their personal vulnerability (Emmons \& Goldstein, 1992; Orleans, Rotberg, Quade, \& Lees, 1990). For these reasons, the opportunity to help people stop smoking during hospital admission should be seized upon.

Much research has been done into effective smoking cessation interventions for hospitalized patients (France, Glasgow, \& Marcus, 2001; Rigotti, Munafo, Murphy, \& Stead, 2003). Despite their effectiveness, the adoption of these interventions in hospital practice often fails (France et al., 2001; Haines \& Donald, 1998). Adoption has been studied by researchers from several disciplines, ranging from business marketing to psychology. These studies identified a wide variety of factors that might influence adoption, such as the size of the organization (Castle, 2001; Moch \& Morse, 1977), group membership (Castle, 2001), and innovation characteristics (Rogers, 1995). The problem with such studies has been that the results are difficult to extrapolate to other settings (Foy, Eccles, \& Grimshaw, 2001; Glasgow, Lichtenstein, \& Marcus, 2003). Hence, research is needed to identify reasons for adoption in specific settings and specific populations.

Our study aimed to identify psychological and organizational determinants of the adoption of an intervention for smoking cessation guidance at cardiology wards. As far as we know, no other studies have been published on the adoption of preventive innovations at cardiology wards. Although we found some studies on the adoption of preventive interventions in a hospital setting (Fiset \& Grembowski, 1997; Sinclair, Maxfield, Marks, Thompson, \& Gershon, 2002), these did not address psychological determinants.

The present study focused on a Minimal Intervention Strategy for Smoking cessation for Cardiac inpatients (C-MIS), which has been disseminated to Dutch cardiology wards nationwide since 1999. The C-MIS is a stepwise strategy in which cardiologists and nurses cooperate in assisting smokers to quit smoking, and was recently tested for its effectiveness (Bolman, De Vries, \& Van Breukelen, 2002a; 2002b). STIVORO, the Dutch expert centre on tobacco control, took care of the dissemination. Over a period of four years, STIVORO distributed information on the C-MIS through various channels, such as direct mailing, professional journals, and conferences. Training courses on how to introduce the C-MIS at the ward and how to use it were offered to cardiology nurses, and supporting materials like manuals and information brochures for patients were widely distributed. 
To define the determinants of adoption of the C-MIS, we used the Integrated Change Model (I-Change Model) (De Vries, Mesters, Van de Steeg, \& Honing, 2005; De Vries, Mudde, Leijs et al., 2003; De Vries, Mesters, Van 't Riet, Willems, \& Reubsaet, 2006). This model integrates concepts of various cognitive models, such as the Social Cognitive Theory (Bandura, 1986), the Theory of Planned Behavior (Ajzen, 1991, 1995), the Transtheoretical Model (Prochaska \& DiClemente, 1983), and the Precaution Adoption Model (Weinstein, 1988). It also includes predisposing factors, like organizational factors and individual characteristics.

The I-Change Model states that behavior can be predicted by an individual's intention, which is determined by three types of motivational factors (De Vries, et al., 2005). The first is an individual's attitude towards a new behavior, which is assumed to be the result of weighing the perceived pros (advantages) and cons (disadvantages) of its use. The second is the social influence encountered, i.e., norms, modeling, and pressure. The third type is that of self-efficacy expectations. The Attitude, Social Influence, and Self-Efficacy concepts have already been used to explain the adoption of various types of health behavior, like the adoption of classroom-based AIDS education (Paulussen, Kok, \& Schaalma, 1994), the adoption of hormone replacement therapy for menopausal complaints (Wilhelm, 2002), and the adoption of physical activity behavior by mothers in the Women, Infants, and Children (WIC) program (Fahrenwald \& Walker, 2003). The I-Change Model assumes that these motivational factors are determined by various distal factors such as awareness factors (i.e., knowledge, personal relevance, and risk perceptions), information factors (the quality of messages, channels, and sources used), and predisposing factors (e.g. behavioral factors, biological factors, and social and cultural factors) (De Vries, Mudde, Leijs et al., 2003). Information factors were not included in our study since the possible sources from which wards could gather information on the C-MIS, with their accompanying messages and channels, were the same for all wards.

The predisposing factors were defined on the basis of the theory about decisionmaking processes from the organizational psychology perspective developed by Koopman and Pool (Koopman \& Pool, 1991, 1994). The applicability of this model in implementation research has been described by Willemsen et al. (Willemsen, Meijer, \& Jannink, 1999). Koopman and Pool defined three major groups of characteristics influencing the decision-making process: the content and context of decision-making, and the decision-making style of the organization. Since in our research, the decisionmaking content was the same for all wards, namely introducing the C-MIS, it was not included in our study. The context consisted of characteristics of the environment (e.g., being a ward of a university hospital), the organization (e.g., ward size), and the decision-maker (e.g., age, being a smoker). Characteristics of the decision-maker belong 
to the behavioral and biological factors in the I-Change Model, while the organizational and environmental context and the decision-making style belong to the social and cultural factors in this model. Decision-making style may vary in terms of the level of centralization, the level of formalization, the handling of confrontation, and the way information is gathered. High levels of centralization and formalization are assumed to inhibit adoption (Rogers, 1995).

The results of the present study can be used to develop research-based implementation strategies to further increase and facilitate adoption of interventions in hospital settings. The Integrated Change Model that we used to study adoption is presented in Figure 1.

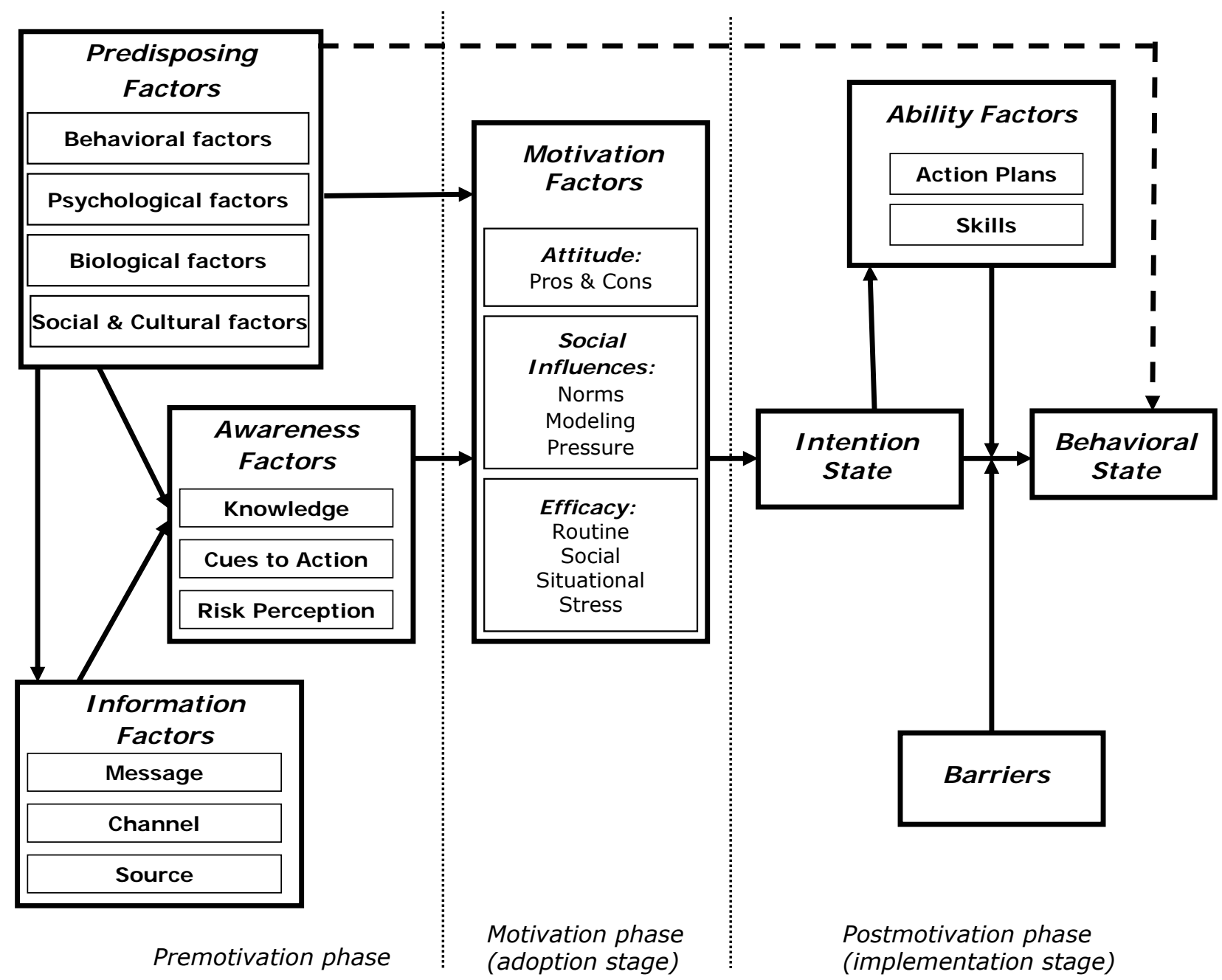

Figure 1. The Integrated Change Model (De Vries et al., 2005, 2006; De Vries, Mudde, Leijs et al., 2003) to study adoption of the C-MIS. 


\subsection{Methods}

\subsubsection{Study design and procedure}

A cross-sectional study was conducted among all heads of cardiology wards in Dutch hospitals ( $\mathrm{N}=121)$, four years after the start of the nationwide dissemination of the CMIS. Cardiology wards usually have a high level of autonomy in terms of decisionmaking, and both nurses and cardiologists play a major role in deciding on changes at the ward. A key person in the ward network is the head of the ward. In Dutch cardiology wards this person most of the time works as a nurse on the ward. Nursing ward heads and cardiologists share the responsibility for the working procedures on the ward. Cardiologists are responsible for the medical parts of the job. Ward heads have primary responsibility for the nursing part of the job. The C-MIS is mainly performed by the nursing staff, therefore the ward head has the decisional power and status to decide on adoption of the C-MIS. Apart from giving permission for the use of new interventions and protocols, the ward head also maintains contacts with workers on the ward and with other disciplines related to the ward, such as physicians, physiotherapists, and dieticians. For these reasons, ward heads were the subject of our study. Written questionnaires were sent to all heads of cardiology wards in the Netherlands. A cover letter and a postage-paid return envelope were enclosed. The letter provided information about the purpose of the study and ensured confidentiality. The Dutch Federation of Hospitals (NVZ) provided hospital addresses. Ward heads were invited to fill in the questionnaire in their role as "representative of the ward". Respondents had a chance to win a $€ 25$ value coupon if they returned the questionnaire. Postal reminders were sent to non-responders two weeks after the first mailing.

\subsubsection{Questionnaire}

The questionnaire was based on existing questionnaires or relevant scales, and information from literature reviews and earlier work (Bakker, 2001; Steckler, Goodman, McLeroy, Davis, Koch, 1992; Bolman, De Vries, Mesters, 2002; Mesters, \& Meertens, 1999). It started with a brief introduction explaining the C-MIS.

\section{Outcome variable}

To be able to distinguish between adopters and non-adopters, the following questions were asked. First, ward heads were asked if they were already using the C-MIS. If not, they were asked two additional questions: (1) if they had made a conscious decision about using the C-MIS at all, and (2) if they intended to use the C-MIS in the future and if so when (i.e., within the next month, within the next six months, within the next year, or within the next five years). Adopters were those respondents who were either using the C-MIS or had decided to do so in the near future, defined as six months from the 
moment they filled in the questionnaire.

\section{Predisposing factors}

For detailed information on the questions, see Table 1.

Behavioral factors assessed were the smoking behavior of the ward head, the number of years that the heads had been working in their current profession, the number of years they had worked at their current ward, and their innovativeness (rated on a 5 -point scale ranging from $-2=$ very negative attitude towards changes to $+2=$ very positive attitude towards changes). Biological factors assesses were the age and gender of the ward head. Social and cultural factors included context characteristics of the organization and its environment and the decision-making style of the organization. Measurements of context characteristics at the environment level included the type of hospital (general/university) and the innovativeness of the hospital board (rated on a 5point scale ranging from $-2=$ very negative attitude towards changes to $+2=$ very positive attitude towards changes). Measurements of context characteristics at the organizational level included ward size (number of nurses and number of beds), nursing system (patient-oriented or task-oriented), innovativeness of the ward team, and availability of resources in terms of both money and employees $(-2=$ highly insufficient, $+2=$ highly sufficient). In addition, the following dimensions of the decision-making style were measured: centralization (rated on a 5 -point scale ranging from $0=$ mode of operation entirely decided by ward to $4=$ decisions on mode of operation completely centralized at hospital level); formalization of decision-making on the ward (rated on a 5-point scale ranging from $0=$ always own solutions, $4=$ always set procedures); the amount of information gathered before a decision was made (rated on a 5-point scale ranging from $0=$ no information at all to $4=$ all possible information); the proportion of external information sources versus internal information sources used to make a decision (rated on a 5 -point scale ranging from $0=$ only internal to $4=$ only external sources).

\section{Awareness factors}

For detailed information on the questions, see Table 2.

Awareness knowledge was measured by asking if subjects had ever heard of the C-MIS before reading the introduction to the questionnaire explaining the C-MIS (answering categories "yes"/ "no").

Personal relevance was measured on a 3-item scale. Items were: "It is important that cardiac patients receive assistance with smoking cessation", "It is important that cardiology wards assist patients with smoking cessation" and "It is important that cardiology wards have protocols on how to assist patients with smoking cessation". A 5point Likert answering scale, ranging from $-2=$ totally disagree to $2=$ totally agree was 
used for each item. For the "personal relevance" subscale, the three item scores were averaged (Cronbach's $\mathrm{a}=0.95$ ).

Risk perception was assessed by asking for the perceived risks of smoking in terms of worsening cardiac disease (rated on a 10-point answering scale ranging from $1=$ no risk at all to $10=$ very strong risk) and by asking for the effect of smoking cessation on the risk of having another heart attack (rated on a 5-point scale ranging from $-2=$ risk increases $>20 \%$ to $+2=$ risk decreases $>20 \%$ ).

\section{Motivational factors}

For detailed information on the questions, see Table 3.

Attitudes about using the C-MIS were assessed by 20 questions. There were 10 questions on three of the main characteristics of innovations influencing their adoption, as defined by Rogers, namely its perceived relative advantage, compatibility, and complexity (Rogers, 1995). These questions were derived from the validated Rogers' Adoption Questionnaire developed by Steckler et al (Steckler et al., 1992), which was also used in comparable studies (Bolman et al., 2002; Mesters \& Meertens, 1999). The questions were supplemented with 10 questions on perceived health benefits and outcome expectations (Bandura, 1986) of using the C-MIS protocol, derived from an earlier study by Bolman and colleagues (Bandura, 1986; Bolman et al., 2002). All attitude questions were to be rated on a 5-point Likert answering scale, ranging from strongly disagree $(-2)$ to strongly agree $(+2)$. A factor analysis with oblimin rotation on the attitude variables revealed two attitude factors: advantages (10 items; Cronbach's $\mathrm{a}=0.92$ ) and disadvantages (10 items; Cronbach's $\mathrm{a}=0.88$ ) of using the C-MIS protocol. Scale scores were calculated by averaging the scores of the items loading high on the factor.

Social influence was assessed by using three constructs, namely support, norms, and modeling. Perceived support and norms were assessed for six different subgroups (nurses, cardiologists, patients, hospital board, other wards in the hospital, and other wards outside the hospital). For each subgroup, support was measured by the question "Do you perceive support or discouragement from ... to work with the C-MIS" (rated on a 5 -point scale ranging from $-2=$ much discouragement to $+2=$ much support).

Subsequently, two scales for support were developed; one scale was calculated as the average of the scores of items relating to support in the proximal social environment (nurses, cardiologists, patients (Cronbach's $a=0.62)$ ) and one by averaging the scores of items relating to support in the distal social environment (hospital board, other wards within and outside the hospital (Cronbach's $a=0.83)$ ). For all subgroups, norms were measured by means of the question "Do you perceive that ... think your ward should support patients with the C-MIS." (rated on a 5-point scale ranging from -2, absolutely 
not, to +2 , absolutely). For norms, a proximal (Cronbach's $a=0.82$ ) and a distal (Cronbach's $a=0.88$ ) "norm scale" were calculated by averaging the group scores. Modeling was assessed by asking for the perceived number of other Dutch cardiology wards that work with the C-MIS (rated on a 5 -point scale ranging from $0=$ none to $4=$ all).

Self-efficacy expectations were measured by asking for three aspects of introducing the C-MIS at the ward, if the ward was able to manage them: getting all nurses on the ward to work according to a protocol, getting all nurses on the ward trained in using the C-MIS, and having the nurses on the ward support all smoking cardiac inpatients with smoking cessation (rated on a 5-point scale ranging from $-2=$ no, definitely not to $+2=$ yes, definitely). The self-efficacy scale was calculated by averaging the scores for the various aspects of introducing the C-MIS at the ward (Cronbach's standardized $a=0.70)$.

\subsubsection{Analyses}

Wards were classified into two groups: adopters and non-adopters. First, t-tests or chisquare tests were conducted to test differences between adopters and non-adopters, with respect to all factors in our research model (see Figure 1). Chi-square tests were used for dichotomous variables (gender, being a smoker, type of hospital, patient- or task-oriented). Second, Spearman correlations were computed between the significant determinants of adoption and the variable of adoption itself. Third, to identify the main predictors of adoption (see Figure 1), three logistic regression analyses were performed, using backward likelihood procedures $\left(p_{\text {in }}=0.05 p_{\text {out }}=0.10\right)$. The dependent variable in the regression model was adoption. In the first analysis, the predisposing factors were analyzed to find out which predisposing variables were the main predictors of adoption. In the second analysis, awareness variables were added to the predisposing variables. In the third regression analysis, attitude, social influence and self-efficacy concepts were added to the previous model, to identify which variables were the main predictors of adoption. Because of the large number of variables relative to the number of respondents, we were not able to include all variables in the logistic regression analysis, so our analysis included only variables with $p<0.20$ in the t-test or chi-square test (Tabachnick \& Fidell, 2001). Assumptions for regression analysis and a check on multicollinearity between the independent variables were found to be satisfactory. Differences were considered significant when $p<0.05$. All analyses were performed using SPSS version 12.0.1. 


\subsection{Results}

\subsubsection{Respondent characteristics}

Of the 121 ward heads who received a questionnaire, 77 (64\%) responded. One questionnaire was excluded from further analysis because $90 \%$ of the data was missing, reducing the total sample to 76 respondents. The sample consisted of $37 \%$ men $(n=28)$ and $63 \%$ women $(n=48)$. The mean age was $38(S D=9)$. The ward heads had been working on the wards for an average of 7 years $(S D=6)$ and had been working in their current profession (mostly as a nurse) for an average of 15 years $(S D=9)$. A remarkable finding was that only $13 \%$ of the ward heads $(n=10)$ who returned the questionnaire were smokers. The C-MIS had been adopted by $63 \%$ of the wards.

Table 1. Means of and significant differences in predisposing factors for adopters and non-adopters ${ }^{1}$

\begin{tabular}{|c|c|c|c|c|}
\hline Variable (scale) & $\begin{array}{l}\text { Overall } \\
(N=76)\end{array}$ & $\begin{array}{l}\text { Adopters } \\
(n=48)\end{array}$ & $\begin{array}{l}\text { Non-adopters } \\
(n=28)\end{array}$ & $\underset{\$}{p-v a l u e ~}$ \\
\hline \multicolumn{5}{|l|}{ Environmental characteristics } \\
\hline Innovativeness of hospital board $(-2 /+2)$ & 0.79 & 0.77 & 0.82 & \\
\hline University hospital (\% yes) & 7 & 4 & 11 & @ \\
\hline \multicolumn{5}{|l|}{ Organizational characteristics } \\
\hline Sufficient employees $(0 / 4)$ & 2.55 & 2.58 & 2.50 & \\
\hline Sufficient financial resources $(0 / 4)$ & 2.22 & 2.29 & 2.11 & \\
\hline Number of nurses & $29(\mathrm{SD}=13)$ & $31(\mathrm{SD}=12)$ & $24(S D=15)$ & $<0.05$ \\
\hline Number of beds & $29(\mathrm{SD}=11)$ & $31(\mathrm{SD}=11)$ & $27(\mathrm{SD}=12)$ & \\
\hline Innovativeness of "ward" team $(-2 /+2)$ & 0.63 & 0.71 & 0.50 & \\
\hline Patient-oriented nursing system (\% yes) & 79 & 81 & 75 & $*$ \\
\hline \multicolumn{5}{|l|}{ Characteristics of decision-maker } \\
\hline Gender (\% female) & 65 & 63 & 68 & $*$ \\
\hline Age & $38(\mathrm{SD}=9)$ & $38(\mathrm{SD}=9)$ & $37(S D=8)$ & \\
\hline Smoker & 13 & 8 & 21 & @ \\
\hline Years of working in current profession & $15(S D=9)$ & $16(\mathrm{SD}=9)$ & $15(\mathrm{SD}=10)$ & \\
\hline Years of working on current ward & $7(\mathrm{SD}=6)$ & $7(S D=6)$ & $7(S D=6)$ & \\
\hline Innovativeness of ward head $(-2 /+2)$ & 1.20 & 1.21 & 1.18 & \\
\hline \multicolumn{5}{|l|}{ Decision-making styles $(0 / 4)$} \\
\hline Centralization & 1.47 & 1.50 & 1.43 & \\
\hline Formalization & 2.26 & 2.08 & 2.57 & $<0.05$ \\
\hline Amount of information used & 3.04 & 3.13 & 2.89 & \\
\hline $\begin{array}{l}\text { Proportion of external information sources vs. } \\
\text { internal information sources }\end{array}$ & 1.86 & 1.85 & 1.86 & \\
\hline
\end{tabular}

${ }^{1}$ The value in the table is the mean value unless indicated otherwise $\$ p$-values for differences between means were tested with t-test unless indicated otherwise, and are only presented if significant $*$ Differences between the groups were tested with chi-square tests @ Fisher's exact tests instead of Chi-square tests were used, because of the small number of cases in one group (e.g. the small number of ward heads who smoked $(n=10)$ and the small number of university hospitals $(n=5)$ ) 


\subsubsection{Differences between adopters and non-adopters}

\section{Predisposing factors}

Table 1 shows that adopters $(n=48)$ had a significantly lower level of formalization than non-adopters $(n=28)$, and that significantly more nurses were working on the adopting wards. Adopters and non-adopters did not differ significantly on any of the other predisposing factors.

\section{Awareness factors}

Table 2 shows that $98 \%$ of the adopters had heard of the C-MIS before they received the questionnaire. One ward head decided to adopt the C-MIS based only on the introduction to the questionnaire. Significantly fewer non-adopters had heard of the C-MIS before they received the questionnaire. There were no significant differences in risk perception and personal relevance.

Table 2. Means of and significant differences in awareness, for adopters and nonadopters $^{1}$

\begin{tabular}{|c|c|c|c|c|}
\hline Variable (scale) & $\begin{array}{l}\text { Overall } \\
(\mathrm{N}=76)\end{array}$ & $\begin{array}{l}\text { Adopters } \\
(n=48)\end{array}$ & $\begin{array}{l}\text { Non- } \\
\text { adopters } \\
(\mathrm{n}=28)\end{array}$ & $\underset{\$}{p-v a l u e ~}$ \\
\hline \multicolumn{5}{|l|}{ Knowledge } \\
\hline Ever heard of C-MIS before (\% yes) & 84 & 98 & 61 & $<0.001^{*}$ \\
\hline \multicolumn{5}{|l|}{ Risk perception } \\
\hline Estimated risk of smoking for worsening cardiac disease $(1 / 10)$ & 9.36 & 9.33 & 9.39 & \\
\hline $\begin{array}{l}\text { Estimated relative risk of smoking compared to other risk factors } \\
\text { for worsening cardiac disease }\end{array}$ & 1.15 & 1.15 & 1.16 & \\
\hline Personal relevance (agreement $-2 /+2$ ) & 1.38 & 1.50 & 1.18 & \\
\hline Supporting cardiac patients to quit smoking is important. & 1.41 & 1.50 & 1.25 & \\
\hline $\begin{array}{l}\text { Supporting patients to quit smoking by cardiology wards is } \\
\text { important. }\end{array}$ & 1.34 & 1.48 & 1.11 & \\
\hline $\begin{array}{l}\text { Availability of a protocol on stop-smoking support to } \\
\text { patients at cardiology wards is important. }\end{array}$ & 1.38 & 1.52 & 1.14 & \\
\hline
\end{tabular}

\footnotetext{
${ }^{1}$ The value in the table is the mean value unless indicated otherwise $\$ p$-values for differences between means were tested with t-test unless indicated otherwise * Differences between the groups were tested with chisquare tests
}

\section{Motivational factors}

In general, both adopters and non-adopters had a positive attitude towards the C-MIS. Both groups perceived more advantages than disadvantages of using the C-MIS. Table 3 shows that adopters were significantly more convinced of this than non-adopters. Adopters were significantly more positive about almost all attitudinal aspects (feasibility, simplicity, relative advantage, and positive outcomes of the protocol), and more convinced of the absence of disadvantages. 
Table 3. Means of and significant differences in motivational factors, for adopters and non-adopters

\begin{tabular}{|c|c|c|c|c|}
\hline Variable (scale) & $\begin{array}{l}\text { Overall } \\
(\mathrm{N}=76)\end{array}$ & $\begin{array}{l}\text { Adopters } \\
(n=48)\end{array}$ & $\begin{array}{c}\text { Non- } \\
\text { adopters } \\
(\mathrm{n}=28)\end{array}$ & $\begin{array}{c}\mathrm{p}- \\
\text { value }\end{array}$ \\
\hline \multicolumn{5}{|l|}{ Attitude items (agreement -2/+2) } \\
\hline Advantages & 1.16 & 1.36 & 0.91 & $<0.001$ \\
\hline \multicolumn{5}{|l|}{ The C-MIS protocol ... } \\
\hline ...improves the overall quality of our smoking cessation education & 1.36 & 1.63 & 0.89 & $<0.01$ \\
\hline $\begin{array}{l}\text {...is more effective in motivating patients to quit smoking than our } \\
\text { current or former practice }\end{array}$ & 1.14 & 1.35 & 0.89 & \\
\hline ...makes it easier for the nurses to help patients quit smoking & 1.30 & 1.58 & 0.82 & $<0.01$ \\
\hline ...improves the preventive task of our ward & 1.55 & 1.67 & 1.36 & $<0.05$ \\
\hline ...is a useful tool to help patients think about the pros of quitting & 1.04 & 1.23 & 0.71 & $<0.05$ \\
\hline ...is a useful tool to motivate patients to stop smoking & 1.20 & 1.44 & 0.79 & $<0.01$ \\
\hline ...is effective in reducing smoking by patients & 0.84 & 1.04 & 0.50 & $<0.05$ \\
\hline Using the C-MIS protocol has more advantages than disadvantages & 1.03 & 1.35 & 0.46 & $<0.001$ \\
\hline Using the C-MIS increases the no. of patients who quit successfully & 0.91 & 1.13 & 0.54 & $<0.05$ \\
\hline By using the C-MIS protocol, nurses do what they are supposed to & 1.25 & 1.48 & 0.86 & $<0.01$ \\
\hline Disadvantages & -0.71 & -1.00 & -0.39 & $<0.001$ \\
\hline The C-MIS protocol is hard to use & -0.96 & -1.31 & -0.36 & $<0.001$ \\
\hline The C-MIS protocol is hard to understand & -1.26 & -1.48 & -0.89 & $<0.01$ \\
\hline The C-MIS protocol is not feasible in daily routine & -0.80 & -1.10 & -0.29 & $<0.01$ \\
\hline The C-MIS protocol is inflexible and hard to adapt to daily routine & -0.67 & -0.90 & -0.29 & $<0.01$ \\
\hline If we use the C-MIS we use a method which is not flexible enough & -0.59 & -0.88 & -0.11 & $<0.01$ \\
\hline Using the C-MIS protocol puts too much of a burden on the staff & -0.32 & -0.46 & -0.07 & \\
\hline The C-MIS protocol limits the nurses' freedom & -0.88 & -1.19 & -0.36 & $<0.01$ \\
\hline Working with the C-MIS protocol takes too much time & -0.30 & -0.52 & 0.07 & $<0.05$ \\
\hline When using the C-MIS protocol we bother our patients & -0.67 & -0.94 & -0.21 & $<0.01$ \\
\hline Working with the C-MIS protocol costs too much money & -0.66 & -1.06 & 0.04 & $<0.001$ \\
\hline \multicolumn{5}{|l|}{ Social influence items $(-2 /+2)$} \\
\hline Perceived support in proximal social environment & 0.70 & 0.82 & 0.49 & $<0.01$ \\
\hline Nurses & 0.67 & 0.83 & 0.39 & $<0.05$ \\
\hline Cardiologists & 0.70 & 0.79 & 0.54 & \\
\hline Patients & 0.72 & 0.83 & 0.54 & \\
\hline Perceived support in distal social environment & 0.36 & 0.28 & 0.51 & $<0.05$ \\
\hline Hospital board & 0.43 & 0.38 & 0.54 & \\
\hline Other wards inside hospital & 0.41 & 0.29 & 0.61 & $<0.05$ \\
\hline Other wards outside hospital & 0.25 & 0.17 & 0.39 & $<0.05$ \\
\hline Perceived norm in proximal social environment & 0.95 & 1.23 & 0.48 & $<0.001$ \\
\hline Nurses & 1.07 & 1.38 & 0.54 & $<0.001$ \\
\hline Cardiologists & 1.04 & 1.33 & 0.54 & $<0.001$ \\
\hline Patients & 0.75 & 0.98 & 0.36 & $<0.01$ \\
\hline Perceived norm in distal social environment & 0.50 & 0.58 & 0.36 & \\
\hline Hospital board & 0.61 & 0.73 & 0.39 & \\
\hline Other wards inside hospital & 0.43 & 0.50 & 0.54 & \\
\hline Other wards outside hospital & 0.45 & 0.50 & 0.36 & \\
\hline \multicolumn{5}{|l|}{ Modeling } \\
\hline Perceived cessation assistance given by other cardiology wards $(0 / 4)$ & 1.30 & 1.44 & 1.07 & $<0.01$ \\
\hline Self-efficacy $(-2 /+2)$ & 0.79 & 0.92 & 0.55 & $<0.05$ \\
\hline Ward will succeed in having all nurses work according to a protocol & 0.96 & 1.00 & 0.89 & \\
\hline Ward will succeed in having all nurses trained to use the C-MIS & 0.97 & 1.15 & 0.68 & $<0.05$ \\
\hline $\begin{array}{l}\text { Ward will succeed in having all smoking patients supported } \\
\text { according to the C-MIS }\end{array}$ & 0.42 & 0.63 & 0.07 & $<0.05$ \\
\hline
\end{tabular}

$\$ p$-values for differences between means tested with t-test 
With respect to social influences, Table 3 reveals that both adopters and nonadopters perceived support from their social environment when introducing the C-MIS. However, adopters perceived significantly more support than non-adopters from the nurses on their ward. In contrast, non-adopters perceived more support from other wards than adopters. Adopters perceived significantly stronger positive norms among nurses, cardiologists, and patients towards providing the C-MIS than non-adopters. With regard to modeling, adopters expected significantly more other cardiology wards to be working with the C-MIS than non-adopters did.

Table 3 also shows that self-efficacy among adopters was significantly higher than among non-adopters; they were more convinced that they were able to have all nurses trained in using the C-MIS protocol and to have all smoking patients supported according to the C-MIS.

\subsubsection{Correlations}

Table 4 presents the Spearman correlation coefficients for the relations between the potential determinants of adopting the C-MIS, and between those determinants and adoption itself.

Table 4. Spearman correlations of determinants of adoption $(N=76)$

\begin{tabular}{|c|c|c|c|c|c|c|c|c|c|c|c|c|c|c|c|}
\hline & 1 & 2 & 3 & 4 & 5 & 6 & 7 & 8 & 9 & 10 & 11 & 12 & 13 & 14 & 15 \\
\hline $\begin{array}{l}1 \text { Social norm } \\
\text { proximal }\end{array}$ & 1 & & & & & & & & & & & & & & \\
\hline $\begin{array}{l}2 \text { Social norm } \\
\text { distal }\end{array}$ & $.58^{\mathrm{b}}$ & 1 & & & & & & & & & & & & & \\
\hline $\begin{array}{l}3 \text { Social support } \\
\text { distal }\end{array}$ & -.03 & $.44^{\mathrm{b}}$ & 1 & & & & & & & & & & & & \\
\hline $\begin{array}{l}4 \text { Social support } \\
\text { proximal }\end{array}$ & $.48^{\mathrm{b}}$ & $.30^{\mathrm{b}}$ & $.28^{\mathrm{a}}$ & 1 & & & & & & & & & & & \\
\hline 5 Self-efficacy & .22 & .14 & -.02 & $.40^{\mathrm{b}}$ & 1 & & & & & & & & & & \\
\hline 6 Advantages & $.58^{\mathrm{b}}$ & $.32^{\mathrm{b}}$ & .07 & $.35^{\mathrm{b}}$ & $.39^{b}$ & 1 & & & & & & & & & \\
\hline 7 Disadvantages & $-.51^{\mathrm{b}}$ & -.21 & .03 & $-.31^{\mathrm{b}}$ & $-.45^{\mathrm{b}}$ & $-.66^{\mathrm{b}}$ & 1 & & & & & & & & \\
\hline 8 Modeling & .14 & .01 & -.13 & .06 & $.37^{b}$ & $.25^{\mathrm{a}}$ & $-.24^{\mathrm{a}}$ & 1 & & & & & & & \\
\hline 9 Personal norm & $.34^{\mathrm{b}}$ & .22 & .02 & .16 & $.26^{\mathrm{a}}$ & $.50^{\mathrm{b}}$ & $-.44^{\mathrm{b}}$ & .03 & 1 & & & & & & \\
\hline $\begin{array}{l}10 \text { Number of } \\
\text { nurses }\end{array}$ & .14 & .03 & -.13 & .02 & -.08 & -.02 & -.12 & .02 & .21 & 1 & & & & & \\
\hline $\begin{array}{l}11 \text { Number of } \\
\text { beds }\end{array}$ & .02 & .01 & -.01 & .14 & .02 & -.11 & .02 & .10 & .15 & $.59^{\mathrm{b}}$ & 1 & & & & \\
\hline 12 Smoker & .04 & .20 & .12 & .04 & .16 & -.09 & .08 & -.14 & -.09 & -.16 & -.15 & 1 & & & \\
\hline 13 Formalization & -.20 & -.07 & .06 & $-.25^{\mathrm{a}}$ & -.20 & -.14 & $.23^{\mathrm{a}}$ & .00 & -.06 & -.04 & .01 & .00 & 1 & & \\
\hline $\begin{array}{l}14 \text { Amount of } \\
\text { information used }\end{array}$ & .17 & .16 & .07 & .23 & .08 & .08 & -.03 & .12 & .02 & .12 & .10 & -.03 & -.05 & 1 & \\
\hline $\begin{array}{l}15 \text { Heard of } \\
\text { C-MIS before }\end{array}$ & $.34^{\mathrm{b}}$ & .19 & -.21 & -.03 & .14 & $.26^{\mathrm{a}}$ & -.21 & .20 & .14 & $.27^{\mathrm{a}}$ & .03 & .06 & -.08 & -.03 & 1 \\
\hline 16 Adopters & $.49^{\mathrm{b}}$ & .17 & $-.25^{\mathrm{a}}$ & $.28^{\mathrm{a}}$ & $.34^{\mathrm{b}}$ & $.39^{\mathrm{b}}$ & $-.48^{\mathrm{b}}$ & $.29^{\mathrm{a}}$ & $.28^{\mathrm{a}}$ & $.32^{\mathrm{b}}$ & .21 & -.19 & $-.29^{a}$ & .18 & $.49^{\mathrm{b}}$ \\
\hline
\end{tabular}

Adoption correlated most strongly with having heard of the C-MIS before receiving the questionnaire and with the norm in the proximal social environment, followed closely by the negative correlation with disadvantages. As regards predisposing factors, adoption 
was significantly related to the number of nurses and the level of formalization. Perceived norm in the proximal social environment showed a high positive intercorrelation $(>0.5)$ with the social norm in the distal social environment and with advantage, and showed a high negative intercorrelation with disadvantage. In addition, advantage showed a high positive intercorrelation with personal relevance and a negative intercorrelation with disadvantages. The number of beds showed a high intercorrelation with the number of nurses.

\subsubsection{Regression analyses}

Results of the regression analyses are presented in Table 5. The first logistic regression analysis (entering predisposing factors) showed that the main predisposing factors that were related to adoption were the level of formalization and the number of nurses working on the ward. These factors predicted $19 \%$ of the adoption of the C-MIS. A lower level of formalization and more nurses working on the ward increased the likelihood of adoption of the C-MIS. When awareness was added to the model, the same factors were still significant, and none of the awareness factors turned out to be significant predictors. The third logistic regression analysis added the motivation factors to the predisposing and awareness factors. The motivation factors that were significantly predictive of adoption were positive perceived norms in the proximal social environment, less perceived social support from the distal social environment, and high self-efficacy. In addition to these ASE factors, the number of nurses on the ward and being a smoker were significant predictors. These factors explained 55\% of the adoption of the C-MIS. More perceived positive norms from the proximal social environment, less perceived support from the distal social environment, high level of self-efficacy, having more nurses working on the ward, and not being a smoker increased the likelihood of adoption.

Table 5. Summary of the logistic regression analysis for variables predicting the adoption of the C-MIS

\begin{tabular}{lllll}
\hline & Beta & SE B & OR (95\% CI) & p-value \\
\hline $\begin{array}{l}\text { Step 1 } \\
\quad \text { Number of nurses on ward }\end{array}$ & 0.045 & 0.021 & $1.05(1.00-1.09)$ & 0.03 \\
$\quad \begin{array}{l}\text { Level of formalization } \\
\text { Step 2 }\end{array}$ & -.850 & 0.345 & $0.43(0.21-0.86)$ & 0.02 \\
$\quad$ Number of nurses on ward & 0.045 & 0.021 & $1.05(1.00-1.09)$ & 0.03 \\
$\quad \begin{array}{l}\text { Level of formalization } \\
\text { Step 3 }\end{array}$ & -.850 & 0.345 & $0.43(0.21-0.86)$ & 0.02 \\
$\quad$ Level of formalization & 0.050 & 0.025 & $1.05(1.00-1.10)$ & 0.05 \\
$\quad$ Being a smoker & -1.897 & 0.947 & $0.15(0.02-0.96)$ & 0.05 \\
$\quad \begin{array}{l}\text { Perceived proximal social } \\
\text { norm }\end{array}$ & 1.966 & 0.535 & $7.14(2.50-20.40)$ & 0.00 \\
$\quad \begin{array}{l}\text { Perceived distal social support } \\
\text { Perceived self-efficacy }\end{array}$ & -1.691 & 0.735 & $0.18(0.04-0.78)$ & 0.02 \\
\hline
\end{tabular}

$\mathrm{R}^{2}=0.19$ for steps $1 \& 2$ and $\mathrm{R}^{2}=0.55$ for step 3 


\subsection{Discussion and Conclusion}

\subsubsection{Discussion}

The present study aimed to identify psychological and organizational determinants of the adoption of an intervention for smoking cessation guidance on cardiology wards. The study found that psychological factors are important determinants of adoption. They were found to explain the adoption of the C-MIS to a larger extent than organizational factors.

Very few organizational factors were found to be related to adoption. A low level of formalization was found to increase the chance of adoption significantly; this finding is in line with Rogers' theory, which states that formalization inhibits adoption (Rogers, 1995). A larger number of nurses working on the ward was another important determinant of adoption. This relation was not due to a larger number of nurses available per bed. The positive relation between ward size and adoption has also been found in other studies (Castle, 2001; Moch \& Morse, 1977), but those studies measured ward size by the number of beds only. No other organizational variables were significantly related to adoption. This finding may have been partly caused by the fact that our statistical tests did not have enough power, due to our relatively small sample size. It might also be explained by the fact that differences between the "organizations" were limited, because we studied a rather specific setting. A third reason might be that we did not measure all important organizational characteristics in our study.

As regards the personal background characteristics of the ward heads, only their smoking behavior was found to impede adoption. Not surprisingly, knowing that the innovation exists turned out to be a conditional term for adoption. Even after four years of dissemination, there are still Dutch cardiology wards that are unaware of the C-MIS.

In general, both adopters and non-adopters had a positive attitude towards using the C-MIS. No discouragement was perceived from the social environment when introducing the C-MIS, and self-efficacy was at least moderate. However, many significant differences were found between adopters and non-adopters. In line with behavioral psychological models like the I-Change Model (De Vries et al., 2005, 2006; De Vries. Mudde, Leijs et al., 2003), adopters were more convinced about the advantages of using the C-MIS and about the absence of disadvantages. They perceived more positive influence from their social environment towards working with the C-MIS and they had a higher level of self-efficacy. With regard to the motivational factors, the only finding that was not in line with our expectations based on the I-Change Model was the perceived social support from the distal social environment, which was less for the adopters than for the non-adopters. The social support that someone who is not ready to adopt expects from the distal environment seems to be greater than the support they actually experience when intending to engage in or actually engaging in the behavior. 
The distal social environment probably turns out to be relatively uninterested in the behavior at the ward. It might as well be that a structural lack of social support from the distal environment points to a positive self-supporting attitude, that provides the ward with sufficient perseverance to adopt new programs.

Our study was subject to certain limitations. Because of its cross-sectional design, differences between adopters and non-adopters in terms of motivation factors might be due to the experience that adopters already have with the C-MIS. In our case, a longitudinal design was impossible, because a large number of wards had already adopted the C-MIS at the time of the study. Such a longitudinal study would be necessary to confirm the predictive value of the variables identified in our study. Another limitation of the study was the $64 \%$ response rate. The proportion of smokers among ward heads found in our study $(13.3 \%)$ was much smaller than the proportion of smokers among all employees in health care (30\%) (Segaar \& Willemsen, 2003). This might indicate selection bias, meaning that non-smoking ward heads were more likely to respond than smoking ward heads. Since non-smoking ward heads seem to be more likely to adopt the C-MIS, the effect of the ward head being a smoker on adoption of the C-MIS may have been underestimated in our study. Information from the electronic implementation registration system, a system of the Dutch expert centre on tobacco control which monitors the use of the C-MIS by cardiology wards, shows that the nonresponse was about as high among wards that are using the C-MIS as among wards that are not using the protocol. This provides some justification for generalizing the situation at the responding wards to the total population of wards.

\subsubsection{Conclusion}

Despite its limitations, the present study has provided important insights into the factors that play a role in the adoption of a smoking cessation protocol in cardiology wards. Provision of basic information about an innovation remains important even after years of dissemination. Furthermore, it is very important to take psychological factors into account when studying the adoption of preventive innovations and when developing diffusion strategies. The results can help to develop research-based implementation strategies to further increase and facilitate the adoption of preventive interventions in hospital settings.

\subsubsection{Practice implications}

It is necessary to ensure that the primary condition for adoption is met, namely that the target group knows of the existence of the innovation. If it is possible to identify those who are unaware, a personal approach can be used to acquaint them with the innovation. Since higher levels of formalization inhibit adoption at hospital wards, 
implementation strategies have to be developed that fit the situation in highly formalized organizations better than current strategies do. The more specific the study setting, the less variance in organizational characteristics, and the more important it is to take psychological determinants into account. To increase the levels of adoption of preventive innovations in hospital practice, implementation programs in hospital ward settings should focus primarily on psychological characteristics. Implementation programs should aim for high self-efficacy and high perceived social norms and support for the nurses on the wards, especially in the proximal social environment. Moderate self-efficacy and moderate positive social influence are not enough. 



\section{CHAPTER 3}

Characteristics of Adherence to a MinimalContact Smoking Cessation Intervention on Cardiac Wards 


\begin{abstract}
Health promotion interventions are often underused by care practitioners and therefore often lack the intended health impact. The present study assessed the use of a smoking cessation intervention on Dutch cardiac wards and factors associated with adherent use. 94 of 206 nurses did not manage to fully apply the intervention in daily practice; they did not always provide patients with self-help guides, discuss smoking cessation aids, or provide follow-up care. Factors in our comprehensive research model (the Integrated Change Model) were associated with $52 \%$ of variance in adherence. Adherence was most likely if nurses consistently used an intervention card, perceived many advantages of the intervention, had many other nurses around them who used it and had been involved in decision-making.
\end{abstract}

\title{
Submitted as:
}

Segaar, D., Willemsen, M.C., Bolman, C. \& De Vries, H. Characteristics of Adherence to a Minimal-Contact Smoking Cessation Intervention on Cardiac Wards 


\subsection{I ntroduction}

Smoking cessation is associated with a substantially reduced mortality risk in patients with coronary heart disease (Critchley \& Capewell, 2004). Hospital admission provides an excellent opportunity to help patients quit smoking (Emmons \& Goldstein, 1992; France, Glasgow, \& Marcus, 2001; Rigotti, Munafo, Murphy, \& Stead, 2003). The Smoking Cessation Guidelines for Health Professionals (Fiore et al., 2000; Raw, McNeill, \& West, 1999) recommend that primary care teams use the Ask, Advice, Assess, Assist, and Arrange (5 A's) model to guide patients in smoking cessation. Nurse-based interventions for cardiac patients that are structured according to the $5 \mathrm{~A}$ 's model effectively increase the number of successful quit attempts (Bolman, De Vries, \& Van Breukelen, 2002a, 2002b; Narsavage \& Idemoto, 2003; Quist-Paulsen \& Gallefoss, 2003; Taylor, Houston-Miller, Killen, \& DeBusk, 1990). Yet, health promotion interventions are generally underused by health care practitioners (Bostrom \& Wise, 1994; Cranney, Warren, Barton, Gardner, \& Walley, 2001; Glasgow, Lichtenstein, \& Marcus, 2003) and, therefore, often do not have the intended health impact.

Underuse is the result of failures in the process of diffusion of innovations, which can be divided into three main stages: adoption, implementation, and maintenance (Rogers, 1995). Adoption is the decision to make full use of an innovation as the best course of action. Implementation occurs when an innovation is put into use. Maintenance is the phase in which continued use of the innovation is ensured. Increasing the complete and correct use of interventions requires knowledge of the determinants of successfully passing through the three diffusion phases. Previous studies, which predominantly focused on adoption rather than on implementation or maintenance, have identified a variety of factors that may influence the diffusion of innovations, including by the target group perceived innovation characteristics, such as relative advantage (Rogers, 1995), organizational characteristics, such as the size of the organization (Castle, 2001; Moch \& Morse, 1977), and adopter's characteristics, such as attitude (Bolman, De Vries, \& Mesters, 2002; Mesters \& Meertens, 1999). Yet, most of these studies were performed outside the field of public health. Thus, information on generalizability to other settings, such as the health care setting, is often lacking (Foy, Eccles, \& Grimshaw, 2001; Glasgow et al., 2003). Furthermore, the relative weights of these factors in comparison with other factors in explaining adherence remain unclear.

The purpose of the study reported here was to examine the diffusion of innovations among health care practitioners, specifically, the implementation by cardiac nurses in the Netherlands of the Minimal Intervention Strategy for smoking cessation for cardiac inpatients (C-MIS). A study of the factors that facilitate and impede the adoption of the C-MIS on cardiac wards has been described elsewhere (Segaar, Bolman, Willemsen, \& De Vries, 2006). 
The C-MIS is a stepwise strategy, comparable to the 5 A's model, which was developed in the Netherlands for nurses to assist their cardiac patients to quit smoking. The protocol consists of stop-smoking advice by the cardiologist, followed by a stepwise process of counseling by the nurse that is adapted to the smoker's particular stage of behavior change (Prochaska \& Velicer, 1997). The counseling activities during hospitalization consist of assessing the smoker's profile, enhancing or reinforcing the motivation to quit, addressing barriers and high-risk situations, and setting a date to quit. After discharge, follow-up support is provided in the form of a telephone call by a nurse and/or of advice during an outpatient check-up by the cardiologist. Nurses record the use of C-MIS on an intervention report form, which also serves as a schematic summary of the protocol. The C-MIS has been found to be more effective than usual care in achieving smoking cessation at three months' follow-up (Bolman, De Vries, Van Breukelen, 2002a) and has been disseminated to Dutch cardiology wards nationwide since 1999. At the time of our study, the C-MIS had been implemented in 39 of the 121 Dutch cardiology wards (32\%). In these wards, nurses were trained in C-MIS skills, and supplementary educational materials (e.g. manuals for nurses and information brochures for patients) were distributed. A previous Dutch pilot study among nurses on cardiac wards showed that after implementation of the C-MIS on the ward $51 \%$ of the nurses on those wards supported the majority or all of their patients with the C-MIS (Bolman, De Vries, \& Mesters, 2002). That study however, did not look at the quality of the use of the C-MIS or the actual performance of all aspects of the C-MIS, and only assessed for a small number of factors their associations with implementation.

In this study implementation was defined to be successful when the target behavior is reached. With respect to implementation of the C-MIS, this implied full use of or adherence to the C-MIS protocol. Accordingly, our study focussed on adherence with the C-MIS. The first major objective of our study was to assess adherence to the C-MIS protocol by individual nurses and to identify which steps of the protocol were more difficult to apply than others. The second major objective was to test differences between adherent and nonadherent users of the C-MIS in terms of both organizational factors, such as number of beds and level of formalization, and psychosocial factors, such as awareness, perceived advantages and self-efficacy. Secondary objectives were to assess the applicability of the model that we used to identify potential determinants of adherence (i.e. the Integrated Change Model, or I-Change Model) (De Vries, Mesters, Van de Steeg, \& Honing, 2005; De Vries, Mudde, Leijs et al., 2003; De Vries, Mesters, Van 't Riet, Willems, \& Reubsaet, 2006) and the level of association between the factors in the I-Change Model and adherence. The model is described in the next section. 
The I-Change Model

We used the I-Change Model (De Vries et al., 2005; De Vries, Mudde, Leijs et al., 2003;

De Vries, Mesters, Van 't Riet et al., 2006) to identify potential determinants of adherence to the C-MIS.

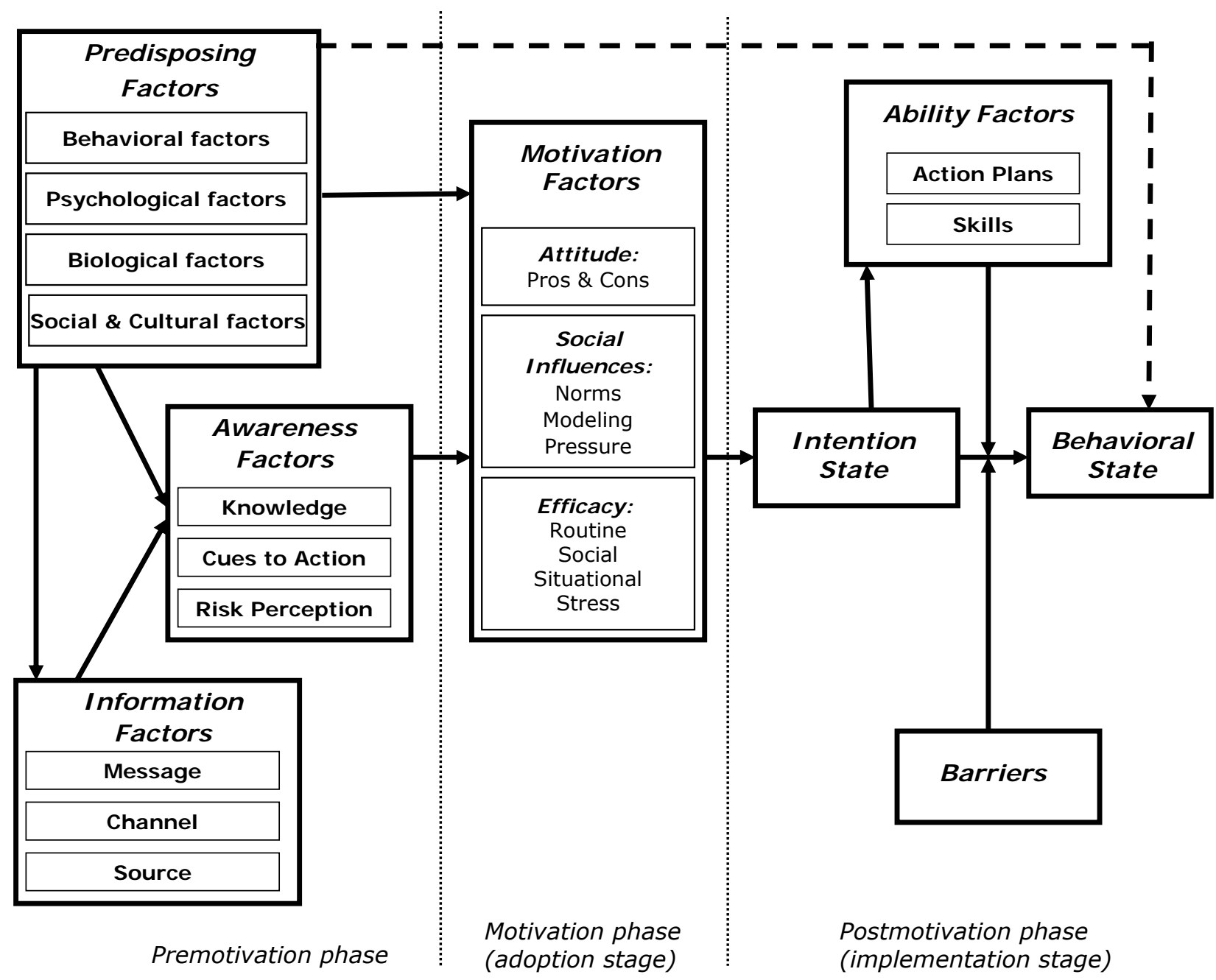

Figure 1: The I-Change Model (De Vries et al., 2005, 2006; De Vries, Mudde, Leijs et al., 2003).

This model builds on an earlier model, referred to as the Attitude - Social Influence Self Efficacy (ASE) model, and integrates concepts of various social-cognitive models, such as the Social Cognitive Theory (Bandura, 1986), the Theory of Planned Behavior (Ajzen, 1991), and the Transtheoretical Model (Prochaska \& DiClemente, 1983).

The I-Change Model shown in Figure 1 distinguishes three phases in behavioral change processes: (a) a pre-motivation phase, in which a person becomes aware of a problem and the need for new or altered behavior; (b) a motivation phase, in which the 
intention to engage in the new behavior is formed; and (c) a post-motivation phase, in which this intention is transformed into behavior.

The I-Change Model posits that behavior is the result of a person's intentions, abilities, and barriers. Intentions are, in turn, determined by attitudes, perceived social influences, and self-efficacy. Attitude is assumed to be the result of weighing the perceived advantages (pros) and disadvantages (cons) of the behavior. In diffusion studies, it includes beliefs about the innovation characteristics defined by Rogers (2002), namely, perceived relative advantage, compatibility, complexity, trialability, and visibility of results. Social influences consist of perceiving others who engage in the behavior (modeling), the perceived norms of others, and the perceived support encountered from others. Self-efficacy refers to one's perception of one's ability to engage in the behavior.

The I-Change Model assumes that these motivational factors are determined by various pre-motivational factors, namely, awareness factors (e.g. knowledge, cues to action), information factors (e.g. channel, source), and predisposing factors (e.g. behavioral factors, psychological factors). For the purpose of our study, we assessed all factors (premotivation, motivation, and postmotivation) of the model. The predisposing factors on which our study mainly focused were organizational characteristics of the hospital ward, such as organizational context and decision-making style (Koopman \& Pool, 1991, 1994). These were selected because the organization plays an important role in creating the conditions to use an intervention.

\subsection{Methods}

A cross-sectional survey was conducted among nurses of cardiac inpatients wards, 5 years after the start of the nationwide dissemination of the C-MIS. At that time 39 of the 121 cardiac wards implemented the C-MIS. On patient-oriented wards this meant that all registered nurses on the wards within the previous 5 years attended the C-MIS skills training or watched the instruction video and read the instruction manual. Nurses who started to work on the ward when the training had already been given only watched the instruction video and read the manual. In the exceptional case of task-oriented wards, a few nurses were designated to provide the C-MIS and those nurses attended the skills training and read the manual. The C-MIS is mainly performed by the nursing staff, therefore study subjects were nurses who worked in cardiology wards that had implemented the C-MIS during the nationwide dissemination campaign. For the purpose of this study, a random sample of 25 wards was drawn from all 39 Dutch cardiology wards that had implemented the C-MIS. Not all 39 wards were included due to practical and financial considerations. Ward heads of the selected wards were asked if they were willing to participate in our study. If yes, they were asked to give all registered nurses on their wards a questionnaire and ask them to complete it. Twenty heads of wards 
( $80 \%)$, with a total of 553 nurses, agreed to participate. Nurses could return the questionnaire anonymously. Respondents could win a voucher worth $€ 25$ if they returned the questionnaire. Reminders were sent to the ward heads three weeks later, accompanied by a reminder poster. Nurses who worked on a task-oriented ward were excluded from our study if they were not among the nurses that were designated to perform the C-MIS on their ward.

\subsubsection{Questionnaire}

The questionnaire was based on existing questionnaires or relevant scales, information from the literature, and earlier studies on health promotion interventions, of which several focussed on smoking cessation support by health professionals (Bolman et al., 2002; Cooke, 2000; Mesters \& Meertens, 1999; Mudde, Willemsen, Kremers, \& De Vries, 2000; Pieterse, 2001; Segaar et al., 2006; Steckler, Goodman, McLeroy, Davis, \& Koch, 1992). The questionnaire consisted of 155 forced-choice questions. Most of these were part of scales (e.g. the pros-scale consisted of 14 items). The various measures are described below and in Tables 1 to 5 . Overall scores per scale were calculated by averaging the scores of items that measured the same variable, unless indicated otherwise. If scales were calculated, we present Cronbach's a (three or more items) or the correlation coefficient $r$ (two items). Most answering scales, with respect to questions other than on predisposing and information factors, were 5-point Likert scales. If other types of scales were used this is indicated as part of the question description.

\section{Outcome Variable}

The outcome variable, adherence to the C-MIS, was assessed by nine questions about compliance with elements of the C-MIS. In a previous phase of the study, the relevant elements of the MIS had been identified by a group of five experts in the fields of health psychology, health education, and smoking. Nurses were asked to indicate how often ( 0 =never, 4 =always) they applied the nine elements of the C-MIS. A total score for adherence was calculated by adding up the nine scores. In accordance with the views of an expert panel, which concluded that striving to use a guideline all of the time might be too much to expect but most of the time should be the goal (Gross et al., 2001), a cutoff point was defined at three quarters of the maximum value of 36 . If the score was 27 or more, nurses were categorized as "adherent users"; otherwise they were classified as "nonadherent users".

\section{Premotivational Factors}

Detailed information on the questions about predisposing factors is provided in Table 1. 
The biological factors we assessed included age and sex of the nurse. Behavioral factors assessed were nurses' own smoking behavior (Mudde et al., 2000), the number of years they had been employed as a nurse, the number of years they had been employed at the current ward, the number of working hours per week, and their personal level of innovativeness. Social and cultural factors were assessed with questions related to organizational context, decision-making style, and the involvement of staff members in decision-making. Organizational context characteristics included ward size (number of cardiologists and nurses working on the ward and number of beds on the ward), availability of a smoking room on the ward for either nurses or patients, frequency with which the C-MIS was discussed in team meetings, number of smoking patients nursed per month, workload, and the nursing system (patient-oriented or task-oriented). Style of decision-making was assessed by four items (Koopman \& Pool, 1991, 1994): centralization (whether decisions were entirely made by the individual nurses or entirely centralized at ward level); formalization of decision-making (own solutions or set procedures); amount of information gathered before decisions were made and the proportion of external information sources versus internal information sources consulted. Involvement in decision-making was assessed by three questions (5-point likert scale, Cronbach's $a=0.80)$.

Detailed information on the questions about information factors is provided in Table 1. The nurses were asked if they consulted each of six sources of information (implementation training, skills training, colleagues, instructions manual, instruction video and newsletter) indicated in the questionnaire. Since the construct was designed to assess the total number of information sources together, it was regarded as an index instead of a scale. The index for information factors was calculated by counting the number of information sources used.

Detailed information on the questions about awareness is provided in Table 2. Knowledge of the C-MIS was measured by two questions $(r=0.75)$ on the level of knowledge on the C-MIS, i.e. "Do you know what the C-MIS is?" and "Are you familiar with all steps of the C-MIS?" (10-point ratio $0=$ not at all, $4=$ very well). Cues to action were measured by two subscales: personal relevance ( 6 items, Cronbach's $a=0.77$ ) and dissatisfaction ( 2 items, $r=0.62$ ) with the current method of smoking cessation guidance. Risk perception was assessed by asking two independent questions, one about the perceived risk of smoking in terms of worsening cardiac disease (5-point ordinal scale $1=$ no risk at all, $10=$ very high risk) and by asking for the effect of smoking cessation on the risk of having another heart attack ( $-2=$ decreases, $2=$ increases). 


\section{Motivational Factors}

Attitudes (Table 3 ) were assessed by 24 questions, including 12 items on beliefs about the innovation characteristics defined by Rogers, i.e. relative advantage, compatibility, complexity, trialability and observability (Rogers, 2002; Steckler et al., 1992), and 12 questions on perceived health benefits and other outcome expectations of using the $C$ MIS protocol (Bolman et al., 2002; Segaar et al., 2006). A factor analysis with oblimin rotation on the attitude items revealed two attitude factors: the pros (14 items, Cronbach's $\mathrm{a}=0.88$ ) and the cons (10 items, Cronbach's $\mathrm{a}=0.87$ ) of using the C-MIS protocol. Attitude ambivalence (Ajzen, 2001) was measured by two questions ( $r=0.86$ ) i.e., "I am not sure about my opinion regarding the C-MIS" and "I doubt whether I think that I should work with the C-MIS".

Perceived social influences (Table 4) were assessed using three constructs: social norms, social support, and modeling (De Vries, Backbier, Kok, \& Dijkstra, 1995). Perceived norms and support were assessed for four subgroups, i.e. nurses, ward heads, cardiologists and patients. Since both constructs were designed to assess the perceived opinions and behavior of several persons together, and the correlation between the items may, therefore, be low, they were regarded as indexes instead of scales, and the scores of the subgroups were summed. Social modeling was assessed by one question with respect to the expected proportion of nurses on the own ward one expects to use the C-MIS (0\%-100\%).

Self-efficacy expectations (Table 4) were measured by asking nurses whether they would succeed in providing smoking cessation guidance with the C-MIS in 12 situations occurring in daily practice. Situations were derived from previous research on the adoption of smoking cessation programs in health care (Bakker, Mullen, De Vries, \& Van Breukelen, 2003; Cooke, Mattick, \& Campbell, 2000). A 7-point Likert scale was used. Factor analysis revealed five self-efficacy factors. The first one is stress selfefficacy ( 3 items, Cronbach's a=0.70), this includes a busy ward, reorganization and working with substitutes for ill colleagues. The second one is situational self-efficacy with respect to resistant patients ( 2 items, $r=0.66$ ), this includes lack of interest and resistance of patient. The third one is situational self-efficacy with respect to patient characteristics ( 2 items, $r=0.52$ ), this includes educational level and language problems. The fourth one is routine self-efficacy ( 3 items, Cronbach's $a=0.89$ ), this includes cases of irregular and infrequent use. The last one is social self-efficacy ( 2 items, $r=0.44$ ), this includes behavior of colleagues and coordination. Overall scores per scale were calculated by averaging the scores of the items with the highest loading on the factors.

\section{Postmotivational Factors}

Detailed information on the questions on ability factors and barriers is provided in 
Table 5. First, perceived skills were assessed by asking nurses if they thought they had sufficient skills to implement seven aspects of the C-MIS (Cronbach's $a=0.90$ ).

Second, as actions plans are difficult to measure retrospectively, we measured the related concept goal actions, which can be defined as "sub-actions" that faciltate the achievement of the intended final goal (behavior). Goal actions were assessed by asking nurses how often they performed six actions that facilitated the use of the C-MIS ( $0=$ never, $4=$ very often), and by asking how frequently nurses used the C-MIS intervention card when applying the C-MIS ( $0=$ never, $4=$ always). Factor analysis with oblimin rotation on the seven goal action items revealed three factors; general goal actions ( 3 items , Cronbach's a $=0.79$ ), situation specific goal actions ( 3 items, Cronbach's $a=0.56)$ and intervention card use ( 1 item).

The only barrier we assessed was the availability of two essential types of material that were essential to carry out the C-MIS properly (yes/no). An index was calculated by adding up the two scores.

\subsubsection{Statistical Analysis}

With respect to the first main study objective, descriptive statistics were used to assess adherence to the C-MIS protocol and to assess which steps of the protocol were the most difficult ones to implement. With respect to the second main study objective, chisquare tests and t-tests were used to test differences between adherent users and nonadherent users for all factors in our research model (see Figure 1). Pearson correlations with adherence were computed for all variables.

With respect to our secondary objective, to assess the applicability of the IChange model in research on protocol use by nurses, a sequential logistic regression analysis was conducted. The dependent variable was adherence with the C-MIS (yes/no) and the independent variables were all premotivational, motivational and postmotivational factors (see Tables 1-5 and previous subsection). Scale scores were always included, with the exception of two index subscales, two sumscore subscales and a few individual items that were not compound. For the regression analysis the few missing data were replaced with the mean. The results of this analysis indicated to what extent the factors in the I-Change Model were associated with adherence to the C-MIS protocol. Because of the sequential procedure, we also assessed if motivational factors added significant explanatory value to premotivational factors and if they mediated the association between premotivational factors and behavior, as assumed in the I-Change model. Furthermore, we examined whether postmotivational factors added significant value to premotivational and motivational factors in predicting adherence. First, the premotivational factors (predisposing, information, and awareness factors), were entered into the model. Then, motivational factors were added to the model, using the 
enter procedure and, in the third block, the postmotivational factors (ability factors and barriers) were added by the enter procedure.

Because of the large number of variables relative to the number of respondents, we were unable to include all variables in the logistic regression analysis, so our analysis included only those variables with $p<0.20$ in the t-test or Chi-square test (see Tables 15; Tabachnick \& Fidell, 2001). Standardized Z-scores of the variables were used to compare the magnitude of the partial association of variables with adherence, based on their standardized beta values $(\beta)$. Nagelkerke's $R^{2}$ was used to approximate the total levels of association with adherence.

Assumptions for regression analysis were met, and a check on multicollinearity between the independent variables was satisfactory. Differences were considered significant at $p<0.05$. All analyses were performed with SPSS version 13.0.

\subsection{Results}

\subsubsection{Sample Description}

A total of 210 of the 553 nurses (38\%) returned the questionnaire. One questionnaire was excluded from further analyses since no answers had been provided on the outcome variable use of the C-MIS. Three nurses worked on a task-oriented ward where other nurses were responsible for the C-MIS, so it was not part of their job to use it. They were, therefore, excluded. Of the remaining 206 nurses, 94 (46\%) were nonadherent users of the C-MIS and 112 (54\%) were adherent users.

\subsubsection{Adherence}

About $8 \%(n=17)$ of the nurses never performed any step of the C-MIS. About $80 \%$ of the nurses assessed smoking status $(n=167)$, the number of cigarettes smoked per day $(n=162)$, and the motivation to quit $(n=160)$ of most of their patients $(>75 \%)$. About $70 \%$ of the nurses discussed reasons to quit $(n=148)$, discussed the problems that impeded quit attempts $(n=142)$, and set a date for quitting with most of their patients $(n=140)$. Fewer nurses provided most of their smoking patients with the self-help guide $(n=120)$ or discussed the use of smoking cessation aids with motivated patients $(n=122)$, but still about $60 \%$ performed these steps with most of their patients. Almost half of all nurses $(42 \%, n=86)$ never performed follow-up care and only $36 \%$ phoned most of the patients with whom they had set a quit date after discharge $(n=75)$.

\subsubsection{Differences Between Adherent and Nonadherent Users of the C-MIS Protocol.}

The differences between adherent and nonadherent users in terms of the factors in the I-Change Model are presented in Tables 1 to 5. 
Table 1. Means of and significant differences between nonadherent and adherent users, in terms of predisposing and information factors ${ }^{1}$

\begin{tabular}{|c|c|c|c|c|}
\hline Variable (scale) & $\begin{array}{l}\text { Overall } \\
(N=206)\end{array}$ & $\begin{array}{c}\text { Nonadherent } \\
\text { users } \\
(n=94)\end{array}$ & $\begin{array}{c}\text { Adherent } \\
\text { users } \\
(n=112)\end{array}$ & $\mathrm{p}$-value \\
\hline \multicolumn{5}{|l|}{ Biological factors } \\
\hline Sex: female (\% yes) & 89 & 93 & 87 & $*$ \\
\hline Age in years & 36 & 34 & 38 & $<0.01$ \\
\hline \multicolumn{5}{|l|}{ Behavioral factors } \\
\hline Smoking behavior: smoker (\% yes) & 12 & 16 & 8 & $@$ \\
\hline Years employed as a nurse & 13 & 11 & 15 & $<0.05$ \\
\hline Years employed as a nurse on current ward & 7 & 6 & 8 & \\
\hline Number of working hours per week & 29 & 29 & 28 & \\
\hline $\begin{array}{l}\text { Personal level of innovativeness (change: }-2=\text { negative },+2= \\
\text { positive) }\end{array}$ & 0.93 & 0.85 & 1.00 & \\
\hline \multicolumn{5}{|l|}{ Social and cultural factors } \\
\hline \multicolumn{5}{|l|}{ Organization (context) } \\
\hline Number of cardiologists & 6 & 6 & 6 & \\
\hline Number of nurses & 30 & 30 & 30 & \\
\hline Number of beds & 30 & 31 & 29 & \\
\hline Smoking room for patients available (\%) & 22 & 23 & 20 & $*$ \\
\hline Smoking room for nurses available (\%) & 23 & 32 & 16 & $<0.01^{*}$ \\
\hline $\begin{array}{l}\text { Frequency with which the C-MIS is discussed in team } \\
\text { meetings ( } 0=\text { never, } 3=\text { once a month) }\end{array}$ & 1.32 & 1.24 & 1.38 & \\
\hline Number of smoking patients nursed per month & 7 & 8 & 7 & \\
\hline Workload $(-3=$ too low,$+3=$ too high $)$ & 0.73 & 0.66 & 0.79 & \\
\hline Nursing system - patient oriented (\% yes) & 74 & 71 & 78 & $*$ \\
\hline Centralization level & 2.28 & 2.32 & 2.23 & \\
\hline Formalization level & 2.45 & 2.43 & 2.45 & \\
\hline Amount of information used & 3.06 & 2.84 & 3.24 & $<0.05$ \\
\hline $\begin{array}{l}\text { Number of external information sources versus internal } \\
\text { sources }\end{array}$ & 1.46 & 1.44 & 1.52 & \\
\hline I nvolvement in decision making $(-2=$ disagree,$+2=$ agree $)$ & -0.31 & -0.60 & -0.08 & $<0.01$ \\
\hline I was involved in the decision of the ward to use the C-MIS & -0.62 & -0.97 & -0.36 & $<0.01$ \\
\hline $\begin{array}{l}\text { The majority of the ward team was involved in the decision } \\
\text { to use the C-MIS }\end{array}$ & 0.01 & -0.22 & 0.22 & $<0.01$ \\
\hline $\begin{array}{l}\text { In the decision-making process about the C-MIS I was asked } \\
\text { to give my opinion }\end{array}$ & -0.33 & -0.62 & -0.10 & $<0.01$ \\
\hline Information factors (index $0-6)^{\dagger}$ & 3.39 & 2.91 & 3.79 & $<0.01$ \\
\hline Implementation training (\% yes) & 53 & 44 & 60 & $<0.05^{*}$ \\
\hline Skills training ( $\%$ yes) & 56 & 49 & 61 & $*$ \\
\hline Colleague (\% yes) & 80 & 73 & 86 & $<0.05^{*}$ \\
\hline Skills manual (\% yes) & 76 & 67 & 84 & $<0.01 *$ \\
\hline Instruction video (\% yes) & 52 & 47 & 57 & $*$ \\
\hline Newsletter about the C-MIS (\% yes) & 31 & 21 & 39 & $<0.01 *$ \\
\hline
\end{tabular}

${ }^{1}$ The value in the table is the mean value unless indicated otherwise ${ }^{\$} p$-values for differences between means were tested with t-tests unless indicated otherwise, and are only presented if significant $*$ Differences between the groups were tested with chi-square tests ${ }^{\circledR}$ Fisher's exact tests instead of Chi-square tests were used, because of the small number of cases in one group ${ }^{\dagger}$ sum of scores (yes $=1$ ) on information factors

\section{Premotivational Factors}

With respect to the predisposing factors, Table 1 shows that adherent users of the C-MIS were significantly older than nonadherent users (i.e. 4 years) and that they had been working in their profession significantly longer (4 years). Adherent users were less likely 
to work on wards where there was a designated smoking room for nurses, and they were more likely to have been involved in the decision to adopt the C-MIS at the ward. Table 1 shows that adherent users of the C-MIS had generally received significantly more information on how to use the intervention than nonadherent users; in particular, a significantly larger proportion reported to have read the manual ( $84 \%$ vs. $67 \%$ ) and the newsletter about the C-MIS (39\% vs. $21 \%$ ).

With respect to awareness factors, Table 2 shows that adherent users had significantly more knowledge about the C-MIS (about 1.2 times higher) and that they were more convinced of the importance of smoking cessation support by nurses (personal relevance) than nonadherent users.

Table 2. Means of and significant differences in awareness factors, for adherent and nonadherent users

\begin{tabular}{|c|c|c|c|c|}
\hline Variable (scale) & $\begin{array}{c}\text { Overall } \\
(N=206)\end{array}$ & $\begin{array}{c}\text { Nonadherent } \\
\text { users } \\
(n=94)\end{array}$ & $\begin{array}{c}\text { Adherent } \\
\text { users } \\
(n=112)\end{array}$ & $\begin{array}{c}\mathrm{p}- \\
\text { value }\end{array}$ \\
\hline Knowledge level ( $0=$ not at all, $4=$ very well) & 3.21 & 2.93 & 3.46 & $<0.01$ \\
\hline Do you know what the C-MIS is? & 3.36 & 3.18 & 3.53 & $<0.01$ \\
\hline Are you familiar with all steps of the C-MIS? & 3.06 & 2.68 & 3.39 & $<0.01$ \\
\hline Cues to action: Personal relevance $(-2=$ disagree,$+2=$ agree $)$ & 1.21 & 1.08 & 1.31 & $<0.01$ \\
\hline $\begin{array}{l}\text { Giving up smoking is the most important way to limit the risk } \\
\text { of worsening cardiac problems. }\end{array}$ & 1.01 & 0.96 & 1.06 & \\
\hline Supporting cardiac patients to stop smoking is important & 1.38 & 1.26 & 1.47 & $<0.05$ \\
\hline $\begin{array}{l}\text { It is important that cardiology wards support cardiac patients } \\
\text { to stop smoking }\end{array}$ & 1.33 & 1.16 & 1.46 & $<0.01$ \\
\hline $\begin{array}{l}\text { Nurses play an important role in the decision of cardiac } \\
\text { patients to quit smoking }\end{array}$ & 0.66 & 0.47 & 0.83 & $<0.05$ \\
\hline It is important that cardiac patients quit smoking & 1.54 & 1.46 & 1.61 & \\
\hline Smoking cessation education is a task of cardiology nurses & 1.31 & 1.14 & 1.44 & $<0.01$ \\
\hline Cues to action: Dissatisfaction $(-2=$ disagree,$+2=$ agree $)$ & 0.52 & 0.47 & 0.57 & \\
\hline $\begin{array}{l}\text { Before the C-MIS was introduced, I thought my smoking } \\
\text { cessation support should be improved }\end{array}$ & 0.58 & 0.46 & 0.68 & $<0.05$ \\
\hline $\begin{array}{l}\text { Before the C-MIS was introduced, I was not satisfied with the } \\
\text { smoking cessation support I gave to patients }\end{array}$ & 0.47 & 0.48 & 0.46 & \\
\hline \multicolumn{5}{|l|}{ Risk perception } \\
\hline $\begin{array}{l}\text { Perceived risk of smoking in terms of worsening cardiac } \\
\text { disease }(1=\text { no risk, } 10=\text { very high risk })\end{array}$ & 8.86 & 8.73 & 8.98 & \\
\hline $\begin{array}{l}\text { Effect of smoking cessation on the risk of having another heart } \\
\text { attack }(-2=\text { decreases }>20 \%,+2=\text { increases }>20 \%)\end{array}$ & -1.49 & -1.52 & -1.46 & \\
\hline
\end{tabular}

$\$ p$-values for differences between means were tested with t-test, and are only presented if significant

The two groups did not differ significantly in terms of their perception of the risks of smoking, nor on the dissatisfaction scale. Yet, adherent users were significantly more likely to believe that their cessation support had been in need of improvement before the C-MIS was introduced. 
Table 3. Means of and significant differences in attitude items, for adherent and nonadherent users

\begin{tabular}{|c|c|c|c|c|}
\hline Variable (scale) & $\begin{array}{l}\text { Overall } \\
(\mathrm{N}=206)\end{array}$ & $\begin{array}{c}\text { Non- } \\
\text { adherent } \\
\text { users } \\
(n=94)\end{array}$ & $\begin{array}{c}\text { Adherent } \\
\text { users } \\
(n=112)\end{array}$ & $\begin{array}{c}\mathrm{p}- \\
\text { value }\end{array}$ \\
\hline \multicolumn{5}{|l|}{ Attitude items (-2=disagree, $+2=$ agree $)$} \\
\hline Pros & 0.58 & 0.37 & 0.74 & $<0.01$ \\
\hline $\begin{array}{l}\text { The C-MIS protocol is a useful tool to help patients think about the } \\
\text { pros of quitting }\end{array}$ & 1.13 & 1.00 & 1.25 & $<0.01$ \\
\hline The C-MIS protocol helps me to motivate patients to stop smoking & 0.83 & 0.62 & 1.01 & $<0.01$ \\
\hline The C-MIS protocol helps me to fulfil my preventive task & 0.88 & 0.70 & 1.03 & $<0.01$ \\
\hline $\begin{array}{l}\text { The C-MIS helps me to address patients' smoking behavior in a } \\
\text { systematic way }\end{array}$ & 0.91 & 0.67 & 1.12 & $<0.01$ \\
\hline $\begin{array}{l}\text { The C-MIS protocol is a good instrument to prevent patients from } \\
\text { relapse after leaving the hospital }\end{array}$ & 0.30 & 0.15 & 0.44 & $<0.05$ \\
\hline $\begin{array}{l}\text { The C-MIS helps me to address smoking in an easier and more } \\
\text { pleasant way }\end{array}$ & 0.70 & 0.48 & 0.88 & $<0.01$ \\
\hline $\begin{array}{l}\text { The C-MIS is more effective in getting patients to quit smoking } \\
\text { than normal smoking cessation care }\end{array}$ & 0.50 & 0.35 & 0.62 & $<0.01$ \\
\hline $\begin{array}{l}\text { The C-MIS is an improvement compared to our former smoking } \\
\text { cessation approach }\end{array}$ & 0.61 & 0.50 & 0.70 & $<0.05$ \\
\hline The C-MIS is flexible and easy to adapt to daily practice & 0.56 & 0.40 & 0.70 & $<0.01$ \\
\hline $\begin{array}{l}\text { I have a clear idea of the positive effect of the C-MIS on the } \\
\text { smoking behavior of my patients }\end{array}$ & 0.05 & -0.23 & 0.28 & $<0.01$ \\
\hline $\begin{array}{l}\text { I had the option to try the C-MIS before my definitive decision to } \\
\text { use it }\end{array}$ & -0.25 & -0.43 & -0.10 & $<0.05$ \\
\hline $\begin{array}{l}\text { The C-MIS makes it easier for me to help patients with smoking } \\
\text { cessation }\end{array}$ & 0.81 & 0.56 & 1.03 & $<0.01$ \\
\hline $\begin{array}{l}\text { The MIS protocol increases the quality of my education on } \\
\text { smoking cessation }\end{array}$ & 0.82 & 0.60 & 1.00 & $<0.01$ \\
\hline $\begin{array}{l}\text { The C-MIS helps more patients to stop smoking, than other forms } \\
\text { of education }\end{array}$ & 0.32 & 0.12 & 0.49 & $<0.01$ \\
\hline Cons & -0.89 & -0.73 & -1.01 & $<0.01$ \\
\hline The C-MIS protocol does not fit my way of working & -0.69 & -0.43 & -0.92 & $<0.01$ \\
\hline If I use the C-MIS protocol I bother my patients & -0.93 & -0.74 & -1.08 & $<0.01$ \\
\hline If I use the C-MIS I limit my freedom of working & -0.99 & -0.88 & -1.07 & $<0.05$ \\
\hline The C-MIS protocol is hard to understand & -0.84 & -0.65 & -1.00 & $<0.01$ \\
\hline Using the C-MIS has more disadvantages than advantages & -0.85 & -0.73 & -0.95 & $<0.01$ \\
\hline Using the C-MIS takes too much time & -0.65 & -0.40 & -0.88 & $<0.01$ \\
\hline The C-MIS is difficult to apply & -0.91 & -0.75 & -1.05 & $<0.01$ \\
\hline The MIS protocol is not feasible in daily practice & -0.89 & -0.76 & -0.99 & $<0.01$ \\
\hline Using the C-MIS is too much of a burden to me & -0.95 & -0.78 & -1.09 & $<0.01$ \\
\hline Use of the C-MIS harms the relation I have with my patients & -1.07 & -1.00 & -1.13 & \\
\hline Attitude ambivalence & -0.77 & -0.46 & -1.03 & $<0.01$ \\
\hline I am not sure about my opinion regarding the C-MIS & -0.67 & -0.35 & -0.94 & $<0.01$ \\
\hline I doubt whether I think that I should work with the C-MIS & -0.88 & -0.58 & -1.13 & $<0.01$ \\
\hline
\end{tabular}

$\$ p$-values for differences between means were tested with t-tests, and are only presented if significant

\section{Motivational Factors}

Tables 3 and 4 show that adherent users differed significantly from nonadherent users in terms of all motivational concepts. Differences and significance levels for each attitude item are shown in Table 3. In general, nurses perceived more pros than cons of the CMIS (0.58 vs. -0.89 agreement). 
Table 4. Means of and significant differences in social influence and self-efficacy items, for adherent and nonadherent users

\begin{tabular}{|c|c|c|c|c|}
\hline Variable (scale) & $\begin{array}{l}\text { Overall } \\
(\mathrm{N}=206)\end{array}$ & $\begin{array}{c}\text { Non- } \\
\text { adherent } \\
\text { users } \\
(n=94)\end{array}$ & $\begin{array}{l}\text { Adherent } \\
\text { users } \\
(n=112)\end{array}$ & $\begin{array}{c}\mathrm{p}- \\
\text { value }\end{array}$ \\
\hline \multicolumn{5}{|l|}{ Social influence items } \\
\hline Social norms $(-2=$ negative,$+2=$ positive norm $) ;$ scale $(-8,+8)$ & 5.23 & 4.71 & 5.68 & $<0.01$ \\
\hline Perceived norm from nurses & 1.42 & 1.28 & 1.53 & $<0.01$ \\
\hline Perceived norm from ward head & 1.56 & 1.42 & 1.68 & $<0.01$ \\
\hline Perceived norm from cardiologist & 1.42 & 1.29 & 1.52 & $<0.05$ \\
\hline Perceived norm from patients & 0.85 & 0.74 & 0.94 & \\
\hline Social support $(-2=$ discouragement,$+2=$ support $)$; scale $(-8,+8)$ & 3.54 & 3.12 & 3.91 & $<0.01$ \\
\hline Perceived support from nurses & 1.03 & 0.93 & 1.11 & $<0.05$ \\
\hline Perceived support from ward head & 1.09 & 0.93 & 1.23 & $<0.01$ \\
\hline Perceived support from cardiologist & 0.83 & 0.71 & 0.94 & $<0.05$ \\
\hline Perceived support from patients & 0.62 & 0.54 & 0.68 & \\
\hline \multicolumn{5}{|l|}{ Modeling } \\
\hline $\begin{array}{l}\text { Perceived percentage of all nurses on the respondents' own } \\
\text { wards using the C-MIS }\end{array}$ & 78 & 69 & 85 & $<0.01$ \\
\hline \multicolumn{5}{|l|}{ Self-efficacy items } \\
\hline \multicolumn{5}{|l|}{$\begin{array}{l}\text { Do you manage to use the C-MIS protocol to help patients quit } \\
\text { smoking, if } \ldots(-3=\text { definitely not },+3=\text { definitely })\end{array}$} \\
\hline Stress & -0.33 & -0.54 & -0.15 & $<0.01$ \\
\hline - it is busy on the ward & -0.52 & -0.96 & -0.15 & $<0.01$ \\
\hline - the ward is being reorganized & 0.11 & -0.15 & 0.33 & $<0.01$ \\
\hline - you are working with substitutes for ill colleagues & -0.54 & -0.49 & -0.57 & \\
\hline Situation: resistant patient & -1.36 & -1.52 & -1.23 & $<0.05$ \\
\hline - your patient is not interested & -1.44 & -1.58 & -1.33 & \\
\hline - tyour patient resists talking about smoking & -1.27 & -1.45 & -1.11 & $<0.05$ \\
\hline Situation: patient characteristics & -0.13 & -0.28 & 0.00 & \\
\hline - your patient has a low level of education & 0.42 & 0.22 & 0.59 & $<0.05$ \\
\hline - your patient has problems with the Dutch language & -0.69 & -0.79 & -0.60 & \\
\hline Routine & 0.94 & 0.83 & 1.03 & \\
\hline - you have not used the C-MIS for a while & 0.91 & 0.76 & 1.04 & \\
\hline - you have not used the C-MIS regularly in recent months & 0.98 & 0.82 & 1.12 & \\
\hline - you did not use the MIS for your previous smoking patients & 0.90 & 0.87 & 0.93 & \\
\hline Social & -0.35 & -0.40 & -0.31 & \\
\hline - you know your colleagues do not use the C-MIS & -0.26 & -0.27 & -0.25 & \\
\hline - use of the C-MIS is not coordinated by the ward & -0.44 & -0.54 & -0.36 & \\
\hline
\end{tabular}

$\$ p$-values for differences between means were tested with t-tests, and are only presented if significant

Adherent users had a significantly more positive attitude toward the C-MIS. They were more convinced of the positive innovation characteristics and of the positive outcomes of using the C-MIS and they perceived less cons of using the C-MIS. Adherent users were also significantly less ambivalent, which means that they had a stronger opinion on the C-MIS.

Table 4 shows that adherent users perceived more positive norms and more support from their social environment than nonadherent users (5.68 vs 4.71 and 3.91 vs. 3.12 respectively). Only the perceived norms and support from patients did not differ 
significantly between the two groups. Additionally, adherent users perceived a larger proportion of colleagues on their wards using the C-MIS than non-adherent users.

Adherent users had higher self-efficacy than nonadherent users in stressful situations, when a patient resisted talking about the C-MIS, and when a patient had a low educational level. Self-efficacy in the case of a lack of routine or a lack of supportive social environment did not differ between the two groups.

\section{Postmotivational Factors}

With respect to the ability factors, Table 5 shows that adherent users were significantly more convinced of their skills to implement the different steps of the C-MIS than nonadherent users. Their beliefs about skills to assess smoking behavior and patients' motivation to quit did not differ significantly, but their beliefs about skills to perform the remaining steps did. In general, the lowest perceived skills were reported for addressing the use of smoking cessation aids and providing follow-up care.

Table 5. Means of and significant differences in ability factors and barriers, for adherent and nonadherent users ${ }^{1}$

\begin{tabular}{|c|c|c|c|c|}
\hline Variable (scale) & $\begin{array}{l}\text { Overall } \\
(\mathrm{N}=206)\end{array}$ & $\begin{array}{c}\text { Non- } \\
\text { adherent } \\
\text { users } \\
(n=94)\end{array}$ & $\begin{array}{c}\text { Adherent } \\
\text { users } \\
(n=112)\end{array}$ & p-value $\$$ \\
\hline Skills $(-2=$ disagree,$+2=$ agree $)$ & 0.94 & 0.76 & 1.08 & $<0.01$ \\
\hline \multicolumn{5}{|l|}{ I have sufficient skills to... } \\
\hline -help patients with smoking cessation according to the C-MIS & 0.81 & 0.55 & 1.03 & $<0.01$ \\
\hline -ask a patients for his smoking behavior & 1.15 & 1.09 & 1.20 & \\
\hline -assess a patient's motivation to quit & 1.12 & 1.07 & 1.17 & \\
\hline -talk about the barriers to smoking cessation & 1.09 & 1.01 & 1.16 & $<0.05$ \\
\hline -address the use of smoking cessation aids & 0.83 & 0.60 & 1.02 & $<0.01$ \\
\hline -set a date for quitting & 1.02 & 0.86 & 1.15 & $<0.01$ \\
\hline $\begin{array}{l}\text {-contact a patient by phone after discharge to discuss } \\
\text { smoking }\end{array}$ & 0.56 & 0.22 & 0.84 & $<0.01$ \\
\hline \multicolumn{5}{|l|}{ Goal actions ( $0=$ never, $4=$ very often) } \\
\hline General goal actions & 1.71 & 1.49 & 1.89 & $<0.01$ \\
\hline I talk about the C-MIS with the cardiologist & 1.33 & 1.11 & 1.49 & $<0.05$ \\
\hline I talk about the C-MIS with colleagues & 1.76 & 1.48 & 1.95 & $<0.01$ \\
\hline $\begin{array}{l}\text { I ask the cardiologist if he has advised my patient to stop } \\
\text { smoking }\end{array}$ & 2.03 & 1.69 & 2.28 & $<0.01$ \\
\hline Situation specific goal actions & 1.61 & 1.48 & 1.72 & $<0.05$ \\
\hline $\begin{array}{l}\text { If I have problems applying the C-MIS to one of my patients, I } \\
\text { consult one of my colleagues }\end{array}$ & 1.45 & 1.21 & 1.62 & $<0.01$ \\
\hline Before seeing a patient, I think about how to apply the C-MIS & 1.55 & 1.43 & 1.64 & \\
\hline When a colleague takes over a patient, we discuss the C-MIS & 1.83 & 1.73 & 1.91 & \\
\hline Use of intervention card ( $0=$ never, $4=a /$ ways $)$ & 2.67 & 1.78 & 3.50 & $<0.01$ \\
\hline Barriers: Availability of materials (index $0-2)^{+}$ & 1.57 & 1.37 & 1.79 & $<0.01$ \\
\hline Patient information brochure (\% yes, always available) & 81.5 & 72.2 & 89.1 & $<0.01 *$ \\
\hline Intervention card (\% yes, always available) & 83.4 & 71.1 & 93.6 & $<0.01 *$ \\
\hline
\end{tabular}

${ }^{1}$ The value in the table is the mean value unless indicated otherwise ${ }^{\$} \mathrm{p}$-values for differences between means were tested with t-tests unless indicated otherwise, and are only presented if significant * Differences between the groups were tested with chi-square tests ${ }^{\dagger}$ sum of scores (yes $=1$ ) on availability of materials 
Adherent users of the C-MIS were more likely to carry out goal actions than nonadherent users. Especially the intervention card was much more consistently used by adherent users $(M=3.50)$ than by nonadherent users $(M=1.78)$. Although both significant, there was a bigger difference between adherent and nonadherent users in performing general goal actions, than in performing situation specific goal actions.

Regarding barriers Table 5 shows that adherent users were significantly less likely than nonadherent users to report that the necessary C-MIS materials (patient information brochures and intervention cards) were not always available.

\subsubsection{Level of Association}

Figure 2 presents the Pearson correlation coefficients of the variables with the strongest $(>0.20)$, significant correlations with adherence. Figure 2 shows, from the left side to the right, that adherence correlated most strongly with intervention card use, followed by perceiving more advantages, positive modeling, lower levels of ambivalence, perceiving fewer disadvantages, more skills, high availability of materials, high knowledge level, high involvement in decision making, high general goal actions, high personal relevance, positive social norms.

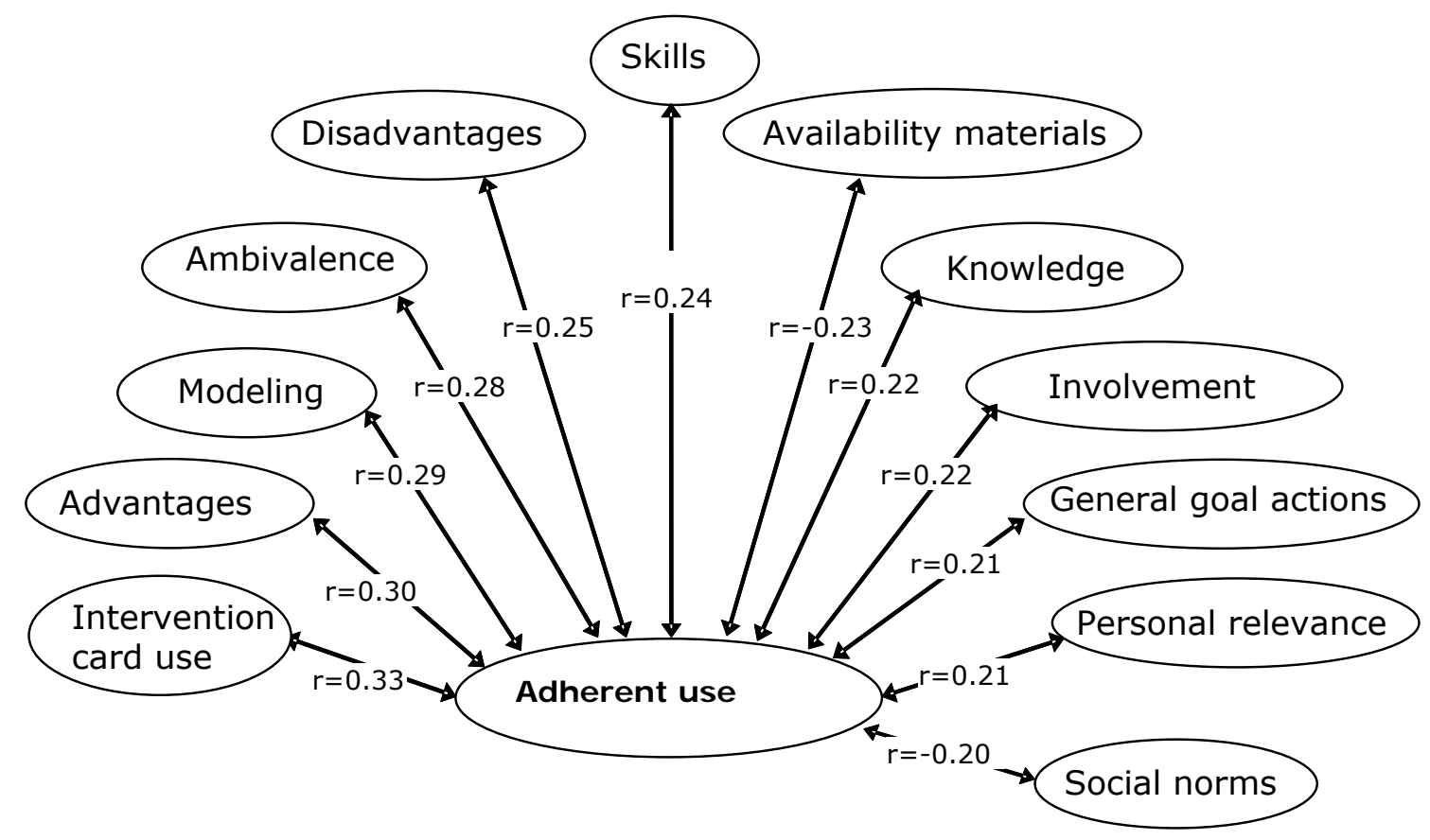

Figure 2. Pearson correlations of the most important factors associated with adherence

\subsubsection{Applicability of the I-Change Model}

Sequential regression analyses showed that the factors in the I-Change Model were associated with $52 \%$ of the variance between nurses in their adherence to the C-MIS 
protocol. Premotivational factors were associated with $26 \%$ of the variance in adherence. A summary of the results is presented in Table 6. Of the premotivational factors, having a room on the ward where nurses can smoke was the only variable that was significantly negatively associated with adherence $(\beta=-0.42, p<0.05)$ when the other premotivational factors were controlled for. Yet, although not significant at $p<0.05$, there were fairly high partial associations between adherence and involvement in decision-making ( $\beta=0.34$, $p<0.10)$, personal relevance $(\beta=0.33, p<0.10)$, and the amount of information usually collected before decisions were made $(\beta=0.28, p<0.10)$. Adding motivational factors to the model significantly increased $\left(X^{2}=31.1, p<0.001\right)$ the association with variance in adherence to $38 \%$. The association between premotivational factors and adherence was largely mediated by motivational factors. Only the negative association between adherence and having a designated room on the ward where nurses smoke was not mediated by motivational factors; in fact, it even strengthened $(\beta=-0.52, p<0.01)$. Perceived advantages $(\beta=0.56, p<0.05)$ and modeling $(\beta=0.51, p<0.05)$ were the only motivational variables that were highly associated with adherence when controlling for the other variables. Adding postmotivational factors significantly increased $\left(X^{2}=32.1\right.$, $\mathrm{p}<0.001)$ the association with the variance in adherence to $52 \%$. Postmotivational factors partly mediated the association between motivational factors and adherence. The only significant partial association was found between the use of the intervention card and adherence $(\beta=1.05, p<0.001)$.

\subsection{Discussion}

The first main objective of our study was to assess adherence to the C-MIS protocol by individual nurses and to identify which steps of the protocol were more difficult to apply than others. We found that almost half of the nurses did not adequately adhere to the CMIS in daily practice. This was in line with findings from a previous Dutch pilot study among nurses on cardiac wards that showed that after implementation of the C-MIS on the ward $49 \%$ of the nurses on those wards did not support the majority or all of their patients with the C-MIS (Bolman et al., 2002). Aspects that were particularly underapplied were: providing smoking patients with the self-help guide about smoking cessation, discussing smoking cessation aids, and providing follow-up care. Reasons for not providing patients with the self-help guide included that the guides were not always available on the ward and that nurses sometimes forgot to provide them. A reason for not discussing smoking cessation aids and providing follow-up care was that some nurses felt they lacked the necessary skills. This is indicated by our finding that these were the actions for which the lowest skills were reported. Remarkable is that selfreported skills for providing follow-up were low, since the C-MIS skills training paid special attention to this. 
The second main objective of the present study was to identify differences between adherent and nonadherent users of the C-MIS. Adherent and nonadherent users differed significantly in terms of three organizational characteristics. Adherent users were more likely to work on a ward without a designated room where nurses could smoke, collected more information before making decisions, and were more likely to be involved in decision-making. A smoking room on the ward might reduce the credibility of the smoking cessation message, because patients are confronted with nurses who smoke themselves. The difference in involvement is in line with the finding by Cooke and colleagues (2000) that involvement was a significant predictor of all stages of the diffusion process, and with the assumption of social marketing theories that participation in decision-making increases people's motivation to accept change (Hage \& Aiken, 1970; Kotler \& Roberto, 1991).

Adherent users were older and had more years of experience working as a nurse than nonadherent users. Work experience may have caused the association between age and adherence as there was no significant association between age and adherence when controlling for the number of years respondents had been working as a nurse. Although we found a fairly large difference (16 vs. $8 \%$ ) in smoking rates between nonadherent and adherent users, we did not find a significant effect of smoking habit on adherence $(p=0.06)$. The lack of finding a significant difference might be due to the relative small number of smokers in the study $(n=24)$. Therefore, the effect of nurses' smoking behavior on the provision of smoking cessation guidance remains unclear.

As regards motivational factors, adherent users had a more positive attitude towards using the C-MIS. They were more convinced of this positive attitude and they perceived more positive social influences. These findings are in line with those of other studies on the diffusion of health education programs (Bolman et al., 2002; Mesters \& Meertens, 1999), as well as with social-cognitive theories (Ajzen, 1991; Bandura, 1986). Adherent users had higher self-efficacy to perform the C-MIS in stressful situations and with resistant patients, but, in general, self-efficacy was quite low in both adherent and nonadherent users. This finding is in line with that of the study by Bolman and colleagues (2002), although it contradicts the pattern found in studies about the determinants of smoking cessation in various motivational stages (De Vries \& Backbier, 1994; De Vries \& Mudde, 1998). The study by De Vries \& Mudde (1998) showed that self-efficacy increases mainly during the later stages of smoking cessation. This pattern may not fit diffusion stages or protocol use or that adherent users may be stricter about what the use of the C-MIS protocol implies and, therefore, report low self-efficacy.

With respect to postmotivational factors, adherent users perceived greater skills, performed more goal actions, and were less likely to report that they lacked the necessary materials. These findings were all in line with our expectations. We found a 
difference between the various types of goal action in the size of their relation with adherence. Especially intervention card use was highly associated with adherence. Use of the intervention card may be an action that always guides nurses through the steps; it can be seen as a kind of checklist including all questions that need to be asked of the patient. Answers can be reported in the same form and it is aimed at facilitating full application of the C-MIS. The goal action of having conversations with people about the MIS is less predictive, constant, and complete than using the card.

As regards our secondary objectives (assessing the applicability of the I-Change model in implementation research on protocol use by nurses and identifying the level of association between adherence and the factors in the model), we found that all factors included in the I-Change Model were significantly associated with adherence to the CMIS. These factors together were associated with $52 \%$ of the variance in adherence between nurses, which is quite high compared to findings of other studies that applied social-cognitive models to explain and predict health-related behaviors (Godin \& Kok, 1996). We also found that the relationship between premotivational factors and adherence was mediated by the motivational factors. Postmotivational factors partly mediated the association of motivational factors with adherence. Both mediations were in line with the assumptions of the I-Change Model.

Highest associations with adherence were found for a postmotivational factor, namely, the goal action intervention card use. This high association persisted even when controlling for the influence of all other factors that were significantly associated with adherent use, such as the motivational factors attitude and modeling. This finding is an indication of the importance of postmotivational factors in adherent protocol use. As there is no consistent view of what role postmotivational factors actually play in determining behavior, we recommend more research on these factors (e.g., to identify the underlying constructs that explain why the use of an intervention card is so highly associated with adherence). Apart from this, unreasoned behavior processes, such as habits and automaticity, might play a role.

Our study had some limitations. First, the study yielded only cross-sectional data, precluding causal inferences. Second, the participation rate was only $38 \%$. Despite our efforts to stimulate ward heads to have nurses complete the questionnaire, we cannot exclude the possibility of selection bias. This may have caused us to overrate adherence to the C-MIS. Yet, there was no significant difference between the reported proportion of colleagues who used the C-MIS and the proportion of nurses who reported using the CMIS themselves. Third, despite our preventive efforts, nurses may have provided biased (socially desirable) reports, which may also have resulted in overrating of the adherence to the C-MIS. Lastly, some of the subscales such as dissatisfaction and attitude 
ambivalence consisted of only 2 items. Therefore the reliability and validity of these scales is limited.

In conclusion, despite some limitations, the study has provided important information on nurses' adherence to a smoking cessation protocol, as well as on factors associated with the adherence to protocols for smoking cessation guidance in hospital wards and on the aspects of smoking cessation assistance that nurses are most likely to fail to accomplish. The I-Change Model proved to be a useful tool to identify potential determinants of adherence to health promotion interventions in hospitals. We recommend that future researchers examine whether the model as used in our study is valid in a longitudinal design and whether more exploration on the post-motivational factors would be valuable.

Finally, recommendations for practice are that nurses should further improve their follow-up smoking cessation care for patients, as well as the discussion of smoking cessation aids and the provision of self-help materials to patients. Nurses' skills have to be improved, e.g. by providing them with an improved training on counseling techniques, especially on follow-up counseling by phone, and providing them with more information on smoking cessation aids for cardiac patients. A reminder system should be developed to ensure that the self-help guides for patients are ordered in time. When future smoking cessation programs are to be implemented, all nurses on the ward should be involved in the decision-making process. Circumstances on the ward, such as a room where patients see nurses smoke that might impede the use of the protocol, should be banned. Furthermore, strategies should be developed to increase positive modeling effects by increasing the numbers of nurses on the ward who are perceived to use the intervention. For example, this could be achieved by promoting peer contacts on the subject and by presenting good practices and role models. Strategies should also aim to increase levels of perceived advantages of using the protocol. As soon as improved perceptions increase the number of nurses who use the C-MIS, this will in turn strengthen the modeling effect. A final recommendation is to intensively promote the consistent use of intervention cards as they serve as a registration system, as a schematic summary of the protocol, and as a reminder to perform all steps of the MIS, including handing out self-help guides to patients. 



\section{CHAPTER 4}

Identifying determinants of protocol adoption by midwives: A comprehensive approach

Not everything that counts can be counted, and not everything that can be counted counts. 
Abstract

Adoption of potentially effective preventive interventions often fails. This study aimed to identify factors that determine why midwifery practices decide to use a smoking cessation protocol, using a comprehensive model of both organizational and psychosocial factors. A cross-sectional survey was conducted among representatives of all 446 Dutch midwifery practices, of whom 251 (56\%) responded. The results show that adoption of the protocol was facilitated by the presence of practice assistants and impeded by a large proportion of clients of foreign ethnic origin. The most successful information channel was the midwives' professional association. A consistent positive attitude (perceiving a lot of advantages, few disadvantages and a low level of ambivalence) and positive social norms toward using the protocol, a perceived large proportion of midwives who use the protocol and knowledge about the protocol significantly increased the likelihood of adoption. The decision to use the protocol was better explained by personal awareness and motivation factors than by organizational factors.

\section{Slightly different version published as:}

Segaar, D., Bolman, C., Willemsen, M.C., De Vries, H. (in press). Identifying determinants of protocol adoption by midwives: A comprehensive approach. Health Education Research 


\subsection{I ntroduction}

Smoking during pregnancy causes several risks to the unborn, such as low birth weight and fetal death (US Department of Health and Human Services, 2001; British Medical Association, 2004). Smoking cessation is important to reduce negative pregnancy outcomes. Smoking cessation programs provided during pregnancy have proved to be efficacious in reducing smoking among pregnant women (TNO Preventie en Gezondheid, 2000; Lumley, Oliver, Chamberlain, \& Oakley, 2004). In the Netherlands, midwives assist about 75\% of all pregnant women (TNO Preventie en Gezondheid, 2000), and for most women, midwives are the main source of information on pregnancy related subjects. Women visit their midwives at least once a month during a regular pregnancy. Thus, midwives are regarded as ideal intermediaries to provide pregnant women with smoking cessation assistance. More than $90 \%$ of all Dutch midwives work in group practices of about three midwives. About half of them have practice assistants who can take over some tasks (Kenens \& Hingstman, 2004).

Till recently, midwives in the Netherlands usually did not pay systematic attention to smoking. Most midwives discussed smoking with their clients, but only to a limited extent (NIPO, 2002). Midwives indicated that they were motivated to provide smoking cessation counseling, but they did not feel sufficiently capable to provide the counseling (Bakker, De Vries, Mullen, \& Kok, 2005). For this reason an effective smoking cessation and relapse prevention protocol for midwives has been developed in the Netherlands (Bakker, Mullen, De Vries, \& Van Breukelen, 2003). The protocol is called the Minimal Intervention strategy for Smoking cessation (MIS) in midwifery practices. It is a stepwise strategy that focuses on the motivational level of pregnant women. It is comparable to the Ask, Advice, Assess, Assist and Arrange (5 A's) model, which is recommended in Smoking Cessation Guidelines for Health Professionals (Raw, McNeill, \& West, 1998; Van Weel et al., 2005).

The Dutch Expert Center on Tobacco Control (STIVORO) has distributed the protocol among midwifery practices in the Netherlands in cooperation with the Royal Dutch Organization for Midwives through various channels, such as direct mailing, professional journals, and conferences. Training courses on how to use the MIS have been offered, and relevant materials, such as manuals and an information magazine for pregnant women, have been provided as well. Since the adoption of potentially effective preventive interventions in the target field often fails (Haines \& Donald, 1998), the question arises whether midwives actually do adopt the protocol, and why.

Adoption has been studied by various disciplines, ranging from business marketing to psychology. These studies have identified a wide variety of factors influencing adoption, such as organization size (Castle, 2001; Moch \& Morse, 1977), group 
membership (Castle, 2001), and innovation characteristics (Rogers, 1995). However, these results are difficult to extrapolate to other settings because context-specific factors appear to be involved (Foy, Eccles, \& Grimshaw, 2001; Glasgow, Lichtenstein, \& Marcus, 2003). Consequently, research is needed to identify reasons for adoption in specific settings and specific populations.

The aim of our study was to identify organizational characteristics and personal (psychological) characteristics of the decision maker that determine the adoption of health education innovations by midwifery practices. More specifically, the study focused on the adoption of the MIS. We aimed to identify the factors that had the strongest associations with adoption of this specific intervention and studied differences between three adoption groups: non-intenders, intenders, and users.

\subsubsection{Theoretical model}

To assess the determinants of adoption of the MIS by midwifery practices, the present study used the Integrated Change (I-Change) Model (De Vries, Mesters, Van de Steeg, \& Honing, 2005; De Vries, Mudde, Leijs et al., 2003; De Vries, Mesters, Van 't Riet, Willems, \& Reubsaet, 2006), which integrates concepts of various social-cognitive models (Ajzen, 1991; Bandura, 1986; Prochaska \& DiClemente, 1983; Weinstein, 1988). The model is presented in Figure 1.

The I-Change Model states that behavior is determined by intention. Transition from the intention state to the behavioral state is determined to an important extent by the ability to perform the behavior and by barriers. Since adoption takes place at the intention stage, the post motivation factors in the I-Change Model, i.e. ability factors and barriers, were not examined in the current study. Intention state is determined by three types of proximal motivation factors; Attitude, Social Influence, and Self-Efficacy. Attitude includes the beliefs about the innovation characteristics defined by Rogers (Rogers, 2002), namely perceived relative advantage, compatibility, complexity, trialability and visible results. These factors have already been used to explain the adoption of various types of health behavior, like classroom-based education (Paulussen, Kok, \& Schaalma, 1994), use of hormone replacement therapy (Wilhelm, 2002), and physical activity behavior (Fahrenwald \& Walker, 2003).

The I-Change Model assumes that the proximal motivation factors are determined by various more distal (premotivation) factors, such as awareness factors (i.e., knowledge, cues to action, and risk perceptions), information factors (the quality of messages, channels, and sources used), and predisposing factors (i.e., behavioral factors, psychological factors, biological factors, and social and cultural factors). To identify relevant social and cultural factors, we used a contingency model of strategic decision making from the organizational psychology perspective, developed by Koopman 
and Pool (Koopman \& Pool, 1991;1994). The applicability of this model in implementation research has been described by Willemsen and colleagues (Willemsen, Meijer, \& Jannink, 1999). The model defines three major groups of characteristics influencing the decisionmaking process: the content of decision making, its context, and the decision-making style that is dominant in the organization. The context of decision making consists of characteristics of the environment, of the organization, and of the decision maker. Characteristics of the decision maker belong to the behavioral and biological factors in the I-Change model.

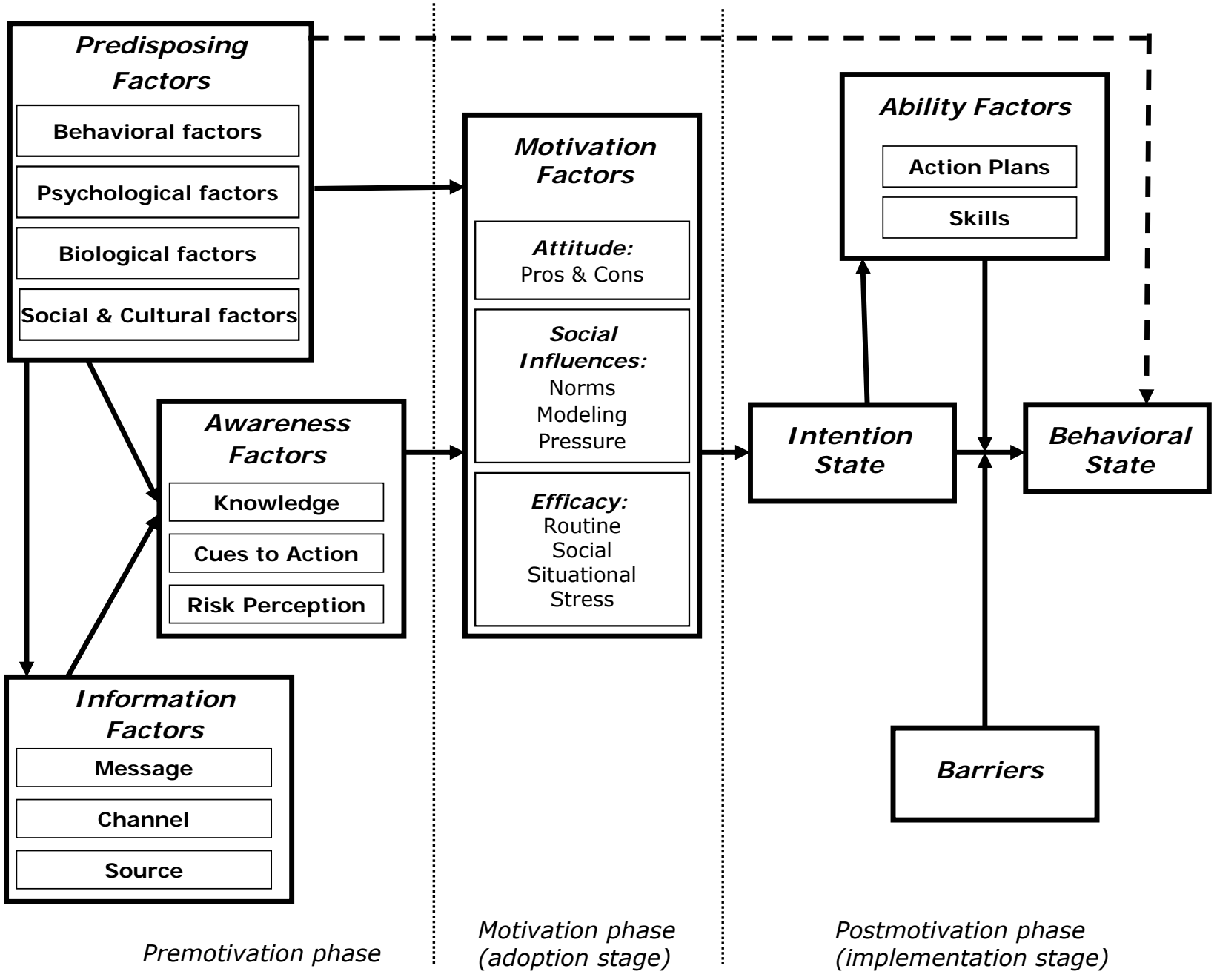

Figure 1. The I-Change Model (De Vries et al., 2005, 2006; De Vries, Mudde, Leijs et al., 2003) applied to the adoption of preventive interventions by midwifery practices.

\subsection{Methods}

\subsubsection{Study design and procedure}

A cross-sectional nationwide study was conducted in 2003; two years after the nationwide introduction of the MIS in the Netherlands had started. The study focused on all midwifery practices in the Netherlands $(\mathrm{N}=446)$. One written questionnaires was mailed to each practice, with the request to have it filled out by one of the midwives officiating as a representative of the practice. A letter explaining the aim of the study and 
a postage-paid return envelope were enclosed. To stimulate practices to return the questionnaire, the support of the national organization for midwives and the value of the opinion (maybe even more if negative) of each practice were stressed. As a reward respondents could win a $€ 25$ value coupon if they returned the questionnaire. Reminders were sent to the non-responders three weeks later.

\subsubsection{Questionnaire}

The questionnaire was based on existing questionnaires or relevant scales, and on information from literature reviews and earlier work (Bakker et al., 2003; Bolman, De Vries, \& Mesters, 2002; Mesters \& Meertens, 1999; Steckler, Goodman, McLeroy, Davis, \& Koch, 1992).

\section{Outcome variable}

To be able to categorize respondents in terms of their adoption state, three questions were asked. First, midwives were asked if they were already using the MIS in their practice (yes/no). If the answer was "no", a second question was whether they had made a conscious decision about using the MIS at all (yes/no), followed by a third question: "Do you intend to use the MIS in the future and if so when?" (i.e., in the next month, in the next 6 months, in the next year, ever but not within a year, or never).

Respondents were categorized into three groups according to their adoption state. The first group consisted of "non-intenders". These were respondents who were not using the MIS and were not seriously planning to start using it in the near future. Near future was defined as six months from the time of measurement. The second group was that of "intenders", respondents who were not using the MIS yet, but were seriously planning to use it in the near future. The third category was "users", respondents who were already using the MIS protocol at their practice.

\section{Predisposing factors}

Detailed information on the questions is provided in Table 1. Biological factors measured were the age and gender of the midwives. Behavioral factors assessed were the smoking behavior of the midwives and the number of years they had been working in their profession. Social and cultural factors were assessed by questions relating to the environmental and organizational context and the style of decision making. Environmental context characteristics included the proportion of clients of foreign ethnic origin and the proportion of clients with a low educational level. Measurements of context variables at the organizational level included six items, i.e., the number of midwives working in the practice, whether there were practice assistants (yes/no), the number of pregnancies monitored per year, the time available for the first visit (minutes), workload, 
and the innovativeness of the midwifery practice. Style of decision making was measured by five items: centralization (decisions entirely made by the individual midwives or centralized at practice level); formalization of decision making in the practice (own solutions or set procedures); the amount of information gathered preceding decisions; the proportion of external information sources versus internal information sources consulted for decisions and the way disagreements are solved.

\section{Information factors}

Detailed information on the questions about information factors is provided in Table 1. For the five most important information sources on the MIS midwives were asked if they consulted them.

\section{Awareness factors}

Detailed information on the questions about awareness is provided by Table 2 . Awareness knowledge was measured by four questions: "Have you ever heard of the MIS?", "Do you know what the MIS is?", "Do you know how the MIS works" and "Can you overlook the implications of using the MIS?". An index score was calculated by summing the number of times a midwife answered yes to these questions. Risk perception was assessed by asking two questions about perceived risks of smoking for the baby. A risk perception scale $(r=0.59)$ was calculated by averaging the scores of the two items. Cues to action were measured by one scale: personal relevance. Personal relevance was measured with four items and a personal relevance subscale (Cronbach's $a=0.70$ ) was calculated by averaging the item scores.

\section{Motivation factors}

Detailed information on the questions on motivation factors is provided by Tables 3, 4 and 5. Attitude (Table 3) was assessed by three constructs: pros, cons, and attitudinal ambivalence (Ajzen, 2001). Pros and cons about using the MIS were assessed by 25 questions, including 11 items on beliefs about the innovation characteristics (Bolman et al., 2002; Mesters \& Meertens, 1999; Pieterse, Seydel, De Vries, Mudde, \& Kok, 2001; Rogers, 2002; Steckler et al., 1992) and 14 items on perceived health benefits and other outcome expectations (Bandura, 1986) of using the MIS protocol (Bakker et al., 2003). A factor analysis with oblimin rotation on the attitude variables revealed two attitude factors: the pros ( 15 items, $a=0.90$ ) and the cons (10 items, $a=0.79$ ) of using the MIS protocol. Attitude ambivalence was measured by two questions, one scale $(r=0.73)$ was calculated by averaging the two item scores.

Perceived social influences (Table 4) were assessed using three constructs: perceived social support, perceived social norms, and social modeling. Perceived support 
and norms were assessed for six subgroups (see Table 4). For each subgroup, support was measured by asking "Do you perceive discouragement or support to work with the MIS?" and social norms were measured by the question "Do you perceive positive norms towards your assisting pregnant women with smoking cessation according to the MIS?". The scores for the subgroups were averaged to calculate one scale for support $(a=0.86)$ and one scale for norms $(a=0.88)$. Social modeling was assessed by one question asking the respondent to estimate the percentage of other Dutch midwives in primary health care who use the MIS.

Self-efficacy expectations (Table 5) were measured by asking midwives whether they would succeed in providing smoking cessation assistance with the MIS in 15 situations occurring in daily practice. Situations were derived from previous research on the adoption of smoking cessation programs in health care (Bakker et al., 2003; Cooke, Mattick, \& Campbell, 2000). A self-efficacy scale ( $a=0.87)$ was calculated by averaging the scores for the 15 situations.

\subsubsection{Statistical analyses}

Univariate analyses of variance (ANOVAs) using the Tukey post-hoc HSD contrasts (Stoline, 1981) were used to test differences between non-intenders, intenders, and users. Chi-square tests were used for dichotomous variables. The awareness and motivational variables were expected to change upon using the MIS. However, both intenders and users significantly differed from non-intenders on the scale scores for those variables. Hence, we combined intenders and users into one 'adopter' group to be able to identify the factors with the strongest association with adoption by performing logistic analyses with adoption as the dependent variable.

Three one-step logistic regression analyses were performed, using backward likelihood ratio procedures. In this type of analysis all variables are initially included in the regression equotation, then the variables that are judged by the model to be least important (based on the model's change in -2 log likelihood when removing the variable) are progressively removed from the model. This procedures continues as long as the removals do not significantly affect the fit of the model. The first analysis included all predisposing and information factors, in order to assess their relevance for adoption of the MIS. The second analysis added awareness factors in order to assess their additive contribution. In the third analysis, motivation factors were added, to identify their contribution and to identify which factors were strongest associated with adoption. Nagelkerke's $\mathrm{R}^{2}$ was used to approximate explained variances of the models (Nagelkerke, 1991). Assumptions for regression analysis and a check on multicollinearity between the independent variables were found to be satisfactory. All analyses were performed using SPSS version 12.0.1. Differences were considered significant when $p<0.05$. 


\subsection{Results}

\subsubsection{Description of sample}

A total of 251 of the 446 practice representatives (56\%) returned the questionnaire. Ninety-one respondents (36\%) used the MIS (users), 42 respondents (17\%) were planning to use the MIS (intenders), and 118 (47\%) were not planning to use the MIS (non-intenders). Of the responding midwives, 98\% were female; the mean age was 37 $(S D=10)$ years. Eight percent $(n=21)$ of the respondents smoked, and respondents had been working as a midwife for an average of $11(S D=9)$ years.

Table 1. Means of predisposing factors and information factors for non-intending, intending and using practices ${ }^{1}$, and significant differences

\begin{tabular}{|c|c|c|c|c|c|}
\hline Variable (scale) & $\begin{array}{c}\text { Overall } \\
(\mathrm{N}=251)\end{array}$ & $\begin{array}{c}\text { Non- } \\
\text { intenders } \\
(n=118)\end{array}$ & $\begin{array}{l}\text { Intenders } \\
(n=42)\end{array}$ & $\begin{array}{l}\text { Users } \\
(n=91)\end{array}$ & $\begin{array}{l}\text { Tukey } \\
\text { HSD* }\end{array}$ \\
\hline \multicolumn{6}{|l|}{ Biological factors } \\
\hline Percentage female (\% yes) & 98 & 97 & 100 & 98 & $*$ \\
\hline Age in years & 37 & 35 & 36 & 38 & \\
\hline \multicolumn{6}{|l|}{ Behavioral factors } \\
\hline Percentage of smokers (\% yes) & 8 & 7 & 7 & 11 & $*$ \\
\hline Years working as a midwife & 11 & 10 & 11 & 12 & \\
\hline \multicolumn{6}{|l|}{ Social and cultural factors } \\
\hline \multicolumn{6}{|l|}{ Environment } \\
\hline Estimated percentage of clients of foreign ethnic origin & 18 & 20 & 13 & 18 & $\mathrm{I}<\mathrm{N}$ \\
\hline Estimated percentage of clients with low education & 42 & 42 & 38 & 43 & \\
\hline \multicolumn{6}{|l|}{ Organization } \\
\hline Number of midwives in the practice & 3.4 & 3.3 & 3.3 & 3.6 & $\mathrm{~N}<\mathrm{U}, \mathrm{I}$ \\
\hline Percentage of practices with assistants (\% yes) & 50 & 41 & 62 & 57 & $*$ \\
\hline Number of pregnancies monitored per year & 331 & 339 & 285 & 343 & \\
\hline Time available for the first visit, in minutes & 34 & 33 & 33 & 35 & \\
\hline $\begin{array}{l}\text { Practices' level of innovativeness (change: }-2=\text { negative, } \\
+2=\text { positive) }\end{array}$ & 1.04 & 0.98 & 1.17 & 1.04 & \\
\hline Workload $(-3=$ too low,$+3=$ too high $)$ & 0.97 & 1.11 & 0.90 & 0.83 & \\
\hline \multicolumn{6}{|l|}{ Decision-making styles $(0=l o w, 4=h i g h)$} \\
\hline Centralization level & 2.83 & 2.71 & 2.83 & 2.97 & \\
\hline Formalization level & 2.26 & 2.12 & 2.32 & 2.42 & $\mathrm{~N}<\mathrm{U}$ \\
\hline Level of disagreement & 0.30 & 0.34 & 0.19 & 0.31 & \\
\hline $\begin{array}{l}\text { If disagreement occurs, it is solved through convincing by } \\
\text { discussion (\% yes) }\end{array}$ & 36 & 39 & 33 & 34 & $*$ \\
\hline Amount of information used & 3.75 & 3.83 & 3.71 & 3.68 & \\
\hline $\begin{array}{l}\text { Number of external information sources versus internal } \\
\text { sources }\end{array}$ & 2.03 & 2.09 & 2.05 & 1.96 & \\
\hline \multicolumn{6}{|l|}{ Information factors (\% received information from) } \\
\hline Royal Dutch Organization for Midwives & 60.2 & 50.8 & 59.5 & 72.5 & $\mathrm{~N}<\mathrm{U} *$ \\
\hline Dutch expert center on Tobacco Control (STIVORO) & 39.8 & 45.8 & 45.2 & 29.7 & $\mathrm{~N}>\mathrm{U} *$ \\
\hline National journal for midwives & 41.4 & 39.8 & 35.7 & 46.2 & \\
\hline Colleagues & 17.2 & 14.4 & 26.2 & 17.6 & \\
\hline Midwifery schools & 6.8 & 8.5 & 4.8 & 5.5 & \\
\hline
\end{tabular}

$\mathrm{U}=$ users, $\mathrm{I}=$ intenders, $\mathrm{N}=$ non-intenders, * Differences between the three groups tested with chi-square tests

${ }^{1}$ The value in table is the mean value, unless indicated otherwise 
4.3.2 Differences between practices not intending to use, intending to use and using MIS The mean scores of non-intenders, intenders, and users on the variables assessed are shown in Tables 1 to 5 .

\section{Predisposing factors}

Table 1 shows that users, intenders, and non-intenders only differed on a few predisposing factors. Intenders gave a significantly lower estimate of the proportion of clients of foreign ethnic origin in their practice than non-intenders. Users and intenders were significantly more likely to have practice assistants than non-intenders. Users were significantly more likely to work in highly formalized settings than non-intenders.

\section{Information factors}

Table 1 shows that users were significantly more likely than non-intenders to have received information from the Royal Dutch Organization for Midwives and less likely to have received it from the Dutch expert center on Tobacco Control.

\section{Awareness factors}

Table 2 reveals that there were many significant differences between the three groups in terms of awareness factors. Both intenders and users had significantly more knowledge about the MIS than non-intenders. Users also had significantly more knowledge than intenders. Both users and intenders found the provision of smoking cessation assistance for pregnant women by midwives significantly more important than non-intenders. Users and intenders did not differ significantly from each other. Users perceived significantly greater risks of smoking for the unborn than non-intenders.

Table 2. Means of and significant differences in awareness factors for non-intending, intending and using practices

\begin{tabular}{|c|c|c|c|c|c|}
\hline Variable (scale) & $\begin{array}{l}\text { Overall } \\
(\mathrm{N}=251)\end{array}$ & $\begin{array}{c}\text { Non- } \\
\text { intenders } \\
(\mathrm{n}=118)\end{array}$ & $\begin{array}{l}\text { Intenders } \\
(n=42)\end{array}$ & $\begin{array}{l}\text { Users } \\
(n=91)\end{array}$ & $\begin{array}{l}\text { Tukey } \\
\text { HSD }\end{array}$ \\
\hline Knowledge level $(0=$ not at all, $4=$ very well) & 3.03 & 2.38 & 2.98 & 3.90 & $\mathbf{N}<\mathbf{U}, \mathbf{I} ; \mathbf{I}<\mathbf{U}$ \\
\hline $\begin{array}{l}\text { Cue to action: Personal relevance } \\
(-2=\text { disagree }, 2=\text { agree })\end{array}$ & 1.09 & 0.82 & 1.46 & 1.26 & $\mathbf{N}<\mathbf{U}, \mathbf{I}$ \\
\hline $\begin{array}{l}\text { Assisting pregnant women with smoking cessation is } \\
\text { important }\end{array}$ & 1.38 & 1.18 & 1.69 & 1.51 & $\mathrm{~N}<\mathrm{U}, \mathrm{I}$ \\
\hline $\begin{array}{l}\text { Assisting pregnant women with smoking cessation by } \\
\text { midwife practices is important }\end{array}$ & 0.60 & 0.07 & 1.29 & 0.95 & $\mathrm{~N}<\mathrm{U}, \mathrm{I}$ \\
\hline $\begin{array}{l}\text { Midwives play an important role in the decision of } \\
\text { pregnant women to quit smoking }\end{array}$ & 0.54 & 0.24 & 0.98 & 0.73 & $\mathrm{~N}<\mathrm{U}, \mathrm{I}$ \\
\hline It is important that pregnant women quit smoking & 1.84 & 1.81 & 1.88 & 1.86 & \\
\hline Risk perception ( $1=$ no risk, $10=$ very high risk) & 8.72 & 8.55 & 8.90 & 8.84 & \\
\hline Perceived risk of smoking for the unborn & 8.84 & 8.62 & 9.00 & 9.04 & $\mathrm{~N}<\mathrm{U}$ \\
\hline Risk of smoking to baby's health after birth & 8.60 & 8.47 & 8.81 & 8.66 & \\
\hline
\end{tabular}

$\mathrm{U}=$ users, $\mathrm{I}=$ intenders, $\mathrm{N}=$ non-intenders 


\section{Motivation factors}

Tables 3 and 4 show that non-intenders differed significantly from both intenders and users in terms of all motivation concepts. Intenders and users did not differ significantly from each other on most motivation concepts, except modeling, one advantage subscale, and a few self-efficacy items.

Table 3. Means of and significant differences in attitude items for non-intending, intending and using practices

\begin{tabular}{|c|c|c|c|c|c|}
\hline Variable (scale) & $\begin{array}{c}\text { Overall } \\
(\mathrm{N}=251)\end{array}$ & $\begin{array}{c}\text { Non- } \\
\text { intenders } \\
(\mathrm{n}=118)\end{array}$ & $\begin{array}{l}\text { Intenders } \\
\quad(n=42)\end{array}$ & $\begin{array}{l}\text { Users } \\
(n=91)\end{array}$ & $\begin{array}{l}\text { Tukey } \\
\text { HSD }\end{array}$ \\
\hline Advantages scale $(-2=$ disagree,$+2=$ agree $)$ & 0.45 & 0.18 & 0.75 & 0.60 & $\mathbf{N}<\mathbf{U}, \mathbf{I}$ \\
\hline \multicolumn{6}{|l|}{ If I give smoking cessation education according to the MIS... } \\
\hline - the risk that small children become ill decreases & 1.03 & 0.79 & 1.33 & 1.20 & $\mathrm{~N}<\mathrm{U}, \mathrm{I}$ \\
\hline - I help women to quit smoking & 0.87 & 0.70 & 1.10 & 0.97 & $\mathrm{~N}<\mathrm{U}, \mathrm{I}$ \\
\hline - I improve in terms of fulfilling the task I have as a midwife & 0.30 & -0.17 & 0.85 & 0.63 & $\mathrm{~N}<\mathrm{U}, \mathrm{I}$ \\
\hline - I fulfill an important preventive task & 0.88 & 0.56 & 1.20 & 1.12 & $\mathrm{~N}<\mathrm{U}, \mathrm{I}$ \\
\hline - I show that I care about the health of my clients & 0.79 & 0.40 & 1.13 & 1.10 & $\mathrm{~N}<\mathrm{U}, \mathrm{I}$ \\
\hline - I prevent my clients from relapsing after they quit & -0.05 & -0.12 & 0.25 & -0.11 & $\mathrm{~N}, \mathrm{U}<\mathrm{I}$ \\
\hline - the proportion of patients who quit successfully increases & 0.33 & 0.17 & 0.55 & 0.43 & $\mathrm{~N}<\mathrm{U}, \mathrm{I}$ \\
\hline - I'm a better midwife & -0.26 & -0.61 & 0.28 & -0.09 & $\mathrm{~N}<\mathrm{U}, \mathrm{I}$ \\
\hline $\begin{array}{l}\text { Using the MIS increases the number of women who quit } \\
\text { smoking compared to our usual education }\end{array}$ & 0.31 & 0.11 & 0.57 & 0.42 & $\mathrm{~N}<\mathrm{U}, \mathrm{I}$ \\
\hline $\begin{array}{l}\text { Use of the MIS can easily be stopped if it turns out not to } \\
\text { work }\end{array}$ & 0.67 & 0.50 & 0.68 & 0.87 & $\mathrm{~N}<\mathrm{U}$ \\
\hline $\begin{array}{l}\text { The MIS protocol increases the quality of our smoking } \\
\text { cessation education }\end{array}$ & 0.73 & 0.40 & 1.05 & 0.94 & $\mathrm{~N}<\mathrm{U}, \mathrm{I}$ \\
\hline $\begin{array}{l}\text { The MIS protocol is more effective in getting pregnant } \\
\text { women to quit smoking than our former/current practice }\end{array}$ & 0.55 & 0.25 & 0.86 & 0.75 & $\mathrm{~N}<\mathrm{U}, \mathrm{I}$ \\
\hline Use of the MIS has a visible impact on pregnant women & 0.06 & 0.01 & 0.19 & 0.06 & \\
\hline $\begin{array}{l}\text { The impact on midwives of introducing the MIS in a } \\
\text { midwives' practice is easy to assess }\end{array}$ & 0.02 & -0.10 & 0.22 & 0.06 & $\mathrm{~N}<\mathrm{I}$ \\
\hline Using the MIS protocol in my practice is a positive thing & 0.57 & 0.10 & 1.03 & 0.9 & $\mathrm{~N}<\mathrm{U}, \mathrm{I}$ \\
\hline Disadvantages scale $(-2=$ disagree,$+2=$ agree $)$ & -0.22 & 0.04 & -0.42 & -0.43 & $\mathbf{N}>\mathbf{U}, \mathbf{I}$ \\
\hline \multicolumn{6}{|l|}{ If I give smoking cessation education according to the MIS .... } \\
\hline - my clients will experience this as a privacy invasion & -0.51 & -0.29 & -0.78 & -0.67 & $\mathrm{~N}>\mathrm{U}, \mathrm{I}$ \\
\hline - it will be too much of a burden to me & 0.34 & 0.67 & 0.08 & 0.06 & $\mathrm{~N}>\mathrm{U}, \mathrm{I}$ \\
\hline - It will limit my freedom of working & -0.64 & -0.39 & -0.75 & -0.90 & $\mathrm{~N}>\mathrm{U}, \mathrm{I}$ \\
\hline - it will take me a lot of time & 0.16 & 0.55 & -0.23 & -0.13 & $\mathrm{~N}>\mathrm{U}, \mathrm{I}$ \\
\hline - my daily routine will be upset & -0.28 & -0.07 & -0.53 & -0.42 & $\mathrm{~N}>\mathrm{U}, \mathrm{I}$ \\
\hline The MIS protocol is not feasible in daily practice & -0.11 & 0.20 & -0.22 & -0.43 & $\mathrm{~N}>\mathrm{U}, \mathrm{I}$ \\
\hline $\begin{array}{l}\text { The MIS protocol is not flexible and easy to adjust to daily } \\
\text { practice }\end{array}$ & -0.13 & 0.17 & -0.30 & -0.39 & $\mathrm{~N}>\mathrm{U}, \mathrm{I}$ \\
\hline The MIS protocol is hard to understand & -0.58 & -0.35 & -0.73 & -0.76 & $\mathrm{~N}>\mathrm{U}, \mathrm{I}$ \\
\hline The MIS is difficult to apply & -0.32 & -0.06 & -0.59 & -0.49 & $\mathrm{~N}>\mathrm{U}, \mathrm{I}$ \\
\hline A lot of training is necessary to use the MIS protocol & -0.04 & 0.09 & -0.19 & -0.12 & \\
\hline Ambivalence $(-2=$ disagree,$+2=$ agree $)$ & -0.40 & 0.19 & -0.94 & -0.93 & $\mathbf{N}>\mathbf{U}, \mathbf{I}$ \\
\hline I am not sure about my opinion regarding the MIS & -0.40 & 0.11 & -0.85 & -0.85 & $\mathrm{~N}>\mathrm{U}, \mathrm{I}$ \\
\hline I doubt whether my practice should work with the MIS & -0.40 & 0.28 & -0.98 & -1.02 & $\mathrm{~N}>\mathrm{U}, \mathrm{I}$ \\
\hline
\end{tabular}

$\mathrm{U}=$ users, $\mathrm{I}=$ intenders, $\mathrm{N}=$ non-intenders

Differences and significance levels for each attitude item are shown in Table 3.

Intenders and users had a significantly more positive attitude toward using the MIS than 
non-intenders. Intenders and users were more convinced of the positive outcomes, relative advantage, and compatibility than non-intenders. Non-intenders regarded the MIS as more complex than intenders and users did. More so than non-intenders, intenders thought the visibility of the impact of the MIS was easy to assess. Users were more positive about the trialability of the MIS than non-intenders. Users and intenders were significantly less ambivalent than non-intenders.

Table 4 shows that both intenders and users perceived a significantly more positive norm from their social environment than non-intenders.

Table 4. Means of and significant differences in social influence items for non-intending, intending and using practices

\begin{tabular}{|c|c|c|c|c|c|}
\hline Variable (scale) & $\begin{array}{c}\text { Overall } \\
(N=251)\end{array}$ & $\begin{array}{c}\text { Non- } \\
\text { intenders } \\
(\mathrm{n}=118)\end{array}$ & $\begin{array}{l}\text { Intenders } \\
(n=42)\end{array}$ & $\begin{array}{l}\text { Users } \\
(n=91)\end{array}$ & $\begin{array}{l}\text { Tukey } \\
\text { HSD }\end{array}$ \\
\hline Perceived norm $(-2=$ negative norm,$+2=$ positive norm $)$ & 0.77 & 0.39 & 1.10 & 1.10 & $\mathbf{N}<\mathbf{U}, \mathbf{I}$ \\
\hline General practitioners & 0.57 & 0.21 & 0.87 & 0.89 & $\mathrm{~N}<\mathrm{U}, \mathrm{I}$ \\
\hline Gynecologists & 0.60 & 0.21 & 0.79 & 0.99 & $\mathrm{~N}<\mathrm{U}, \mathrm{I}$ \\
\hline Midwives & 0.83 & 0.32 & 1.31 & 1.25 & $\mathrm{~N}<\mathrm{U}, \mathrm{I}$ \\
\hline Pregnant women & 0.45 & 0.13 & 0.72 & 0.74 & $\mathrm{~N}<\mathrm{U}, \mathrm{I}$ \\
\hline Regional cooperation of midwives & 0.85 & 0.36 & 1.31 & 1.25 & $\mathrm{~N}<\mathrm{U}, \mathrm{I}$ \\
\hline Royal Dutch Organization for Midwives & 1.36 & 1.14 & 1.62 & 1.51 & $\mathrm{~N}<\mathrm{U}, \mathrm{I}$ \\
\hline Perceived support $(-2=$ discouragement,$+2=$ support $)$ & 0.57 & 0.44 & 0.71 & 0.67 & $\mathbf{N}<\mathbf{U}, \mathbf{I}$ \\
\hline General practitioners & 0.37 & 0.30 & 0.38 & 0.45 & \\
\hline Gynecologists & 0.39 & 0.31 & 0.44 & 0.46 & \\
\hline Midwives & 0.67 & 0.49 & 0.85 & 0.82 & $\mathrm{~N}<\mathrm{U}, \mathrm{I}$ \\
\hline Pregnant women & 0.35 & 0.21 & 0.56 & 0.45 & $\mathrm{~N}<\mathrm{U}, \mathrm{I}$ \\
\hline Regional cooperation of midwives & 0.71 & 0.55 & 0.90 & 0.81 & $\mathrm{~N}<\mathrm{U}, \mathrm{I}$ \\
\hline Royal Dutch Organization for Midwives & 0.91 & 0.80 & 1.07 & 0.97 & \\
\hline \multicolumn{6}{|l|}{ Modeling } \\
\hline $\begin{array}{l}\text { Perceived percentage of other midwives using the MIS } \\
(0-100 \%)\end{array}$ & 28 & 21 & 29 & 36 & $\begin{array}{l}\mathrm{N}<\mathrm{U}, \mathrm{I} \\
; \mathrm{I}<\mathrm{U}\end{array}$ \\
\hline Self-efficacy $(-3=$ definitely not,$+3=$ definitely $)$ & -0.50 & -0.69 & -0.36 & -0.33 & $\mathbf{N}<\mathbf{U}, \mathbf{I}$ \\
\hline \multicolumn{6}{|l|}{$\begin{array}{l}\text { Could/do you manage to use the MIS protocol to help } \\
\text { pregnant women quit smoking, when.....? }\end{array}$} \\
\hline - your consulting hours run over time & -0.88 & -1.19 & -0.93 & -0.47 & $\mathrm{~N}<\mathrm{U}, \mathrm{I}$ \\
\hline - you do not have enough time per client & -1.00 & -1.40 & -0.90 & -0.56 & $\mathrm{~N}<\mathrm{U}$ \\
\hline - your client is not interested & -1.46 & -1.58 & -1.14 & -1.47 & \\
\hline - your client resists talking about smoking & -1.46 & -1.56 & -1.12 & -1.51 & \\
\hline - your client has a low level of education & -0.05 & -0.36 & 0.40 & 0.13 & $\mathrm{~N}<\mathrm{U}, \mathrm{I}$ \\
\hline - your client has a high level of education & 0.70 & 0.48 & 0.88 & 0.89 & $\mathrm{~N}<\mathrm{U}, \mathrm{I}$ \\
\hline - your colleagues don't use the MIS protocol & -0.39 & -0.59 & -0.55 & -0.08 & $\mathrm{~N}<\mathrm{U}$ \\
\hline $\begin{array}{l}\text { - you experience discouragement from your colleagues } \\
\text { to work with the MIS }\end{array}$ & -0.93 & -1.03 & -1.29 & -0.66 & $\mathrm{I}<\mathrm{U}$ \\
\hline - your client has problems communicating in Dutch & -1.01 & -1.15 & -1.02 & -0.82 & \\
\hline - your client already has one or more children & 0.11 & -0.16 & 0.62 & 0.22 & $\mathrm{~N}<\mathrm{U}, \mathrm{I}$ \\
\hline - you take over a client from one of your colleagues & 0.30 & 0.08 & 0.52 & 0.46 & $\mathrm{~N}<\mathrm{U}, \mathrm{I}$ \\
\hline $\begin{array}{l}\text { - your client experiences a lot of difficulties in her } \\
\text { private life }\end{array}$ & -0.63 & -0.77 & -0.36 & -0.57 & \\
\hline - your client indicates not to have much time & -0.58 & -0.76 & -0.36 & -0.46 & \\
\hline - you are not specially trained to do so & -0.34 & -0.40 & -0.50 & -0.21 & \\
\hline - you didn't use MIS for your previous smoking clients & 0.19 & 0.05 & 0.29 & 0.33 & \\
\hline
\end{tabular}

$\mathrm{U}=$ users, $\mathrm{I}=$ intenders, $\mathrm{N}=$ non-intenders 
Intenders and users also perceived more support from their environment, especially from their colleagues and clients. Intenders and users both perceived a significantly larger proportion of midwives to use the MIS than non-intenders did. Users also expected a significantly larger proportion to use the MIS than intenders did.

Both users and intenders had a higher level of self-efficacy about using the MIS than non-intenders, especially regarding the less problematic situations (see Table 4).

\subsubsection{Regression analyses}

Predisposing and information factors that significantly increased the likelihood of adoption were the presence of one or more practice assistants $(\beta=-0.52, p<0.01)$, having a small proportion of clients of foreign ethnic origin $(\beta=-0.39, p<0.01)$, having received information from the Royal Dutch organization for Midwives $(\beta=0.42, p<0.01)$, and not having received information from the Dutch expert center on Tobacco Control $(\beta=-0.30, p<0.05)$. These factors explained $15 \%$ of the likelihood of adoption of the MIS $\left(R^{2}=0.15\right)$.

The second regression analysis showed that two awareness factors significantly increased the likelihood of adoption, namely having much knowledge about the MIS $(\beta=1.16, p<0.01)$ and attaching importance to smoking cessation guidance for pregnant women $(\beta=0.91, p<0.01)$. These factors increased the likelihood of the adoption of the MIS to $48 \%\left(R^{2}=0.48\right)$.

The third analysis showed the motivation factors that significantly increased the likelihood of adoption. These factors were a low level of ambivalence $(\beta=-0.84, p<0.01)$, perceiving few disadvantages $(\beta=-0.72, p<0.01)$, perceiving positive norms $(\beta=0.66$, $p<0.01)$, perceiving a high proportion of midwives to use the MIS $(\beta=0.61, p<0.05)$, and perceiving many advantages $(\beta=0.52, p<0.05)$. All factors together were associated with $69 \%$ of the variance in adoption of the MIS $\left(R^{2}=0.69\right)$. Except from the information factors all the other variables remained significant next to the motivational factors.

\subsection{Discussion}

The present study examined determinants of the adoption of a minimal-contact smoking cessation intervention by midwifery practices in the Netherlands. Users, intenders and non-intenders were compared in terms of psychosocial and organizational characteristics. A comprehensive set of theory-based determinants was assessed.

Hardly any differences between users, i.e., those who had already adopted and were using the intervention, and intenders, i.e., those who had adopted the intervention but were not using it yet, were found. Intenders and users only differed significantly on the concepts knowledge and modeling. Users had significantly more knowledge on the MIS and perceived a higher proportion of midwives to use the MIS. Both intenders and users differed significantly from non-intenders on all awareness and motivational 
concepts, except from risk perception.

To identify the factors with the strongest association with adoption, intenders and users were combined into one adoption group and compared with the non-intenders. Significant predisposing and information factors explained only 15 percent of the likelihood of adoption. This might mean that we did not assess all predisposing characteristics that distinguish between midwifery practices. It is also possible that the organizational characteristics that are most relevant to adoption are very similar in all midwifery practices. Personal awareness and motivation factors explained the likelihood of adoption much better than the predisposing organizational factors and information factors.

The most important predisposing factor that was found to facilitate adoption of the MIS was the availability of one or more assistants in the practice. One might think that the presence of assistants would result in lower workload and therefore facilitate adoption. However, correlations between the presence of assistants in a practice and amount of workload were very weak. This lack of association might be caused by the fact that assistants more often work in larger practices, where they especially perform organizing tasks that become more complex and time consuming when more midwives work in one practice (Kenens \& Hingstman, 2004). Despite the fact that workload does not show to be associated with the presence of assistants, the factor time might still be related to the association between having assistants and adoption. Practice assistants might be able to reduce the necessary time investment of midwives when carrying out the protocol (the MIS); they can play a role in organizing and executing the MIS. Furthermore, they can motivate midwives to use the MIS in the practice. Unfortunately, we have no further information on this issue.

The second predisposing factor that was significantly related to adoption was the proportion of clients of foreign ethnic origin in the practice. A large proportion appeared to impede adoption. This may be due to the low self-efficacy of midwives toward using the MIS when clients have problems communicating in Dutch. It may also be caused by perceived cultural differences that keep midwives from talking about smoking. Another reason why language problems and cultural differences might impede adoption is that these factors might create more work and less time to learn about and incorporate smoking prevention activities. Only a small relation $(r=0.15, p<0.05)$ between the proportion of clients of foreign origin and the proportion of clients with low educational levels was found.

A third predisposing factor, formalization, was not found to significantly increase the explained variance in adoption compared to a model with those two factors, but formalization increased among adoption stages and non-intenders and users differed significantly. This result partly contradicts with Rogers' theory, which states that 
formalization inhibits initiation in the innovation process (Rogers, 1995). However, our finding that more formalized practices not only adopted but also more often used the MIS than less formalized practices is in line with Rogers' view that formalization facilitates implementation of an innovation. One explanation may be that our study involved small organizations (mean size of 3.4 midwives), whereas Rogers refers to larger companies. This idea is supported by the findings of a comparable study on cardiac wards (mean size of 29 nurses), where formalization was indeed found to inhibit adoption of the MIS (Castle, 2001; Segaar, Bolman, Willemsen, \& De Vries, 2006). The relative small size of the organizations in our study might also have caused the lack of finding a positive association between size and adoption, which was found in several other studies (Castle, 2001; Moch \& Morse, 1977; Segaar et al., 2006; Sinclair, Maxfield, Marks, Thompson, \& Gershon, 2002).

The Royal Dutch Organization for Midwives was the most effective information channel to disseminate information about the MIS. It is likely that this source is highly trusted by midwives, since $90 \%$ of all midwives is member of this organization (Kenens \& Hingstman, 2004). Non-adopters were more likely than adopters to report that the Dutch expert center on Tobacco Control (STIVORO) provided them with information about the MIS. This difference can be attributed to the users. There were no differences between non-intenders and intenders. The lower likelihood of users to be informed by STIVORO may have been caused by the fact that STIVORO sent more direct mailing to non-users.

Two awareness factors were found to be significantly related to adoption, namely knowledge and personal relevance. This finding is in line with the theory that the innovation-decision process starts with the steps knowledge and persuasion and so can be regarded as preterm for adoption (Rogers, 1995).

Of the motivation factors attitude was found to be very strong associated with adoption. This is in line with the findings from several other studies (Bolman et al., 2002; McCarty, Hennrikus, Lando, \& Vessey, 2001; Mesters \& Meertens, 1999). The same is true for the positive association that we found between social influence and adoption (Paulussen et al., 1994; Wilhelm, 2002) and for the positive association between selfefficacy and adoption (Borrelli et al., 2001; McCarty et al., 2001; Park et al., 2003; Paulussen et al., 1994; Pullon et al., 2003; Vermette \& Godin, 1996). However, selfefficacy did not significantly increase the explained variance in adoption in a model with other motivational factors. This is probably due to its effect on attitude.

The best associative model of adoption stated the likelihood of adoption was highest when midwives had much knowledge about the MIS, they had a consistent positive attitude (perceiving a lot of advantages and few disadvantages, and low level of ambivalence), they perceived positive social norms toward adoption and perceived a high proportion of midwives to use the MIS, they had one or more practice assistants and 
there was a small proportion of clients of foreign ethnic origin. This model was associated with $69 \%$ of the variance in adoption.

Before we can conclude that the factors in the model are the factors which should be targeted when trying to improve adoption strategies for Minimal Intervention Strategies or other comparable interventions by midwives, we should overview the limitations of the present study.

First, the study yielded only cross-sectional data, precluding causal inferences. Awareness and motivation characteristics may have been influenced by use of the intervention. However, our research only found differences between users and intenders on the concepts knowledge and modeling. If this difference was caused by using the MIS, the present study might overrate the effect of knowledge and modeling on adoption. Since both intenders and users had more knowledge and perceived a higher proportion of midwives to use the MIS than non-intenders the existence of an important effect of knowledge and modeling on adoption is beyond dispute. No differences were found on any of the other variables. This suggests that most differences between adopters and non-adopters do not result from using the MIS, making it likely that there is a causal relationship between adoption and the determinants we found in this study.

Second, although we invited all midwifery practices in the Netherlands to participate, little more than half (57\%) enrolled, which may have resulted in selection bias. We compared the proportion of MIS users among our responders with the proportion of MIS users in the total population, which is registered in an electronic database by the organization that coordinates the implementation of the MIS (the Dutch expert center on Tobacco Control). At the time of our study, the registered proportion of users in this database was $35 \%$, while the proportion of users among our responders was $36 \%$. This indicates that practices using the MIS were not overrepresented. However, the proportion of smokers among the responders was $8 \%$, which is much smaller than the proportion of smokers among Dutch women in general (25\% in 2004 (TNS NIPO, 2005)). Even though midwives have been found to smoke less than the general female population (Sacker, 1990) the figure of $8 \%$ is small. This might indicate under representation of smokers among the respondents, which could have caused an underestimation of the effect of smoking on the adoption of the MIS.

In conclusion, the study has provided important insights into the factors that play a role in the adoption of a protocol for smoking cessation guidance in midwifery practices. These results could be used to improve dissemination strategies and facilitate adoption. However, there is still much room for improvement in this field, by further increase of knowledge on predictors of adoption. Therefore, studies like the present one should also be conducted in other settings. Ideally, these will be studies with a 
longitudinal design, planned prior to the implementation of interventions, so that predictive rather than associative patterns can truly be identified.

Practice implications of our findings are that, independent of what organizational characteristics occur, dissemination strategies should primarily focus on the awareness and motivation factors. Information should be disseminated to increase knowledge about the MIS. The positive attitude towards the MIS should also be stimulated by increasing the perception of advantages and by promoting the conviction that there are no disadvantages. The best way to disseminate information on the MIS and its advantages is via existing sources that are trusted by midwives, like professional associations.

Increasing the perception of advantages and decreasing that of disadvantages will also result in a desirable decrease in ambivalence. Furthermore, dissemination strategies should try to increase the positive perception of the social norms and the perceived number of other midwives using the MIS, for example by stimulating peer contacts on the subject. 



\section{CHAPTER 5}

Adherence to a Minimal-Contact Smoking

Cessation Intervention by midwives 


\section{Abstract}

Although smoking cessation programmes provided during pregnancy have proved to be efficacious in reducing smoking, health care professionals generally underuse these types of health promotion innovations. Adherent use of an innovation is a precondition for achieving the intended health impact. This chapter reports on the adherence to a protocol for smoking cessation guidance by midwives (MIS) and identifies psychosocial and organizational determinants of adherence to this protocol. A cross-sectional questionnaire survey was conducted among a random sample of 250 midwives who were trained in using the protocol, of whom 137 (55\%) responded. Questions on the determinants of adherence were based on the Integrated Change Model, which includes a wide range of factors, ranging from organizational to motivational and ability factors. Results show that almost all midwives asked their clients about their smoking behavior, advised them to quit smoking, assessed their motivation and discussed the reasons to quit. MIS elements that were less consistently applied included discussing barriers to smoking cessation, setting a quit date with motivated clients and providing follow-up care. Important differences between adherent and nonadherent users of the MIS were found. In conclusion, implementation strategies of future smoking cessation programmes should enhance midwives' efforts regarding follow-up care, discussing barriers to smoking cessation and setting a quit date with motivated clients, e.g. by improving their skills. Consistent use of intervention cards or schematic registration systems should be stimulated, and perceived support must increase.

\section{Submitted as:}

Segaar, D., Bolman, C., Willemsen, M.C., De Vries, H. Adherence to a Minimal-Contact Smoking Cessation Intervention by midwives. 


\subsection{I ntroduction}

Smoking during pregnancy is associated with serious health consequences, such as increased risk of preterm delivery and infant deaths (US Department of Health and Human Services, 2004; British Medical Association, 2004). For this reason, pregnant smokers are recommended to quit and smoking cessation guidelines for health professionals recommend providing pregnant smokers with short smoking cessation interventions based on the Ask, Advice, Assess, Assist and Arrange (5 A's) Model (Fiore et al., 2000; Raw, McNeill, \& West, 1999, Van Weel et al., 2005).

Although smoking cessation programmes provided during pregnancy have proved efficacious in reducing smoking rates among pregnant women (Lumley, Oliver, \& Waters, 2004), efficacious health promotion interventions like these are generally underused by health care professionals (Cranney, Warren, Barton, Gardner, \& Walley, 2001; Glasgow, Lichtenstein, \& Marcus, 2003; Haines \& Donald 1998) due to difficulties in implementing the interventions as intended (Hajek et al., 2001; Moore et al., 2002).

The study described here aimed to assess the implementation of a Dutch smoking cessation protocol for pregnant women, and to assess differences between those who do and do not adhere to the protocol.

\subsection{Background}

\subsubsection{Minimal Intervention Strategy for smoking cessation by midwives}

Approximately $18 \%$ of pregnant women in the Netherlands are smokers when they become pregnant (Lanting, Van Wouwe, \& Crone, 2005). In the Netherlands, midwives provide most pregnancy care. They assist about $75 \%$ of all pregnant women (TNO Preventie en Gezondheid, 2000). For most women, midwives are the main source of information on pregnancy-related subjects. From their third month of pregnancy, women visit their midwife regularly, at least once a month. Thus, midwives are regarded as ideal intermediaries to apply smoking cessation interventions for pregnant women. More than $90 \%$ of all Dutch midwives work in group practices of about three midwives. About half of them have practice assistants who take over some tasks, such as administrative tasks, answering the phone and preparing consultations (Kenens \& Hingstman, 2004). Between 1994 and 2000, a smoking cessation and relapse prevention protocol (the socalled Minimal Intervention Strategy or MIS) for midwives was developed. This protocol provides a stepwise strategy that focuses on the motivation of pregnant women to quit smoking and is comparable to the 5 A's Model. The protocol was found to be more effective than usual care in achieving smoking cessation six weeks post-intervention (7day abstinence $19 \%$ vs. $7 \%$ ) and six weeks post-partum (7-day abstinence $21 \%$ vs. 12\%) (De Vries, Bakker, Dolan-Mullen, Van Breukelen, in press). In 2002, the Dutch 
expert centre on tobacco control (STIVORO), together with the Dutch association of midwives (KNOV) started the nationwide distribution of the protocol.

\subsubsection{Diffusion}

Underuse of newly developed interventions is often the result of failures in the diffusion process. The diffusion process of innovations can be divided into three main phases: adoption, implementation and maintenance (Rogers, 1995). Adoption is the decision to make full use of an innovation as the best course of action; implementation occurs when an innovation is actually put into use; and maintenance is the phase in which continued use of the innovation is ensured. Various disciplines, ranging from marketing to psychology, have studied aspects of the diffusion of innovations. Such studies - which have tended to focus mostly on adoption, rather than on implementation or maintenance - have identified a wide variety of factors that may influence the diffusion of innovations, such as the size of the organization (Castle, 2001; Moch \& Morse, 1977), characteristics of the innovation itself (Rogers, 1995) and characteristics of the target group, like attitudes or beliefs (Bolman, De Vries, Van Breukelen, 2002a; Mesters \& Meertens, 1999; Michie, Hendy, Smith, \& Adshead, 2004). However, most of these studies were performed outside the field of public health, and often failed to provide information on generalizability to other settings (Foy, Eccles, \& Grimshaw, 2001; Glasgow, Lichtenstein, \& Marcus, 2003). Few studies have been conducted on the diffusion of smoking cessation interventions among midwives. Bakker and colleagues (2003) concluded that the implementation of a smoking cessation protocol in routine midwifery care in the Netherlands was feasible, but did not study determinants of successful implementation. Cooke and colleagues (1996) found the use of smoking cessation interventions to be predicted by self-reported ability to intervene smoking, hospital characteristics such as hospital policy and size, and work pressure. However, since Dutch midwives are not connected to hospitals, hospital characteristics are not relevant factors in their case.

Besides the scarcity of studies on the diffusion of smoking cessation interventions among midwives, there was one more reason to undertake the present study. Because there is no interdisciplinary theoretical framework for the study of diffusion in health care settings, no comprehensive overview is available of factors that relate to diffusion.

\subsubsection{Theoretical framework}

The study of diffusion of innovations can be regarded as the study of behavior change. A comprehensive theoretical model of behavior change that integrates both organizational and psychosocial factors is the Integrated Change Model (or I-Change Model) (De Vries, Mesters, Van de Steeg, \& Honing, 2005; De Vries, Mudde, Leijs et al., 2003; De Vries, Mesters, Van 't Riet, Willems, \& Reubsaet, 2006). The I-Change Model (see Figure 1) 
distinguishes three phases in the behavioral change process: a pre-motivational phase in which someone becomes aware of a particular problem and the need for new or altered behavior, a motivational phase in which the intention to engage in the behavior is formed and a post-motivational phase in which the intention is transformed into behavior. The application of this model in diffusion research is described more extensively elsewhere (Segaar, Bolman, Willemsen, \& De Vries, 2006).

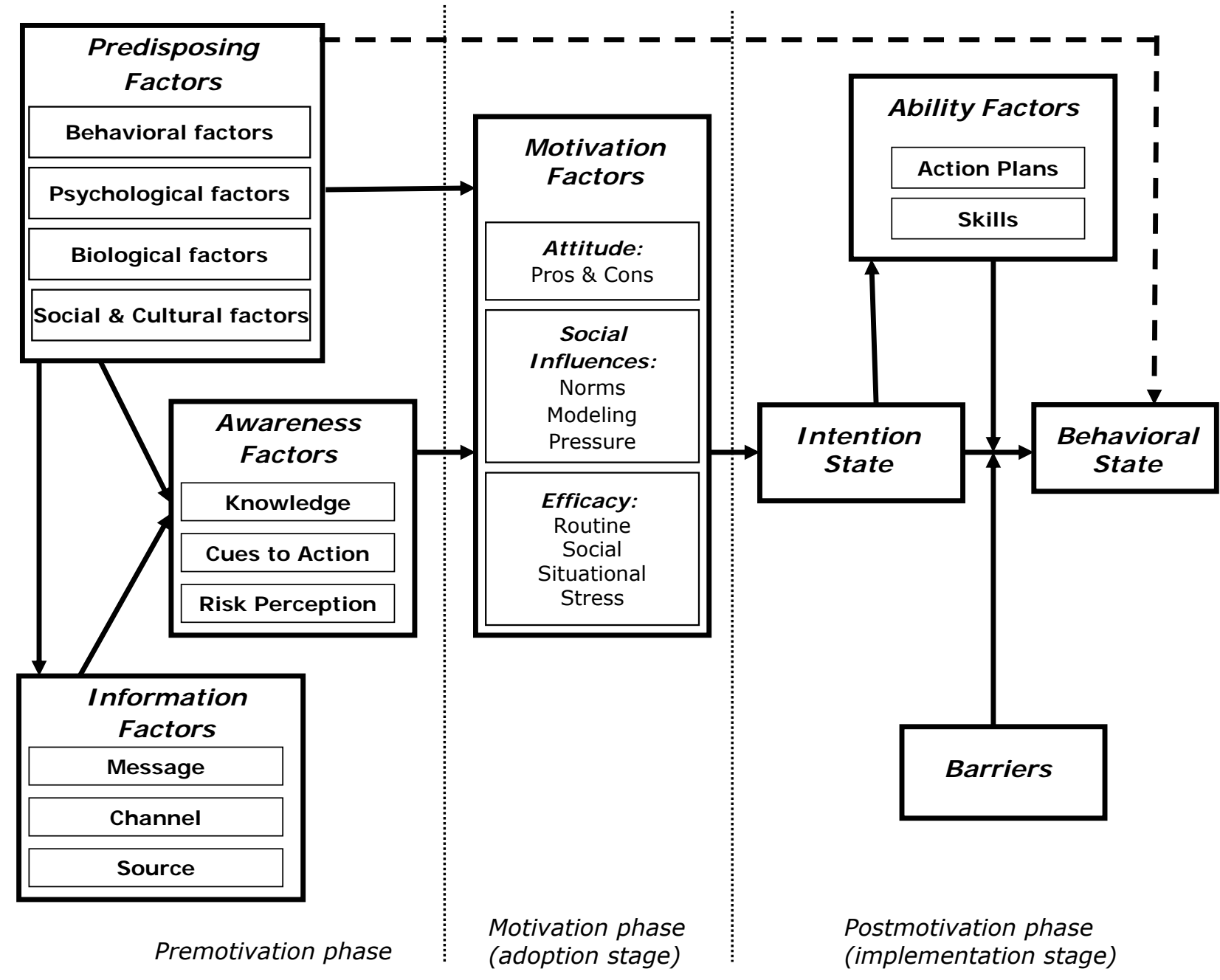

Figure 1. The I-Change Model to study implementation of the V-MIS.

\subsection{The study}

The effectiveness of a protocol can only be ensured if health care providers not only use but also accurately adhere to the protocol. The current paper describes a study relating to the second phase of the diffusion process, i.e. implementation of the MIS protocol by individual midwives, focusing on adherence to the protocol. A study of the factors that facilitate and impede the first of the three diffusion processes, i.e. adoption of the MIS 
among midwifery practices, using the same theoretical model, has been described elsewhere (Segaar, Bolman, Willemsen, De Vries, in press).

\subsubsection{Aims}

Our first main study aim was to assess adherence to the MIS protocol by midwives and to identify the steps of the protocol that were implemented least consistently. The second main study aim was to describe differences between adherent and nonadherent users of the MIS in terms of organizational and psychosocial factors. The I-Change model was used to define the factors that might be related to adherence to the MIS and therefore would differ between adherent and adherent users.

A secondary goal of the study was to assess the applicability of the model that we used to find factors related to adherence, i.e. the I-Change Model, and to assess the level of association between adherence and the factors used in the I-Change Model.

\subsubsection{Design}

A cross-sectional study was conducted among midwives in January 2005, three years after the start of the nationwide dissemination of the MIS. At that time, about $46 \%$ of Dutch midwives were using the MIS (Segaar \& Willemsen, 2005).

\subsubsection{Participants}

Study subjects were midwives from practices that had been trained in the use of the MIS and had ordered the necessary educational materials, prior to the study. A random sample of 250 midwives was approached for the study, selected from the 497 midwives who met the above criteria. SPSS was used for the randomization procedure.

\subsubsection{Data collection}

Data were collected from the participants by means of written questionnaires. In February 2005, written questionnaires were sent to the participants, accompanied by a cover letter and a postage-paid return envelope. The letter provided information about the purpose of the study and ensured confidentiality. We tried to prevent midwives from giving socially desirable answers by stating that we were especially interested in the difficulties experienced when using the MIS, in order to identify aspects that could be improved. Respondents could win a $€ 25$ voucher if they returned the questionnaire. Postal reminders were sent to non-responders three weeks after the first mailing and remaining non-responders were reminded by phone two weeks after the postal reminders. 


\subsubsection{Questionnaire}

The questionnaire was based on existing questionnaires or relevant scales, and on information from literature reviews and earlier work (Bakker, 2001; Bolman, De Vries, \& Mesters, 2002; Bolman, De Vries, \& Van Breukelen, 2002a; Mesters \& Meertens, 1999; Steckler, Goodman, McLeroy, Davis, \& Koch, 1992). The various measures are described below and in Tables 1 to 5 . Scale scores were calculated by averaging the scores of items that assessed the same variable, unless indicated otherwise. If scales were calculated, Cronbach's a (for three or more items) or the correlation coefficient $r$ (for two items) is presented.

\subsubsection{Outcome variable}

The outcome variable, i.e. adherence to the MIS, was assessed by 15 questions. In a previous phase of the study, the elements of the MIS that were relevant had been identified by a group of five experts in the fields of health psychology, health education, midwifery and smoking. Two of them had also been involved in the development of the MIS. For each of 15 important elements of the MIS, midwives were asked to indicate how often they performed them. Items were rated on a five-point scale $(0=$ never, $4=$ always). Subsequently, a total score for adherence was calculated by adding up the 15 item scores. In accordance with the views of an expert panel, which concluded that striving to use a guideline 'all of the time' may be too much to expect but 'most of the time' should be the goal (Gross et al., 2001), a cut-off point was defined at three quarters of the maximum value of 60 . If the adherence score was 45 or more, the midwives were categorized as 'adherent users', otherwise they were classified as 'nonadherent users'.

\section{Premotivational factors}

Detailed information on the questions about predisposing factors is provided in Table 1.

Biological factors were age and gender of the midwife. Behavioral factors were smoking behavior, assessed according to Dutch guidelines (Mudde, Willemsen, Kremers, \& De Vries, 2000), the number of years they had been employed as a midwife, the number of working hours per week and their personal level of innovativeness. Social and cultural factors were assessed by questions related to the environmental and organizational context and the style of decision-making (Koopman \& Pool, 1991; 1994). Environmental context included the following characteristics of the pregnant women; ethnic background, educational level and smoking status at the first visit. Organizational context characteristics included the number of midwives in the practice, the number of pregnancies monitored per year, the presence of practice assistants, the time available for the first visit, and the perceived workload. Style of decision making was assessed by 
five items: centralization (decisions entirely made by the individual midwife or centralized among cooperating midwives); formalization of decision-making (own solutions or set procedures); the amount of information gathered before decisions are made; the proportion of external information sources versus internal information sources consulted for decisions, and the way disagreements are solved. An additional question was asked about the level of disagreement that occurred in the working environment when a decision concerning the MIS had to be made. Furthermore, Table 1 shows detailed information on the questions about information factors. The midwives were asked if they consulted each of four information sources.

Detailed information on the questions about awareness is provided by Table 2 . Knowledge about the MIS was assessed by two questions $(r=0.77)$. Cues to action were assessed by two subscales: personal relevance ( 5 items, Cronbach's $a=0.60$ ) and dissatisfaction with the current method of smoking cessation guidance ( 2 items, $r=0.61$ ). Risk perception was assessed by four questions about the perceived risks of smoking to the babies and the pregnant women (Cronbach's $a=0.71$ ).

\section{Motivational factors}

Detailed information on the questions about motivational factors is provided in Tables 3 and 4.

Attitudes (Table 3) were assessed by 24 questions, including 9 items on beliefs about the innovation characteristics (Rogers 2002, Steckler et al., 1992) and 15 items on perceived health benefits and other outcome expectations (Bandura, 1986) of using the MIS protocol (Bakker et al., 2003). A factor analysis with oblimin rotation on the attitude variables yielded two attitude factors, viz. the pros (12 items, Cronbach's $\mathrm{a}=0.85$ ) and cons (12 items, Cronbach's $\mathrm{a}=0.84$ ) of using the MIS protocol. Attitude ambivalence (Ajzen, 2001) was assessed by two questions ( $r=0.78)$.

Perceived social influences (Table 4) were assessed by means of three constructs: perceived social norms, perceived social support and social modeling (De Vries et al., 1994). Perceived norms and support were assessed for seven subgroups of variables (see Table 4). Since both constructs were designed to assess the perceived opinions and behavior of several persons together and the correlation between the items may therefore be low, indexes were constructed instead of scales, by summing the scores of the subgroups. Social modeling was assessed by one question.

Self-efficacy expectations (Table 4) were assessed by asking midwives whether they would succeed in providing smoking cessation guidance with the MIS in 12 situations occurring in daily practice. Situations were derived from previous research on the diffusion of smoking cessation programmes (Bakker et al., 2003, Cooke et al., 2000). Factor analysis yielded four self-efficacy factors, viz. stress self-efficacy ( 2 items, 
$r=0.83$ ); situational self-efficacy - operationalized as (1) efficacy in the case of resistant patients ( 2 items, $r=0.80$ ) and ( 2 ) efficacy with regard to specific patient characteristics - (4 items, Cronbach's $a=0.86$ ) and social self-efficacy ( 2 items, $r=0.78$ ). Two items did not fit any of the factors and were therefore not included in any scale.

\section{Postmotivational factors}

Detailed information on the questions about ability factors and barriers is provided in Table 5. Midwives were asked if they thought they had sufficient skills to apply eight aspects of the MIS (Cronbach's $\mathrm{a}=0.82$ ). As actions plans are difficult to measure retrospectively, we measured the related concept goal actions, which can be defined as "sub-actions" that faciltate the achievement of the intended final goal (behavior). Goal actions were assessed by asking how often midwives performed each of three actions that facilitate use of the MIS (e.g. use of the MIS intervention card when applying the MIS or consulting a colleague in case of problems with applying the MIS). Factor analysis and correlation analysis revealed that only the two goal actions that were related to consulting colleagues correlated quite high. So these two were averaged to form one scale $(r=0.60)$. The other two goal actions were separately included in the analyses. The only barrier we assessed was the availability of four types of material that had to be available to be able to work with the MIS. An index was calculated by adding up the four scores.

\subsubsection{Data analysis}

Descriptive analyses were used for our study's first main aim, i.e. to assess adherence to the MIS protocol and to assess which steps of the protocol were the most difficult to implement. With respect to the second main aim of the study, t-tests were used to assess differences between adherent users and nonadherent users of the MIS for all factors of our research model (Figure 1). Chi-square tests were used for dichotomous variables. Pearson correlations with adherence were computed for all variables.

With respect to our secondary goal, i.e. to assess the applicability of the I-Change model in research on protocol use by midwives, a sequential logistic regression analysis was performed, with adherence as the outcome. First, the premotivational factors, i.e. predisposing, information and awareness factors were entered into the model. Motivational factors were then added to the model, using the enter procedure, and finally the postmotivational factors, i.e. ability factors and barriers, were entered. The results of these analyses indicated to what extent the factors in the Integrated Change Model were associated with adherence to the MIS protocol. The sequential procedure was used to find out whether motivational factors added significant explanatory value to premotivational factors and whether they mediated the association between 
premotivational factors and behavior, as is assumed in the I-Change model. This analysis also examined whether postmotivational factors added significant explanatory value to premotivational and motivational factors in predicting adherence. Because of the large number of variables relative to the number of respondents, we were not able to include all variables in the logistic regression analysis, so our analysis included only variables with $\mathrm{p}<0.20$ in the t-test or Chi-square test (Tabachnick \& Fidell, 2001). Standardized Z-scores of the variables were used to compare the magnitude of the partial association between variables, based on their standardized beta values ( $(B)$. Nagelkerke's $\mathrm{R}^{2}$ was used to approximate the levels of association of factors with adherence. Assumptions for regression analyses and a check on multicollinearity between the independent variables proved satisfactory. Differences were considered significant when $p<0.05$. All analyses were performed using SPSS version 13.0.

\subsection{Results}

\subsubsection{Sample description}

A total of 137 of the 250 midwives (55\%) returned the questionnaire. Thirty-three midwives indicated that they had never started using the MIS. They were excluded from the analyses of differences between adherent and nonadherent users. Of the remaining 104 midwives, one was excluded because she had not answered the questions about the outcome variable 'adherence to the MIS' in the questionnaire. The results presented below are therefore based on 103 questionnaires. Thirty-seven midwives (36\%) turned out to be nonadherent users of the MIS and 66 (64\%) were adherent users of the MIS.

\subsubsection{Adherence}

All 103 included midwives always asked their clients at the first consultation if they smoked, and if so how much they smoked on average. Sixty-five percent asked most $(\geq 75 \%)$ of their smoking clients about the smoking behavior of their partners. During the first consultation, almost all midwives advised most smokers to quit smoking (99\%), assessed their motivation to quit (97\%), and discussed the reasons to quit smoking (90\%). Furthermore, about $81 \%$ of the midwives provided most smokers with the 'Babyfit' information magazine (self-help material, part of the MIS intervention) but only $49 \%$ referred to this information magazine during later consultations. From this step onwards, adherence to the MIS protocol decreased. Whereas $72 \%$ discussed the difficulties of smoking cessation with most smokers, only $58 \%$ of the midwives usually set a quit date with clients who were motivated to quit and $53 \%$ usually discussed the option of using smoking cessation aids.

After the first consultation, $87 \%$ of the midwives repeatedly asked most of their motivated clients and $68 \%$ repeatedly asked most of their unmotivated clients if they 
smoked. Additionally, $69 \%$ of the midwives talked to most of their clients who quit smoking during pregnancy about preventing relapse after delivery, while $49 \%$ provided most of these clients with the information booklet about relapse prevention (part of the MIS intervention) following delivery.

Table 1. Comparison of midwives who adhere to the protocol with nonadherent midwives, with respect to predisposing and information factors ${ }^{1}$

\begin{tabular}{|c|c|c|c|c|}
\hline Variable (scale) & $\begin{array}{l}\text { Overall } \\
(N=10 \vdots\end{array}$ & $\begin{array}{c}\text { Non- } \\
\text { adherent } \\
\text { users }(n=37)\end{array}$ & $\begin{array}{l}\text { Adherent } \\
\text { users } \\
(n=66)\end{array}$ & p-value $\$$ \\
\hline \multicolumn{5}{|l|}{ Biological factors } \\
\hline Female (\% yes) & 100 & 100 & 100 & * \\
\hline Age (years) & 36 & 34 & 37 & \\
\hline Level of innovativeness (change: $-2=$ negative,$+2=$ positive) & 1.10 & 0.89 & 1.22 & $\mathrm{p}<0.05$ \\
\hline \multicolumn{5}{|l|}{ Behavioral factors } \\
\hline Smokes (\% yes) & 14 & 14 & 14 & $@$ \\
\hline Years of working as a midwife & 11 & 9 & 11 & \\
\hline Working hours per week & 39 & 36 & 40 & \\
\hline \multicolumn{5}{|l|}{ Social and cultural factors } \\
\hline \multicolumn{5}{|l|}{ Environment } \\
\hline Estimated percentage of clients of non-Dutch origin & 16 & 16 & 16 & * \\
\hline Estimated percentage of clients with a low educational level & 37 & 39 & 36 & * \\
\hline $\begin{array}{l}\text { Estimated percentage of clients who smoke at the time } \\
\text { of their first visit }\end{array}$ & 13 & 13 & 13 & * \\
\hline \multicolumn{5}{|l|}{ Organization } \\
\hline Number of midwives in the practice & 4.3 & 4.4 & 4.2 & \\
\hline Percentage of practices with assistants (\% yes) & 72 & 68 & 74 & * \\
\hline Number of pregnancies monitored per year & 302 & 285 & 306 & \\
\hline Time available for the first visit, in minutes & 37 & 34 & 39 & \\
\hline Workload $(-3=$ too low,$+3=$ too high $)$ & 0.52 & 0.46 & 0.55 & \\
\hline \multicolumn{5}{|l|}{ Decision-making styles $(0=l o w, 4=h i g h)$} \\
\hline Centralization level of decision & 2.76 & 2.59 & 2.83 & \\
\hline Formalization level & 2.28 & 2.05 & 2.40 & $\mathrm{p}<0.05$ \\
\hline Level of disagreement & 0.25 & 0.32 & 0.22 & \\
\hline $\begin{array}{l}\text { If disagreement occurs, it's solved through compromise } \\
\text { (\% yes) }\end{array}$ & 50 & 41 & 58 & * \\
\hline Amount of information used & 2.74 & 2.59 & 2.81 & \\
\hline $\begin{array}{l}\text { Type of information sources used ( } 0=\text { only internal, } \\
4=\text { only external sources) }\end{array}$ & 2.13 & 2.14 & 2.13 & \\
\hline Information factors (index $0-4$ ) & 2.64 & 2.55 & 2.68 & \\
\hline Skills training (\% yes) & 95 & 100 & 92 & * \\
\hline Colleague (\% yes) & 34 & 33 & 35 & * \\
\hline Instruction manual (\% yes) & 99 & 100 & 98 & * \\
\hline $\begin{array}{l}\text { Newsletter about MIS and smoking in presence of small } \\
\text { children (\% yes) }\end{array}$ & 36 & 21 & 42 & $\mathrm{p}<0.05^{*}$ \\
\hline
\end{tabular}

${ }^{1}$ The value in table is the mean value, unless indicated otherwise $\$ p$-values for differences between means were tested with t-tests unless indicated otherwise, and are only presented if significant $*$ Differences between the groups were tested with chi-square tests ${ }^{\circledR}$ Fisher's exact test instead of Chi-square test was used, because of the small number of cases in one group.

\section{Premotivational factors}

Table 1 shows that there were only two significant differences between adherent and nonadherent users as regards predisposing factors. Adherent users were more 
innovative and were more likely to work in organizations with higher levels of formalization than nonadherent users. Furthermore, Table 1 shows that adherent and nonadherent users did not differ in the total number of information sources they used. The only significant difference was that adherent users were more likely to read the newsletter about the MIS than nonadherent users.

With respect to awareness factors Table 2 reveals that, compared to nonadherent users, adherent users had significantly more knowledge about the MIS, were more convinced of the importance of assisting pregnant women with smoking cessation, and were more likely to agree that smoking cessation assistance is a task of midwives (personal relevance). Adherent and nonadherent users were equally dissatisfied with their smoking cessation support before they started to use the MIS and did not differ significantly in their perceptions of the risks of smoking.

Table 2. Comparison of midwives who adhere to the protocol with nonadherent midwives, with respect to awareness factors ${ }^{1}$

\begin{tabular}{|c|c|c|c|c|}
\hline Variable (scale) & $\begin{array}{l}\text { Overall } \\
(\mathrm{N}=103)\end{array}$ & $\begin{array}{c}\text { Nonadherent } \\
\text { users } \\
(n=37) \\
\end{array}$ & $\begin{array}{c}\text { Adherent } \\
\text { users } \\
(n=66)\end{array}$ & $\begin{array}{c}\mathrm{p}- \\
\text { value }\end{array}$ \\
\hline Knowledge level ( $0=$ not at all, $4=$ very well) & 3.17 & 2.91 & 3.31 & $p<0.05$ \\
\hline Do you know what the MIS is? & 3.35 & 3.14 & 3.45 & $\mathrm{p}<0.05$ \\
\hline Are you familiar with all steps of the MIS? & 2.99 & 2.68 & 3.15 & $\mathrm{p}<0.05$ \\
\hline Personal relevance $(-2=$ disagree,$+2=$ agree $)$ & 1.30 & 1.11 & 1.40 & $\mathbf{p}<0.01$ \\
\hline Assisting pregnant women with smoking cessation is important & 1.55 & 1.35 & 1.67 & $\mathrm{p}<0.05$ \\
\hline $\begin{array}{l}\text { It is important that midwives assist pregnant women with } \\
\text { smoking cessation by midwives }\end{array}$ & 1.01 & 0.71 & 1.17 & $\mathrm{p}<0.01$ \\
\hline $\begin{array}{l}\text { Midwives play an important role in the decision of pregnant } \\
\text { women to quit smoking }\end{array}$ & 0.73 & 0.59 & 0.81 & \\
\hline It is important that pregnant women quit smoking & 1.93 & 1.94 & 1.92 & \\
\hline $\begin{array}{l}\text { Helping pregnant women to stop smoking is a task for } \\
\text { midwives }\end{array}$ & 1.26 & 0.97 & 1.41 & $p<0.01$ \\
\hline Dissatisfaction $(-2=$ disagree,$+2=$ agree $)$ & 0.49 & 0.40 & 0.54 & \\
\hline $\begin{array}{l}\text { Before the MIS was introduced, I thought my smoking } \\
\text { cessation support should be improved }\end{array}$ & 0.66 & 0.56 & 0.71 & \\
\hline $\begin{array}{l}\text { Before the MIS was introduced, I was not satisfied with the } \\
\text { support on smoking cessation I gave to women }\end{array}$ & 0.33 & 0.24 & 0.37 & \\
\hline Risk perception ( $1=$ no risk, $10=$ very high risk) & 8.63 & 8.54 & 8.71 & \\
\hline Perceived risk of smoking for the unborn & 9.02 & 8.81 & 9.15 & \\
\hline Perceived risk of smoking for a pregnant women's own health & 8.53 & 8.35 & 8.67 & \\
\hline $\begin{array}{l}\text { Perceived harmfulness to the baby of smoking during the } \\
\text { breastfeeding period }\end{array}$ & 8.05 & 8.03 & 8.09 & \\
\hline $\begin{array}{l}\text { Perceived harmfulness to the baby of smoking in the presence } \\
\text { of the baby }\end{array}$ & 8.91 & 8.97 & 8.92 & \\
\hline
\end{tabular}

\footnotetext{
${ }^{1}$ The value in table is the mean value ${ }^{\#} p$-values for differences between means were tested with t-tests unless
} indicated otherwise, and are only presented if significant

\section{Motivational factors}

Table 3 shows the scores of adherent and nonadherent users of the MIS on attitude items. The midwives generally perceived more pros than cons of the MIS, but adherent users had a more positive attitude toward the MIS than nonadherent users. 
Furthermore, they perceived less cons of using the MIS, for example in terms of time expenditure or applicability. Adherent users were also significantly less ambivalent, which means that they were more convinced of their opinion about the MIS.

Table 3. Comparison of midwives who adhere to the protocol with nonadherent midwives, with respect to attitude items ${ }^{1}$

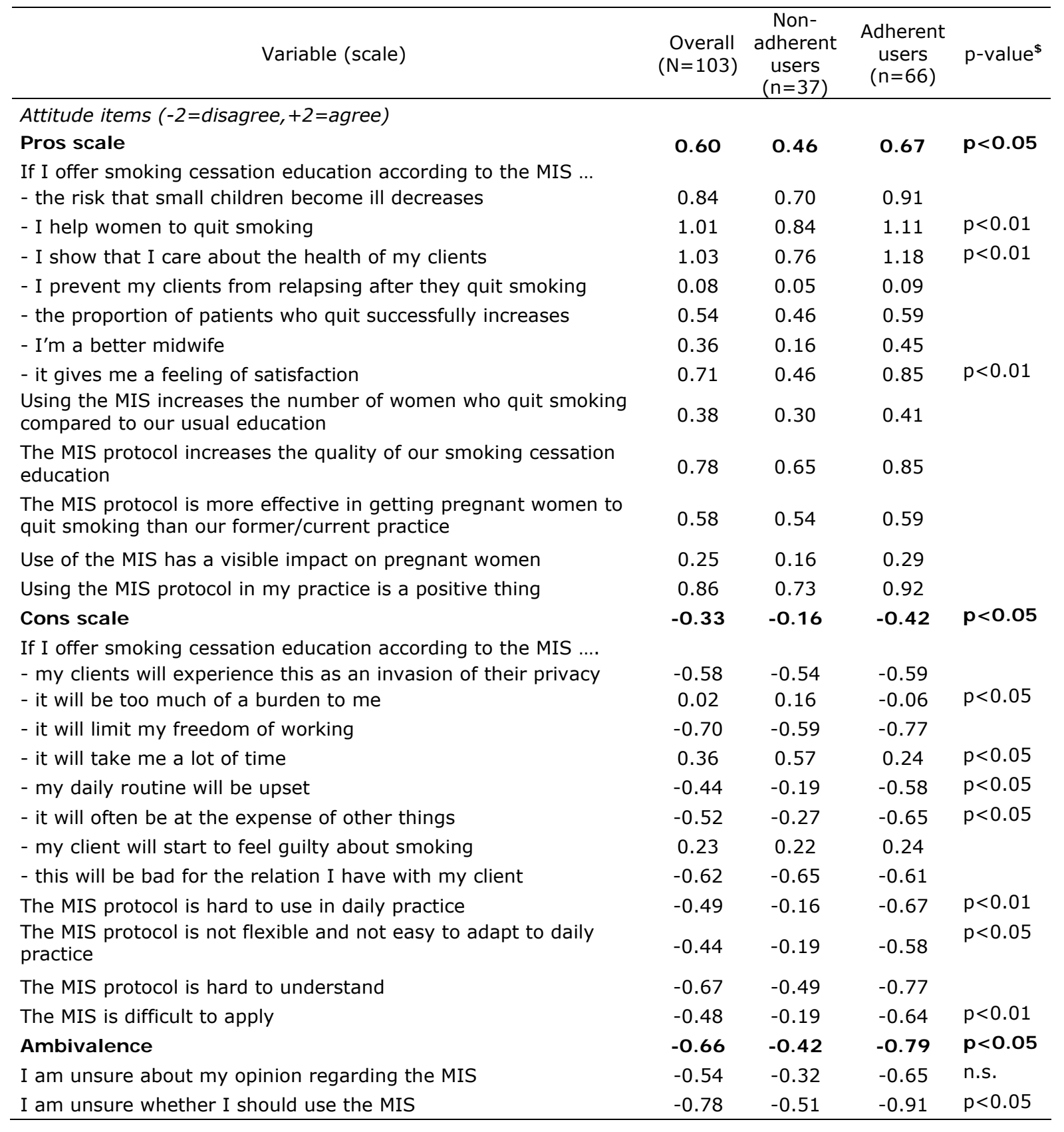

${ }^{1}$ The value in table is the mean value $\$ p$-values for differences between means were tested with $t$-tests unless indicated otherwise, and are only presented if significant

Table 4 shows the results for social influence and self-efficacy. It reveals that adherent users perceived more support from their social environment than nonadherent users, especially from other midwives, pregnant women and the regional association of 
midwives. With respect to perceived norms, it was only from other midwives that adherent users perceived more positive norms towards using the MIS than nonadherent users.

Table 4. Comparison of midwives who adhere to the protocol with nonadherent midwives, with respect to social influence and self-efficacy items ${ }^{1}$

\begin{tabular}{|c|c|c|c|c|}
\hline Variable (scale) & $\begin{array}{r}\text { Overall } \\
(\mathrm{N}=103)\end{array}$ & $\begin{array}{c}\text { Non- } \\
\text { adherent } \\
\text { users } \\
(n=37) \\
\end{array}$ & $\begin{array}{c}\text { Adherent } \\
\text { users } \\
(n=66)\end{array}$ & $p$-value $\$$ \\
\hline \multicolumn{5}{|l|}{ Social influence items } \\
\hline Social norm $(-2=$ negative norm, $2=$ positive norm, scale $=-14,14)$ & 6.33 & 5.23 & 6.88 & \\
\hline General practitioners & 0.82 & 0.59 & 0.94 & \\
\hline Gynaecologists & 0.79 & 0.57 & 0.89 & \\
\hline Midwives & 1.17 & 0.89 & 1.32 & $\mathrm{p}<0.01$ \\
\hline Pregnant women & 0.63 & 0.54 & 0.67 & \\
\hline Partners of pregnant women & 0.38 & 0.35 & 0.39 & \\
\hline Regional association of midwives & 1.14 & 0.97 & 1.23 & \\
\hline Royal Dutch Organization for Midwives & 1.40 & 1.32 & 1.44 & \\
\hline Social support $(-2=$ discouragement, $2=$ support, scale $=-14,14)$ & 2.85 & 1.68 & 3.44 & $p<0.01$ \\
\hline General practitioners & 0.20 & 0.11 & 0.24 & \\
\hline Gynaecologists & 0.23 & 0.11 & 0.29 & $\mathrm{p}<0.05$ \\
\hline Midwives & 0.65 & 0.43 & 0.77 & $p<0.05$ \\
\hline Pregnant women & 0.28 & 0.14 & 0.35 & \\
\hline Partners of pregnant women & 0.27 & 0.16 & 0.32 & \\
\hline Regional association of midwives & 0.61 & 0.27 & 0.79 & $\mathrm{p}<0.01$ \\
\hline Royal Dutch Organization for Midwives & 0.61 & 0.46 & 0.68 & \\
\hline \multicolumn{5}{|l|}{ Modelling } \\
\hline Perceived $\%$ of Dutch midwives who use the MIS $(0-100 \%)$ & 49 & 47 & 50 & \\
\hline \multicolumn{5}{|l|}{ Self-efficacy $(-3=$ definitely not,$+3=$ definitely $)$} \\
\hline \multicolumn{5}{|l|}{$\begin{array}{l}\text { Could/do you manage to use the MIS protocol to help } \\
\text { pregnant women quit smoking, when.....? }\end{array}$} \\
\hline Stress & -0.36 & -0.84 & -0.12 & $\mathbf{p}<0.05$ \\
\hline - your consulting hours run over time & -0.28 & -0.81 & -0.02 & $p<0.05$ \\
\hline - you do not have enough time per client & -0.43 & -0.86 & -0.23 & $p<0.05$ \\
\hline Situation: Resistant patient & -1.37 & -1.47 & -1.33 & \\
\hline - your client is not interested & -1.36 & -1.51 & -1.30 & \\
\hline - your client resists talking about smoking & -1.38 & -1.43 & -1.35 & \\
\hline Situation: Patient characteristics & 0.19 & -0.01 & 0.29 & \\
\hline - your client has a low level of education & 0.31 & 0.24 & 0.32 & \\
\hline - your client already has one or more children & 0.27 & 0.14 & 0.32 & \\
\hline - your client is experiencing a lot of difficulties in her private life & -0.30 & -0.57 & -0.14 & \\
\hline - you take over a client from one of your colleagues & 0.49 & 0.14 & 0.67 & $p<0.05$ \\
\hline Social & -0.55 & -0.51 & -0.55 & \\
\hline - your colleagues do not use the MIS protocol & -0.28 & -0.19 & -0.30 & \\
\hline - you experience discouragement from colleagues to use the MIS & -0.82 & -0.84 & -0.79 & \\
\hline \multicolumn{5}{|l|}{ Other } \\
\hline - your client has problems communicating in Dutch & -0.95 & -0.97 & -0.98 & \\
\hline - you did not use the MIS for your previous smoking clients & 0.53 & 0.30 & 0.68 & \\
\hline
\end{tabular}

${ }^{1}$ The value in table is the mean value $\$ p$-values for differences between means were tested with t-tests unless indicated otherwise, and are only presented if significant 
With respect to modeling, the perceived percentage of Dutch midwives that use the MIS did not differ significantly between the two groups. Only for stressful situations and when taking over a client from one of their colleagues did adherent users have higher self-efficacy than nonadherent users.

\section{Postmotivational factors}

With respect to ability factors Table 5 shows that adherent users were significantly more convinced that they had mastered the skills required to implement the different steps of the MIS than nonadherent users.

Table 5. Comparison of midwives who adhere to the protocol with nonadherent midwives, with respect to ability factors and barriers ${ }^{1}$

\begin{tabular}{|c|c|c|c|c|}
\hline Variable (scale) & $\begin{array}{c}\text { Overall } \\
(\mathrm{N}=103)\end{array}$ & $\begin{array}{c}\text { Non- } \\
\text { adherent } \\
\text { users } \\
(n=37)\end{array}$ & $\begin{array}{c}\text { Adherent } \\
\text { users } \\
(n=66)\end{array}$ & $\begin{array}{c}\mathrm{p}- \\
\text { value }\end{array}$ \\
\hline Skills $(-2=$ disagree,$+2=$ agree $)$ & 3.76 & 2.91 & 4.21 & $\mathbf{p}<0.01$ \\
\hline \multicolumn{5}{|l|}{ I have sufficient skills to... } \\
\hline -help pregnant women to stop smoking by means of the MIS & 0.47 & 0.14 & 0.64 & $\mathrm{p}<0.01$ \\
\hline -ask women about her smoking behavior & 1.43 & 1.39 & 1.45 & \\
\hline -advise women to quit smoking & 1.39 & 1.22 & 1.48 & $p<0.05$ \\
\hline -talk about the motivation to quit & 1.22 & 1.11 & 1.29 & \\
\hline -talk about the barriers to smoking cessation & 0.89 & 0.53 & 1.09 & $\mathrm{p}<0.01$ \\
\hline -address the use of smoking cessation aids & 0.39 & 0.14 & 0.52 & \\
\hline -set a date for quitting & 0.80 & 0.61 & 0.89 & \\
\hline -address relapse prevention after delivery & 0.92 & 0.66 & 1.06 & $\mathrm{p}<0.01$ \\
\hline \multicolumn{5}{|l|}{ Goal actions ( $0=$ never, $4=$ very often $)$} \\
\hline Use of intervention card ( $0=$ never, $4=$ always) & 1.92 & 1.32 & 2.26 & $\mathrm{p}<0.01$ \\
\hline Before seeing a patient, I think about how to apply the MIS & 0.82 & 0.59 & 0.95 & \\
\hline Goal actions related to colleagues & 0.96 & 0.68 & 1.12 & \\
\hline $\begin{array}{l}\text { I talk about the MIS with colleagues, when I think it can help } \\
\text { me to implement the MIS better }\end{array}$ & 0.80 & 0.62 & 0.90 & \\
\hline $\begin{array}{l}\text { If I have problems applying the MIS to one of my clients, I } \\
\text { consult one of my colleagues }\end{array}$ & 1.12 & 0.74 & 1.34 & $\mathrm{p}<0.05$ \\
\hline Availability of materials (index $0-4)^{\dagger}$ & 3.21 & 2.97 & 3.33 & \\
\hline Information magazine for pregnant women (\% yes) & 89 & 86 & 91 & \\
\hline Intervention card (\%yes) & 74 & 70 & 75 & \\
\hline Relapse prevention brochure (\% yes) & 75 & 65 & 79 & \\
\hline Videotape with information for pregnant women (\% yes) & 83 & 78 & 87 & \\
\hline
\end{tabular}

${ }^{1}$ The value in table is the mean value, unless indicated otherwise ${ }^{+}$sum of scores (yes $=1$ ) on availability of materials $\$ p$-values for differences between means were tested with t-tests unless indicated otherwise, and are only presented if significant

Adherent users were more likely to perceive themselves as able to help pregnant women with smoking cessation according to the MIS, to advise women to quit smoking, and to talk about barriers to smoking cessation. Furthermore, adherent users significantly more often performed two goal actions, namely they were more likely to consult one of their 
colleagues if they had problems applying the MIS to a particular client and they much more consistently used the intervention card than nonadherent users. With respect to barriers Table 5 shows that adherent users and nonadherent users did not differ in their reports on the availability of the necessary MIS materials.

\subsubsection{Levels of association}

Figure 2 presents the significant Pearson correlation coefficients between the potential determinants of adherence and adherence.

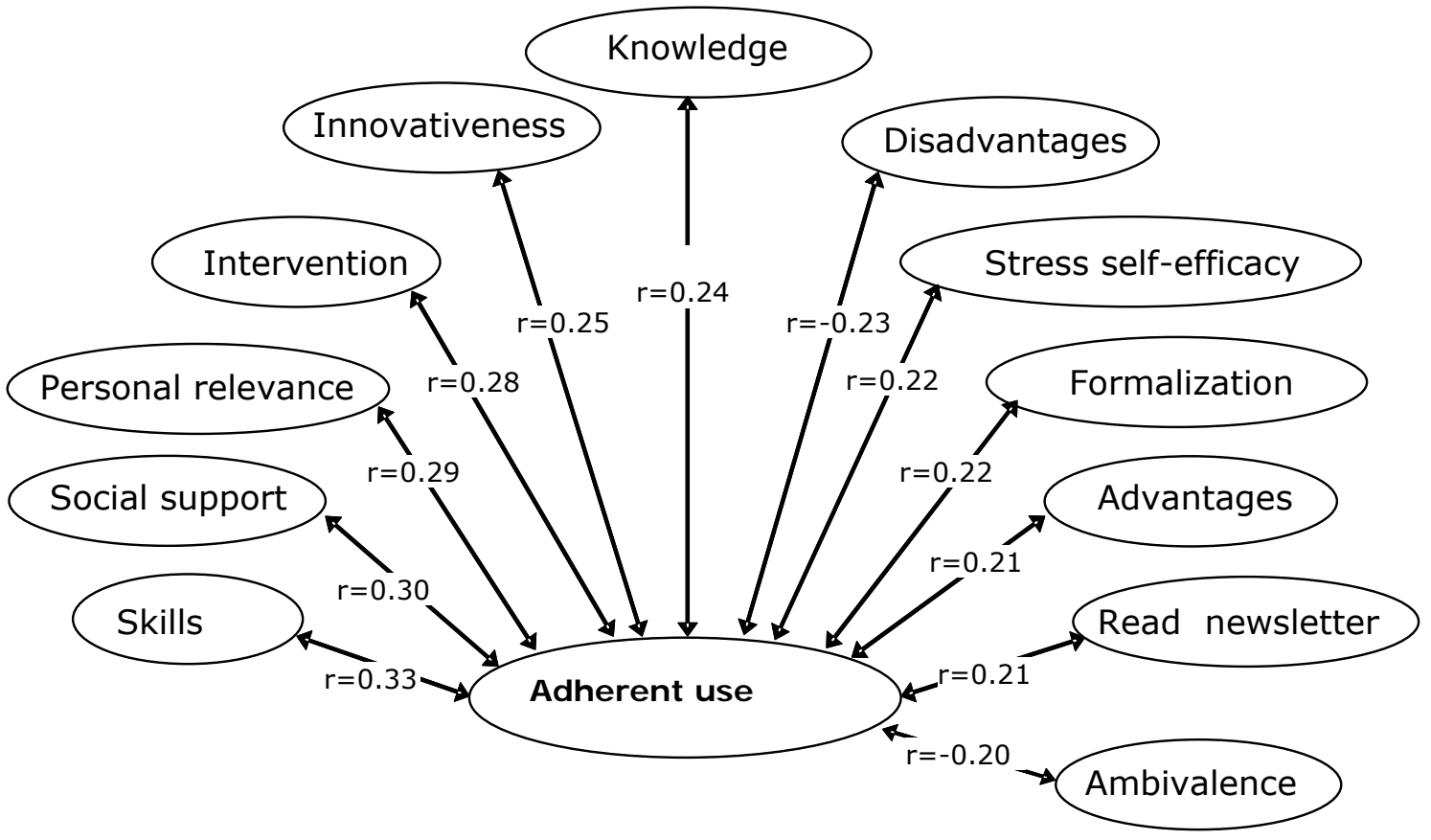

Figure 2. Significant pearson correlations with adherence

It shows from the left to the right side that adherence correlated most strongly with high perceived skills, followed by positive social support, high personal relevance, consequent intervention card use, high innovativeness, high knowledge level, perceiving fewer disadvantages, higher self-efficacy in stressful situations, higher organization formalization, perceiving more advantages, reading the MIS newsletter, and lower levels of ambivalence.

\subsubsection{Applicability of the I-Change Model}

The factors in the I-Change Model were associated with $56 \%$ of the variance in adherence to the C-MIS protocol among midwives. Premotivational factors were associated with $36 \%$ of the variance in adherence. Age $(B=0.68, p<0.05)$, innovativeness $(B=0.61, p<0.05)$, personal relevance $(B=0.60, p<0.05)$ and level of 
formalization ( $B=0.50, p<0.05)$ were significantly positively associated with adherence when the other premotivational factors were controlled for. Adding motivational factors increased the association with adherence to $46 \%$, though this increase was not significant $\left(\chi^{2}=10.4, p<0.25\right)$.

Only a small part of the association between premotivational factors and adherence was mediated by motivational factors. Only the association between personal relevance and adherence seemed to be highly mediated by motivational factors, since its B dropped substantially after motivational factors were added to the model. The other premotivational factors showed a significant partial association with adherence when motivational factors were also included in the model. Perceived support was the only motivational variable with a significant partial association with adherence when the other factors were also included in the model $(B=0.76, p<0.05)$.

Adding postmotivational factors significantly increased the association with adherence, from $46 \%$ to $56 \%\left(\chi^{2}=11.2, p<0.05\right)$. Postmotivational factors partly mediated the association between motivational factors and adherence. As a result, after including postmotivational factors, none of the motivational factors showed significant partial association with adherence. However, the premotivational factors other than personal relevance continued to have strong associations with adherence after postmotivational factors had been included. The only postmotivational factor with a significant partial association with adherence was the use of the intervention card $(B=1.13, p<0.01)$. The level of innovativeness $(B=0.86, p<0.05)$ and level of formalization ( $B=0.81, p<0.05)$ were still significantly associated with adherence after all other variables had been included in the model.

\subsection{Discussion}

As regards the first goal of our study, we found that more than $90 \%$ of the midwives consistently asked their clients about their smoking behavior, advised their smoking clients to quit smoking, assessed their motivation to quit and discussed the reasons to quit. In line with previous research (Bakker et al., 2003, Cooke et al.,1996), the steps that turned out to be more difficult to implement consistently in daily practice were discussing the barriers of smoking cessation, setting a quit date with motivated clients and providing follow-up care. What is particularly remarkable, because of the simplicity of the action, is that half of the midwives did not provide the clients who quit smoking during pregnancy with the information booklet about relapse prevention. One reason for this may be that the booklets were not always available in the practice, as was reported by $25 \%$ of the midwives. Another reason may be that midwives simply forgot to present the booklet. Almost half of the midwives failed to consistently discuss smoking cessation aids. This might relate to uncertainty about the safety of NRT use in pregnant and 
breastfeeding women (Dempsey \& Benowitz, 2001). The lowest perceived skills were reported for the actions that were least frequently performed, indicating that perceived lack of skills impedes action.

As regards our second study goal, we found that adherent and nonadherent users of the MIS differed significantly on all measured factors in the I-Change model, except for barriers. Within these factors, however, there were several variables for which we did not find significant differences.

With respect to premotivational factors, adherent users were generally more innovative and worked in organizations with higher levels of formalization than nonadherent users. These findings are in line with Rogers' Diffusion theory (Rogers, 1995). Not surprisingly, adherent users were more aware of the problem of smoking among pregnant women, had more knowledge about the MIS and assigned greater personal relevance to smoking cessation guidance. However, adherent users did not perceive more risks of smoking and were not less satisfied with the smoking cessation support they had provided before the MIS was introduced. It is likely that theories of risk perception which are based on the anticipated personal vulnerability related to a health behavior (Weinstein, 1988) are not applicable in this case, because the risks of smoking do not concern the health professionals who use the protocol themselves, but their clients. Dissatisfaction may serve as a cue to action in the decision to use the MIS, but after that it no longer seems to be important.

With respect to motivational factors, adherent users perceived much more social support and had a more positive attitude towards using the MIS, in line with assumptions and findings from social cognitive theories (Ajzen, 1991; Bandura, 1986). However, they did not perceive stronger social norms, and self-efficacy was only higher in stressful situations. In general, self-efficacy was quite low in both adherent and nonadherent users. This implies that additional training facilities may be needed for both groups to facilitate use of the MIS in practice. Another explanation may be that adherent users might be more aware and strict concerning the implications of using the MIS protocol and therefore report low self-efficacy. Since the social norms for both groups were quite positive with respect to using the MIS and the difference between the groups was borderline significant $(p=0.06)$, we assume a positive relation between perceived positive social norms and adherence.

Lastly, with respect to postmotivational factors adherent users perceived themselves as more skilled, and were more likely to perform goal actions like use of the intervention card, and consulting colleagues in case of problems in applying the MIS. Among the five variables with the highest correlations with adherence, there were two postmotivational factors, i.e. skills and intervention card use, two premotivational 
factors, i.e. personal relevance and innovativeness, and only one motivational factor, i.e. social support.

As regards our secondary goal, i.e. assessing the applicability of the I-Change Model in this type of implementation research among midwives, the study found that the factors in the I-Change Model were associated with $56 \%$ of the variance in adherence between midwives, which is a substantial fraction. However, unlike what is assumed in the I-Change Model, the premotivational factors were hardly found to be mediated by motivational factors. A reason for this might be that we only found weak correlations between most motivational factors and adherence. They also provided little additional explanation of the variance to the premotivational factors. Another more general explanation might be related to the cross-sectional design of the study. As assumed in the I-Change Model the present associations between motivational factors and adherence were partly mediated by postmotivational factors. A reason for the limited influence of motivational factors might be that at the point where people have too many tasks to perform for which they are all motivated, factors other than motivation probably become decisive in setting priorities. These factors are for example the postmotivational factors that we found to be very important for adherence. Because of their broad job description and wide interest, midwives are likely to be obliged to set priorities.

Our study was subject to certain limitations. First, the study yielded only crosssectional data, precluding causal inferences. Second, the participation rate was 55\%. Despite our efforts to stimulate all midwives to fill in our questionnaire and despite the fact that we stressed the importance of responding even when they were not using or not accurately using the MIS, we cannot exclude the possibility of selection bias. This may have caused us to overrate the adherence to the MIS, but it is not likely that it affected the results we found on determinants of adherence. Third, despite our preventive efforts, the midwives may have provided biased reports for reasons of social desirability, which may have caused us to overrate the adherence to the MIS, as well as the pros of the protocol.

\section{Implications for research}

Although the I-Change Model provides us with a good starting point to identify potential determinants of adherence, mediating pathways need to be verified in a longitudinal study. It might be a suggestion, when studying protocol adherence, to use a 'direct-path' model that focuses less on motivational factors, since they tend to play only a minor role. As up till now there is no comprehensive model on the importance of postmotivational factors for behavior we recommend exploration of postmotivational factors in further depth. For example to identify the underlying constructs that explain 
why the use of an intervention card is so important for adherence. Unreasoned behavior processes like habits and automaticity might play a role.

\subsection{Conclusions}

The study has provided important insights into the level of adherence by midwives to a smoking cessation guidance protocol and into factors that influence the adherence to a protocol for smoking cessation guidance in midwifery practices. As it is likely that nonadherence negatively affects the effectiveness of smoking cessation protocols, these insights could be used to increase cessation rates by improving implementation. Implementation of the MIS and other smoking cessation interventions in midwifery practices requires stimulation and improvement of midwives' efforts in terms of followup care, discussing barriers of smoking cessation with their clients and setting quit dates with motivated clients. Midwives' skills to engage in these behaviors should be improved, e.g., by providing them with improved training on counseling techniques.

Implementation strategies for future smoking cessation programmes should stimulate consistent use of intervention cards, that serve as a registration system, as a schematic summary of the protocol and as a reminder to implement all actions. Implementation strategies should take the level of formalization of midwifery practices into account, for example by providing midwives who work in less formal settings with extra information on how they can organize and structure the intervention in their practice. Furthermore, strategies should try to increase perceived support, for example by involving the social networks of midwifery practices in the implementation process. 


\section{CHAPTER 6}

\section{Determinants of Midwifery Practices' \\ Implementation of an Intervention for Smoking \\ Cessation Support by Midwives}

Change has a considerable psychological impact on the human mind. To the fearful it is threatening because it means that things may get worse.

To the hopeful it is encouraging because things may get better. To the confident it is inspiring because the challenge exists to make things better 


\section{Abstract}

Implementation of potentially effective health promotion interventions often fails. This longitudinal study aimed to find out which organizational and psychosocial factors predicted whether midwifery practices started to implement a smoking cessation intervention one year later, and which predictors were most important. Data from a cross-sectional study among all 446 Dutch midwifery practices, of whom 251 (56\%) responded, was combined with data on implementation by practices one year later. The results show that implementation occurred more likely in practices with more knowledge on the intervention, with stronger intentions to implement it, with greater dissatisfaction with the way of working at baseline and in larger practices. These factors together predicted $31 \%$ of the variance in implementation. Strategies to increase the start of implementation should mainly focus on increasing knowledge about the intervention and on stressing the disadvantages of the old working methods, using educational activities supplied in easily accessible and interactive educational meetings.

\section{Submitted as:}

Segaar, D., Bolman, C., Willemsen, M.C., De Vries, H. Determinants of midwifery practices' implementation of an intervention for smoking cessation support by midwives 


\subsection{I ntroduction}

Smoking during pregnancy is associated with serious health consequences, such as an increased risk of preterm delivery and infant deaths (US Department of Health and Services, 2004; British Medical Association, 2004). For this reason, pregnant smokers are strongly recommended to quit smoking and guidelines for treatment of tobacco addiction recommend health care professionals to provide pregnant smokers with short smoking cessation interventions based on the Ask, Advice, Assess, Assist and Arrange (5 A's) Model (Raw, McNeill, \& West, 1999; Van Weel et al., 2005).

In the Netherlands, midwives are the main source of information on pregnancyrelated topics. Midwives assist 75\% of all pregnancies. From their third month of pregnancy, women visit their midwives regularly, at least once a month. Thus, midwives are regarded as an excellent channel for providing pregnant women with smoking cessation assistance, and a smoking cessation and relapse prevention protocol based on the 5 A's, called minimal intervention strategy (MIS) for midwives, has been developed for them. When tested in a real-life context, the protocol was found to be more effective than usual care in achieving smoking cessation six weeks after the intervention (7-day abstinence $19 \%$ vs. $7 \%$ ) and six weeks post-partum (7-day abstinence $21 \%$ vs. $12 \%$ ) (De Vries, Bakker, Dolan-Mullen, \& Van Breukelen, in press).

Effective smoking cessation interventions, including those provided during pregnancy, usually do not become part of the daily practice of care providers automatically, and in many cases this does not happen at all (Glasgow, Lichtenstein, \& Marcus, 2003; Haines \& Donald, 1998). Therefore, a broad range of diffusion strategies has been developed and tested for effectiveness and efficiency (Bero et al., 1998; Grimshaw et al., 2004). Several effective diffusion strategies exist. For example, interactive educational meetings can significantly increase knowledge and skills (Davis et al., 1999; Thomson O'Brien et al., 2001), and audit and feedback by providing professionals with information on their own behavior in comparison with that of their peers can influence and improve the behavior (Jamtvedt, Young, Kristoffersen, O'Brien, \& Oxman, 2006). However, the effectiveness of the strategies differs greatly between settings and interventions, and they only seem to work if they target the right barriers (Hulscher, Wensing, Van der Weijden, \& Grol, 2003). Choosing the right strategy to diffuse smoking cessation interventions for pregnant women among midwives requires detailed knowledge of the determinants of this diffusion.

Three stages can be distinguished in the diffusion process of new interventions (innovations): adoption, implementation, and maintenance (Rogers, 1995). Diffusion strategies that target the main determinants of successful diffusion and take the main barriers into account are assumed to improve diffusion and so increase appropriate use (Grimshaw et al., 2004). 
Diffusion of innovations can be seen as changing the behavior of a large group of individuals. The process has been studied by various disciplines, ranging from marketing to psychology, and various theories have been applied to identify factors that may influence successful diffusion (Grol \& Wensing, 2001), such as the theory of planned behavior (Ajzen, 1991, 1995), the social-cognitive theory (Bandura, 1986), the innovation-diffusion theory (Rogers, 1995), and theories about characteristics of innovative organizations (Damanpour, 1991). Studies based on these theories have identified a wide variety of factors that influence diffusion, such as the size of the organization (Castle, 2001; Moch \& Morse, 1977) and individual beliefs about the consequences of the use of the intervention (Michie, Hendy, Smith, \& Adshead, 2004). However, previous studies have failed to simultaneously assess the relative importance of various distinctive factors, such as organizational and psychosocial variables, and have mostly been cross-sectional in nature. In addition, most of them did not study health promotion innovations and were performed outside the field of public health, while information on the generalizability of study findings to other settings and other interventions has often been lacking (Foy, Eccles, \& Grimshaw, 2001; Glasgow et al., 2003).

Few studies have been conducted on the diffusion of smoking cessation interventions among midwives. A study by Cooke (Cooke, 2000) indicated that centralization of decision-making, dissemination strategy, professional status, and working in a clinic at the time of the initial program dissemination were significant predictors of program awareness, adoption, and maintenance in antenatal clinics. Structural variables, such as policy and formalization of rules, were especially found to influence the early dissemination processes. However, since Dutch midwives are not connected to hospitals, it is unclear to what extent these results can be generalized to midwifery care in the Netherlands, which is mainly performed in small practices. Bakker and colleagues (Bakker, Mullen, De Vries, \& Van Breukelen, 2003) concluded that implementation of the MIS protocol in midwifery care was feasible, but did not study determinants of adoption or implementation.

Nevertheless, in the early months of 2002, the Dutch expert centre on tobacco control (STIVORO), together with the Dutch Professional Organization for Midwives (KNOV) started the nationwide dissemination of the MIS. A multifaceted strategy was used. A pilot study into the predictors of whether smoking cessation counseling was perceived as a midwives' task provided an overview of the characteristics of midwives. Important focal points identified were workload, relations with clients, self-efficacy, and practice organization (Bakker, De Vries, Mullen, \& Kok, 2005). To inform all midwives about the existence of the MIS, a letter signed by STIVORO and KNOV and a leaflet were sent to all midwifery practices. Besides contact information, they contained information 
on the goal and content of the MIS and offered a free 3-hour skills training course in their own region. After having completed the training course, the midwives could order relevant materials such as manuals and information magazines for pregnant women. The start of the nationwide MIS introduction was publicized by means of a press release and a public presentation. During the dissemination period, information on the content and goals of the MIS and on contacts for more information on the MIS was disseminated through various channels, such as direct mail, professional journals, and conferences (Kans, 2004).

As determinants of diffusion seem to differ between diffusion stages (Zaltman, Duncan, \& Holbek, 1973; Rogers, 1995; Cooke, 2000; Orlandi, 1987) we performed a series of studies that focused on successive stages of diffusion of the MIS. A crosssectional study on adoption of the MIS within midwifery practices showed that adoption was strongly associated with awareness factors (i.e. knowledge and personal relevance) and motivation factors (i.e. attitudes, social influences and self-efficacy), and to a small extent with some predisposing factors (i.e. availability of practice assistants and proportion of clients of foreign ethnic origin) (Segaar, Bolman, Willemsen, \& De Vries, 2006). A cross-sectional study on successful implementation showed that adherence with the MIS protocol by midwives was strongest associated with some ability factors (i.e. skills and intervention card use), with some awareness factors (i.e. knowledge and personal relevance) and with some motivation factors (i.e. social support, attitude and self-efficacy in stressful situations). Furthermore, the predisposing factors level of innovativeness and level of formalization were strongly related to adherence (Segaar, Bolman, Willemsen, \& De Vries, submitted).

The present study uses a longitudinal design to detect which factors predicted the start of the implementation of the protocol in midwifery practices. Like the abovementioned cross-sectional studies, the present study used the Integrated Change Model (De Vries et al., 2005; De Vries, Mudde, Leijs et al., 2003; De Vries, Mesters, Van 't Riet et al., 2006) as a framework to identify possible determinants of implementation of the MIS. This model builds on an earlier model referred to as the Attitude - Social Influence - Self Efficacy (ASE) model, and integrates concepts of various other social and cognitive models (Bandura, 1986; Ajzen, 1991; Prochaska \& DiClemente, 1983; Weinstein, 1988). The model is presented in Figure 1. 


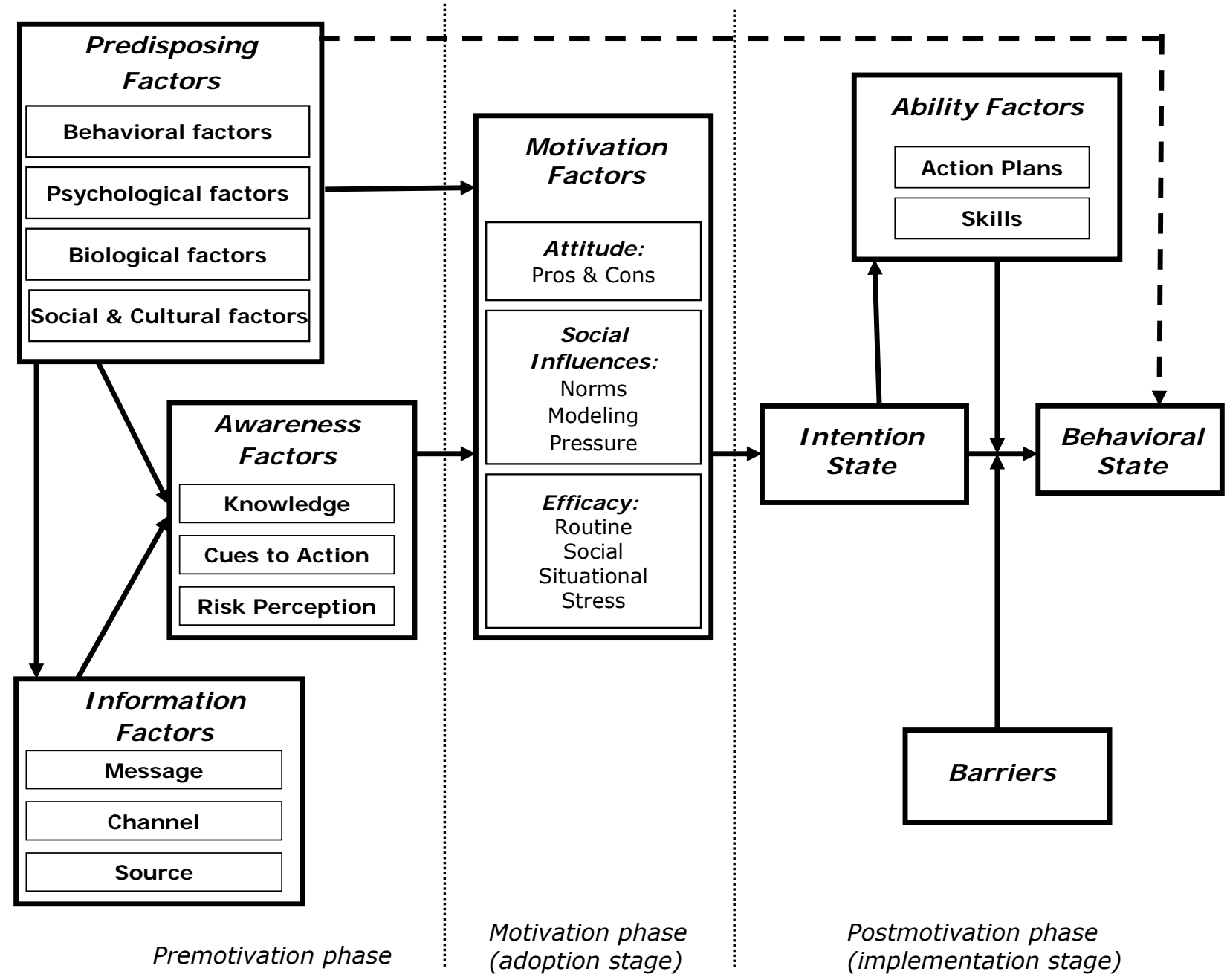

Figure 1. The Integrated Change Model (De Vries et al., 2005, 2006; De Vries, Mudde, Leijs et al., 2003)

The I-Change Model distinguishes three phases in behavioral change processes: the pre-motivation phase, in which a person becomes aware of a problem and the need for a new behavior or a change in an existing behavior (with knowledge, risk perceptions, and cues to action assumed to be important); the motivation phase, in which the intention to perform the new behavior is formed (with attitudes, social influences, and self-efficacy assumed to be important); and the post-motivation phase, in which intention is transformed into behavior (with action plans, skills, and barriers assumed to be important). As adoption of an intervention can be regarded as the positive intention to use it, we considered the adoption stage to be comparable to the motivation phase. Since the implementation of a protocol is the stage that targets the actual use of a protocol that one has decided on using before, we considered the implementation stage to be analogous to the post-motivation phase.

The application of the I-Change Model in research on the diffusion of the MIS has been described more extensively elsewhere (Segaar et al., 2006). The present study 
included the same factors as this cross-sectional study on adoption, but also included additional factors that either could not be included in the cross-sectional analyses, viz. intention and baseline satisfaction, or related to the post-motivation phase, viz. action plans (Gollwitzer \& Brandstätter, 1997; De Vries et al., in press; De Vries, Mesters, Van 't Riet, Willems, Reubsaet, 2006). Information from the present study can help to select practices and factors that should be targeted more intensely by diffusion strategies, which in turn should increase successful implementation (Bartholomew, Parcel, \& Kok, 1998).

\subsection{Methods}

\subsubsection{Study design and procedure}

About two years after the dissemination of the MIS started, in December 2003 (T1), one written questionnaire on the adoption of the MIS and on variables in the I-Change model was mailed to all midwifery practices $(\mathrm{N}=446)$ in the Netherlands, with the request to have it filled out by one of the midwives as a representative of the practice. A letter explaining the aim of the study and a postage-paid return envelope were enclosed. More information about the content of the questionnaire is provided below. Addresses were provided by the Dutch Professional Organization for Midwives KNOV. Reminders consisting of a letter and a copy of the questionnaire were sent to non-responders three weeks later. As an incentive, ten $€ 25$ value coupons were raffled among the midwives who returned the questionnaire. Representatives of 251 (56\%) of the midwifery practices completed and returned the questionnaire. Of these practices, 91 (36\%) were already using the MIS at that time, while 160 (64\%) were not using the MIS yet. One year later, in December 2004 (T2), we assessed whether the 160 non-using practices had started to use the MIS in the meantime. This information was gathered from an Electronic database kept by STIVORO. STIVORO kept this database as it was charged with providing the skills training courses and educational materials for the MIS.

The practices that had started implementing the MIS between December 2003 and December 2004 were compared with the practices that had not started implementation of the MIS, in terms of the variables in the I-Change model.

\subsubsection{Outcome variable}

The outcome variable was whether midwifery practices that did not use the MIS at time 1 $(n=160)$ were implementers of the MIS one year later (time 2$)$ or not. The practices that had not started to implement the MIS at T2 are referred to below as non-implementers, while those that had started the implementation process are referred to as implementers. Practices were considered to be implementers at time 2 if they met two conditions; at least one midwife in the practice had to have been trained in the use of the MIS, and 
after the training, the practice had to have ordered the additional educational materials necessary to administer the MIS, of which a sample set was provided during the training course. This information was gathered from the STIVORO database.

\subsubsection{Independent variables}

All independent variables were measured by means of a questionnaire at T1, consisting of 113 questions, with pre-structured answering options, mostly Likert scales. Factors measured in the questionnaire were derived from the I-Change Model (Figure 1) and the questions were based on existing questionnaires or scales, and on information from literature reviews and earlier work (Bakker et al., 2005; Bolman, De Vries, \& Mesters, 2002; Cooke, 2000; Mesters \& Meertens, 1999; Steckler, Goodman, McLeroy, Davis, \& Koch, 1992). A brief overview of the constructs is shown in Table 1.

Factor analyses and reliability analyses (Cronbach's a) were performed to construct the scales used to model the implementation initiation. Table 1 presents the Cronbach's a values and the number of items per scale. Constructs that need some further explanation are described below.

The predisposing factor of "decision-making style" was measured by five concepts: centralization (decisions entirely made by the individual midwives or centralized at practice level); formalization of decision-making in the practice (using the midwives' own solutions or following set procedures); the amount of information gathered before decisions are made; the proportion of external information sources versus internal information sources consulted for decisions; and the way disagreements are solved. With respect to information factors, midwives were asked if they had received information from the five most important information sources on the MIS (see Table 1).

With respect to awareness factors, knowledge was measured by four questions (yes/no): "Have you ever heard of the MIS?", "Do you know what the MIS is?", "Do you know how the MIS works?", and "Do you know precisely what the implications for a midwife are if she uses the MIS?". An index score was calculated by summing the number of questions a respondent answered yes to. Risk perception was assessed by asking two questions about perceived risks from smoking for the baby. Cues to action were measured by two scales: personal relevance and satisfaction. Personal relevance was measured with four items relating to the perceived importance of smoking cessation support given to pregnant women. Satisfaction referred to the extent to which a person was satisfied with the way smoking cessation guidance was organized in their practice at T1, i.e. before the MIS had been implemented. 
Table 1. Variables included in the analyses

\begin{tabular}{|c|c|c|c|}
\hline Concept & Variable & $\begin{array}{c}\mathrm{N} \\
\text { questions }\end{array}$ & Scale \\
\hline \multicolumn{4}{|l|}{ Predisposing factors } \\
\hline \multirow[t]{2}{*}{ Biological factors } & Gender & 1 & \\
\hline & Age (years) & 1 & \\
\hline \multirow[t]{2}{*}{ Behavioral factors } & Smoker (yes/no) & 1 & \\
\hline & Years working as a midwife $(N)$ & 1 & \\
\hline \multicolumn{4}{|c|}{ Social and cultural factors } \\
\hline \multirow[t]{2}{*}{ - Environment } & Clients of non-Dutch origin (\%) & 1 & \\
\hline & Clients with a low educational level (\%) & 1 & \\
\hline \multirow[t]{6}{*}{ - Organization } & Number of midwives per practice $(N)$ & 1 & \\
\hline & Practice assistants available (yes/no) & 1 & \\
\hline & Number of pregnancies monitored per year $(N)$ & 1 & \\
\hline & Time available for the first visit ( $N$ minutes) & 1 & \\
\hline & Practice innovativeness $(-2 /+2)$ & 1 & \\
\hline & Workload $(-3 /+3)$ & 1 & \\
\hline -Decision-making & Centralization $(0 / 4)$ & 1 & \\
\hline \multirow[t]{5}{*}{ styles } & Formalization $(0 / 4)$ & 1 & \\
\hline & Level of disagreement $(0 / 4)$ & 1 & \\
\hline & Disagreements solved by discussion (yes/no) & 1 & \\
\hline & Amount of information used $(0 / 4)$ & 1 & \\
\hline & External or internal information sources $(0 / 4)$ & 1 & \\
\hline \multirow[t]{5}{*}{ I nformation factors } & Dutch national association of midwives (\% yes) & 1 & \\
\hline & Dutch expert center on tobacco control (\% yes) & 1 & \\
\hline & National journal for midwives (\% yes) & 1 & \\
\hline & Colleagues (\% yes) & 1 & \\
\hline & Midwifery schools (\% yes) & 1 & \\
\hline \multicolumn{4}{|l|}{ Awareness factors } \\
\hline Knowledge & Knowledge level (yes/no) & 4 & index \\
\hline \multirow[t]{2}{*}{ Cues to action } & Personal relevance (agreement $-2 /+2$ ) & 4 & $a=0.64$ \\
\hline & Satisfaction (agreement $-2 /+2$ ) & 3 & $a=0.82$ \\
\hline Risk perception & Risk perception ( $1=$ no risk $/ 10=$ very high risk) & 2 & $r=0.55$ \\
\hline \multicolumn{4}{|l|}{ Motivation factors } \\
\hline \multirow[t]{3}{*}{ Attitude items } & Advantages scale (agreement $-2 /+2$ ) & 15 & $a=0.88$ \\
\hline & Disadvantages scale (agreement $-2 /+2$ ) & 10 & $a=0.72$ \\
\hline & Ambivalence (agreement $-2 /+2$ ) & 2 & $r=0.74$ \\
\hline \multirow[t]{3}{*}{ Social influences } & Perceived norm $(-2=$ negative,$+2=$ positive $)$ & 6 & $a=0.87$ \\
\hline & Perceived support $(-2=$ discouragement, $+2=$ support $)$ & 6 & $a=0.86$ \\
\hline & Modeling (\% others using the MIS) & 1 & \\
\hline \multirow[t]{2}{*}{ Self-efficacy } & $\begin{array}{l}\text { Situation-specific self-efficacy }(-3=\text { will definitely not } \\
\text { succeed, }+3=\text { will definitely succeed })\end{array}$ & 15 & $a=0.84$ \\
\hline & Self-efficacy in general: support $>50 \%$ of clients? $(-3 /+3)$ & 1 & \\
\hline I ntention state & Intention $(-2=$ definitely not $/+2=$ definitely $)$ & 1 & \\
\hline Ability factors & Action plans $(-2=$ definitely not $/+2=$ definitely $)$ & 5 & $a=0.90$ \\
\hline
\end{tabular}

With respect to motivation factors, attitude was assessed by three constructs: pros, cons, and attitudinal ambivalence (Ajzen, 2001). Pros and cons about using the MIS were assessed by 25 questions, including 11 items on beliefs about the intervention characteristics (Bolman et al., 2002; Mesters \& Meertens, 1999; Pieterse, 2001; Rogers, 2002; Steckler et al., 1992) and 14 items on perceived health benefits and other outcome expectations (Bandura, 1986) of using the MIS protocol (Bakker et al., 2003). A 
factor analysis with oblimin rotation on the attitude variables revealed two attitude factors, viz., the pros and the cons. Perceived support and norms were assessed for six subgroups, viz. general practitioners, gynecologists, other midwives, pregnant women, Dutch Professional Organization for Midwives, the national organization for midwives. Situation-specific self-efficacy expectations were measured by asking midwives whether they would succeed in providing smoking cessation assistance with the MIS in 15 situations occurring in daily practice, e.g. if a client resists talking about smoking, if a client has a low educational level, if consulting hours are running late. Situations were derived from previous research on smoking cessation support in midwifery care (Bakker et al., 2003; Cooke, Mattick, \& Campbell, 2000). A self-efficacy scale was calculated by averaging the scores for the 15 situations.

Intention was measured by one question: "Does your practice intend to use the MIS?", rated on a 5 -point scale ranging from $-2=$ no definitely not, to $+2=y e s$ definitely. Action plans referred to specific intentions with respect to relevant strategies that would enable individuals to attain the final goal (Locke \& Latham, 2002), which in this study was the implementation of the MIS. Five questions were asked, for example: "Do you intend to talk to colleagues about the MIS?" and "Do you intend to attend a training course on using the MIS?".

\subsubsection{Statistical analyses}

Differences between non-implementers and implementers were tested with t-tests for continuous variables or Chi-square tests for dichotomous variables.

To estimate the strength of the relationship between the prediction variables (T1) and implementation (T2), correlations ( $r$ ) were computed for the variables that differed significantly between the two groups according to the t-test or Chi-square test. Phi correlations were calculated for dichotomous variables, and point-biserial correlations for continuous variables. Both are special cases of the Pearson correlation.

To identify the main predictors of implementation and to check on the mediating pathways defined in the I-Change Model, five logistic regression analyses were performed, using backward likelihood ratio procedures $\left(p_{\text {in }}=0.05 ; p_{\text {out }}=0.10\right)$. The dependent variable in all regression analyses was implementation of the MIS (yes/no) at T2. In each successive analysis, an additional group of independent variables (measured at T1) was included in the regression model, following the pathway identified by the IChange Model from left to right. The first analysis included all predisposing and information factors as independent variables. The second analysis included all predisposing, information, and awareness factors. The third analysis included the above factors and added motivation factors. Intention was added in the fourth analysis, and the final regression analysis comprised all factors, including action plans. Because of the 
large number of variables relative to the number of respondents, we were not able to include all variables in the regression analysis, so our analysis included only variables with $\mathrm{p}<0.20$ in the t-test or Chi-square test (Tabachnick \& Fidell, 2001). Standardized zscores of the variables were used to compare the magnitude of the partial association between variables and implementation, based on their standardized beta values $(\beta)$. Nagelkerke's $\mathrm{R}^{2}$ was used to approximate the total predictive value of the most important variables. Assumptions for regression analysis were met, and checks on multicollinearity between the independent variables were found to be satisfactory. All analyses were performed with SPSS version 14.0. Differences were considered significant when $p<0.05$

\subsection{Results}

\subsubsection{Sample description}

Of the 160 midwifery practices that had not adopted the MIS when filling out the questionnaire at T1, 45 (28\%) had initiated the implementation of the MIS within one year, whereas $115(72 \%)$ had not. Ninety-seven percent of the practice representatives were female and the mean age was $36(S D=10)$ years. They had been working as a midwife for an average of 10 years $(S D=9)$. Seven percent $(n=11)$ smoked.

\subsubsection{Differences between implementing and non-implementing practices}

The mean scores of implementers and non-implementers on the predisposing and information factors at $\mathrm{T} 1$ are shown in Table 2, while Table 3 presents the mean scale scores on awareness factors, motivation factors, intention, and action plans at T1. Implementers and non-implementers hardly differed in terms of the pre-motivation factors (i.e. predisposing and information factors), but many differences were found with respect to motivation and post-motivation factors.

\section{Predisposing factors and information factors}

Table 2 shows that implementing and non-implementing practices at T2 hardly differed in terms of the predisposing factors assessed at T1. Only practice size mattered, in that implementing practices were significantly larger than non-implementing practices (3.8 vs. 3.1 midwives per practice). Table 2 also shows that there were no significant differences between implementers and non-implementers as regards the information factors assessed at T1. Although implementers were more likely to have been informed about the MIS by the Dutch national association of midwives, this relation was borderline non-significant $(p=0.07)$. 
Table 2. Means of and significant differences in predisposing factors and information factors for non-implementers and implementers ${ }^{1}$

\begin{tabular}{|c|c|c|c|c|}
\hline Variable (scale) & $\begin{array}{l}\text { Overall } \\
(N=160)\end{array}$ & $\begin{array}{c}\text { Non- } \\
\text { implementers } \\
(n=115)\end{array}$ & $\begin{array}{l}\text { Implementers } \\
\quad(n=45)\end{array}$ & $\begin{array}{l}\mathrm{p} \text {-value } \\
\mathrm{chi}^{2} / \text { t-test }\end{array}$ \\
\hline \multicolumn{5}{|l|}{ Biological factors } \\
\hline Gender (\% female) $)^{\#}$ & 97 & 97 & 98 & \\
\hline Age in years & 36 & 36 & 34 & \\
\hline \multicolumn{5}{|l|}{ Behavioral factors } \\
\hline Smokers (\% yes) ${ }^{\#}$ & 7 & 9 & 2 & \\
\hline Years working as a midwife & 10 & 11 & 9 & \\
\hline \multicolumn{5}{|l|}{ Social and cultural factors } \\
\hline \multicolumn{5}{|l|}{ Environment } \\
\hline Clients of non-Dutch origin (\%) & 18 & 18 & 16 & \\
\hline Clients with a low educational level (\%) & 41 & 42 & 39 & \\
\hline \multicolumn{5}{|l|}{ Organization } \\
\hline Number of midwives per practice & 3.3 & 3.1 & 3.8 & $\mathrm{p}<0.05$ \\
\hline Practices with assistants (\% yes) ${ }^{\#}$ & 46 & 45 & 49 & \\
\hline Number of pregnancies monitored per year & 325 & 317 & 344 & \\
\hline Time available for the first visit, in minutes & 33 & 33 & 34 & \\
\hline Level of innovativeness of practice & 1.03 & 1.00 & 1.11 & \\
\hline $\begin{array}{l}\text { Workload } \\
\text { Decision-making styles }\end{array}$ & 1.05 & 1.11 & 0.91 & \\
\hline Centralization level & 2.74 & 2.67 & 2.93 & \\
\hline Formalization level & 2.17 & 2.16 & 2.21 & \\
\hline Level of disagreement & 0.30 & 0.26 & 0.39 & \\
\hline Disagreements solved by discussion (\% yes) ${ }^{\#}$ & 37 & 35 & 44 & \\
\hline Amount of information used & 3.80 & 3.80 & 3.78 & \\
\hline $\begin{array}{l}\text { Number of external information sources versus } \\
\text { internal sources }\end{array}$ & 2.08 & 2.10 & 2.02 & \\
\hline \multicolumn{5}{|l|}{ I nformation factors $(\% \text { yes) })^{\#}$} \\
\hline Dutch national association of midwives & 53 & 49 & 64 & \\
\hline Dutch expert center on tobacco control & 46 & 44 & 48 & \\
\hline National journal for midwives & 39 & 39 & 38 & \\
\hline Colleagues & 18 & 17 & 20 & \\
\hline Midwifery schools & 8 & 8 & 7 & \\
\hline
\end{tabular}

${ }^{1}$ The value in table is the mean, unless indicated otherwise ${ }^{*} r$ is only presented if significant ${ }^{*}$ Differences between the groups tested with chi-square tests

\section{Awareness factors}

Table 3 shows that implementers had significantly more knowledge about the MIS at T1 than non-implementers. With respect to personal relevance, implementers found the provision of smoking cessation assistance, both in general and specifically by midwifery practices, more important than non-implementers. They also attached greater importance to smoking cessation by pregnant women than non-implementers did. Furthermore, implementers were much less satisfied with the type of assistance that the practice had been giving to pregnant women at T1 than non-implementing practices. Both implementers and non-implementers perceived very high risks from smoking during 
pregnancy for the baby's health before and after its birth (scale mean $=8.7$ on a 10 -point scale); they did not differ significantly in this respect.

\section{Motivation factors}

Implementers and non-implementers differed with regard to many motivation factors at T1 (see Table 3). Practices where midwives perceived many advantages at T1 were more likely to be implementers at $\mathrm{T} 2$ than practices where midwives perceived fewer advantages. Implementers and non-implementers did not differ significantly in terms of the perceived disadvantages scale.

As Table 3 shows, both implementers and non-implementers perceived positive social norms and social support, but implementers perceived significantly more positive norms towards using the MIS in the practice and more support from their social environment than non-implementers did. The perceived percentage of other midwives using the MIS (modeling) did not differ significantly between implementers and nonimplementers.

Table 3. Means of and significant differences between non-implementers and implementers in awareness and motivation factors, action plans and intention state

\begin{tabular}{|c|c|c|c|c|}
\hline Variable (scale) & $\begin{array}{l}\text { Overall } \\
(\mathrm{N}=160)\end{array}$ & $\begin{array}{c}\text { Non- } \\
\text { implementers } \\
(n=115)\end{array}$ & $\begin{array}{l}\text { Implementers } \\
\quad(n=45)\end{array}$ & $\begin{array}{c}\text { p-value } \\
\text { t-test }\end{array}$ \\
\hline \multicolumn{5}{|l|}{ Awareness factors } \\
\hline Knowledge level & 2.54 & 2.30 & 3.16 & $\mathrm{p}<0.01$ \\
\hline Personal relevance & 0.99 & 0.90 & 1.24 & $p<0.01$ \\
\hline Satisfaction & -0.15 & 0.02 & -0.56 & $\mathrm{p}<0.01$ \\
\hline Risk perception & 8.66 & 8.56 & 8.85 & \\
\hline \multicolumn{5}{|l|}{ Motivation factors } \\
\hline \multicolumn{5}{|l|}{ Attitude } \\
\hline Advantages & 0.34 & 0.26 & 0.52 & $\mathrm{p}<0.01$ \\
\hline Disadvantages & -0.08 & -0.04 & -0.18 & \\
\hline Ambivalence & -0.10 & 0.11 & -0.64 & $\mathrm{p}<0.01$ \\
\hline \multicolumn{5}{|l|}{ Social influence } \\
\hline Perceived norm & 0.58 & 0.46 & 0.88 & $\mathrm{p}<0.01$ \\
\hline Perceived support & 0.51 & 0.47 & 0.63 & $\mathrm{p}<0.05$ \\
\hline Modeling (perceived \% of other midwives using MIS) & 23 & 22 & 26 & \\
\hline \multicolumn{5}{|l|}{ Self-efficacy } \\
\hline Situation-specific self-efficacy & -0.60 & -0.61 & -0.59 & \\
\hline Able to support more than $50 \%$ of smoking clients? & 0.07 & -0.12 & 0.56 & $\mathrm{p}<0.01$ \\
\hline \multicolumn{5}{|l|}{ I ntention } \\
\hline Does your practice intend to use the MIS? & 0.44 & -0.16 & 0.73 & $\mathrm{p}<0.01$ \\
\hline Action plans & 0.41 & 0.25 & 0.81 & $\mathrm{p}<0.01$ \\
\hline
\end{tabular}

$* r$ is only presented if significant

Self-efficacy levels of both implementers and non-implementers in several specific situations were quite low, and the mean situation-specific self-efficacy was not significantly related to implementation of the MIS. General self-efficacy as regards 
supporting at least half of all smoking clients with the MIS was significantly higher among implementers than non-implementers.

\section{Intention and action plans}

Table 3 shows that implementers had stronger intentions to use the MIS than nonimplementers, so practices whose representatives had a strong intention to use the MIS were significantly more likely to have initiated implementation one year later than practices whose representatives had a weak intention. Implementers also had made significantly more action plans than non-implementers.

\subsubsection{Level of association}

Figure 2 presents the Pearson correlation coefficients of the variables that were significantly correlated with implementation. Figure 2 shows, from left to right, that implementation correlated most strongly with positive intentions, followed by low levels of ambivalence, low satisfaction with the existing working methods, high level of knowledge about the existence and content of the MIS, high perceived social norms, perceiving more advantages, making more action plans, high personal relevance, high general self-efficacy, large number of midwives per practice, and high perceived social support.

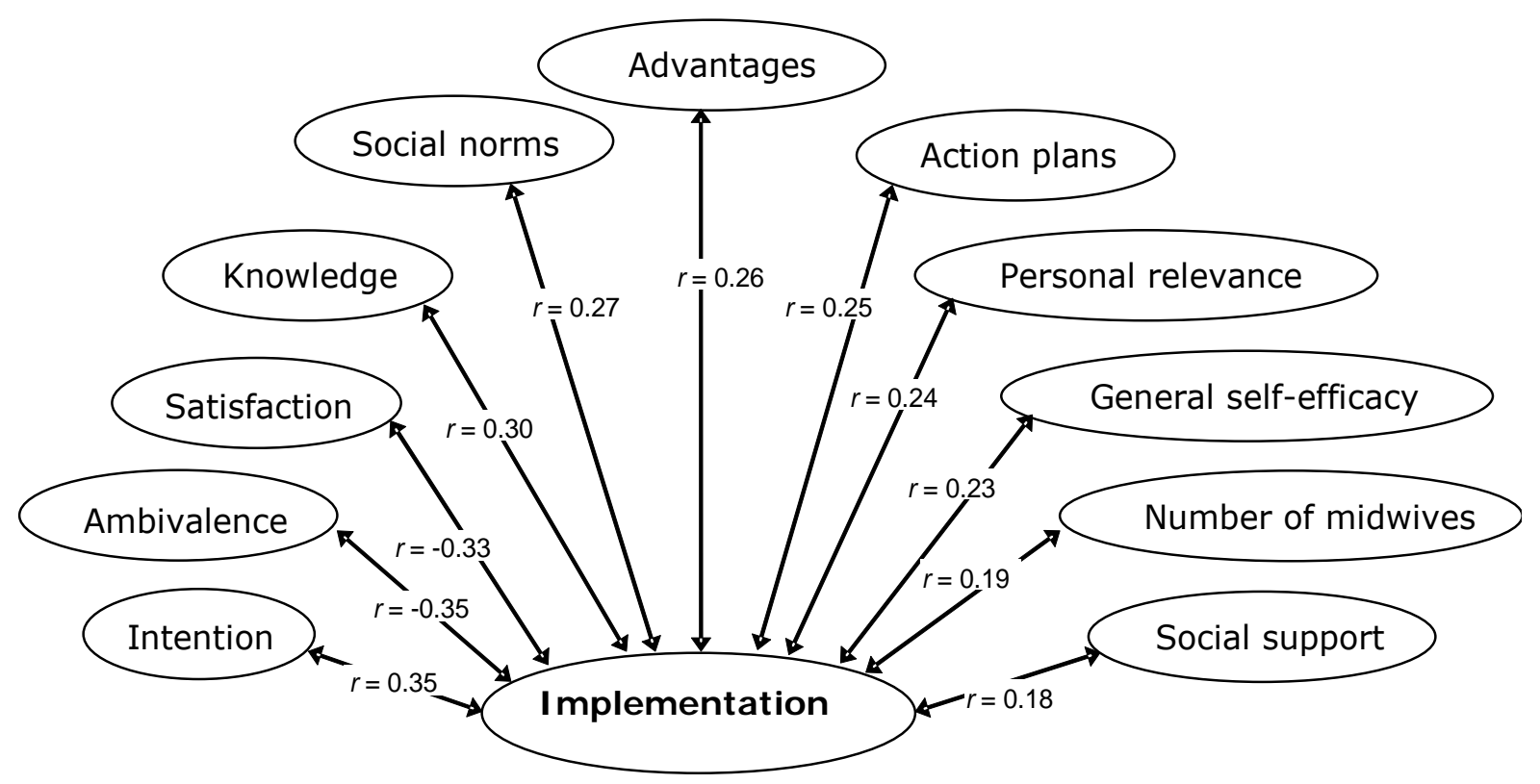

Figure 2. Pearson correlations of the most important factors associated with implementation 


\subsubsection{Predictive value}

Results of the logistic regression analyses are presented in Table 4. The variables presented in the table are those that were left in the predictive model after the backward likelihood procedure had been completed. Logistic regression analysis showed that the predisposing and information factors, represented by only two significant variables (a larger number of midwives in the practice and information received from the professional organization for midwives), predicted $7 \%$ of the variance in implementation. A high level of knowledge about the existence and content of the MIS and low satisfaction with the working methods at T1 was associated with a larger increase in the likelihood of implementation. Together with the number of midwives in the practice, these variables predicted about $27 \%$ of the variance in implementation.

Only one motivation factor, low ambivalence, significantly increased the likelihood of implementation when all significant pre-motivation factors were controlled for. Including ambivalence in the predictive model increased the total predictive value to $29 \%$. Ambivalence mediated the influence of dissatisfaction somewhat, but the unique contribution of satisfaction remained significant. Intention mediated the influence of ambivalence and was a slightly stronger predictor of implementation than ambivalence. The total proportion of variance explained increased to $31 \%$. Action plans did not add any predictive value to these factors. As action plans and intention correlated quite strongly $(r=0.74)$, having made action plans was also added to a model without intention. However, it still did not increase the proportion of explained variance.

Table 4. Summary of the logistic regression analyses for variables predicting initiation of the implementation of the MIS for midwives.

\begin{tabular}{|c|c|c|c|c|c|}
\hline & $\beta$ & SE $(\beta)$ & OR $(95 \%)$ & $\mathrm{p}$-value & $\mathrm{R}^{2}$ \\
\hline Analysis 1 & & & & & 0.074 \\
\hline Number of midwives per practice & 0.32 & 0.19 & $1.37(0.95-1.97)$ & 0.09 & \\
\hline Informed by association of midwives & 0.40 & 0.18 & $1.49(1.05-2.12)$ & 0.03 & \\
\hline Analysis 2 & & & & & 0.265 \\
\hline Number of midwives per practice & 0.34 & 0.19 & $1.40(0.96-2.04)$ & 0.08 & \\
\hline Knowledge & 0.68 & 0.23 & $1.98(1.26-3.11)$ & 0.00 & \\
\hline Satisfaction & -0.78 & 0.22 & $0.46(0.30-0.71)$ & 0.00 & \\
\hline Analysis 3 & & & & & 0.288 \\
\hline Knowledge & 0.60 & 0.23 & $1.82(1.16-2.88)$ & 0.01 & \\
\hline Satisfaction & -0.61 & 0.23 & $0.55(0.35-0.85)$ & 0.01 & \\
\hline Ambivalence & -0.53 & 0.22 & $0.59(0.38-0.91)$ & 0.02 & \\
\hline Analysis $4+5$ & & & & & 0.310 \\
\hline Number of midwives per practice & 0.35 & 0.20 & $1.42(0.96-2.10)$ & 0.08 & \\
\hline Knowledge & 0.64 & 0.23 & $1.90(1.21-3.00)$ & 0.01 & \\
\hline Satisfaction & -0.52 & 0.24 & $0.60(0.37-0.96)$ & 0.03 & \\
\hline Intention & 0.57 & 0.23 & $1.77(1.12-2.81)$ & 0.02 & \\
\hline
\end{tabular}


In sum, the likelihood of implementation at T2 was best predicted by, in order of the strength of partial correlations (high to low): knowledge, intention to implement the MIS, satisfaction with the working methods and practice size at T1.

\subsection{Discussion}

The present study examined determinants of the implementation of a minimal-contact intervention for smoking cessation support (MIS) by midwifery practices in the Netherlands in a longitudinal design. Implementation was assessed one year after the determinants were measured.

The highest likelihood of implementation was found in practices where more knowledge was available about the existence and content of the MIS, where midwives had strong intention to implement the MIS, where they were more dissatisfied with the current working methods, and which had a larger size. These factors together predicted $31 \%$ of the variance in implementation.

There was thus only one predisposing factor that significantly determined implementation: a larger number of midwives per practice significantly increased the likelihood of implementation. This finding is in line with those of other studies that found that the size of the organization increased early implementation (Castle, 2001; Cooke, Mattick, \& Barclay, 1996). However, another study found that practice size was not significantly associated with the adoption of the MIS by midwives (Segaar, Bolman, Willemsen, \& De Vries, in press), while a cross-sectional study found no significant association between practice size and the quality of use of the MIS by individual midwives (Segaar et al., submitted). The positive effect of practice size in the present study can be explained by the fact that at least one midwife in the practice had to attend the skills training course, which, on average, requires less effort per midwife if a practice consists of more midwives.

It is remarkable that having a practice assistant was not significantly related to implementation, because a previous, cross-sectional study found that having a practice assistant was strongly associated with adoption, and was believed to play a role in organizing and administering the MIS (Segaar et al., in press). One reason for the discrepancy might be that the present study did not include the early adopters, because they had already started to use the MIS at baseline, and that these early adopters were the ones with the most enthusiastic and active assistants.

Contrary to our findings, Cooke and colleagues (Cooke, 2000) found that structural and work climate variables, policy and formalization of rules were strongly related to whether midwives in antenatal clinics had started to use a smoking cessation program. Unlike midwifery practices, antenatal clinics are hospital settings, which are larger and more complex organizations. Therefore, the differences in organizational 
characteristics between midwifery practices may be smaller than those between antenatal clinics, which may have decreased the likelihood of finding relations between organizational characteristics and implementation in our study.

With respect to information factors, practices that had received information specifically from the Dutch Professional Organization for midwives were significantly more likely to implement the MIS than those who did not, when all significant predisposing factors were controlled for. This information source had also been found to be positively associated with adoption (Segaar et al., in press). Information from this source was probably read more attentively because this source is highly valued by midwives, as is evident from the fact that $90 \%$ of all midwives are members of this organization (Kenens \& Hingstman, 2004).

As mentioned above, a higher level of knowledge about the MIS, more dissatisfaction with the current working methods and greater personal relevance increased the likelihood of implementation of the MIS within one year. This finding is in line with findings from our previous cross-sectional study (Segaar et al., 2006), and with the diffusion theory, in the sense that knowledge and persuasion (caused by for example dissatisfaction) are the first steps in the innovation-decision process (Rogers, 1995). Knowledge and dissatisfaction significantly increased the likelihood of implementation, even when all other significant factors in the Integrated Change Model were controlled for.

In line with social cognitive theories and various studies based on these theories (McCarty, Hennrikus, Lando, \& Vessey, 2001; Mesters \& Meertens, 1999; Park et al., 2003; Paulussen, Kok, \& Schaalma, 1994), several motivation factors were significantly associated with implementation of the MIS. Greater perceived advantages, social norms and support, and higher perceived self-efficacy to support more than half of the smoking clients with the MIS significantly increased implementation. If the representatives of midwifery practices were more convinced of their opinion about the MIS (low ambivalence), the likelihood of implementation increased significantly, even when controlling for all other significant factors, except intention. This indicates that the practices that do not implement the MIS are ambivalent in their opinion about the pros and the cons of the intervention. There was no significant relation between the perceived disadvantages scale and implementation. Perceived disadvantages were probably mostly solved within the period between the time when disadvantages were assessed (time 1) and the time of implementation, at most one year later. Self-efficacy towards consistently performing the MIS in specific difficult situations was quite low. However, this did not prevent practices from implementing the MIS.

Together with ambivalence, the intention to use the MIS was the variable showing the strongest correlation with implementation. Yet, the correlation between intention and 
implementation was quite low $(r=0.35)$ compared to correlations between intention and behavior found in other studies. Meta-analyses have reported correlations between intention and behavior of around 0.50 (Armitage \& Conner, 2001; Sheeran, 2002). However, these studies often had cross-sectional designs, which tend to result in higher correlations than longitudinal designs. Given the limited association between intention and implementation in our case, it seems to be very important to solve start-up problems associated with the implementation of the MIS. One way to do this might be by formulating specific action plans (Gollwitzer, 1999; Locke \& Latham, 2002). However, we did not find support for this in the present study, which showed that although the likelihood of implementation was higher if practices formulated more action plans than if they did not, the action plans did not contribute to the prediction of implementation in addition to intention. However, this does not mean that actions plans do not play a role in implementation. One reason why we did not find an additional contribution of action plans to the prediction of implementation might be that the action plans as we defined them were too much like sub-intentions instead of specific action-directed plans, such as implementation intentions (Gollwitzer, 1999). But action plans might also play a more important role in determining the success or quality of the implementation, rather than in initiating the implementation.

The present study was subject to some limitations. First, although we invited all midwifery practices to fill out the first questionnaire, $56 \%$ of the practices enrolled. This may have resulted in selection bias. Data from the electronic databases of STIVORO show that $45 \%$ of all Dutch midwifery practices had started implementation of the MIS in December 2004 (T2). Of the 251 respondents in our study at T1, 54\% $(n=136)$ had started implementation of the MIS at T2. This shows that practices that did not implement the MIS were slightly underrepresented in our sample. In view of the low proportion of smokers among the participants in our study (7\%) compared to the proportion of smokers among Dutch women in general (25\% in 2004 (NIPO, 2005)), smoking midwives may have been underrepresented. Even though midwives have been found to smoke less than the general female population (Sacker, 1990) the figure of $7 \%$ is unexpectedly low. This may have caused an underestimation of the effect of smoking on implementation. There is no reason to think that the other results were considerably affected by selection bias. Second, although we used objective measures of the initiation of the MIS, we had no data on the quality of implementation available for this study; the same is true for the speed of implementation, as we only used one time-point for assessment.

Despite these limitations, the study has provided valuable information on relevant factors that determine whether midwifery practices start to implement a smoking cessation intervention. The most important determinants were knowledge about the 
intervention, intention, dissatisfaction with the previous methods used to help clients stop smoking, and practice size. However, a large proportion of the variance in implementation remained unexplained by these determinants. Therefore, we recommend further research in this field, which should include other types of variables. More attention could, for example, be focused on ability factors, such as implementation intentions (Gollwitzer \& Brandstätter, 1997) and skills and attention could be focused on identifying specific barriers.

Practical implications of these findings are that strategies to increase initial implementation of the MIS should mainly focus on increasing knowledge about the MIS, as a convincing message may increase the perceived need for an intervention on smoking cessation support, as well as dissatisfaction with the "old" working methods. Although large-scale didactic education courses are a relatively cheap way to reach a large group of people and increase their knowledge, they hardly increase the use of an intervention without additional measures (Grimshaw et al., 2004). Therefore, in the current phase they should be combined with easily accessibly interactive education meetings, as such sessions have proved to be effective in increasing intervention use by professionals (Davis et al., 1999; Thomson O'Brien et al., 2001). 



\title{
CHAPTER 7
}

\author{
Adoption and Implementation of Smoking \\ Cessation Support in Two Health Care Settings
}

If we want more evidence-based practice, we need more practice-based evidence. 


\begin{abstract}
Diffusion of interventions often fails. This chapter presents data on the importance of a comprehensive set of factors, including organizational, motivational, ability and awareness factors, on the diffusion of a protocol for smoking cessation guidance by healthcare professionals. Data from four studies are used. Comparisons are made between adoption and implementation in two healthcare settings, namely midwifery practices and cardiac wards. Smoking behavior, level of centralization, proportion of external versus internal information sources used and workload were not significantly associated with either adoption or successful implementation in either of the settings. Knowledge, perceived advantages, disadvantages, social norms and pressure were significantly associated with adoption and adherence in both settings. Several other variables were significantly associated with either adoption or successful implementation or in only one of the two settings. In conclusion, the design of diffusion strategies should distinguish between diffusion stages and study the target setting in detail, and should take setting specific characteristics into account to optimize the strategies.
\end{abstract}

\title{
Submitted as:
}

Segaar, D., Bolman, C., Willemsen, M.C., De Vries, H. Adoption and implementation of smoking cessation support in two health care settings 


\subsection{I ntroduction}

Health care interventions, such as guidelines or protocols, are frequently underused and/or inappropriately used (Davis \& Taylor-Vaisey, 1997; Grol, 2001; Katz, Brown, Muehlenbruch, Fiore, \& Baker, 2004) and therefore seldom have the intended health impact. Several international studies suggest that at least one third and probably even more patients do not receive the care they should receive according to scientific evidence or evidence-based guidelines (Schuster, McGlynn, \& Brook, 2005; Seddon, Marshall, Campbell, \& Roland, 2001). The process by which new interventions are transferred to their target population of users is called diffusion and consists of the adoption, implementation and maintenance stages (Rogers, 1995). Diffusion strategies that target the main determinants of successful diffusion and take the main barriers into account are assumed to improve diffusion and so increase appropriate use (Grimshaw et al., 2004).

Diffusion of an intervention in a healthcare setting involves changes in the behavior of many individuals in an organization. This process has been studied by various disciplines, ranging from marketing to psychology, using various theories about factors that have the potential to promote successful diffusion of interventions. These theories can be classified into three groups, according to the type of characteristics they focus on. First, there are theories that focus on organizational factors such as the contingency model of strategic decision-making (Koopman \& Pool, 1991, 1994) and theories on characteristics of innovative organizations (Damanpour, 1991). Studies based on organizational theories have identified a variety of factors that influence diffusion, such as organization size (Castle, 2001; Moch \& Morse, 1977), level of centralization (Damanpour, 1991; Harting et al., 2005) and level of formalization (Zaltman, Duncan, \& Holbek, 1973). Second, there are theories that focus on characteristics of the individual who needs to adopt and use an innovation. In this respect, several social cognitive theories, like the theory of planned behavior, the social cognitive theory and the ASE model, have been used to explain diffusion (Ajzen, 1991, 1995; Green \& Kreuter, 1999; Prochaska \& DiClemente, 1983; Bandura, 1986; Mittman, Tonesk, \& Jacobson, 1992; De Vries, Dijkstra, \& Kuhlman, 1988; De Vries \& Mudde, 1998). These theories assume that behavior is influenced by the intention of an individual to engage in behavior, which is in turn influenced by cognitive perceptions and the influence of the social environment. Studies using these theories have found significant relations between the diffusion of various health promotion interventions and factors like attitude towards the intervention (Fahrenwald \& Walker, 2003; Mesters \& Meertens, 1999; Haagen et al., 2005; Michie, Hendy, Smith, \& Adshead, 2004), perceived social influence (Paulussen, Kok, \& Schaalma, 1994; Wilhelm, 2002) and self-efficacy towards using the intervention (Wilhelm, 2002; Fahrenwald \& Walker, 2003; Haagen et al., 2005). Third, there is a theory related to the social cognitive theories, focusing on intervention characteristics, 
i.e. the diffusion theory (Rogers, 1995). This theory states that successful diffusion is closely related to five characteristics of an intervention, viz. its relative advantage, compatibility, observability, trialability and complexity. As intervention characteristics are assumed to affect diffusion through an individual's perception of the intervention (Rogers, 1995), some social cognitive theories have included these factors as a part of attitude (Bolman, De Vries, \& Mesters, 2002; Paulussen et al., 1994; Bakker, De Vries, Mullen, \& Kok, 2005). All theories described in the above paragraph have so far been applied separately, whereas it would seems necessary to combine them to get a complete overview of factors that are related to diffusion.

Although the large majority of studies on diffusion have been performed in fields other than that of public health, studies of the determinants of diffusion of interventions in healthcare organizations showed that the organizational, individual and intervention characteristics also affect the diffusion of interventions among healthcare organizations. Fleuren and colleagues (Fleuren, Wiefferink, \& Paulussen, 2004) performed a systematic literature search on characteristics affecting the diffusion of interventions among healthcare organizations. Based on the 57 studies they retrieved, they listed 50 determinants that potentially affect the innovation process in healthcare organizations, including the organizational, individual and intervention characteristics mentioned above.

Unfortunately, however, previous studies failed to assess the relative importance of the various factors within a more comprehensive framework, and very few studies have distinguished and compared diffusion stages. Distinguishing between the stages is important, since the studies that did this showed that the relative influence of factors depends on the diffusion stage (Zaltman et al., 1973; Cooke, Mattick, \& Campbell, 1999; Orlandi, 1987).

Therefore, the goal of the present study was to assess and compare the relative importance of a comprehensive set of factors in two diffusion stages, viz. adoption and implementation. To this end, the results of four studies were compared. These studies focused on the diffusion of a stepwise protocol for smoking cessation support, the socalled Minimal Intervention Strategy for smoking cessation (MIS). This protocol includes the steps described in the 5 A's method (Fiore et al., 2000): ask, advise, assess, assist and arrange. Since the level of generalizability of study findings to other settings is generally difficult to judge (Foy, Eccles, \& Grimshaw, 2001; Glasgow, Lichtenstein, \& Marcus, 2003), two different types of health care setting were studied and compared, viz. cardiac wards and midwifery practices. The first is an example of hospital care, while the second is an example of primary care. We chose to compare the diffusion of a protocol in two different settings to obtain information on the generalizability of the diffusion process in different settings. 
The Integrated Change Model (I-Change model) served as our comprehensive theoretical framework (De Vries, Mesters, Van de Steeg, \& Honing, 2005; De Vries, Mudde, Leijs et al., 2003; De Vries, Mesters, Van 't Riet, Willems, \& Reubsaet, 2006). It was extended with factors derived from the contingency model of strategic decisionmaking (Koopman \& Pool,1991, 1994) (see Figure 1).

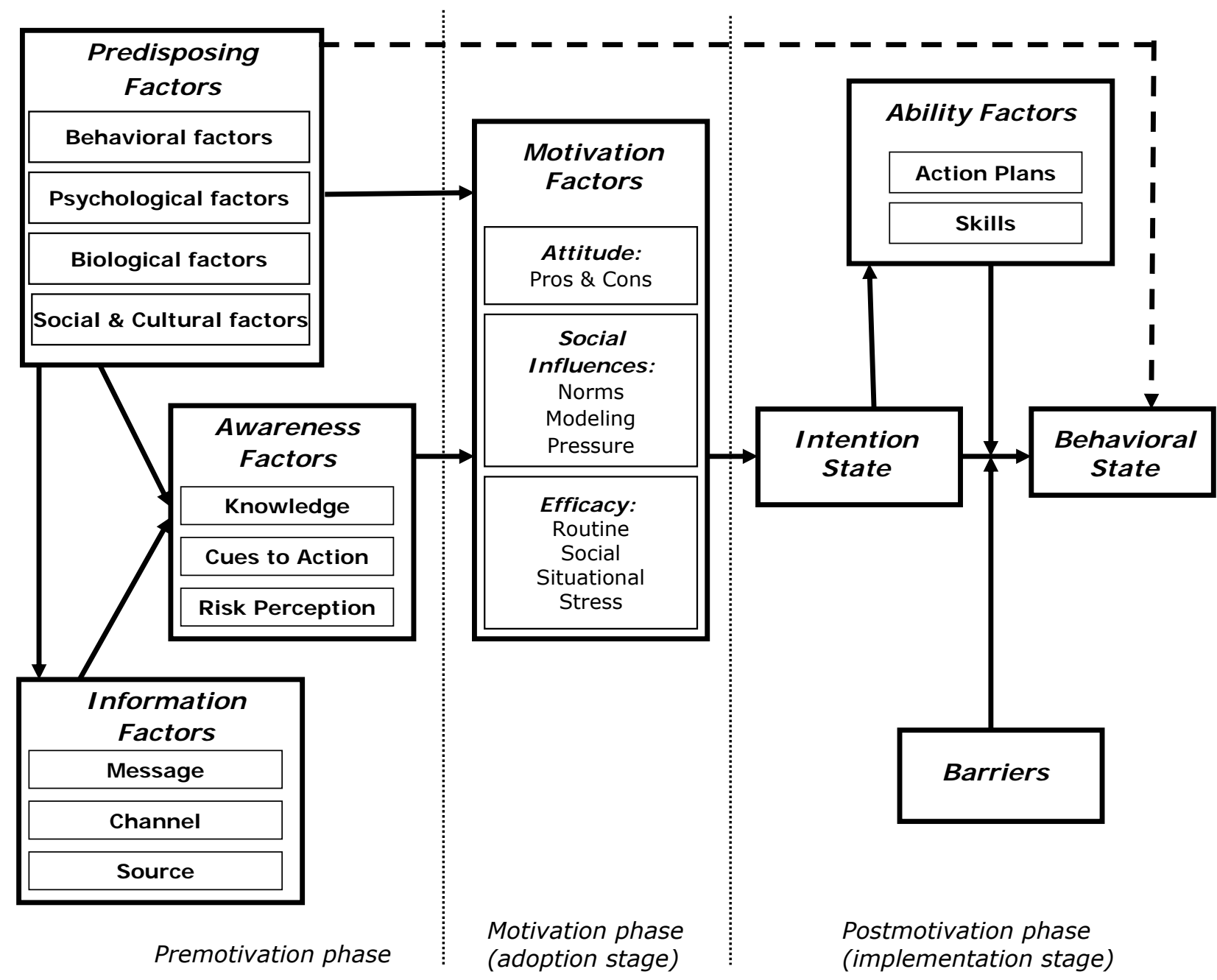

Figure 1. The Integrated Change model (De Vries et al., 2005, 2006; De Vries, Mudde, Leijs et al., 2003)

The I-Change Model builds on an earlier model referred to as the Attitude - Social Influence - Self Efficacy (ASE) model (De Vries \& Mudde, 1998) and integrates concepts of various social cognitive models (Ajzen, 1995; Bandura, 1986; Gollwitzer \& Brandstätter, 1997; Janz \& Becker, 1984; Prochaska \& DiClemente, 1983; Weinstein, 1988) and the diffusion theory (Rogers, 1995). This combination improves its ability to predict diffusion (Parcel et al., 1995). The model distinguishes three phases in behavioral change processes: pre-motivation, in which a person becomes aware of a problem and of the need for a new behavior or a change in an existing behavior (with knowledge, risk 
perceptions and cues to action assumed to be important), motivation, in which the intention to perform the new behavior is formed (with attitudes, social influences and self-efficacy assumed to be important) and post-motivation, in which intention is transformed into behavior (with action plans, skills and barriers assumed to be important). As the adoption of an intervention can be regarded as the positive intention to use it, the adoption stage was considered to be comparable to the motivation stage. Since the implementation of a protocol is the stage that targets the actual proper use of a protocol that one has decided on using before, we considered the implementation stage to be analogous to the post-motivation stage.

Apart from the social-cognitive factors that influence the pre-motivational, motivational and post-motivational phases, there are predisposing factors, including organizational factors, which are assumed in the I-Change model to influence behavior change, either directly or via pre-motivational or motivational factors. As the I-Change model does not comprehensively define all potentially relevant predisposing factors, we used the so-called contingency model of strategic decision-making (Koopman \& Pool, $1991,1994)$, taken from organizational psychology, to identify factors that are related to behavioral change in organizations. This model has been shown to be applicable in implementation research (Willemsen, Meijer, \& Jannink, 1999). The contingency model defines three major groups of characteristics influencing the decision-making process: the content of decision-making, its context and the dominant decision-making style within the organization. The context of decision-making consists of characteristics of the environment, of the organization and of the decision-maker. In studies on the diffusion of a specific intervention, the content of decision-making is the same for all healthcare professionals. In the present study, the content was the use of a protocol for providing smoking cessation support.

In sum, the present paper aims to identify which factors have the strongest associations with successful adoption and implementation of interventions among healthcare professionals. Due to lack of comparable research, the present paper is mainly explorative in nature. Nevertheless, some hypotheses were formulated, based on previous studies. First, based on the I-Change Model (De Vries et al., 2005; De Vries et al., 2003) we hypothesized that awareness factors and motivational factors would be significantly associated with both adoption and implementation in both settings. We expected the predisposing factors associated with diffusion to differ between stages and settings. Second, based on the findings by Cooke and colleagues (Cooke, 2000) we hypothesized that predisposing factors (including organizational factors) and motivational factors, would be about equally associated with adoption. Third, based on research showing that intention predicts actual behavior to a limited extent only and that the actual preparation of actions plays an important role (Webb \& Sheeran, 2006) we 
hypothesized that successful implementation would be at least equally closely associated with ability factors (post-motivation factors that play a role in the transformation of intentions into behavior) as with motivational factors. In line with this, we hypothesized that adoption would be much more closely associated with awareness and motivation factors than successful implementation. Lastly, previous research has found that the influence of attitude decreases and that of self-efficacy increases as individuals proceeded through Prochaska and DiClemente' s (Prochaska \& DiClemente, 1983) stages of behavioral change (De Vries \& Backbier, 1994; De Vries \& Mudde, 1998). Therefore, our fifth hypothesis was that this would also be true for proceeding though diffusion phases, and that the relative importance of self-efficacy would therefore increase and that of the attitude variables decrease along the diffusion phases.

\subsection{Methods}

\subsubsection{Study design and procedure}

Four cross-sectional datasets were analyzed and compared. One study (study A) was conducted among all cardiac inpatient wards in the Netherlands, represented by the heads of all such wards. Data about levels of adoption and determinants of adoption of the MIS were collected. Another study (study B) was conducted among cardiac nurses who worked on wards that had adopted the MIS. This study included a random sample of wards that adopted the MIS. In study B, we measured levels of use and collected information on potential determinants of successful implementation (operationalized as level of use) of the MIS. A third study (study C) was conducted among midwifery practices by approaching one representative of each midwifery practice in the Netherlands. As in study A, data about levels of adoption and determinants of adoption of the MIS were collected. A fourth study was conducted among midwives who worked in a random sample of practices that had adopted the MIS, and measured levels of use and potential determinants of successful implementation of the protocol.

Data on levels of adoption or use and on the potential determinants, including the variables of the I-Change Model (Figure 1), were collected by means of written questionnaires. The questionnaires on adoption (studies $\mathrm{A}$ and $\mathrm{C}$ ) were accompanied by $\mathrm{a}$ description of the MIS protocol for those who were unfamiliar with the protocol. In each study, ten $€ 25$ vouchers were raffled among the respondents. Postal reminders were sent to non-responders two or three weeks after the first mailing. Table 1 shows further details of the studies (e.g. sample sizes and response rates).

Based on their answers, respondents were classified as "adopters" or "nonadopters" in the adoption studies, and as "adherent users" or "non-adherent users" in the implementation studies (for more information see the outcome measures section 7.2.3). Next, the strengths of the associations between the potential determinants and adoption 
or adherence were assessed within each of the two settings. Finally, these associations were compared between studies.

Table 1. Study characteristics

\begin{tabular}{|c|c|c|c|c|}
\hline & Study A & Study B & Study C & Study D \\
\hline Setting & Cardiac wards & Cardiac wards & $\begin{array}{l}\text { Midwifery } \\
\text { practices }\end{array}$ & Midwifery practices \\
\hline Outcome & Adoption & $\begin{array}{l}\text { Successful } \\
\text { implementation } \\
\text { (Adherence) }\end{array}$ & Adoption & $\begin{array}{l}\text { Successful } \\
\text { implementation } \\
\text { (Adherence) }\end{array}$ \\
\hline Study population & $\begin{array}{l}\text { Cardiac ward } \\
\text { heads }\end{array}$ & $\begin{array}{l}\text { Cardiac nurses from } \\
\text { wards that had } \\
\text { adopted the MIS }\end{array}$ & $\begin{array}{l}\text { Midwifery practice } \\
\text { representatives }\end{array}$ & $\begin{array}{l}\text { Midwives from } \\
\text { practices that had } \\
\text { adopted the MIS }\end{array}$ \\
\hline $\begin{array}{l}\text { Timing of } \\
\text { measurement }\end{array}$ & $\begin{array}{l}4 \text { years after } \\
\text { nationwide } \\
\text { introduction of } \\
\text { the MIS }\end{array}$ & $\begin{array}{l}5 \text { years after } \\
\text { nationwide } \\
\text { introduction of the } \\
\text { MIS }\end{array}$ & $\begin{array}{l}2 \text { years after } \\
\text { nationwide } \\
\text { introduction of } \\
\text { the MIS }\end{array}$ & $\begin{array}{l}3 \text { years after } \\
\text { nationwide } \\
\text { introduction of the } \\
\text { MIS }\end{array}$ \\
\hline Eligible subjects & 121 & $\begin{array}{l}\text { Random sample of } 20 \\
\text { (from 39) wards, } \\
\text { employing } 553 \text { nurses }\end{array}$ & 446 & 497 \\
\hline Sample approached & 121 & 553 & 446 & $\begin{array}{l}250 \text { (random } \\
\text { sample) }\end{array}$ \\
\hline $\begin{array}{l}\text { Respondents } \\
\text { (\% of total sample) }\end{array}$ & $77(64 \%)$ & $210(38 \%)$ & $251(56 \%)$ & $137(55 \%)$ \\
\hline $\begin{array}{l}\text { Included in current } \\
\text { analysis }\end{array}$ & 76 & 206 & 251 & 103 \\
\hline Reasons for exclusion & $\begin{array}{l}90 \% \text { missing } \\
\text { data }\end{array}$ & $\begin{array}{l}\text { Task-oriented ward \& } \\
\text { not responsible for the } \\
\text { MIS }(3 x) \text {, missing data } \\
\text { on outcome variable } \\
(1 x)\end{array}$ & - & $\begin{array}{l}\text { Never started using } \\
\text { the MIS }(33 x) \text {, } \\
\text { Missing data on } \\
\text { outcome variable } \\
\text { (1x) }\end{array}$ \\
\hline
\end{tabular}

\subsubsection{Questionnaires}

The questionnaires used in the four studies consisted of comparable scales and questions, and were all based on existing questionnaires or relevant scales, as well as information from literature reviews and earlier work (Bakker, 2001; Bakker, De Vries, Mullen, \& Kok, 2005; Bolman, De Vries, \& Mesters, 2002; Bolman, De Vries, \& Van Breukelen, 2002; Cooke, 2000; Mesters \& Meertens, 1999; Mudde, Willemsen, Kremers, \& De Vries, 2000; Steckler, Goodman, McLeroy, Davis, \& Koch, 1992).

All questionnaires included questions on generic predisposing factors, such as age, innovativeness and organizational decision-making style characteristics (e.g. centralization, formalization) and on setting-specific predisposing factors such as availability of practice assistants in midwifery practices. They also included questions on awareness factors (i.e. knowledge, personal relevance and risk perception), on motivational factors (i.e. advantages, disadvantages, social norms, social pressure, modeling and self-efficacy) and on current intention and behavior. Additionally, the two implementation studies included questions on the post-motivational ability factors, i.e. 
skills and goal actions (situation-specific actions and intervention card use). Table 2 describes in more detail the variables that were measured.

The wording of the questions was the same in all studies for all variables except workload, knowledge and modeling. With respect to workload, the questionnaires of studies B, C and D asked: "How do you perceive your workload?" (-3=very quiet, $+3=$ very busy). In study $A$, the study about adoption on cardiac wards, the workload of the ward head was not necessarily related to the workload of the other ward employees, who are the ones to execute the MIS protocol. Therefore, we asked if the ward generally had sufficient staff ( $-2=$ highly insufficient, $2=$ highly sufficient). In study $C$, about adoption in midwifery practices, the knowledge scale was based on answers to four yes/no questions about knowledge levels: "Have you ever heard of the MIS?", "Do you know what the MIS is?" "Do you know how the MIS works?" and "Do you have a clear idea of the implications of using the MIS?" In the implementation studies B and D, the knowledge scale was based on two questions: "Do you know what the MIS is?" and "Are you acquainted with all steps of the MIS?" ( $0=$ not at all, $4=$ very well).

In study $\mathrm{A}$, only one question on knowledge about the MIS was asked: "Had you ever heard of the MIS before receiving the questionnaire?" (yes/no). Since this question was too superficial to be compared with the knowledge scales in the other studies, it was not included in the present comparative analysis.

With respect to modeling, the wording of the questions was comparable in all studies, viz. "In your perception, how many of your colleagues are using the MIS?", but in study $A$ there were 5 answering categories, ranging from $0=$ none to $4=$ all, whereas in studies $B, C$ and $D$ there were 11 answering options, ranging from 0 to $100 \%(0,10$, $\ldots 100 \%)$, representing the perceived proportion of colleagues who use the MIS.

\subsubsection{Outcome measures}

The outcome variable in studies A and C was adoption of the MIS. The distinction between adopters and non-adopters was based on three questions. First, health professionals were asked if they were already using the MIS (yes/no). If the answer was "no", they were asked whether they intended to use the MIS in the future and if so when (in the next month, in the next six months, in the next year, ever but not within a year, or never). Non-adopters were those respondents who were not using the MIS and were not seriously planning to start using it in the near future. Near future was defined as six months from the time of measurement. Adopters were those respondents who either had already started to use the MIS or were seriously planning to use it in the near future. 
Table 2. Description of the measured variables

\begin{tabular}{|c|c|c|c|}
\hline Variable & Description & $\mathrm{N}$ items & Scale \\
\hline \multicolumn{4}{|c|}{ Predisposing factors } \\
\hline Age & Number of years (continuous) & 1 & \\
\hline Smoking behavior & Current smoker? (yes/no) & 1 & \\
\hline $\begin{array}{l}\text { Years working in } \\
\text { current profession }\end{array}$ & Number of years (continuous) & 1 & \\
\hline $\begin{array}{l}\text { Level of } \\
\text { innovativeness }\end{array}$ & $\begin{array}{l}\text { General attitude toward changes in working practice } \\
(-2=\text { very negative } /+2=\text { very positive })\end{array}$ & 1 & \\
\hline Centralization & $\begin{array}{l}\text { Degree to which decisions about the working methods } \\
\text { are made centrally or at individual level }(0=\text { fully } \\
\text { individual/ } 4=\text { fully central) }\end{array}$ & 1 & \\
\hline Formalization & $\begin{array}{l}\text { Degree to which working methods are generally based } \\
\text { on set procedures or people's own solutions. ( } 0=\text { fully } \\
\text { own solutions, } 4=\text { fully set procedures) }\end{array}$ & 1 & \\
\hline Information use & $\begin{array}{l}\text { Amount of information generally used when making } \\
\text { decisions about introducing new working methods. } \\
\text { ( } 0=\text { no information, } 4=\text { all possible information) }\end{array}$ & 1 & \\
\hline $\begin{array}{l}\text { External or internal } \\
\text { information sources }\end{array}$ & $\begin{array}{l}\text { Number of external information sources versus } \\
\text { internal sources used in decision-making. ( } 0=\text { only } \\
\text { internal, } 4=\text { only external) }\end{array}$ & 1 & \\
\hline Workload & Perceived workload @ & 1 & \\
\hline Organization size & $\begin{array}{l}\text { Number of midwives/nurses in organization } \\
\text { (continuous) }\end{array}$ & 1 & \\
\hline Assistants (m) & Availability of practice assistants (yes/no) & 1 & \\
\hline Involvement (c) & $\begin{array}{l}\text { Involvement in decisions with respect to MIS (- } \\
2=\text { disagree },+2=\text { agree })\end{array}$ & 3 & $a=0.80$ \\
\hline $\begin{array}{l}\text { Smoking room for } \\
\text { nurses on ward (c) }\end{array}$ & $\begin{array}{l}\text { Presence of a room on the ward where nurses can } \\
\text { smoke (yes/no) }\end{array}$ & 1 & \\
\hline \multicolumn{4}{|c|}{ Awareness factors } \\
\hline Knowledge & $\begin{array}{l}\text { Level of knowledge about the existence of the MIS and } \\
\text { its content } @\end{array}$ & $1-4^{@}$ & \\
\hline Personal relevance & $\begin{array}{l}\text { Scale of the importance of smoking cessation support } \\
\text { in general, among the target group and in the specific } \\
\text { setting concerned ( }-2=\text { disagree, }+2=\text { agree) }\end{array}$ & 2 & $\begin{array}{l}r a^{\#}=0.92, r b=0.72 \\
r c=0.50, r d=0.54\end{array}$ \\
\hline Risk perception & $\begin{array}{l}\text { Rating of the health risks of smoking ( } 1=\text { no risk, } \\
10=\text { very high risk) }\end{array}$ & 1 & \\
\hline \multicolumn{4}{|l|}{ Motivation factors } \\
\hline Advantages & $\begin{array}{l}\text { Scale of beliefs about the intervention characteristics } \\
\text { as defined by Rogers and about outcome expectations } \\
(-2=\text { disagree, }+2=\text { agree })\end{array}$ & $\begin{array}{l}10-15 \\
\text { items }\end{array}$ & $\begin{array}{l}a a^{\# \#}=0.92, a b=0.88 \\
a c=0.90, a d=0.85\end{array}$ \\
\hline Disadvantages & $\begin{array}{l}\text { Scale of beliefs about the intervention characteristics } \\
\text { as defined by Rogers and about outcome expectations } \\
(-2=\text { disagree },+2=\text { agree) }\end{array}$ & $\begin{array}{c}10 \\
\text { items }\end{array}$ & $\begin{array}{l}a a^{\# \#}=0.88, a b=0.87 \\
a c=0.79, a d=0.84\end{array}$ \\
\hline Social norms & $\begin{array}{l}\text { Index of perceived norms of people in the immediate } \\
\text { social environment }\end{array}$ & 4 & \\
\hline Social pressure & $\begin{array}{l}\text { Index of perceived social support or pressure of people } \\
\text { in the immediate social environment }\end{array}$ & 4 & \\
\hline Modeling & Perceived proportion of others using the MIS $@$ & 1 & \\
\hline Self-efficacy & Perceived ability to consistently administer the MIS & 1 & \\
\hline \multicolumn{4}{|l|}{ Ability factors } \\
\hline Skills & $\begin{array}{l}\text { Scale of skills necessary to administer individual } \\
\text { elements of the MIS }\end{array}$ & $7 / 8^{*}$ & $a b=0.90, a d=0.82$ \\
\hline Goal actions & Scale of actions that facilitate consistent use of the MIS & & \\
\hline $\begin{array}{l}\text { Intervention card } \\
\text { use }\end{array}$ & $\begin{array}{l}\text { Frequency of use of intervention card (decision } \\
\text { aid/reminder) when administering the MIS }\end{array}$ & & \\
\hline
\end{tabular}

(m) only relevant or available for midwifery practices (c) only relevant or available for cardiac wards

$@$ For more information on questions and answering options, see the methods section *The skills scale had one less question in the study on the MIS for cardiac wards, as cardiac nurses are not supposed to, and hence do not need the skills to, give stop smoking advice, since cardiologists take care of that "Correlation coefficient per study ${ }^{\# \# C r o n b a c h ' s ~ a l p h a ~ p e r ~ s t u d y ~}$ 
Implementation was considered to be successful when the target behavior was reached. With respect to the implementation of the MIS, this implied full use of or adherence to the MIS protocol. Therefore, the outcome variable in studies B and D was adherence. Level of adherence was assessed by questions about compliance with the elements of the MIS. These elements ( 9 for cardiac nurses and 15 for midwives) had been identified by groups of experts in a previous phase of the studies. The number of elements differed, as the two protocols were not identical due to their adaptation to the specific settings and patient situations. Health professionals (nurses, midwives) were asked to indicate how often they applied the elements of the MIS ( $0=$ never, $4=$ always). A sum score was calculated as a total score for adherence. In accordance with the views of an expert panel on guideline implementation, which concluded that striving to use a guideline "all of the time" might be unrealistic to expect but "most of the time" should be the goal (Gross et al., 2001), a cut-off point was defined at three quarters of the maximum value. If the health professionals' score was above the cut-off point, they were categorized as "adherent users"; otherwise they were classified as "non-adherent users".

\subsubsection{Statistical analyses}

With respect to our study goal, which was to identify the factors that have the strongest association with successful adoption and implementation of the MIS protocol in the two settings, we first calculated Pearson phi correlation between all variables and the outcome measures (either adoption versus non-adoption or adherence versus nonadherence), using SPSS. Dichotomous outcome measures were used for all studies, to make the correlation coefficients comparable.

To test our hypotheses on the relative associations of factors with adoption or implementation, we estimated the explained variances of the groups of factors in the IChange model (i.e. predisposing factors, awareness factors, motivational factors and ability factors), by calculating Nagelkerke's $\mathrm{R}^{2}$ using logistic regression analyses. One regression analysis was performed for each group of factors, using the ENTER procedure to include all variables belonging to a specific factor in the analysis. To test our hypotheses with respect to the differences in relative importance of variables between adoption and implementation and between cardiac wards and midwifery practices, four one-to-one comparisons between correlations were conducted for each variable: adoption versus successful implementation in cardiac wards, adoption versus successful implementation in midwifery practices, adoption in cardiac wards versus adoption in midwifery practices, and successful implementation in cardiac wards versus successful implementation in midwifery practices. The significance levels of the differences between two correlations were computed. For this purpose, correlation coefficients were transformed into Fisher's z-scores. The difference between the z-scores was divided by 
the standard error and compared with the critical value for the z-score, which is 1.96 for 95\% confidence and 2.58 for $99 \%$ confidence (Blalock, 1972).

To check whether any differences in associations that we found might be due to differences in mean scores for a variable, mean scores on the variables for all respondents were calculated per study. For example, if the professionals, on average, perceived many disadvantages, it was more likely that disadvantages were associated with diffusion than when they perceived very few disadvantages. Differences between the means were tested with Student's t-test for differences in means between two samples. These data are only presented when they were significant and relevant to the interpretation of the study results. All analyses were performed with SPSS version 14.0. Differences were considered significant when $p<0.05$.

\subsection{Results}

\subsubsection{Characteristics of the sample}

The mean age of the respondents varied among the four studies between 36 and 38 years, and they had, on average, been working in their current profession for between 11 and 15 years. The proportion of smokers ranged between $8 \%$ (study C) and $14 \%$ (study D). Cardiac wards employed an average of about 30 nurses, and midwifery practices about 4 midwives. Respondents of all studies were predominantly female, with only study A including a substantial number of male participants (35\%). No association was found between gender and adoption $(r=0.054)$. At the time of the study, $63 \%$ of the responding cardiac wards had adopted the MIS, while 53\% of the responding midwifery practices had adopted the MIS, 54\% of the responding nurses had successfully implemented the MIS, and $64 \%$ of the responding midwives had successfully implemented the MIS.

\subsubsection{Associations between variables and diffusion stages}

Table 3 shows the phi correlations between independent variables and the diffusion stages (adoption and successful implementation) as outcome variables for cardiac wards and midwifery practices. The table also shows significant differences between the phi associations of each independent variable with the outcome measure in the different studies and it shows the estimated Nagelkerke's $R^{2}$ of the groups of factors that resulted from the regression analyses. The main results shown in this table are presented below. Differences between the means are presented insofar as they are significant and relevant to the interpretation of the results. 
Table 3. Phi correlation between independent variables and the outcome variable (adoption/adherence).

\begin{tabular}{|c|c|c|c|c|c|c|}
\hline & Variable & $\begin{array}{l}\text { Study a } \\
(n=76)\end{array}$ & $\begin{array}{l}\text { Study b } \\
(n=206)\end{array}$ & $\begin{array}{l}\text { Study c } \\
(n=251)\end{array}$ & $\begin{array}{l}\text { Study d } \\
(n=103)\end{array}$ & $\begin{array}{l}\text { Significant } \\
\text { differences }\end{array}$ \\
\hline \multirow{17}{*}{$\begin{array}{l}\text { Predisposing } \\
\text { factors }\end{array}$} & Nagelkerke's $R^{2}$ & 0.27 & 0.22 & 0.14 & 0.25 & \\
\hline & Characteristics of decision-maker & & & & & \\
\hline & Age & 0.07 & $0.19 * *$ & 0.10 & 0.17 & \\
\hline & Smoking behavior & -0.19 & -0.12 & 0.05 & 0.00 & \\
\hline & Years working in current profession & 0.07 & $0.17 *$ & 0.07 & 0.10 & \\
\hline & Level of innovativeness & 0.14 & 0.11 & 0.07 & $0.25 *$ & \\
\hline & Organizational characteristics (general) & & & & & \\
\hline & Centralization & -0.04 & -0.06 & 0.10 & 0.09 & \\
\hline & Formalization & $-0.28 *$ & 0.02 & $0.16 *$ & $0.22 *$ & $\begin{array}{l}b>a * c>a * * \\
d>b * *\end{array}$ \\
\hline & Amount of information used & 0.18 & $0.18^{*}$ & -0.09 & 0.12 & $a>c^{*}$ \\
\hline & $\begin{array}{l}\text { Proportion of external vs. internal info } \\
\text { sources }\end{array}$ & 0.00 & 0.08 & -0.08 & -0.01 & \\
\hline & Workload & 0.06 & 0.07 & -0.11 & 0.06 & \\
\hline & Organization size & $0.25 *$ & -0.02 & 0.06 & -0.07 & $a>b *$ \\
\hline & Organizational characteristics (specific) & & & & & \\
\hline & Assistants & $x$ & $x$ & $0.18^{* *}$ & -0.07 & $c>d^{*}$ \\
\hline & Involvement & $x$ & $0.27 * *$ & $\mathrm{x}$ & $\mathrm{x}$ & \\
\hline & Smoking room for nurses & $x$ & $-0.19 * *$ & $x$ & $x$ & \\
\hline \multirow{4}{*}{$\begin{array}{l}\text { Awareness } \\
\text { factors }\end{array}$} & Nagelkerke's $R^{2}$ & 0.32 & 0.16 & 0.31 & 0.18 & \\
\hline & Knowledge $^{\$}$ & $0.49 * *$ & $0.28 * *$ & $0.49 * *$ & $0.24 *$ & $c>d^{*}$ \\
\hline & Personal relevance & 0.13 & $0.23 * *$ & $0.43 * *$ & $0.33 * *$ & $c>a *$ \\
\hline & Risk perception & 0.04 & 0.13 & $0.19 * *$ & 0.18 & \\
\hline \multirow{7}{*}{$\begin{array}{l}\text { Motivational } \\
\text { factors }\end{array}$} & Nagelkerke's $R^{2}$ & 0.42 & 0.22 & 0.56 & 0.16 & \\
\hline & Advantages & $0.41^{* *}$ & $0.36 * *$ & $0.46 * *$ & $0.21 *$ & $c>d^{*}$ \\
\hline & Disadvantages & $-0.42 * *$ & $-0.31 * *$ & $-0.49 * *$ & $-0.23 *$ & $d>c^{*}$ \\
\hline & Social norms & $0.50 * *$ & $0.21 * *$ & $0.48^{* *}$ & $0.21^{*}$ & $a>b * c>d *$ \\
\hline & Social pressure & $0.30 * *$ & $0.18^{*}$ & $0.25 * *$ & $0.26 * *$ & \\
\hline & Modeling & $0.29 *$ & 0.05 & $0.43 * *$ & -0.02 & $\mathrm{c}>\mathrm{d} * *$ \\
\hline & Self-efficacy & $0.26 *$ & $0.23 * *$ & $0.22 * *$ & 0.16 & \\
\hline \multirow[t]{5}{*}{ Ability factors } & Nagelkerke's $R^{2}$ & & 0.35 & & 0.24 & \\
\hline & Skills & $x$ & $0.29 * *$ & $x$ & $0.33 * *$ & \\
\hline & Goal actions & & & & & \\
\hline & Situation-specific & $\mathrm{x}$ & $0.17 *$ & $x$ & $0.23 *$ & \\
\hline & Intervention card use & $x$ & $0.51 * *$ & $x$ & $0.28 * *$ & $b>d *$ \\
\hline
\end{tabular}

$* \mathrm{p}<0.05 * * \mathrm{p}<0.01$, Study $\mathrm{a}=$ Adoption MIS for cardiac nurses, Study $\mathrm{b}=$ Adherence MIS for cardiac nurses, Study $c=$ Adoption MIS for midwives, Study $d=$ =Adherence MIS for midwives ${ }^{*}$ Studies compared: a \& c, b \& d, a $\& b, c \& d .{ }^{\$}$ Knowledge was measured too superficially in study $A$ to compare with the knowledge scales in the other studies, so it was not included in the comparative analyses.

Table 3 furthermore shows that, in general, no significant phi associations were found between smoking behavior, level of centralization, proportion of external versus internal information sources used, and workload and adoption or adherence on the one hand and in cardiac wards and midwifery practices on the other. Furthermore, Table 3 shows that, in line with our first hypothesis, awareness factors and motivational factors 
were significantly associated with both adoption and implementation in both settings and that the predisposing factors that were associated with diffusion differed between stages and settings. Phi correlations showed that knowledge, perceived advantages, disadvantages, social norms and pressure were significantly associated with adoption and adherence in both settings. The remaining variables were significantly associated with either adoption or successful implementation and/or in only one of the two settings.

\section{Factors associated with adoption}

\section{Similarities between Settings}

As Table 3 shows, the regression analyses for both cardiac wards and midwifery practices revealed that the highest estimated proportions of variance in adoption were associated with motivational factors ( $42 \%$ and $56 \%$ ). They were followed (in order of the strength of associations) by awareness factors (32\% and 31\%) and the predisposing factors assessed in our studies (27\% and 14\%). These findings are not in line with our second hypothesis, which held that predisposing factors and motivation factors would be about equally associated with adoption. With regard to the phi correlation coefficients, the table shows that, in both settings, knowledge and all motivational factors were significantly associated with adoption, and the four variables with the strongest associations were knowledge, fewer perceived disadvantages, more perceived advantages and more positive social norms.

\section{Dissimilarities between Settings}

Table 3 also shows that not all factors had a similar weight in predicting adoption in the cardiac and midwifery settings. Phi associations showed that the amount of information generally used for decision-making was positively associated with adoption on cardiac wards $(r=0.18)$, but negatively associated with adoption in midwifery practices $(r=-$ 0.09). Although neither of these the associations were significant, the difference between the two correlations was significant. The mean amount of information generally used when making decisions about introducing new working methods on cardiac wards $(M=3.04, S D=0.64)$ was significantly higher than in midwifery practices $(M=2.75$, $\mathrm{SD}=0.74)$.

Organization size was only significantly associated with adoption on cardiac wards $(r=0.25)$ and not in midwifery practices $(r=0.06)$, although the difference between the phi correlations was not significant. Formalization was negatively associated with adoption on cardiac wards $(r=-0.28)$, but positively associated in midwifery practices $(r=0.16)$. The availability of practice assistants was significantly associated with adoption in midwifery practices $(r=0.18)$ but does not apply to cardiac wards. 
Personal relevance was significantly more strongly associated with adoption in midwifery practices $(r=0.43)$ than on cardiac wards $(r=0.13)$. In the adoption study, the mean personal relevance was significantly lower $(p<0.01)$ in midwifery practices $(M=1.09$, $\mathrm{SD}=0.64)$ than among cardiac ward heads $(M=1.38, \mathrm{SD}=1.14)$.

Risk perception was significantly associated with the adoption of the MIS in midwifery practices $(r=0.19)$, though not on cardiac wards $(r=0.04)$. The difference in correlations, however, was not significant. On average, cardiac ward heads perceived significantly greater health risks of smoking for their patients $(M=9.36, S D=0.80)$ than representatives of midwifery practices $(M=8.60, S D=1.20)$.

\section{Factors associated with successful implementation}

\section{Similarities between Settings}

Table 3 shows that in both cardiac wards and midwifery practices, the lowest estimated proportions of variance in adoption were associated with awareness factors ( $16 \%$ and $18 \%$ ) and motivational factors (22\% and $18 \%$ ). Nevertheless, knowledge, personal relevance, advantages, disadvantages, social norms and social pressure were significantly associated with implementation. In both settings, all ability factors assessed, i.e. skills, situation-specific goal actions and intervention card use, were also significantly associated with adherence.

\section{Dissimilarities between Settings}

As Table 3 shows, the regression analyses revealed that on cardiac wards, ability factors were associated with by far the highest proportion of variance in adherence (35\%), while in midwifery practices, the level of association was comparable to those of the other groups of factors (24\%). Still, both findings agreed with our third hypothesis, that ability factors would be at least equally strongly associated with implementation as motivation factors. In midwifery practices, it was the predisposing factors which were associated with the highest proportion of variance in adherence (25\%), though hardly any more than the ability factors. On cardiac wards, predisposing factors were associated with the second highest proportion of variance (22\%).

In terms of the phi coefficients, innovativeness was shown to be significantly associated with adherence in midwifery practices $(r=0.25)$ but not on cardiac wards $(r=0.11)$. However, differences in correlations were not significant. Formalization was significantly more associated with adherence in midwifery practices $(r=0.22)$ than on cardiac wards $(r=0.02)$. Involvement in decision-making was positively associated with adherence on cardiac wards $(r=0.27)$ and the presence of a room on the cardiac ward where nurses could smoke was negatively associated with adherence on such wards ( $r=-$ $0.19)$. These characteristics do not apply to midwifery practices. Self-efficacy was found 
to be significantly associated with adherence to the MIS by cardiac nurses $(r=0.23)$, but not by midwives $(r=0.16)$. However, the actual difference between the correlations was quite small and not significant. Furthermore, the general mean self-efficacy towards applying the MIS to all smoking patients was significantly lower $(p<0.01)$ among midwives $(-1.12, \mathrm{SD}=1.83)$ than among nurses $(-0.33, \mathrm{SD}=1.66)$.

Finally, Table 3 shows that the phi association between intervention card use and adherence was significantly stronger on cardiac wards $(r=0.51)$ than in midwifery practices $(r=0.28)$. The mean level of use of the intervention cards was significantly higher $(p<0.01)$ among cardiac nurses $(2.74, S D=1.66)$ than among midwives $(1.92$, $\mathrm{SD}=1.59)$.

\section{Differences between diffusion stages}

\section{In both settings}

As can be seen in Table 3, the phi associations of most motivational factors with adherence were generally weaker than their associations with adoption, in both cardiac wards and midwifery practices. The table also shows that - in line with our fourth hypothesis - regression analysis revealed that the total association of motivational factors was also much stronger for adoption than for implementation, and that this was also true for awareness factors. Associations between social norms and adherence were significantly weaker than associations with adoption, and unlike adoption, adherence was not significantly associated with modeling.

In line with our final hypothesis, the relative level of association between selfefficacy and implementation, compared to the level of association between attitude and implementation, was higher than the relative level of association between self-efficacy and adoption, compared to the level of association between attitude and adoption. However, we did not find a higher absolute association between perceived self-efficacy and implementation.

In one of two settings

With respect to predisposing factors, there were several differences between diffusion stages at either cardiac wards or midwifery practices. On cardiac wards, formalization was significantly negatively associated $(r=-0.28)$ with adoption, and organization size was significantly positively associated $(r=0.25)$ with adoption, while no relations with adherence were found. The differences between the correlations were significant.

Having a practice assistant was found to be significantly associated with adoption $(r=0.18)$, but not with adherence $(r=-0.07)$ in midwifery practices. Differences between correlations were significant, but midwives in the implementation study also significantly more often reported having a practice assistant than those in the adoption study $(72 \%$ vs. $50 \%)$. 
With respect to awareness factors, knowledge was significantly more strongly associated with adoption $(r=0.49)$ than with adherence $(r=0.24)$ in midwifery practices. As regards motivational factors, advantages ( $r=0.46$ vs. $r=0.21)$, disadvantages $(r=-0.49$ vs. $r=-0.23$ ) and modeling ( $r=0.43$ vs. $r=-0.02$ ) differed significantly between adoption and adherence in midwifery practices.

\subsection{Discussion and conclusion}

\subsubsection{Discussion}

The present study aimed to identify the variables that were relatively strongly associated with successful diffusion (i.e. adoption and implementation) of the MIS smoking cessation intervention among healthcare practitioners. As we wanted to know whether and to what extent associations differed between diffusion stages and between different healthcare settings, two diffusion stages (adoption and implementation) and two settings (cardiac wards and midwifery practices) were studied. Comparisons between stages and settings showed several similarities and differences in factors associated with diffusion of the MIS.

\section{General}

In line with our first hypothesis, predisposing factors, awareness factors and motivational factors were all significantly associated with both adoption and implementation in both settings. As we expected, the predisposing factors that were associated with diffusion differed between diffusion stages and between settings, but in neither of the settings were significant associations found between successful adoption/implementation of the MIS on the one hand and smoking behavior, level of centralization, proportion of external versus internal information sources used, and workload on the other hand. Our study also showed that knowledge and the motivational factors of perceived advantages, disadvantages, social norms and social pressure were significantly associated with successful adoption and implementation of the MIS in both settings, but all other variables assessed were significantly associated with only one of the two diffusion stages and/or only one of the two settings.

\section{Adoption}

With respect to adoption, several differences were found between settings. Formalization was negatively associated with adoption on cardiac wards, but positively associated with adoption in midwifery practices. The positive association found for midwifery practices contradicts Rogers' theory (Rogers, 1995), which states that formalization inhibits consideration of an innovation process (adoption). This might be due to the relatively small size of midwifery practices, compared to the organizations in the studies on which Rogers' theory was based. The association between adoption and the amount of 
information gathered before a decision is made was positive for cardiac wards and negative for midwifery practices, and the difference was significant. This might be due to the fact that cardiac wards generally value information more highly than midwifery practices, as is indicated by the larger amount of information generally gathered before decision-making. With respect to awareness factors, personal relevance was found to be strongly related to adoption of the MIS in midwifery practices, but we did not find such a relation for cardiac wards. This probably reflects whether smoking cessation treatment is regarded as part of the professional task, which is generally speaking true for cardiac wards, but less so among midwifery practices.

Contrary to our second hypothesis, adoption was much more strongly associated with motivational factors than with predisposing factors, including organizational characteristics. This finding is not in line with the findings by Cooke and colleagues (Cooke, 2000), which suggested that organizational factors are very important, even more important than practitioner factors, during the earlier diffusion stages. The discrepancy with our results might be explained by the fact that Cooke and colleagues did not study practitioners' motivational characteristics as extensively as we did.

In both settings, knowledge, fewer perceived disadvantages, more perceived advantages and more positive social norms were the factors most strongly associated with adoption. This means that they are important factors in enhancing adoption. It should be noted, however, that knowledge was measured by only one question in the study on adoption on cardiac wards.

\section{Implementation}

Formalization was significantly associated with implementation in midwifery practices, but not on cardiac wards. The finding with respect to implementation in midwifery practices is in line with Rogers' theory (Rogers, 1995), which states that formalization facilitates implementation of an intervention. The reason for not finding an association on the cardiac wards is not clear.

Some of the setting-specific organizational characteristics were significantly associated with successful implementation. For example, involvement in decision-making was strongly associated with implementation of the MIS among cardiac nurses (this was not assessed in any of the other studies). This finding agrees with that of Cooke and colleagues (Cooke, 2000), who found that participation in decision-making was positively related to all diffusion stages.

Our third hypothesis, which proposed that ability factors would be at least equally strong associated with implementation as motivational factors, was confirmed. However, associations differed greatly between cardiac wards and midwifery practices. On the cardiac wards, ability factors had by far the strongest associations with implementation, while in 
midwifery practices, motivational factors and predisposing factors were about equally associated with successful implementation as ability factors. The four factors that had the strongest associations with adherence in the implementation phase included, in both settings, the ability factors of skills and intervention card use.

As regards ability factors, intervention card use was more strongly associated with adherence among cardiac nurses than among midwives. This association may have been affected by the higher level of intervention card use among cardiac nurses, but given the fact that the proportion of adherers among midwives was higher than among cardiac nurses, it is not likely that this caused the difference. An explanation for the difference might be that nurses work in a more hectic environment with more potential distractions than midwives, which could increase the need for a system to guide nurses through the consultation about smoking. It could also be that midwives were better able to apply the MIS based on their experience alone, without using an intervention card, than cardiac nurses, because they generally consult more people per month.

\section{Differences between adoption and implementation}

Significant associations between predisposing factors and diffusion were hardly ever consistent across diffusion stages. Larger cardiac wards were more likely to adopt the MIS, but size was not associated with implementation. The same was true for the presence of assistants in midwifery practices.

In line with our fourth hypothesis, awareness and motivational factors were much more strongly associated with adoption than with implementation. With respect to awareness factors, this can be attributed to the large association between knowledge and adoption. With respect to the motivational factors, this is probably due to the fact that adoption is much closer to intention, while implementation refers to the more distant concept of behavior. Surprisingly, we found no association at all between modeling and successful implementation. It seems that when an organization has decided to start using a protocol, and it is clear to the health professionals what is expected from them, they focus mainly on their own behavior and how to succeed in that, instead of on what exactly the others do.

In line with our final hypothesis, the relative association between self-efficacy and implementation, compared to the association between attitude and implementation, was higher than the relative association between self-efficacy and adoption, compared to the association between attitude and adoption. This is in line with findings on the influence of attitude and self-efficacy when individuals proceed through stages of behavioral change (De Vries \& Backbier, 1994; De Vries, Mudde, Dijkstra, \& Willemsen, 1998), where a smaller influence of attitude and a larger influence of self-efficacy has been found in the 
later stages of change. However, we did not find a higher absolute association between perceived self-efficacy and implementation.

\subsubsection{Limitations}

Our study was subject to certain limitations. First, it suffered from the limitations of the four studies we compared. These studies yielded only cross-sectional data, precluding causal inferences. Secondly, response rates which varied between 38 and $64 \%$ might have caused selection bias and overrepresentation of adopters and implementers in the population we surveyed. However, a comparison of the adoption and implementation rates found in these studies with data from an electronic database at STIVORO (the organization that coordinates the implementation of the MIS) yielded no indications for this. The ratio between adopters and non-adopters or between adherent and inadherent users may have had some small influence on the correlation coefficients. Related to this, the fact that the adopter/non-adopter or adherent/non-adherent ratios were not equal in all studies may have caused minor differences between the studies in terms of correlations. This may have caused either some overrating or underrating of the differences. However, this effect is very small and could only have affected results in the case of borderline significances. A third limitation is that a few questions or answering scales relating to the same variables were not exactly the same in all studies; this point was elaborated above, in the section that describes the questionnaire. The difference may have affected correlations and therefore hampered comparability. Some factors could not be measured in all studies (such as ability factors, availability of practice assistants) and could therefore not be compared at all.

\subsubsection{Conclusion and recommendations}

Despite these limitations, the present study has yielded useful information on the relative importance of a comprehensive set of variables for two diffusion stages, viz. adoption and successful implementation, in two different settings. The results can help to select the variables on which a diffusion strategy should be targeted to achieve more successful intervention use (Bartholomew, Parcel, \& Kok, 1998).

In conclusion, our results suggest that strategies for diffusing interventions on smoking cessation in healthcare may need to distinguish between stages in the diffusion process. In the adoption stage, these strategies may need to focus on increasing knowledge and on motivational factors, especially increasing perceived advantages and social norms and decreasing perceived disadvantages. In the implementation stage, strategies are likely to be more effective when they focus on ability factors. Hence, they should outline how skills to perform the intervention can be improved (e.g. by providing training) and they should provide intervention cards or similar systems to serve as a 
decision aid and reminder, and stimulate their use. Nevertheless, motivational factors also need attention in this phase. These recommendations are probably useful irrespective of the specific healthcare setting, though as long as the personal relevance of administering smoking cessation treatment is low among the target group it seems to form an important barrier to the diffusion of a smoking cessation intervention. This barrier should also be given enough attention in the diffusion strategy.

Although predisposing factors were not the most important factors related to diffusion, the significant associations we found suggest that predisposing factors should be taken into account, where possible, to optimize diffusion strategies. Because each setting and diffusion stage seems to have its unique predisposing variables that are strongly associated with diffusion, it is desirable to study the target setting in detail while designing the diffusion strategy. For example, we found that low-formalized midwifery practices should receive special attention in the intervention diffusion process, both in the adoption phase and the implementation phase. Further studies are needed to find out if this is also true for other small organizations in healthcare, such as general practices. Extra attention to the influence of formalization is needed when diffusing interventions to the more highly formalized cardiac wards, but only in the adoption phase, because in that phase it was found to be a an impeding factor.

As our results show that each diffusion stage needs its own approach, additional research to explore the determinants of the final stage in the diffusion process, i.e. the maintenance or continuation stage, will be needed. Studies in other settings are needed to verify our findings. Future studies should ideally be planned prior to program implementation, so that the data can be collected longitudinally and can be used to identify predictors rather than associations with diffusion. Furthermore, a longitudinal study could include potentially important factors that are impossible to measure in a cross-sectional study, such as implementation intentions (Gollwitzer, 1999) and baseline satisfaction with current behavior. 



\section{CHAPTER 8}

\section{General Discussion}

Both optimists and pessimists contribute to our society. The optimist invents the airplane and the pessimist the parachute. 


\subsection{I ntroduction}

This thesis reports on the diffusion of a minimal intervention strategy for smoking cessation guidance (MIS protocol) in two healthcare settings: cardiac wards (hospital setting) and midwifery practices (primary care office setting). The MIS is a step-wise approach to help healthcare workers provide smoking cessation support to patients. It is tailored to the patients' motivational level, and consists of the following actions: assessing a smoker's profile, advising them to stop smoking, enhancing or reinforcing their motivation to quit, addressing barriers and high-risk situations, setting a date for quitting, providing educational materials and providing follow-up care.

Smoking cessation guidance by health professionals can effectively reduce smoking rates (Lumley, Oliver, Chamberlain, \& Oakley, 2004; Rigotti, Munafo, Murphy, \& Stead, 2003). The MIS for cardiac wards (C-MIS) has proved to increase smoking cessation rates among cardiac inpatients three months after hospital discharge (point prevalent abstinence $52 \%$ vs. $39 \%$ in the control group) (Bolman, De Vries, \& Van Breukelen, 2002a) and the MIS for midwifery practices (V-MIS) has proved to decrease smoking rates among women during pregnancy up until six weeks post-partum (point prevalent abstinence $21 \%$ vs. $12 \%$ in the control group) (De Vries, Bakker, DolanMullen, \& Van Breukelen, in press). Nevertheless, it is also known that a substantial part of the potential public health effects of interventions may be lost due to non-use or improper use as a result of faulty diffusion (Cranney, Warren, Barton, Gardner, \& Walley, 2001; Glasgow, Lichtenstein, \& Marcus, 2003; Haines \& Donald, 1998).

A program for national diffusion of the C-MIS was carried out by STIVORO, the Dutch expert centre on tobacco control, between 2000 and 2004 and a similar program for the V-MIS between 2002 and 2004. During this period, implementation activities were funded by the Netherlands Organization for Health Research and Development. The aims of the C-MIS diffusion program were that at the end of the period, the C-MIS would be used on $66 \%$ of the cardiac inpatient wards, and that $33 \%$ of all nurses on cardiac inpatient wards would use the C-MIS. The aim of the V-MIS diffusion program was that at the end of the period, the V-MIS would be used by $50 \%$ of the midwives.

The research reported on in this thesis had three aims. The first was to assess the level and quality of use of the C-MIS and V-MIS in the Netherlands, including identification of the steps of the protocol that turned out to be most difficult to apply in daily practice. In addition, we assessed the potential gains that could be achieved if the C-MIS and V-MIS were to be used adequately and consistently by all nurses and midwives in the Netherlands. The second aim was to identify and describe factors that are related to a successful diffusion of these health promotion protocols among healthcare practitioners. The third aim was to assess the applicability of a comprehensive model explaining behavior change, viz. the Integrated Change Model (De Vries, Mesters, 
Van de Steeg, \& Honing, 2005; De Vries, Mudde, Leijs et al., 2003; De Vries, Mesters, Van 't Riet, Willems, \& Reubsaet, 2006) in diffusion research. Since facilitating and impeding factors were expected to differ between the stages of diffusion, two distinct diffusion stages were studied: adoption and implementation.

This final chapter summarizes and discusses the main findings and integrates the studies that have been reported on in the previous chapters. Section 8.2 discusses the level of use of the C-MIS and V-MIS, while section 8.3 presents the potential national gains if the C-MIS and V-MIS were to be used adequately and consistently by all Dutch cardiac nurses and midwives. Section 8.4 discusses the determinants of diffusion of both interventions, and section 8.5 reports on the applicability of the Integrated Change Model in diffusion research. In section 8.6, methodological issues are discussed, while section 8.7 provides scientific and practical conclusions and recommendations.

\subsection{Levels of adoption and use of the MIS}

\subsubsection{Levels of adoption and use of the C-MIS}

During the nationwide diffusion program for the C-MIS that was carried out by STIVORO from July 2000 till December 2004, 66 of the 121 (55\%) cardiac inpatient wards in Dutch hospitals adopted the C-MIS. This means that these wards seriously intended to use the MIS, and that they had at least taken the first essential step to achieve this aim, i.e. attending the implementation training course offered by STIVORO. The proportion of wards that adopted the C-MIS over time, represented by the date at which the ward staff attended the C-MIS implementation training course, is indicated in Figure 1.

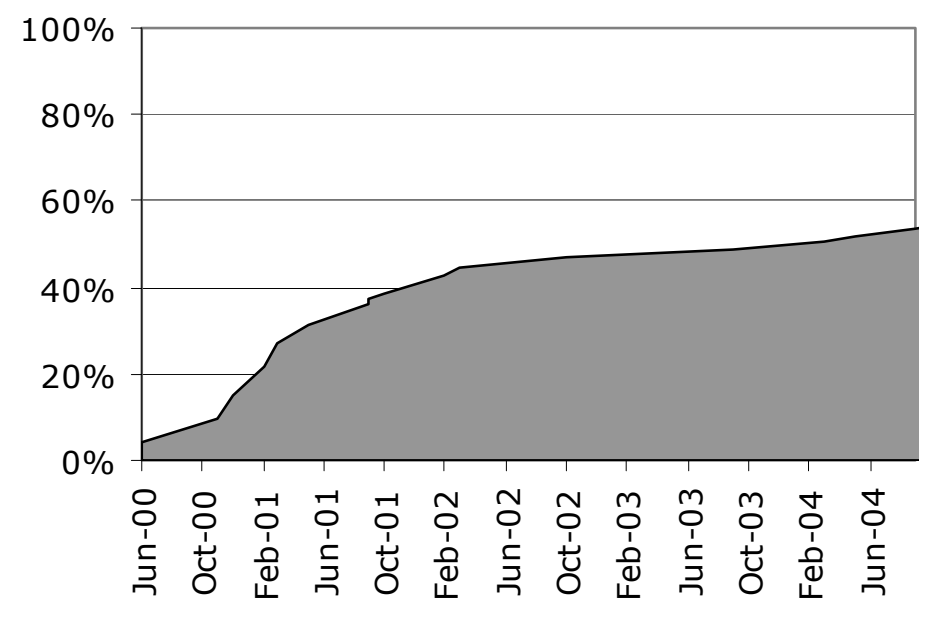

Figure 1. Proportion of wards adopting the C-MIS over time

Eventually, 55 of the 121 (45\%) wards had indeed implemented the C-MIS by December 2004 (Segaar \& Willemsen, 2004), which means that the nurses on these wards had started to use the C-MIS in daily practice. These wards employed $52 \%$ of all 
nurses that worked on cardiac inpatient wards. Chapter 3 showed that about $54 \%$ of these nurses managed to successfully implement the C-MIS in their daily practice after the implementation period, meaning that they consistently supported most of their smoking patients according to the steps of the C-MIS. Thus, $28 \%$ of all nurses on cardiac inpatient wards consistently and adequately used the C-MIS. The proportion of $54 \%$ consistent and adequate users of the C-MIS on wards that started to use the C-MIS is in line with the results of a previous Dutch pilot study among nurses on cardiac wards, which showed that $51 \%$ of the nurses on these wards used the C-MIS to support the majority or all of their patients (Bolman, De Vries, \& Mesters, 2002). This study, however, did not analyze the quality of the use of the C-MIS or the actual performance of all aspects of the C-MIS. The new data presented in chapter 3 of the present thesis show that aspects that were particularly under-applied were: providing smoking patients with the self-help guide about smoking cessation, discussing smoking cessation aids and providing follow-up care. The most important reasons for not providing patients with the self-help guide included that the guides were not always available on the wards and that nurses forgot to provide them. The most important reason for not discussing smoking cessation aids or not providing follow-up care was that some of the nurses felt they lacked the necessary skills. We concluded that additional training for these actions was needed.

\subsubsection{Levels of adoption and use of the V-MIS}

During the nationwide diffusion program for the V-MIS that was carried out by STIVORO from January 2002 until December 2004, 254 of the 450 midwifery practices (56\%) in the Netherlands adopted the V-MIS. This means that these practices seriously intended to use the MIS, and that they had at least taken the first essential step to achieve this aim, i.e. enrolling at least one of the midwives of the practice in the training course offered by STIVORO. The proportion of wards that adopted the V-MIS over time, represented by the date at which at least one midwife of the practice attended the training course on the V-MIS, is indicated in Figure 2. Eventually, 201 of the 450 (45\%) midwifery practices had indeed implemented the MIS by December 2004 (Segaar \& Willemsen, 2005), which means that the midwives in these practices had actually started to use the V-MIS in daily practice. These practices employed $59 \%$ of all primary care midwives. From chapter 5 it can be concluded that about $49 \%$ of the midwives in these practices successfully implemented the V-MIS in their daily practice after the implementation period, meaning that they consistently supported most of their smoking clients according to the steps of the V-MIS. Thus, $29 \%$ of all primary care midwives consistently and adequately applied the V-MIS. Not all actions required to use the V-MIS were easy to execute. The steps that turned out to be most difficult to perform 
consistently in the midwives' daily practice were discussing the barriers to smoking cessation, setting a quit date with motivated clients and providing follow-up care. These difficulties had already been identified in previous research (Bakker, Mullen, De Vries, \& Van Breukelen, 2003; Cooke, Mattick, \& Barclay, 1996) and the steps were still difficult to manage for the midwives, despite specially adapted implementation strategies that tried to overcome those difficulties. The most important reason that we found for not applying a particular step was a perceived lack of skills to perform this step among some of the midwives. We concluded that additional training for these actions was needed.

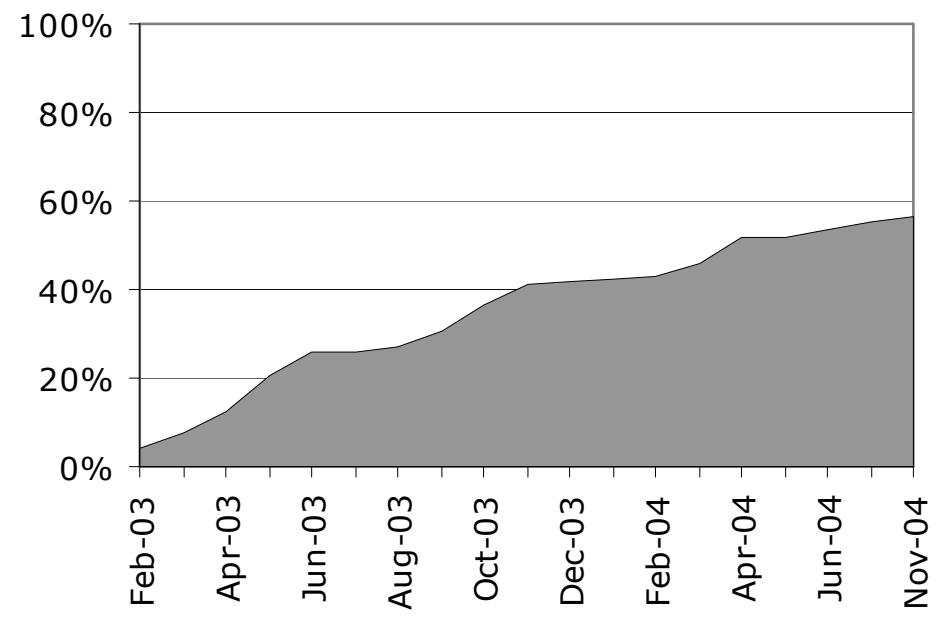

Figure 2. Proportion of practices adopting the V-MIS over time

8.2.3 Levels of use after implementation of the C-MIS and V-MIS compared to the H-MIS The levels of adoption and implementation of the C-MIS and V-MIS by health professionals after their diffusion period were much higher than the levels of adoption and implementation of the earlier MIS for general practitioners (H-MIS) after its two-year diffusion period. After two years, the proportion of general practitioners who had implemented the H-MIS was $21.5 \%$ (Pieterse, 1999), which is much lower than the figures of 52 and $59 \%$ for cardiac nurses (C-MIS) and midwives (V-MIS), respectively. The two-year implementation period of the H-MIS was much shorter than the four-year period used for the C-MIS, which may have caused some of the difference in implementation rates.

However, there may be several other reasons for the much higher implementation rates of the V-MIS and C-MIS compared to the H-MIS. First, unlike general practitioners, midwives and cardiac nurses treat specific high risk-groups, which increases the relevance of discussing the consequences of smoking. Furthermore, general practitioners generally work more independently than nurses and midwives; at the time of the study by Pieterse and colleagues that was performed in 1996, about $70 \%$ of the general practitioners were working in a one-person practice (NIVEL, 1996), whereas at the time 
of our 2005 study, $83 \%$ of all midwives were working in a group practice with at least 3 midwives (NIVEL, 2005) and cardiac nurses work on hospital wards together with many other nurses. Therefore, trying to convince general practitioners to adopt and implement the H-MIS required each and every one of them to be approached individually, which tends to take much more effort than approaching professionals in groups, such as cardiac wards or midwifery group practices. Pieterse et al. mentioned several important reasons for the low implementation degree: difficulties encountered, practical problems with the implementation of the MIS and the fact that most general practitioners did not attend the external training course (Pieterse, 1999). The completeness of use of the C-MIS and VMIS was comparable to that of the H-MIS. In all settings, the activities that were performed in most cases included discussing the motivation to quit and assessing the smokers' nicotine addiction, while the subsequent steps were performed less often. Whereas for the C-MIS and V-MIS it proved possible to identify the specific steps that were most difficult to perform consistently, the study on the H-MIS found that all steps other than discussing motivation and assessing addiction were equally likely to be omitted.

\subsection{Potential gains when using the C-MIS and V-MIS}

An important question is how many more smokers would successfully quit if all cardiac wards and all midwifery practices in the country were to implement the MIS fully and use it consistently and adequately. To answer this question, we first calculated, for cardiac wards as well as for midwifery practices, how many additional patients/clients would currently be expected to quit smoking as a result of the MIS, based on the most recent data on the level of use. We then calculated the number of additional patients/clients that could be expected to quit smoking as a result of the MIS if all nurses and midwives were to use the MIS consistently and adequately.

With respect to the C-MIS, the numbers of implementing cardiac wards and cardiac nurses were derived from the C-MIS implementation database (Segaar \& Willemsen, 2004). The proportion of adherent C-MIS users on the wards that implemented the C-MIS was derived from the study described in chapter 3, which found a figure of $54 \%$. The total number of patients admitted to Dutch cardiac inpatient wards per year is about 216,000 (Prismant, 2004) and the proportion of smokers among cardiac patients prior to hospital admission in the Netherlands is about $49 \%$ (Scholte Op Reimer et al., 2006). In our calculations, we assumed equal division of the patients among nurses. To calculate the potential number of additional quitters that could be expected if nurses consistently and adequately used the C-MIS, we assumed an increase in smoking cessation rates as a result of adherent use of the C-MIS of $13 \%$ three months after hospital discharge (Bolman, De Vries, \& Van Breukelen, 2002). 
With respect to the V-MIS, the numbers of implementing practices were derived from the V-MIS implementation database (Segaar \& Willemsen, 2005). The proportion of adherent users in the practices that implemented the V-MIS was derived from the study described in chapter 5 , which found a figure of $49 \%$. The total number of pregnant women in the Netherlands who visit a midwife per year is about 150,000 (CBS Statline, NIVEL, 2005), and the proportion of smokers among pregnant women is about $14 \%$ (Lanting, Van Wouwe, \& Crone, 2005). In our calculations, we assumed equal division of the clients among midwives. To calculate the potential number of additional quitters that could be expected if midwives consistently and adequately used the V-MIS, we assumed an increase in smoking cessation rates as a result of adherent use of the V-MIS of $9 \%$ at six weeks post-partum (De Vries et al., in press).

Figure 3 shows the estimated additional number of people that could be expected to quit per year as a result of the use of C-MIS, if it were to be fully implemented on all wards and if it were to be consistently and adequately used by all nurses on wards that implemented the C-MIS. Implementation on wards that had not implemented the C-MIS yet could increase the number of quitters by 6600 . However, it is very likely that, just as on the wards that have already implemented the MIS, non-adherent use will decrease the number of quitters ultimately achieved. Getting the current non-adherent users to use the C-MIS consistently and adequately could increase the number of quitters by 3300 .

Figure 4 shows the estimated additional number of people that could be expected to quit per year as a result of the use of the V-MIS if it were fully implemented in all practices and if it were to be consistently and adequately used by all midwives in practices that implemented the V-MIS. Implementation of the V-MIS in practices that had not implemented it yet could increase the number of quitters by 770 . However, it is very likely that, just as in practices that have already implemented the V-MIS, non-adherent use will decrease the number of quitters ultimately achieved. Getting the current nonadherent users to use the V-MIS consistently and adequately could increase the number of quitters by 570 .

These figures show that using the MIS could yield considerable gains in terms of extra quitters, if implementation rates and the quality of use could be increased. It can be estimated that - assuming that the whole target population of midwives and cardiac nurses would consistently use the MIS in their consultations with patients who smoke the number of patients who quit smoking as a result of the MIS could increase by about 3.5 times compared to the current estimated number of "MIS quitters" (total number of potential quitters as a result of the MIS if all cardiac nurses and midwives were to use the MIS consistently and adequately, divided by the number of current quitters as a result of the MIS achieved by the adherent users). The total number of quitters achieved per year 
thanks to the MIS would then be about 13,800 cardiac inpatients at three months postdischarge and 1,890 pregnant women at six weeks post-partum per year. These figures stress the importance of putting effort into both increasing implementation rates and increasing adherent use of existing effective interventions such as the MIS.

One might argue that there are serious limitations to what is realistically achievable in terms of implementation. However, according to Rogers, those who hold really traditional views and generally resist innovations comprise only $16 \%$ of all individuals (Rogers, 1995). It therefore seems realistic to assume that, with the right strategy, at least $84 \%$ of the target group could be convinced to use an intervention such as the MIS, which would produce a large gain. The information on determinants of adoption and successful implementation presented in the present thesis can be used to develop such a strategy.

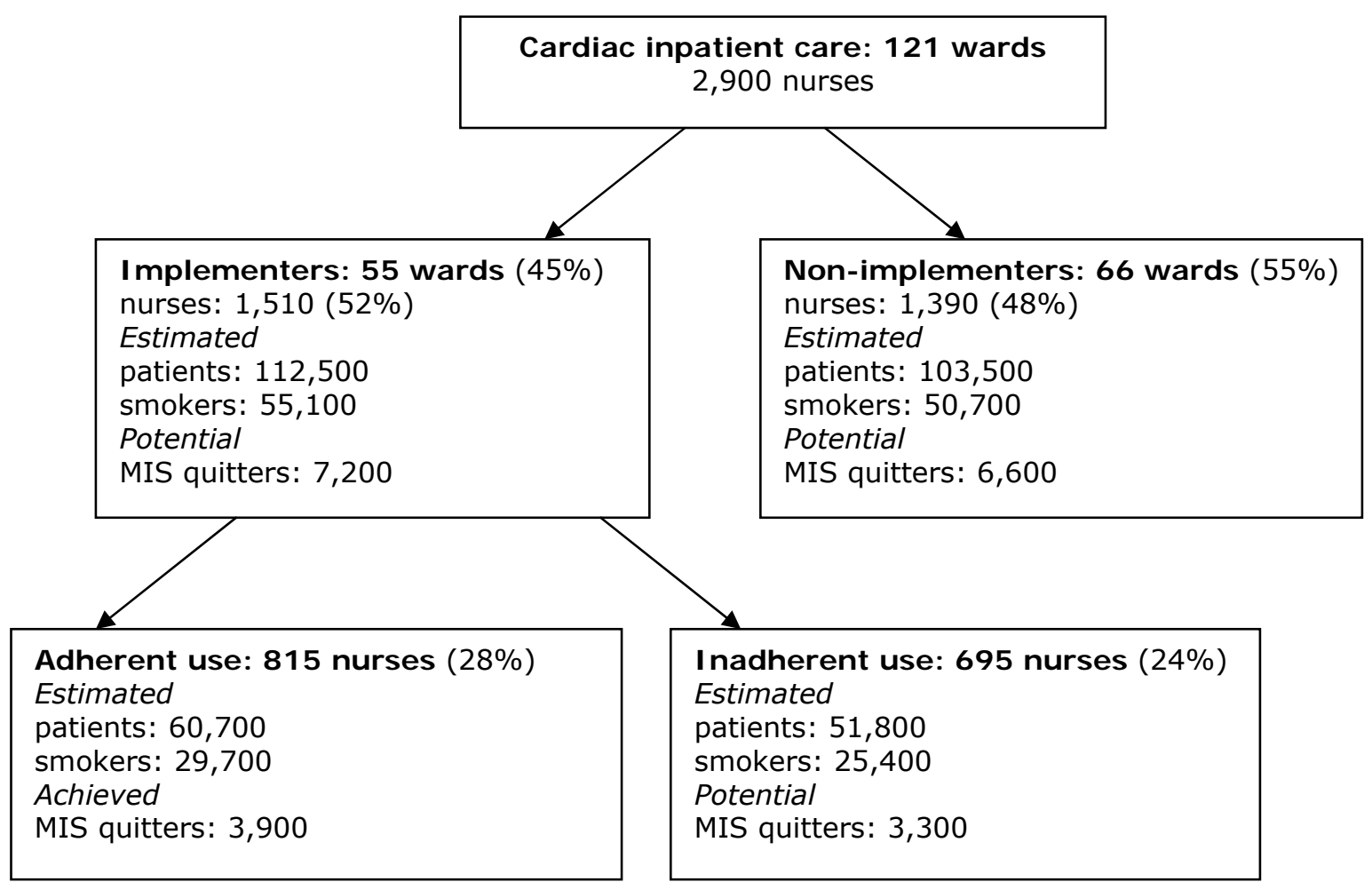

Figure 3. Use of the C-MIS and additional numbers of quitters achieved at 3 months after hospital discharge 


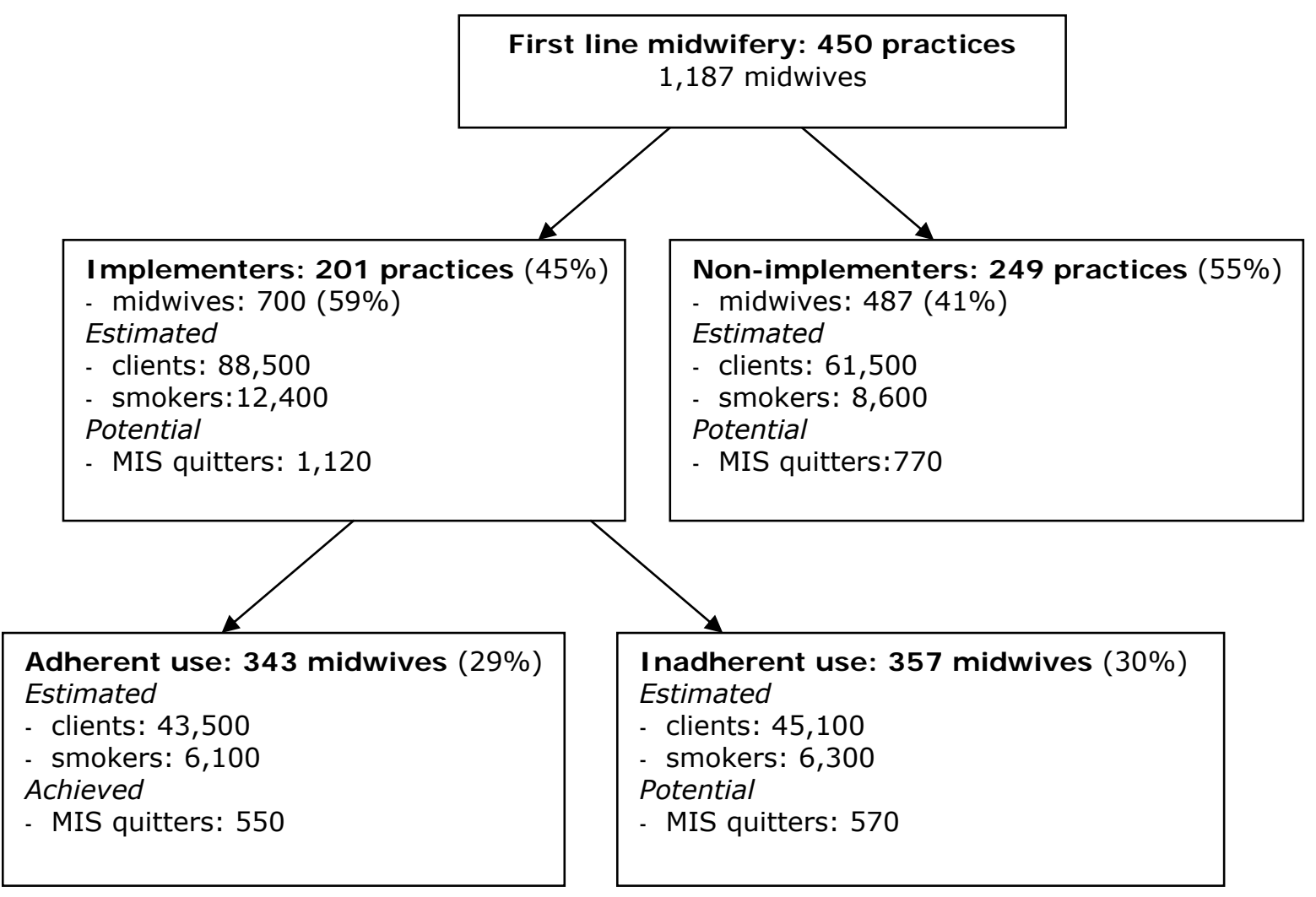

Figure 4. Use of the V-MIS and additional numbers of quitters achieved at 6 weeks postpartum

\subsection{Which factors facilitated or impeded the diffusion of the MIS protocols?}

The studies described in the present thesis aimed to identify the variables that were relatively important for successful diffusion of the MIS smoking cessation intervention among healthcare practitioners. The Integrated Change Model, which is a model to explain motivational and behavioral change, served as the comprehensive theoretical framework.

Contrary to most previous studies, the work reported on in the present thesis distinguished and compared between diffusion stages. Making a distinction between the stages is important, since the few studies that did this showed that the relative influence of many factors depends on the diffusion stage (Zaltman, Duncan, \& Holbek, 1973); (Cooke, Mattick, \& Campbell, 1999; Orlandi, 1987). We also chose to examine and compare the diffusion of a protocol in two different settings, to examine the generalizability of the diffusion process in different settings. The main facilitating and impeding factors that were found in the studies are reported in this section.

Chapter 7 showed that there were similarities and differences between the diffusion stages of adoption and implementation in terms of the relative importance of factors related to these stages. Similarities and differences also appeared to exist 
between cardiac wards and midwifery practices in terms of the importance of the different factors.

Our studies found several predisposing factors, including organizational factors, to be significantly associated with diffusion. This finding is in line with several theories, such as Rogers' diffusion theory (Rogers, 1995) and the contingency model of organizational decision-making by Koopman and Pool (Koopman \& Pool, 1991; Pool \& Koopman, 1992), as well as with several studies that found a significant influence of several organizational factors on diffusion, such as hospital size and centralization (Cooke, 2000; Parcel et al., 1995), hospital policy (Cooke, 2000), as well as capacity, professionalism and mission (Vaughn et al., 2002). However, significant associations between predisposing factors and diffusion were hardly ever consistent across diffusion stages or settings. We did not find any organizational factor that was consistently associated with diffusion in both the adoption and implementation stages or in both hospital wards and midwifery practices. This finding is in line with those of Dijkstra and colleagues (Dijkstra et al., 2006), whose meta-analysis found only limited evidence for the effects of organizational determinants on hospital care. Therefore, it is desirable to study a target setting in detail while designing the diffusion strategy, to identify specific facilitating and impeding factors for adoption and implementation in that particular setting.

Unlike predisposing factors, several awareness and motivation factors proved to be strongly associated with both adoption and implementation of the MIS in both settings. In line with previous findings by Mesters and colleagues (Mesters \& Meertens, 1999), we found awareness factors to be significantly associated with diffusion. Our studies also showed that the motivation factors of attitude, self-efficacy and social influences are important determinants of diffusion. This is in line with previous studies that found significant relations between the diffusion of various health promotion interventions on the one hand and attitudes towards the intervention (Fahrenwald \& Walker, 2003; Mesters \& Meertens, 1999; Haagen et al., 2005; Michie, Hendy, Smith, \& Adshead, 2004), perceived social influence (Paulussen, Kok, \& Schaalma, 1994; Wilhelm, 2002) and self-efficacy towards using the intervention (Wilhelm, 2002; Fahrenwald \& Walker, 2003; Haagen et al., 2005) on the other. However, these factors were much more strongly associated with adoption than with successful implementation.

In both cardiac wards and midwifery practices, adoption was most strongly related to knowledge of the MIS protocol and its steps, fewer perceived disadvantages, more perceived advantages and more positive social norms. This means that these are important factors in stimulating and enhancing adoption. Adoption was much more strongly associated with motivational factors than with predisposing factors, including organizational characteristics. This finding contradicts the findings by Cooke and colleagues, which suggested that organizational factors are very important, and even 
more important than practitioner factors, during the earlier diffusion stages. The discrepancy in results might be explained by the fact that Cooke and colleagues (Cooke, 2000) did not study the practitioners' motivational characteristics as extensively as we did.

Awareness factors and motivation factors were also relevant for implementation, but their relevance was much weaker than that for adoption. Additionally, implementation was strongly associated with the ability factors of skills and intervention card use, for both cardiac wards and midwifery practices.

The positive relation between use of a standardized protocol checklist (or intervention card) and protocol adherence had also been suggested by a study of Allewijnse and colleagues (Allewijnse, Mestemakers, Metsers, \& Van den Borne, 2003) in which physiotherapists indicated that working with a standardized protocol checklist for pelvic floor muscle exercise therapy had structured the content of their therapy sessions and made those sessions more efficient. In our studies the goal action of 'using the intervention card' had a direct influence on implementation behavior, additional to the other variables in the Integrated Change Model. This finding is in line with the idea that behavior is strongly affected by goal-directed actions, either conscious or unconscious, and the corresponding specific intentions to perform these actions, i.e. action plans (Lippke, Ziegelmann, \& Schwarzer, 2004; Sniehotta, Scholz, \& Schwarzer, 2005; Webb \& Sheeran, 2006).

Finally, longitudinal analyses of the predictive value of variables for implementation of the MIS within a one year period revealed that, in addition to knowledge and intention, dissatisfaction with current working methods significantly increased the chances of MIS implementation one year later (chapter 4).

\subsection{The I ntegrated Change Model in diffusion research}

Since adoption of an intervention can be regarded as the positive intention to use it, we considered the adoption stage to be comparable to the motivation stage in the Integrated Change Model. And since implementation of a protocol is the stage that targets actual proper use of a protocol that one has decided on using before, we considered the implementation stage to be analogous to the post-motivation stage in the I-Change Model.

In line with the I-Change Model, all groups of factors in the pre-motivation and motivation phases (predisposing, information, awareness and motivation factors) appeared to be significantly associated with adoption of the MIS, and all groups of factors in the pre-motivation, motivation and post-motivation stages were significantly associated with implementation of the MIS. Each of these groups of factors had individually also been found to be associated with diffusion in other studies (see also 
section 8.4). In the studies presented in this thesis, however, they were integrated in one model: the I-Change Model.

In our cross-sectional studies, the associative models consisting of significant factors from the I-Change Model were associated with 55 and $69 \%$ of the variance in adoption. This is higher than, for example, the $\mathrm{R}^{2}$ values $(0.41-0.50)$ found in a previous meta-analysis for the cross-sectional relation between intention and the concepts of attitude, subjective norm and perceived behavioral control from the theory of planned behavior (Armitage \& Conner, 2001). The studies included in this meta-analysis involved several types of behavior, ranging from health behavior to voting behavior, but no specific studies on diffusion were included. Our values are also higher than the $\mathrm{R}^{2}$ values found in previous studies on factors associated with the adoption of health promotion interventions. Paulussen and colleagues (Paulussen, Kok, Schaalma, \& Parcel, 1995) found $R^{2}$ values between 0.36 and 0.49 for the relation between curriculum-related beliefs, context and general dispositions on the one hand and the adoption of four AIDS curricula by Dutch secondary school teachers on the other. Cooke and colleagues (Cooke, 2000) found an $R^{2}$ of 0.29 for the relation between structural variables and the adoption of a smoking cessation program by antenatal clinics, and Mesters and colleagues (Mesters \& Meertens, 1999) found an $\mathrm{R}^{2}$ of 0.25 for the relation between awareness, concern and receptivity and the intention to adopt an educational protocol on pediatric asthma in family practices.

With respect to implementation, the associative models that consisted of significant factors from the I-Change Model were associated in our two studies with 52 and $56 \%$ of the variance in the cross-sectional studies. In our longitudinal study (chapter 4) the factors in the I-Change Model predicted $31 \%$ of the behavior after one year $(r=0.56)$. This is also slightly higher than the $R^{2}$ of 0.27 found in the meta-analysis by Armitage and Conner for the prediction of prospectively measured behavior using intention and concepts from the theory of planned behavior (Armitage \& Conner, 2001). Cooke and colleagues (Cooke, 2000) found an $\mathrm{R}^{2}$ of 0.24 for the relation between practitioner variables and the implementation of a smoking cessation program by antenatal clinics. Crone and colleagues (Crone et al., 2006) studied the completeness of use of an education program for the prevention of passive smoking among children in well-baby clinics, and found an $\mathrm{R}^{2}$ of 0.30 for the relation with user characteristics, characteristics of the dissemination strategy, involvement in the adoption decision and level of institutionalization in the production subsystem. Thus, our studies based on the I-Change Model provide a more extensive overview of factors that play an important role in adoption and implementation than previous studies.

Although the I-Change Model was tested cross-sectionally in most studies in this thesis, the findings indicated that the I-Change Model can be a useful framework for 
diffusion research when studying individuals within organizations. We found that all factors in the model were important in determining diffusion. Furthermore, the model explained a large proportion of the variance. Nevertheless, there is room for improvement and extension of the model for diffusion research. Firstly, a more extensive definition of ability factors would be valuable when using the model to predict successful implementation. The most recent version of the model (De Vries, Mesters, Van 't Riet, Willems, \& Reubsaet, 2006) defines the ability factors "actions plans" and "skills", with action plans refer to concrete intended actions.

A specific type of action plan that has received much attention recently is that of implementation intentions. Whereas action plans are specific plans to be carried out to allow the desired behavior to be performed - such as reading supportive educational materials - implementation intentions refer to one or a very limited set of action plans that are linked to an external cue, thus facilitating the eliciting of the desired behavior, for example planning to perform the behavior at a specific time, place and/or situation. Implementation intentions are considered to be a key element in the translation of intention into behavior (Gollwitzer, 1999; Webb \& Sheeran, 2006). According to Gollwitzer and colleagues (Gollwitzer, 1999), implementation intentions refer to anticipated situations and the accompanying behavioral plans that specify where, when and how goal-directed behaviors will be performed. We did not measure implementation intentions, though we did measure more generally defined goal actions, which can be defined as "sub-actions" facilitating the achievement of the intended final goal (behavior). Somewhat in line with studies on implementation intentions, we found a significant association between the implementation of the MIS and implementation of more general preparatory or facilitating goal actions (chapters 3, 5 and 6), such as talking with colleagues about the protocol or consulting a colleague in case of problems with applying the MIS. One goal action in particular - the level of use of an intervention card (decision aid) - was found to be significantly associated with successful implementation. These findings lead to the recommendation to include more general goal actions, as a separate concept next to specific action plans, in the ability factors in the IChange model for diffusion research.

A second issue regarding the I-Change Model concerns the concept of "cues to action" (part of the awareness factors in the model). There is a problem with the definition of the concept of cues to action in that there is a certain degree of freedom in interpreting it, a problem that also cropped up in its use within the Health Belief Model (Janz \& Becker, 1984; Sheeran \& Abraham, 1995). In our research, dissatisfaction was included as a cue to action, because dissatisfaction creates needs which in turn can lead to innovations (Rogers, 1995). Since we found that not only knowledge but also 
dissatisfaction was very important in predicting the long-term implementation of the MIS, we recommend focusing more explicit attention on this concept in the I-Change Model.

\subsection{Methodological issues}

Most methodological limitations of the studies have already been discussed in the previous chapters. This section provides an overview of the main weaknesses.

The levels of use were measured by means of self-report, which might have caused bias due to social desirable answers. Professionals could have overrated their level of use because they were expected to use the MIS. We tried to limit this by stressing the confidentiality of their response, by stating that there were no "wrong answers" and by stating that we were especially interested in identifying the steps and situations in which performing the protocol was more difficult or went wrong, so that we could adjust the protocol to their needs if necessary.

The levels of use that we found might also have been affected by selection bias, possibly resulting in overreporting by the health professionals of their adherence to the MIS protocol. However, in both adoption studies, the proportions of adopters among the respondents was about equal to the proportion of adopters among the total population as estimated from data in the electronic implementation databases maintained by STIVORO. And for cardiac nurses, there was no significant difference between the reported proportion of colleagues who used the C-MIS and the proportion of nurses who reported using the C-MIS themselves. The results regarding determinants were not likely to have been affected by the possible selection bias.

With respect to the variables that proved to be important within the various diffusion stages, our conclusions are limited by the fact that all but one of the studies were cross-sectional. Therefore, we were not able to verify if the variables associated with the diffusion stages did indeed predict these stages. Related to the cross-sectional nature of most studies is the limitation that not all variables could be adequately measured retrospectively. For example, although dissatisfaction was found to be highly associated with implementation of the MIS for midwives in the longitudinal study, retrospectively measured levels of satisfaction were not associated with adherence to the MIS in the cross-sectional studies. It is very likely that this is due to difficulties in measuring satisfaction retrospectively, although we cannot exclude the possibility that there is no relation between satisfaction with former working methods and the level of adherence to a new intervention.

Furthermore, some respondents in our study might have also participated in the effectiveness study of the MIS protocols (Bakker, 2001; Bolman, 2001). This might have affected the likelihood of adoption or successful implementation of the MIS. In our studies we did not take this possible effect into account. However, less than $5 \%$ of all 
cardiac wards ( 5 of 121 ) and all midwifery practices (22 of 446 ) performed the MIS as part of the effectiveness study. Their influence in the present studies is therefore probably low. Additionally, quite some time passed by between the end of the effectiveness study and the start of the national implementation of the MIS. For the CMIS this was three years and for the V-MIS this was five years. Another point of concern might be the fact that some respondents were asked to fill in both the adoption questionnaire and the implementation questionnaire. This was only true for some of the midwives, who were both a representative of their midwifery practice in the sample approached in the adoption study and an executing midwife in the sample approached in the implementation study. As the practices in our studies employed around four midwives on average, this will apply to about a quarter of the sample. For this group, it could be that answering the adoption questionnaire slightly affected their use of the MIS.

Finally, there were some imperfections with respect to the comparability of the studies. Not all variables could be measured in exactly the same way in all studies. This is due to insights improving as the project progressed. For example, knowledge was measured very superficially in the first study, on adoption on cardiac wards (chapter 2), compared to later studies. It is also due to differences in the situations we studied. For example, not all situations that occur on cardiac wards also apply to midwifery practices.

\subsection{Conclusions and recommendations}

\subsubsection{What did we learn scientifically?}

First, our results showed that the factors explaining adoption differed from those explaining implementation. This leads to the conclusion that each of these two diffusion stages needs its own approach. Given the difference between the adoption and implementation stages, it should be interesting to perform additional research to further examine the determinants of the final stage in the diffusion process, the maintenance stage. Furthermore, effectiveness trials will be needed to test the efficacy and effectiveness of different diffusion strategies for the different diffusion stages.

Second, our results showed that the motivation and ability factors had a similar influence on diffusion in both cardiac wards and midwifery practices. However, the predisposing factors that facilitated or impeded diffusion differed greatly between settings. Therefore, settings should be studied prior to the development of a final diffusion strategy, to identify which setting-specific predisposing factors are important. In a similar vein, more diffusion studies in other healthcare settings are needed to detect other potentially relevant characteristics of settings. These studies could verify the consistency across settings with respect to the importance of motivational factors and ability factors in diffusion, and also identify important setting-specific predisposing factors. It is likely that many of the factors that we identified also apply to the diffusion 
of health promotion interventions other than those for smoking cessation support. To verify the generalizability of our results to other interventions, more diffusion studies of this nature should be conducted with other interventions.

Third, our studies indicate that the I-Change Model can be a useful framework for diffusion research, when studying individuals within organizations, and that all factors identified in this model, i.e. predisposing, information, awareness, motivation and ability factors, contribute to the final behavior. However, because the model was tested crosssectionally in most of our studies, it will be useful to perform more longitudinal studies to verify the order and patterns assumed in the model. Additionally, it can be useful to perform some experimental research to gain more information on the specific effects of potentially important ability factors, such as action planning. As till now they only have been studied to a very limited extent. Furthermore, our studies indicate that when the IChange Model is used in diffusion research, it could be improved by including goal actions as a separate ability factor in addition to action plans and by giving explicit attention to the concept of satisfaction.

Additionally, with respect to future research we would recommend that studies on diffusion are planned prior to program implementation, so that the data can be collected longitudinally and therefore truly identify predictors of diffusion rather than associations with diffusion.

\subsubsection{What did we learn for practice?}

As was demonstrated in section 8.3, the potential gain in cessation rates that can be achieved by increasing the successful diffusion of the existing MIS intervention is very high. Therefore it is advisable to put much effort into facilitating the diffusion and stimulating the adoption and implementation of these existing interventions, rather than developing new interventions for the same target group. Additionally, we recommend the development of modifications to the MIS protocol that would make it easier for health professionals to consistently apply all intervention elements and to improve follow-up care.

Unfortunately, the findings in this thesis make it clear that there is no magic bullet that can achieve successful diffusion of the MIS in all situations. The finding that a large number of widely varying factors play a significant role in adoption and implementation shows that multifaceted diffusion strategies (strategies consisting of multiple elements) are necessary to increase adoption and implementation rates. As these strategies can target more impeding factors simultaneously, they are generally more effective than strategies consisting of only one element, provided that they target the right barriers (Hulscher, Wensing, Van der Weijden, \& Grol, 2003). 
Given the differences that we found between the two diffusion stages and between the two settings, it is impossible to recommend one multifaceted approach to promote adoption and implementation of interventions for smoking cessation support in healthcare. Therefore, we recommend developing and applying setting- and stagetailored diffusion strategies that consist of multiple elements. This means that one should be flexible when developing and executing diffusion strategies and study the target setting prior to designing stage-specific diffusion strategies. The last could be done for example by interviewing or observing people that operate in the specific setting and by searching for information on the setting in the literature.

In spite of the differences between diffusion stages and between settings, there are some general recommendations we can make that are likely to be valid for both the adoption and implementation stages and for a variety of healthcare settings. Knowledge, motivation and skills to perform the MIS should be increased. This could be achieved by interactive educational meetings, as they have proved to be effective in increasing the use of interventions by professionals (Davis et al., 1999; Thomson O'Brien et al., 2001). Although large didactic educational sessions are a much cheaper way to reach a large group of people, they will hardly ever increase intervention use (Grimshaw et al., 2004). Therefore, they should only be used in the adoption stage, where increasing knowledge is an important goal. In the implementation stage or its preparations, there is a need for interactive educational meetings. Our studies showed that professionals who used the MIS still experienced a lot of difficulties in consistently and adequately applying the MIS, and that lacking the necessary skills to perform some of the steps of the MIS was a major barrier. Therefore, professionals should be offered continuing education (so called booster sessions) once in a while, in which special attention is given to the most difficult elements, such as discussing smoking cessation aids with patients and providing followup care (for cardiac nurses) and discussing barriers, setting a quit date and providing follow-up care (for midwives).

In the implementation stage, it is also very important to provide professionals with intervention cards or other comparable systems that serve as a protocol application aid and reminder, and stimulate them to use the intervention. As computers are increasingly being used in everyday healthcare, a computerized intervention card might be a good option. Computerized clinical decision support systems have proved their potential effectiveness in improving care performance (Garg et al., 2005).

Since social influence was found to have an important effect on the diffusion of the MIS, it is important to use implementation strategies that focus on creating stimulating social influences, such as audit and feedback and the involvement of opinion leaders. Audit and feedback seem to be suitable to increase adherent intervention use. It is a feasible method to provide care professionals with information on their own 
behavior, in comparison with that of their peers. This information has been found to motivate healthcare professionals to improve their behavior (Jamtvedt, Young, Kristoffersen, O'Brien, \& Oxman, 2006). As non-adopters and non-adherent implementers are generally less convinced of the effect of their efforts on the smoking behavior of their patients, it is advisable to include information on the behavior of the target population in this feedback information and so make the effect of the intervention on the smoking behavior of their patients more visible to the practitioners. However, this type of information should relate to smoking cessation rates among clients of the entire professional group that uses the MIS, rather than to clients of an individual professional only. The number of clients per professional is in fact so small that for each individual practitioner, the proportion of smokers induced by MIS to quit smoking would depend too much on chance. Furthermore, local opinion leaders should be approached to involve them in strategic action to create more positive norms towards the use of the intervention (Thomson O'Brien et al., 2000), which in turn should increase the adoption and implementation of an intervention.

Lastly, it is very important to monitor and support those organizations and professionals that have already successfully implemented the intervention, to prevent them from inadequately using the intervention or giving it up because they lose their motivation. They should remain committed to the intervention, for example by being sent a newsletter once in a while. Their stories can be used as a "good practice" example for others. 


\section{References}

Ajzen, I. (1991). The theory of planned behavior. Organizational behavior and human decision processes, 50, 179-211.

Ajzen, I. (1995). From intentions to actions: A theory of planned behavior. In J. Kuhl \& S. J. Beekman (Eds.), Action-control: From cognition to behavior (pp. 11-39). Heidelberg: Springer.

Ajzen, I. (2001). Nature and operation of attitudes. Annual Review of Psychology, 52, 27-58.

Alewijnse, D., Mesters, I., Metsemakers, J., Van den Borne, B. (2003) Predictors of longterm adherence to pelvic floor muscle exercise therapy among women with urinary incontinence. Health Education Research, 18, 511-523.

Armitage, C. J., \& Conner, M. (2001). Efficacy of the Theory of Planned Behaviour: a meta-analytic review. British Journal of Social Psychology, 40(Pt 4), 471-499.

Bakker, M. J. (2001). Pregnancy, a window of opportunity to quit smoking! PhD Thesis: University of Maastricht, Maastricht.

Bakker, M. J., De Vries, H., Mullen, P. D., \& Kok, G. (2005). Predictors of perceiving smoking cessation counselling as a midwife's role: a survey of Dutch midwives. European Journal of Public Health, 15, 39-42.

Bakker, M. J., Mullen, P. D., De Vries, H., \& Van Breukelen, G. (2003). Feasibility of implementation of a Dutch smoking cessation and relapse prevention protocol for pregnant women. Patient Education and Counseling, 49, 35-43.

Bandura, A. (1986). Social foundations of thought and action: A social cognitive theory. Englewood Cliff: Prentice Hall.

Bartholomew, L. K., Parcel, G. S., \& Kok, G. (1998). Intervention mapping: a process for developing theory- and evidence-based health education programs. Health Education \& Behavior, 25, 545-563.

Berkel, T. F. M. (2000). Smoking cessation as secondary prevention for patients with coronary artery disease. PhD Thesis: Erasmus Universiteit Rotterdam, Rotterdam.

Bero, L. A., Grilli, R., Grimshaw, J. M., Harvey, E., Oxman, A. D., \& Thomson, M. A. (1998). Closing the gap between research and practice: an overview of systematic reviews of interventions to promote the implementation of research findings. The Cochrane Effective Practice and Organization of Care Review Group. British Medical Journal, 317, 465-468.

Blalock, H. (1972). Social Statistics. New York: McGraw-Hill.

Bogers, R. P., Brug, J., van Assema, P., \& Dagnelie, P. C. (2004). Explaining fruit and vegetable consumption: the theory of planned behaviour and misconception of personal intake levels. Appetite, 42, 157-166. 
Bolman C., De Vries H., \& Mesters I. (2002). Factors determining cardiac nurses' intentions to continue using a smoking cessation protocol. Heart and Lung, 31, 15-24.

Bolman, C. (2001). Smoking cessation among patients hospitalized with cardiac disease: Evaluation of a minimal-contact intervention. PhD Thesis: University of Maastricht, Maastricht.

Bolman, C., De Vries, H., \& Van Breukelen, G. (2002a). Evaluation of a nurse-managed minimal-contact smoking cessation intervention for cardiac inpatients. Health Education Research, 17, 99-116.

Bolman, C., De Vries, H., \& Van Breukelen, G. (2002b). A minimal-contact intervention for cardiac inpatients: long-term effects on smoking cessation. Preventive Medicine, 35, 181-192.

Borrelli, B., Hecht, J. P., Papandonatos, G. D., Emmons, K. M., Tatewosian, L. R., \& Abrams, D. B. (2001). Smoking-cessation counseling in the home. Attitudes, beliefs, and behaviors of home healthcare nurses. American Journal of Preventive Medicine, 21, 272-277.

Bostrom, J., \& Wise, L. (1994). Closing the gap between research and practice. Journal of Nursing Administration, 24, 22-27.

Braspenning, J. C. C., Schellevis, F. G., \& Grol, R. (2004). Tweede nationale studie naar ziekten en verrichtingen in de huisartspraktijk. Kwaliteit huisartsenzorg belicht. Utrecht/Nijmegen: Nivel/WOK.

British Medical Association (2004). Smoking and Reproductive Life- The Impact of Smoking on Sexual, Reporductive and Child Health. Edinburgh: BMA-Board of Science and Education and Tobacco Control Resource Centre.

Burns, D. M. (2003). Epidemiology of Smoking-Induced Cardiovascular Disease. Progress in Cardiovascular Diseases, 46, 11-29.

Castle, N. G. (2001). Innovation in nursing homes: which facilities are the early adopters? Gerontologist, 41, 161-172.

CBO Kwaliteitsinstituut voor de Gezondheidszorg (2004). Richtlijn Behandeling van tabaksverslaving. Alphen aan den Rijn: Van Zuiden Communications b.v.

Cooke M., Mattick R.P., \& Campbell E. (2000). A description of the adoption of the 'Fresh start' smoking cessation program by antenatal clinic managers. Australian Journal of Advanced Nursing, 18, 13-21.

Cooke, M. (2000). The dissemination of a smoking cessation program: predictors of program awareness, adoption and maintenance. Health Promotion International, 15, 113-123.

Cooke, M., Mattick, R. P., \& Barclay, L. (1996). Predictors of brief smoking intervention in a midwifery setting. Addiction, 91, 1715-1725. 
Cooke, M., Mattick, R. P., \& Campbell, E. (1999). The dissemination of a smoking cessation program to 23 antenatal clinics: the predictors of initial program adoption by managers. Australian and New Zealand Journal of Public Health, 23, 99-103.

Cranney, M., Warren, E., Barton, S., Gardner, K., \& Walley, T. (2001). Why do GPs not implement evidence-based guidelines? A descriptive study. Family Practice, 18, 359-363.

Critchley, J., \& Capewell, S. (2004). Smoking cessation for the secondary prevention of coronary heart disease. Cochrane Database of Systematic Reviews, CD003041.

Crone, M. R., Verlaan, M., Willemsen, M. C., Van Soelen, P., Reijneveld, S. A., Sing, R. A., et al. (2006). Sustainability of the prevention of passive infant smoking within well-baby clinics. Health Education and Behavior, 33, 178-196.

Damanpour, F. (1991). Organizational innovation: a meta-analysis of effects of determinants and moderators. Academy of Management Journal, 34, 555-590.

Davis, D. A., \& Taylor-Vaisey, A. (1997). Translating guidelines into practice. A systematic review of theoretic concepts, practical experience and research evidence in the adoption of clinical practice guidelines. Canadian Medical Association Journal, 157, 408-416.

Davis, D., O'Brien, M. A., Freemantle, N., Wolf, F. M., Mazmanian, P., \& Taylor-Vaisey, A. (1999). Impact of formal continuing medical education: do conferences, workshops, rounds, and other traditional continuing education activities change physician behavior or health care outcomes? Journal of the American Medical Association, 282, 867-874.

De Vries H., Backbier E., Dijkstra M., Van Breukelen G., Parcel G., \& Kok G. (1994). A Dutch social influence smoking prevention approach for vocational school students. Health Education Research, 9, 365-374.

De Vries H., Bakker M., Dolan-Mullen P., \& Van Breukelen G.(in press). The effects of smoking cessation counseling by midwives on Dutch pregnant women and their partners. Patient Education and Counseling.

De Vries H., Mesters I., Van de Steeg H., \& Honing C. (2005). The general public's information needs and perceptions regarding hereditary cancer: an application of the Integrated Change Model. Patient Education and Counseling, 56, 154-165.

De Vries, H. (1998). Planning and evaluating health promotion. London: Stanley Thornes.

De Vries, H., \& Backbier, E. (1994). Self-efficacy as an important determinant of quitting among pregnant women who smoke: the phi-pattern. Preventive Medicine, 23, 167-174. 
De Vries, H., \& Kok, G. (1996). Gezondheidsbevordering: een toepassing van het ABC planningsmodel [Health promotion: an application of the $A B C$ planning model]. Gedrag en gezondheid, 24, 342-352.

De Vries, H., \& Mudde, A. (1998). Predicting stage transitions for smoking cessation applying the attitude-social influence-efficacy model. Psychology and Health, 13, 369-385.

De Vries, H., Backbier, E., Kok, G., \& Dijkstra, M. (1995). The impact of social influences in the context of attitude, self-efficacy, intention and previous behaviour as predictors of smoking onset. Journal of Applied Social Psychology, 25, 237-257.

De Vries, H., Dijkstra, M., \& Kuhlman, P. (1988). Self-efficacy: the third factor besides attitude and subjective norm as a predictor of behavioral intentions. Health Education Research, 3, 273-282.

De Vries, H., Mesters, I., Van 't Riet, J., Willems, K., \& Reubsaet, A. (2006). Motives of Belgian adolescents' for using sunscreen: the role of action plans. Cancer, Epidemiology and Biomarkers, 15, 1360-6.

De Vries, H., Mudde, A. N., Dijkstra, A., \& Willemsen, M. C. (1998). Differential beliefs, perceived social influences, and self-efficacy expectations among smokers in various motivational phases. Preventive Medicine, 27, 681-689.

De Vries, H., Mudde, A., Kremers, S., Wetzels, J., Uiters, E., Ariza, C., et al. (2003). The European Smoking Prevention Framework Approach (ESFA): short-term effects. Health Education Research, 18, 649-663; discussion 664-677.

De Vries, H., Mudde, A., Leijs, I., Charlton, A., Vartiainen, E., Buijs, G., et al. (2003). The European Smoking Prevention Framework Approach (EFSA): an example of integral prevention. Health Education Research, 18, 611-626.

DEFACTO (2002). Implementatiehandleiding - Naar de toepassing van de C-MIS.

Dempsey D.A., \& Benowitz N.L. (2001). Risks and benefits of nicotine to aid smoking cessation in pregnancy. Drug Safety, 24, 277-322.

Dijkstra, A., \& De Vries, H. (2000). Subtypes of precontemplating smokers defined by different long-term plans to change their smoking behavior. Health Education Research, 15, 423-434.

Dijkstra, R., Wensing, M., Thomas, R., Akkermans, R., Braspenning, J., Grimshaw, J., et al. (2006). The relationship between organisational characteristics and the effects of clinical guidelines on medical performance in hospitals: a meta-analysis. BioMed Central Health Services Research, 6, 53.

Emmons, K. M., \& Goldstein, M. G. (1992). Smokers who are hospitalized: a window of opportunity for cessation interventions. Preventive Medicine, 21, 262-269. 
Fahrenwald, N. L., \& Walker, S. N. (2003). Application of the Transtheoretical Model of behavior change to the physical activity behavior of WIC mothers. Public Health Nursing, 20, 307-317.

Fiore, M. C., Bailey, W. C., Cohen, S. J., Dorfman, S., Goldstein, M. G., Gritz, E. R., et al. (2000). Treating tobacco use and dependence. Clinical practice guideline. Rockville, MD: US department of Health and Human Services. Public Health Service.

Fiset, L., \& Grembowski, D. (1997). Adoption of innovative caries-control services in dental practice: a survey of Washington State dentists. Journal of the American Dental Organization, 128, 337-345.

Fleuren, M., Wiefferink, K., \& Paulussen, T. (2004). Determinants of innovation within health care organizations: literature review and Delphi study. International Journal of Quality in Health Care, 16, 107-123.

Foy, R., Eccles, M., \& Grimshaw, J. (2001). Why does primary care need more implementation research? Family Practice, 18, 353-355.

France, E. K., Glasgow, R. E., \& Marcus, A. C. (2001). Smoking cessation interventions among hospitalized patiënts: what have we learned? Preventive Medicine, 32, 376-388.

Garg, A. X., Adhikari, N. K., McDonald, H., Rosas-Arellano, M. P., Devereaux, P. J., Beyene, J., et al. (2005). Effects of computerized clinical decision support systems on practitioner performance and patient outcomes: a systematic review. Journal of the American Medical Association, 293, 1223-1238.

Glasgow R.E., Lichtenstein E., \& Marcus A.C. (2003). Why don't we see more translation of health promotion research to practice? Rethinking the efficacy-to-effectiveness transition. American Journal of Public Health, 93, 1261-7.

Glasgow, R. E., Vogt, T. M., \& Boles, S. M. (1999). Evaluating the public health impact of health promotion interventions: the RE-AIM framework. American Journal of Public Health, 89, 1322-1327.

Godin, G., \& Kok, G. (1996). The theory of planned behavior: a review of its applications to health-related behaviors. American Journal of Health Promotion, 11, 87-98.

Gollwitzer, P. M. (1999). Implementation Intentions - Strong effects of simple plans. American Psychologist, 54, 493-503.

Gollwitzer, P. M., \& Brandstätter, V. (1997). Implementation intentions and effective goal pursuit. Journal of Personality and Social Psychology, 71, 186-199.

Green, R., \& Kreuter, M. (1999). Health Promotion Planning: An Educational and Ecological Approach (3th ed ed.). Mountain View, CA: Mayfield. 
Grimshaw, J. M., Thomas, R. E., MacLennan, G., Fraser, C., Ramsay, C. R., Vale, L., et al. (2004). Effectiveness and efficiency of guideline dissemination and implementation strategies. Health Technology Assessment, 8(6), iii-iv, 1-72.

Grol, R. (2001). Successes and failures in the implementation of evidence-based guidelines for clinical practice. Medical Care, 39(8 Suppl 2), II46-54.

Grol, R., \& Grimshaw, J. (2003). From best evidence to best practice: effective implementation of change in patients' care. Lancet, 362, 1225-1230.

Grol, R., \& Wensing, M. (2001). Implementatie - effectieve verandering in de patiëntenzorg [Implementation - effective change in patient care]. Maarssen: Elsevier gezondheidszorg.

Gross P.A., Greenfield S., Cretin S., Ferguson J., Grimshaw J., Grol R., et al. (2001). Optimal methods for guideline implementation: conclusions from Leeds Castle meeting. Medical Care, 39, II85-92.

Haagen, E. C., Nelen, W. L., Hermens, R. P., Braat, D. D., Grol, R. P., \& Kremer, J. A. (2005). Barriers to physician adherence to a subfertility guideline. Human Reproduction, 20, 3301-3306.

Hage, J., \& Aiken, M. (1970). Social Change in Complex Organisations. New York: Random House.

Haines A., \& Donald A. (1998). Getting research findings into practice: Making better use of research findings. British Medical Journal, 317, 72-75.

Hajek P., West R., Lee A., Foulds J., Owen L., Eiser J.R., et al. (2001). Randomized controlled trial of a midwife-delivered brief smoking cessation intervention in pregnancy. Addiction, 96, 485-94.

Harting, J., Van Assema, P., Ruland, E., Van Limpt, P., Gorgels, T., Van Ree, J., et al. (2005). Implementation of an innovative health service a "real-world" diffusion study. American Journal of Preventive Medicine, 29, 113-119.

Holm, K., Kremers, S. P., \& De Vries, H. (2003). Why do Danish adolescents take up smoking? European Journal of Public Health, 13, 67-74.

Hulscher, M. E. J. L., Wensing, M., Van der Weijden, T., \& Grol, R. (2003). Interventions to implement prevention in primary care. Cochrane Database Systematic Reviews, CD000362.

Institute of Medicine (1990). Clinical Practice Guidelines: Directions for a New Program. Washington D.C.: National Academy Press.

Jamtvedt, G., Young, J., Kristoffersen, D., O'Brien, M., \& Oxman, A. (2006). Audit and feedback: effects on professional practice and health care outcomes. Cochrane Database Systematic Reviews, CD000259.

Janz, N. K., \& Becker, M. H. (1984). The Health Belief Model: a decade later. Health Education Quarterly, 11, 1-47. 
Kans, T. (2004). Implementatietraject V-MIS - Rapportage ten behoeve van ZonMw, Project 2500.0007. Den Haag: STIVORO voor een rookvrije toekomst.

Katz, D. A., Brown, R. B., Muehlenbruch, D. R., Fiore, M. C., \& Baker, T. B. (2004). Implementing guidelines for smoking cessation: comparing the efforts of nurses and medical assistants. American Journal Preventive Medicine, 27, 411-416.

Kenens, R., \& Hingstman, L. (2004). Cijfers uit de registratie van verloskundigen, peiling 2004 [Data from the registration of midwives, 2004 survey]. Utrecht: Nivel, Institute for Health Services Research.

Kok, G., Van den Borne, B., \& Mullen, P. D. (1997). Effectiveness of health education and health promotion: meta-analyses of effect studies and determinants of effectiveness. Patient Education and Counseling, 30, 19-27.

Koopman, P. L., \& Pool, J. (1991). Organizational decision making: Models, Contingencies and Strategies. In J. Rasmussen, B. Brehmer \& J. Leplat (Eds.), Distributed decision making: Cognitive models for cooperative work. Chichester: Wiley \& Sons.

Koopman, P. L., \& Pool, J. (1994). Chapter 6: Decision making in organizations. In C. C. Cooper \& I. T. Robertson (Eds.), Key reviews in managerial psychology: Concepts and research for practice. Chichester: Wiley \& Sons.

Kotler, P., \& Roberto, E. (1991). Sociale Marketing. Utrecht: Het Spectrum.

Lanting C.I., Van Wouwe J.P., \& Crone M.R. (2005). Roken en stoppen met roken in de periode rond de zwangerschap - Tussentijdse rapportage [Smoking and quitting around pregnancy]. Leiden: TNO Kwaliteit van Leven, pp. 1-16.

Lechner, L., \& Brug, J. (1997). Consumption of fruit and vegetables: how to motivate the population to change their behavior. Cancer Letters, 114, 335-336.

Lechner, L., De Nooijer, J., \& De Vries, H. (2004). Breast self-examination: Iongitudinal predictors of intention and subsequent behaviour. European Journal of Cancer Prevention, 13, 369-376.

Lippke, S., Ziegelmann, J. P., \& Schwarzer, R. (2004). Behavioral intentions and action plans promote physical exercise: A longitudinal study with orthopedic rehabilitation patients. Journal of Sport \& Exercise Psychology, 26, 470-483.

Locke, E. A., \& Latham, G. P. (2002). Building a practically useful theory of goal setting and task motivation. A 35-year odyssey. American Psychologist, 57, 705-717.

Lumley, J., Oliver, S. S., Chamberlain, C., \& Oakley, L. (2004). Interventions for promoting smoking cessation during pregnancy. Cochrane Database of Systematic Reviews, CD001055.

McCarty, M. C., Hennrikus, D. J., Lando, H. A., \& Vessey, J. T. (2001). Nurses' attitudes concerning the delivery of brief cessation advice to hospitalized smokers. Preventive Medicine, 33, 674-681. 
Mesters I., \& Meertens R.M. (1999). Monitoring the dissemination of an educational protocol on pediatric asthma in family practice: a test of associations between dissemination variables. Health Education and Behavior, 26, 103-20.

Michie S., Hendy J., Smith J., \& Adshead F. (2004). Evidence into practice: a theory based study of achieving national health targets in primary care. Journal of Evaluation in Clinical Practice, 10, 447-56.

Mittman, B. S., Tonesk, X., \& Jacobson, P. D. (1992). Implementing clinical practice guidelines: social influence strategies and practitioner behavior change. Quality Review Bulletin, 18, 413-422.

Moch, M. K., \& Morse, E. V. (1977). Size, centralization and organizational adoption of innovations. American Sociological Review, 42, 716-725.

Moore L., Campbell R., Whelan A., Mills N., Lupton P., Misselbrook E., et al. (2002). Self help smoking cessation in pregnancy: cluster randomised controlled trial. British Medical Journal, 325, 1383-1388.

Mudde A., Willemsen M.C., Kremers S., \& De Vries H. (2000). Meetinstrumenten - voor onderzoek naar roken en stoppen met roken [Measurement tools for research in smoking cessation]. Den Haag: Stivoro.

Nagelkerke, N. J. D. (1991). A note on a general definition of the coefficient of determinantion. Biometrika, 78, 691-692.

Narsavage, G., \& Idemoto, B. K. (2003). Smoking cessation interventions for hospitalized patients with cardio-pulmonary disorders. Online Journal of Issues in Nursing, $8(2), 8$.

NIVEL (2004). Cijfers uit de registratie van verloskundigen, peiling 2004 [Data from the midwives registry, 2004 survey]. Utrecht: Nivel.

NIVEL (1996). Cijfers uit de registratie van huisartsen, peiling 1996 [Data from the registration of general practitioners, survey 1996]. Utrecht: Nivel Institute for Health Services Research.

NIVEL (2005). Cijfers uit de registratie van verloskundigen, peiling 2005 [Data from the registration of midwives, 2005 survey]. Utrecht: Nivel Institute for Health Services Research.

Norman, P., \& Conner, M. (2006). The theory of planned behaviour and binge drinking: Assessing the moderating role of past behaviour within the theory of planned behaviour. British Journal of Health Psychology, 11, 55-70.

NVHVV (2003). Personal communication Mrs. Carmen Cornelia of the NVHVV (Dutch Federation of Cardiovascular Nurses).

Oldenburg, B. F., Sallis, J. F., Ffrench, M. L., \& Owen, N. (1999). Health promotion research and the diffusion and institutionalization of interventions. Health Education Research, 14, 121-130. 
Orlandi, M. A. (1987). Promoting health and preventing disease in health care settings: an analysis of barriers. Preventive Medicine, 16, 119-130.

Orleans, C. T., Rotberg, H. L., Quade, D., \& Lees, P. (1990). A hospital quit-smoking consult service: clinical report and intervention guidelines. Preventive Medicine, $19,198-212$.

Ovretveit, J. (1999). A team quality improvement sequence for complex problems. Quality in Health Care, 8, 239-246.

Parcel, G. S., O'Hara-Tompkins, N. M., Harrist, R. B., Basen-Engquist, K. M., McCormick, L. K., Gottlieb, N. H., et al. (1995). Diffusion of an effective tobacco prevention program. Part II: Evaluation of the adoption phase. Health Education Research, 10, 297-307.

Park, E. R., DePue, J. D., Goldstein, M. G., Niaura, R., Harlow, L. L., Willey, C., et al. (2003). Assessing the transtheoretical model of change constructs for physicians counseling smokers. Annals of Behavioral Medicine, 25, 120-126.

Paulussen, T., Kok, G., \& Schaalma, H. (1994). Antecedents to adoption of classroombased AIDS education in secondary schools. Health Education Research, 9, 485496.

Paulussen, T., Kok, G., Schaalma, H., \& Parcel, G. S. (1995). Diffusion of AIDS curricula among Dutch secondary school teachers. Health Education Quarterly, 22, 227243.

Pieterse, M. E. (1999). Stoppen met roken met hulp van de huisartspraktijk [Smoking cessation with general practitioners'help]. PhD Thesis University of Twente, Enschede.

Pieterse, M. E., Seydel, E. R., DeVries, H., Mudde, A. N., \& Kok, G. J. (2001). Effectiveness of a minimal contact smoking cessation program for Dutch general practitioners: a randomized controlled trial. Preventive Medicine, 32, 182-190.

Pieterse. M.E. (2001). De parallelle implementatie van de Minimale Interventiestrategie onder 3 beroepsgroepen, gericht op stoppen met roken bij risicogroepen: evaluatie van een regionaal project rond de C-MIS en de V-MIS in Nijmegen en omstreken [Evaluation of a Regional Project on the Implementation of Minimal Intervention Strategies for Smoking Cessation]. Nijmegen, The Netherlands: GGD.

Pool, J., \& Koopman, P. L. (1992). Strategic decision making in organizations - A research model and some initial findings. In D. M. Mosking \& N. Anderson (Eds.), Organizational change and innovation: Psychological perspectives and practices in Europe. London: Rontledge.

Prochaska, J. O., \& DiClemente, C. C. (1983). Stages and processes of self-change of smoking: toward an integrative model of change. Journal of Consulting and Clinical Psychology, 51, 390-395. 
Prochaska, J. O., \& Velicer, W. F. (1997). The transtheoretical model of health behavior change. American Journal of Health Promotion, 12, 38-48.

Pullon, S., McLeod, D., Benn, C., Viccars, A., White, S., Cookson, T., et al. (2003). Smoking cessation in New Zealand: education and resources for use by midwives for women who smoke during pregnancy. Health Promotion International, 18, 315-325.

Quist-Paulsen, P., \& Gallefoss, F. (2003). Randomised controlled trial of smoking cessation intervention after admission for coronary heart disease. British Medical Journal, 327, 1254-1257.

Raw, M., McNeill, A., \& West, M. A. (1998). About these guidelines. Thorax, 53(Suppl. 5), S1-S19.

Raw, M., McNeill, A., \& West, R. (1999). Smoking cessation: evidence based recommendations for the healthcare system. British Medical Journal, 318, 182185.

Riemsma, R. P., Pattenden, J., Bridle, C., Sowden, A. J., Mather, L., Watt, I. S., et al. (2003). Systematic review of the effectiveness of stage based interventions to promote smoking cessation. British Medical Journal, 326, 1175-1177.

Rigotti, N. A., Munafo, M. R., Murphy, M. F., \& Stead, L. F. (2003). Interventions for smoking cessation in hospitalised patients. Cochrane Database Systematic Reviews, CD001837.

Rogers, E. M. (1995). Diffusion of innovations (4 ed.). New York: The Free Press.

Rogers, E. M. (2002). Diffusion of preventive innovations. Addictive Behaviors, 27, 989993.

Roovers, M. (2005). Implementatietraject V-MIS - Rapportage ten behoeve van ZonMw, Project 2500.0007 [Implementation report V-MIS]. Den Haag: STIVORO voor een rookvrije toekomst.

Sacker, A. (1990). Smoking habits of nurses and midwives. Journal of Advanced Nursing, $15,1341-1346$.

Scanlon, P. D., Connett, J. E., Waller, L. A., Altose, M. D., Bailey, W. C., \& Buist, A. S. (2000). Smoking cessation and lung function in mild-to-moderate chronic obstructive pulmonary disease. The Lung Health Study. American Journal of Respiratory and Critical Care Medicine, 161, 381-390.

Scholte Op Reimer, W., De Swart, E., De Bacquer, D., Pyorala, K., Keil, U., Heidrich, J., et al. (2006). Smoking behaviour in European patients with established coronary heart disease. European Heart Journal, 27, 35-41.

Schuster, M. A., McGlynn, E. A., \& Brook, R. H. (2005). How good is the quality of health care in the United States? Milbank Quaterly, 83(4), 843-895. 
Seddon, M. E., Marshall, M. N., Campbell, S. M., \& Roland, M. O. (2001). Systematic review of studies of quality of clinical care in general practice in the UK, Australia and New Zealand. Quality in Health Care, 10, 152-158.

Segaar, D., \& Willemsen, M. C. (2003). Tabaksrook in de werkomgeving - Resultaten van het PARA-meter onderzoek onder werknemers (Tobacco Smoke in the Workplace Result of a research among employees). The Hague: STIVORO - for a smokefree future.

Segaar, D., \& Willemsen, M. C. (2004). Samenvatting evaluatie C-MIS [Summary of evaluation of the Minimal Intervention Strategy on Smoking Cessation for Cardiac wards]. The Hague: STIVORO.

Segaar, D., \& Willemsen, M. C. (2005). Samenvatting evaluatie V-MIS [Summary of evaluation of the Minimal Intervention Strategy on Smoking Cessation for Midwives]. The Hague: STIVORO.

Segaar, D., Bolman, C., Willemsen, M. C., \& De Vries, H. (2006). Determinants of adoption of cognitive behavioral interventions in a hospital setting: Example of a minimal-contact smoking cessation intervention for cardiology wards. Patient Education and Counseling, 61, 262-271

Segaar, D., Bolman, C., Willemsen, M. C., \& De Vries, H. (in press). Identifying determinants of protocol adoption by midwives: A comprehensive approach. Health Education Research, in press.

Segaar, D., Bolman, C., Willemsen, M. C., \& De Vries, H. (submitted). Adherence to a Minimal-Contact Smoking Cessation Intervention by Midwives.

Sheeran, P. (2002). Intention-behaviour relations: A conceptual and empirical review. In W. Stroebe \& M. Hewstone (Eds.), European review of social psychology, 12, 136. London: Wiley.

Sheeran, P., \& Abraham, C. (1995). The Health Belief Model. In M. Conner \& P. Norman (Eds.), Predicting Health Behaviour (pp. 230). Buckingham: Open University Press.

Shekelle, P. G., Woolf, S. H., Eccles, M., \& Grimshaw, J. (1999). Clinical guidelines: developing guidelines. British Medical Journal, 318, 593-596.

Sherrill, D. L., Holberg, C. J., Enright, P. L., Lebowitz, M. D., \& Burrows, B. (1994). Longitudinal analysis of the effects of smoking onset and cessation on pulmonary function. American Journal of Respiratory and Critical Care Medicine, 149, 591597.

Sinclair, R. C., Maxfield, A., Marks, E. L., Thompson, D. R., \& Gershon, R. R. (2002). Prevalence of safer needle devices and factors associated with their adoption: results of a national hospital survey. Public Health Reports, 117, 340-349. 
Sniehotta, F. F., Scholz, U., \& Schwarzer, R. (2005). Bridging the intention-behaviour gap: Planning, self-efficacy, and action control in the adoption and maintenance of physical exercise. Psychology and Health, 20, 143-160.

Steckler, A., Goodman, R. M., McLeroy, K. R., Davis, S., \& Koch, G. (1992). Measuring the diffusion of innovative health promotion programs. American Journal of Health Promotion, 6, 214-224.

Stoline, M. R. (1981). The status of multiple comparisins: Simultaneaous estimation of all pairwise comparisons in one-way anova designs. The American Statistician, 35, 134-141.

Tabachnick, B., \& Fidell, L. (2001). Using multivariate Statistics (4th ed.). Boston: Allyn and Bacon.

Taylor, C. B., Houston-Miller, N., Killen, J. D., \& DeBusk, R. F. (1990). Smoking cessation after acute myocardial infarction: effects of a nurse-managed intervention. Annals of Internal Medicine, 113, 118-123.

Thomson O'Brien, M. A., Freemantle, N., Oxman, A. D., Wolf, F., Davis, D. A., \& Herrin, J. (2001). Continuing education meetings and workshops: effects on professional practice and health care outcomes. Cochrane Database of Systematic Reviews, CD003030.

Thomson O'Brien, M. A., Oxman, A. D., Haynes, R. B., Davis, D. A., Freemantle, N., \& Harvey, E. L. (2000). Local opinion leaders: effects on professional practice and health care outcomes. Cochrane Database of Systematic Reviews, CD000125.

TNO Preventie en Gezondheid (2000). De thuisbevalling in Nederland. Eindrapportage: 1995-2000 [Home delivery in the Netherlands. Final Report 1995-2000]. Leiden: TNO Preventie en Gezondheid.

TNS NIPO (2002). Roken en zwangerschap [Smoking and pregnancy]. Den Haag: DEFACTO voor een rookvrije toekomst.

TNS NIPO (2005). Continu onderzoek rookgewoonten 2004. [Continuous monitoring of smoking habits]. Den Haag: STIVORO voor een rookvrije toekomst.

Tweede Kamer der Staten Generaal (2005). Vaststelling van de begrotingsstaten van het Ministerie van Volksgezondheid, Welzijn en Sport (XVI) voor het jaar 2006 [Budget Ministery of Health]. Den Haag: Tweede Kwamer der Staten Generaal.

Tsiara, S., Elisaf, M., \& Mikhailidis, D.P. (2003). Influence of smoking on predictors of vascular disease. Angiology, 54, 507-530.

US Department of Health and Human Services (2004). The Health Consequences of Smoking: A Report of the Surgeon General. Atlanta, Ga: US Department of Health and Human Services, CDC, National Center for Chronic Disease Prevention and Health Promotion, Office on Smoking and Health. 
US Department of Health and Human Services (2001). Woman and Smoking- A report of the Surgeon General. Rockville, MD: US Department of Health and Human Services.

Van Weel C., Coebergh J.W., Drenthen T., Schippers G.M., Van Spiegel P.I., Anderson P.D., et al. (2005). Richtlijn 'Behandeling van tabaksverslaving' [The practice guideline: 'Treatment of tobacco dependence']. Nederlands Tijdschrift voor Geneeskunde, 149, 17-21.

Vaughn, T. E., McCoy, K. D., BootsMiller, B. J., Woolson, R. F., Sorofman, B., TrippReimer, T., et al. (2002). Organizational predictors of adherence to ambulatory care screening guidelines. Medical Care, 40, 1172-1185.

Vermette, L., \& Godin, G. (1996). Nurses' intentions to provide home care: the impact of AIDS and homosexuality. AIDS Care, 8, 479-488.

Webb, T. L., \& Sheeran, P. (2006). Does changing behavioral intentions engender behavior change? A meta-analysis of the experimental evidence. Psychological Bulletin, 132, 249-268.

Weinstein N.D. (1988). The precaution adoption process. Health Psychology, 7, 355-86.

Wiegers, T., \& Van Wieren, S. (2005). Verloskundige zorg samengevat [Midwifery care summarized] In: Volksgezondheid Toekomst Verkenning. Bilthoven: RIVM.

Wiggers, L. C., Oort, F. J., Dijkstra, A., De Haes, J. C., Legemate, D. A., \& Smets, E. M. (2005). Cognitive changes in cardiovascular patients following a tailored behavioral smoking cessation intervention. Preventive Medicine, 40, 812-821.

Wilhelm, S. L. (2002). Factors affecting a woman's intent to adopt hormone replacement therapy for menopause. Journal of Obstetric Gynecologic and Neonatal Nursing, 31, 698-707.

Willemsen, M. C., Meijer, A., \& Jannink, M. (1999). Applying a contingency model of strategic decision making to the implementation of smoking bans: a case study. Health Education Research, 14, 519-531.

Zaltman, G., Duncan, R., \& Holbek, J. (1973). Innovations and Organizations. New York: Wiley. 



\section{Summary}

Smoking causes high health risks. Health care professionals frequently have to deal with smoking patients. Several interventions for smoking cessation support by health professionals are available that effectively increase the chance that a smoker successfully quits smoking. Yet, a substantial part of the potential effect of those interventions is lost due to non-use or improper use of the intervention.

Between 2000 and 2004 STIVORO carried out programs for national diffusion of the effective Minimal Intervention strategies for Smoking cessation support (MIS) on cardiac wards (C-MIS) and in midwifery practices (V-MIS). This thesis describes studies that are conducted during or right after this period to assess the level and quality of use of this intervention and to identify factors that facilitated or impeded successful adoption (getting familiar with the intervention and forming a positive attitude towards it) and implementation (putting the intervention into use as intended) of the intervention. The comprehensive theoretical framework that was used for identifying potentially important factors is the Integrated Change or I-Change Model. The applicability of this model in diffusion research will be tested in this thesis. Chapter 1 gives a more extensive general introduction to the background of this thesis and describes the I-Change Model.

Chapter 2 describes the results of a cross-sectional study among the heads of all 121 cardiology wards in Dutch hospitals, of whom 77 (64\%) responded. The main aim of the study was to identify the factors that were associated with adoption of the C-MIS on cardiac inpatient wards. The study was performed 4 years after the first nationwide introduction of the C-MIS. At that time $63 \%$ of the participating wards had adopted the MIS. The study showed that wards that adopted the C-MIS were on the average less formalized and that generally there were more nurses working on those wards. Heads of wards that adopted the C-MIS had a more positive attitude towards working with the CMIS, they perceived more positive social influences from their direct environment and they perceived higher self-efficacy. Generally, personal awareness and motivational factors were significantly stronger associated with adoption of the C-MIS than organizational factors.

Chapter 3 presents the results of a cross-sectional study among cardiac nurses from wards that decided to use the C-MIS. A random sample of 25 wards was taken from the 39 wards that decided to use the C-MIS, 20 wards ( $80 \%)$ agreed to participate. These wards employed 553 cardiac nurses, of whom 210 (38\%) responded to our questionnaire. The study assessed the level and adherence of use of the C-MIS and aimed to identify the factors that were associated with adherent use of the C-MIS by cardiac nurses. Adherent and nonadherent users of the C-MIS were compared. Forty-six percent of the nurses did not manage to fully adhere to the C-MIS in daily practice; they did not always provide patients with self-help guides, discuss smoking cessation aids 
and/or provide follow-up care. Factors in the I-Change Model were associated with 52\% of the variance in adherence. Adherence was most likely if nurses consistently used an intervention report form that showed all steps of the C-MIS (the so-called intervention card), perceived many advantages of using the C-MIS, had many other nurses around them who used the C-MIS, worked on a ward that had no designated smoking facilities for nurses and involved nurses in decision-making. Generally psychosocial variables were much stronger associated with adherence to the C-MIS than organizational variables.

Chapter 4 describes a cross-sectional study among all 446 Dutch midwifery practices, of which 251 (56\%) responded. The study was performed 2 years after the first nationwide introduction of the MIS for midwives (V-MIS). At that time $36 \%$ of the responding practices used the V-MIS, $17 \%$ was planning to use the V-MIS in the near future (intenders) and $47 \%$ was not planning to use the V-MIS (non-intenders). Adoption of the MIS protocol was facilitated by the presence of practice assistants and impeded by having a large proportion of clients of foreign origin. Yet, personal awareness and motivational factors (such as knowledge about the V-MIS, personal relevance of smoking cessation support of pregnant women, perceived advantages and disadvantages and perceived social norms) were more strongly associated with adoption of the V-MIS by midwifery practices than organizational factors were. For midwives, the most important information channel on the V-MIS was the midwives' professional organization (KNOV).

Chapter 5 presents the results of a cross-sectional study among a random sample of 250 midwives who were trained in using the V-MIS, of whom 137 (55\%) responded. This chapter reports on the adherence to MIS by midwives and identifies psychosocial and organizational factors that were associated with adherence to the protocol. Thirtythree midwives indicated they never actually started to use the V-MIS (24\%), 37 midwives (27\%) used the V-MIS, but turned out not perform the steps of the V-MIS consistently and 66 midwives (48\%) adherently used the V-MIS. One midwife did not fill in the questionnaire properly and was therefore excluded from further analyses. If midwives used the V-MIS they almost always asked their clients about their smoking behavior, advised them to quit smoking, assessed their motivation and discussed the reasons to quit. V-MIS elements that were less consistently applied included discussing barriers to smoking cessation, setting a quit date with motivated clients and providing follow-up care. Factors in the I-Change Model were associated with $56 \%$ of the variance in adherence. Adherence was most likely if midwives had the opinion that smoking cessation support of pregnant women is important, if they were innovative in nature, if they perceived much social support, if they had sufficient skills to perform all steps of the V-MIS protocol and if they used an intervention report form that showed all steps of the MIS (the intervention card). 
Chapter 6 describes the results of a longitudinal study among midwives after the organizational and psychosocial determinants of initiation of implementation of the MIS by midwifery practices. In this study, for all practices that were non-adopters at the time of the study described in chapter 4, information was gathered on whether they started to implement the MIS one year later. This was the case if at least one midwife in the practice attended the skills training and the V-MIS educational materials were ordered. The results show that implementation was more likely in practices with more knowledge on the intervention, with stronger intentions to implement it, with greater dissatisfaction with the way of working and in practices that employed more midwives. These factors together predicted $31 \%$ of the variance in implementation.

In chapter 7 a comparison is made between the results of the four studies that are described in chapter 2 to 5 with respect to factors associated with adoption or implementation. Factors that are strongly associated with adoption of the V-MIS are compared to factors that are strongly associated with implementation of the MIS and it is assessed whether those factors agree or differ between midwifery practices and cardiac wards. Results showed that smoking behavior, level of centralization, proportion of external versus internal information sources used, and workload were not significantly associated with either adoption or adherence in both settings. Knowledge, perceived advantages, perceived disadvantages, social norms and social pressure were significantly associated with adoption and successful implementation in both settings. Several other variables were only significantly associated with either adoption or successful implementation or they were significantly associated with both diffusion stages but only in one of the two settings.

Chapter 8 summarizes the results of the former chapters and puts them into perspective as far as not already done in chapter 7 . Three general conclusions are drawn. First, using the MIS could yield considerable gains in terms of extra quitters, if implementation rates and the quality of use could be increased. Second, the factors that were strongly associated with adoption differed from the factors that were strongly associated with implementation. To increase the number of health professionals that use the MIS, adoption strategies should preferably focus on increasing motivation and intention by increasing knowledge on the MIS, increasing perceived advantages, decreasing perceived disadvantages and increasing the perceived positive norms and support in the social environment. Adherent use of the MIS could be increased by increasing the skills of professionals to perform all steps of the MIS and by stimulating use of an intervention report form that showed all steps of the MIS (the intervention card). Organizational factors must be taken into account when developing adoption and implementation strategies, but play a smaller role than motivational factors. At last, our studies indicate that the I-Change Model can be a useful framework for diffusion 
research, when studying individuals within organizations, and that all factors identified in this model, i.e. predisposing, information, awareness, motivational and ability factors, contribute to the final behavior. However, because the model was tested crosssectionally in most of our studies, it will be useful to perform more longitudinal studies to verify the order and patterns assumed in the model. Furthermore, our studies indicate that when the I-Change Model is used in diffusion research there is room for some improvements and extensions. Chapter 8 ends with conclusions and recommendations for future research and for practice. 


\section{Samenvatting}

Zorgverleners hebben in hun dagelijkse praktijk regelmatig te maken met patiënten die roken, met daardoor een hoger ziekte- of sterfterisico. Zorgverleners kunnen een belangrijke rol spelen in de begeleiding bij stoppen met roken. Er zijn verscheidene interventies die zorgverleners daarvoor kunnen gebruiken, waarvan is bewezen dat ze de kans dat een roker succesvol stopt met roken significant verhogen. Echter, een groot deel van het potentiële effect van deze interventies op de volksgezondheid gaat verloren doordat zorgverleners de interventies niet of niet op de juiste manier gebruiken.

Tussen 2000 en 2004 heeft STIVORO zich bezig gehouden met het invoeren van de bewezen effectieve Minimale Interventiestrategie Stoppen-met-roken (MIS) op verpleegafdelingen cardiologie (C-MIS) en in verloskundigenpraktijken (V-MIS). Dit proefschrift beschrijft onderzoeken die gedurende en vlak na deze periode zijn uitgevoerd om de mate en kwaliteit van het gebruik van de C-MIS en de V-MIS in de praktijk te peilen en de factoren te identificeren die succesvolle adoptie (het vormen van een positieve houding ten aanzien van de interventie) en implementatie (het daadwerkelijk uitvoeren van de interventie) in de praktijk stimuleren of belemmeren. Voor de identificatie van mogelijke beïnvloedende factoren is gebruik gemaakt van een uitgebreid onderzoeksmodel voor het verklaren van gedragsverandering; het Integrated Change of I-Change Model. De toepasbaarheid van dit model in diffusieonderzoek wordt in dit proefschrift getest. Hoofdstuk 1 geeft een uitgebreidere achtergrond van dit proefschrift weer en beschrijft het I-Change Model.

Hoofdstuk 2 beschrijft de resultaten van een cross-sectionele studie, waarbij de verpleegkundig hoofden van alle 121 cardiologie verpleegafdelingen in Nederlandse ziekenhuizen werden benaderd met een vragenlijst. Zevenenzeventig (64\%) van hen retourneerden de ingevulde vragenlijst. Het belangrijkste doel van de studie was het identificeren van factoren die samenhangen met adoptie van de C-MIS op verpleegafdelingen cardiologie. De studie vond vier jaar na de eerste landelijke presentatie van de C-MIS plaats. Op dat moment had $63 \%$ van de deelnemende afdelingen de C-MIS geadopteerd. De studie liet zien dat de besluitvorming op afdelingen die de C-MIS hadden geadopteerd gemiddeld minder via vaste procedures plaatsvond en dat er over het algemeen meer verpleegkun-digen op deze afdelingen werkten. Hoofden van afdelingen die de C-MIS hadden geadopteerd hadden een positievere attitude ten aanzien van het werken met de C-MIS, ze verwachtten meer positieve sociale invloed van hun directe omgeving en ze hadden hogere eigen-effectiviteitsverwachtingen. Over het algemeen bleken bewustzijns- en motivatiefactoren sterker samen te hangen met adoptie dan organisatiefactoren.

Hoofdstuk 3 presenteert de resultaten van een cross-sectionele studie onder verpleegkundigen die werkzaam waren op een verpleegafdeling cardiologie waar was 
besloten de C-MIS te gebruiken. Uit de 39 afdelingen die ten tijden van het onderzoek aan deze voorwaarde voldeden werd een gerandomiseerde steekproef getrokken van 25 afdelingen die werden benaderd om aan het onderzoek mee te werken, 20 afdelingen (80\%) gingen akkoord. Op deze afdelingen werkten 553 hartverpleegkundigen, van wie er 210 (38\%) onze vragenlijst invulden. De studie bekeek de mate waarin verpleegkundigen de stappen van de C-MIS, zoals omschreven in het protocol, volledig en consequent uitvoerden. Vervolgens werd gekeken welke factoren geassocieerd waren met het al dan niet volledig en consequent uitvoeren van alle stappen van de C-MIS, door de "gedegen" en de "minder gedegen" gebruikers van de C-MIS met elkaar te vergelijken. Van de verpleegkundigen die de C-MIS gebruikten bleek $46 \%$ er niet in te slagen het C-MIS protocol volledig en consequent toe te passen; ze gaven hun patiënten niet altijd een zelfhulpgids, ze spraken niet altijd over stoppen met roken hulpmiddelen en/of ze gaven niet altijd nazorg. Factoren in het I-Change Model waren geassocieerd met $52 \%$ van de variantie in de "gedegenheid" waarmee het protocol werd uitgevoerd. Verpleegkundigen bleken het protocol het meest volledig en consequent te gebruiken als ze gebruik maakten van een kaart waarop alle stappen van de C-MIS stonden aangeduid en warop ze de uitgevoerde onderdelen van de C-MIS konden registreren (de zgn. interventiekaart), als ze veel voordelen zagen van het gebruik van de C-MIS, als veel verpleegkundigen in hun omgeving de C-MIS ook gebruikten en als ze betrokken waren geweest bij de besluitvorming over het gebruik van de C-MIS op de afdeling. De aanwezigheid van een ruimte op de afdeling zelf waar verpleegkundigen mochten roken werkte belemmerend. Over het algemeen gold dat psychosociale factoren sterker samenhingen met volledig en consequent gebruik van de C-MIS dan organisatiefactoren.

Hoofdstuk 4 beschrijft een cross-sectionele studie, waarvoor alle 446 verloskundigenpraktijken in Nederland zijn benaderd. Van 251 (56\%) praktijken vulde een vertegenwoordiger onze vragenlijst in. De studie vond twee jaar na de eerste landelijke introductie van de V-MIS plaats. Op dat moment gebruikte $36 \%$ van de praktijken die aan het onderzoek deelnamen de V-MIS, $17 \%$ was van plan om de V-MIS op korte termijn te gaan gebruiken en $47 \%$ was niet van plan om de V-MIS te gaan gebruiken. Praktijken waren eerder geneigd om de V-MIS te gaan gebruiken indien er praktijkassistenten beschikbaar waren en minder geneigd indien de praktijk veel allochtone cliënten had. Echter, bewustzijnsfactoren en motivatie factoren (zoals kennis over de V-MIS, belang dat men hecht aan stoppen-met-roken begeleiding van zwangeren, verwachte voor- en nadelen en verwachte positieve normen in de sociale omgeving) bleken sterker samen te hangen met het adopteren van de V-MIS door praktijken dan organisatiekenmerken. De beroepsorganisatie van verloskundigen (KNOV) was voor verloskundigen de belangrijkste bron van informatie over de V-MIS.

Hoofdstuk 5 presenteert de resultaten van een cross-sectionele studie onder een 
gerandomiseerde steekproef van 250 verloskundigen die waren getraind in het toepassen van de V-MIS. In de studie werd gekeken in welke mate verloskundigen de stappen van de V-MIS consequent en volledig toepasten en welke factoren samenhingen met het consequente en volledige gebruik van de V-MIS. Van de benaderde verloskundigen vulden er 137 (55\%) onze vragenlijst in. Drieëndertig van deze verloskundigen (24\%) gaven aan de V-MIS nooit te zijn gaan gebruiken, 37 verloskundigen (27\%) gebruikten de V-MIS, maar bleken de stappen van de V-MIS niet consequent en volledig uit te voeren en 66 verloskundigen (48\%) gebruikten de V-MIS consequent en volledig. Eén verloskundige vulde de vragenlijst zeer onvolledig in en is daarom buiten beschouwing gelaten. Indien verloskundigen de V-MIS gebruikten informeerden ze vrijwel altijd naar het rookgedrag van hun cliënten, adviseerden ze rokende cliënten vrijwel altijd om te stoppen met roken, inventariseerden ze vrijwel altijd de motivatie van cliënten om te stoppen met roken en besproken ze redenen om te stoppen met roken vrijwel altijd. Onderdelen van de V-MIS die minder consequent werden toegepast waren het bespreken van barrières voor het stoppen met roken, het vastleggen van een stopdatum indien een cliënt gemotiveerd was om te stoppen en het uitvoeren van nazorg na de stopdatum. Factoren in het I-Change Model waren geassocieerd met $56 \%$ van de variantie in gedegenheid waarmee de V-MIS werd toegepast. Verloskundigen waren het sterkst geneigd om de V-MIS consequent uit te voeren wanneer ze stoproken begeleiding aan zwangeren belangrijk vonden, ze innovatief waren ingesteld, ze veel steun ervoeren vanuit hun sociale omgeving, ze voldoende vaardigheden beschikten en wanneer ze gebruik maakten van een kaart waarop alle stappen van de V-MIS stonden aangeduid en waarop ze de uitgevoerde onderdelen konden registreren (de interventiekaart).

Hoofdstuk 6 beschrijft de resultaten van een longitudinale studie onder verloskundigenpraktijken naar de organisatie- en psychosociale factoren die bepalen of een praktijk het implementatietraject van de V-MIS in gang zet. In deze studie is voor alle praktijken die de V-MIS nog niet hadden geadopteerd ten tijden van de studie die in hoofdstuk 4 is beschreven, nagegaan of ze één jaar later het implementatietraject van de V-MIS in gang hadden gezet. Dat hield in dat minimaal één verloskundige in de praktijk de vaardighedentraining had gevolgd en dat de bij de V-MIS behorende voorlichtingsmaterialen waren besteld. De kans dat praktijken de V-MIS binnen een jaar implementeerden bleek het grootst te zijn wanneer praktijken veel kennis hadden over de V-MIS, wanneer ze sterk van plan waren om de V-MIS te implementeren, wanneer ze ontevreden waren over hun manier van werken en wanneer de praktijk uit een groot aantal verloskundigen bestond. Deze factoren samen voorspelden $31 \%$ van de variantie in implementatie.

In hoofdstuk 7 zijn de resultaten van de vier studies die zijn beschreven in hoofdstuk $2 \mathrm{t} / \mathrm{m} 5$ vergeleken wat betreft factoren die samenhangen met adoptie of 
implementatie. $\mathrm{Er}$ is gekeken in hoeverre de factoren die sterk samenhangen met adoptie overeenkomen met de factoren die sterk samenhangen met implementatie en in hoeverre daarin verschillen bestaan tussen verloskundigenpraktijken en cardiologieafdelingen. In geen van de twee settings blijken het rookgedrag van de zorgverlener, de mate waarin de besluitvorming centraal geregeld is, de mate waarin gebruik wordt gemaakt van externe informatiebronnen en de werkdruk significant samen te hangen met adoptie of implementatie. Kennis, verwachte voor- en nadelen en verwachte normen en steun in de sociale omgeving hingen sterk samen met adoptie én implementatie, zowel in verloskundigenpraktijken als op verpleegafdelingen cardiologie. Verscheidene andere variabelen hingen alleen samen met adoptie óf alleen met implementatie óf met beiden, maar dan slechts in één van de twee onderzochte settings.

In hoofdstuk 8 zijn de resultaten van de voorgaande hoofdstukken samengevat en in perspectief geplaatst voor zover dat nog niet was gebeurd in hoofdstuk 7. Er zijn drie algemene conclusies getrokken. Ten eerste blijkt er nog een hoop winst te behalen in het gebruik van de MIS, zowel door het vergroten van het aantal verloskundigen en hartverpleegkundigen dat de MIS toepast als door het verbeteren van de consequentheid en volledigheid waarmee de MIS wordt toegepast. Ten tweede blijken factoren die een grote rol spelen bij adoptie te verschillen van de factoren die een grote rol spelen bij implementatie. Om het aantal zorgverleners dat de MIS gebruikt te vergroten kunnen het beste adoptiestrategieën worden gebruikt die zich richten op het verhogen van de motivatie en intentie. Dit kan bijvoorbeeld door het vergroten van kennis over de werkwijze, het versterken van de verwachte voordelen van de MIS, het verminderen van de verwachte nadelen van de MIS en het vergroten van verwachte positieve normen en steun in de sociale omgeving. Voor het verbeteren van de toepassing van de MIS is het erg belangrijk om ervoor te zorgen dat zorgverleners voldoende vaardigheden hebben om alle onderdelen van de MIS toe te passen en vormt het gebruik van een kaart waarop alle stappen van de MIS staan aangeduid en waarop de uitgevoerde onderdelen van de MIS kunnen worden geregistreerd (interventiekaart) een belangrijk hulpmiddel. Organisatiespecifieke kenmerken dienen in ogenschouw genomen te worden maar spelen een minder grote rol dan motivatie kenmerken. Tenslotte blijkt dat het I-Change Model een bruikbaar raamwerk kan vormen voor diffusieonderzoek dat zich richt op het gedrag van individuen in een organisatie, en dat alle factoren in dit model, namelijk predisponerende-, informatie-, bewustzijns-, motivatie- en bekwaamheidsfactoren in meer of mindere mate bijdragen aan het uiteindelijke gedrag. Longitudinaal onderzoek is echter nodig om de volgorde en patronen die in het model worden verondersteld te verifiëren. Ook is er ruimte voor verbetering en uitbreiding van het model voor toepassing in diffusieonderzoek. Hoofdstuk 8 eindigt met conclusies en aanbevelingen voor toekomstig onderzoek en voor de praktijk. 


\section{Dankwoord}

Bij het doen van onderzoek en het tot stand komen van een proefschrift ben je onmiskenbaar afhankelijk van de mensen in je omgeving. Daarom is het fijn om die mensen nog eens extra op hun waarde te wijzen met een groot DANK JULLIE WEL!

In de eerste plaats wil ik mijn promotor en co-promotoren bedanken. Hein, de uren in de trein van Maastricht naar het "westen" heb ik dikwijls nodig gehad om je inspirerende opmerkingen en ideeën ten volste tot me door te laten dringen. Je optimisme en ambitieuze planningen hebben me gemotiveerd om het beste uit mezelf te halen. Onvoorstelbaar hoe je in staat was mijn stemming af te lezen uit de kwaliteit van de stukken die ik schreef. Catherine, je snelle reacties als ik met vragen of met te beoordelen stukken kwam hielpen me gemotiveerd en in mijn ritme blijven en gaven me het gevoel dat er in geval van nood of moeilijkheden altijd iemand was waar ik op terug kon vallen. Marc, bedankt voor al je prikkelende vragen, onze discussies en natuurlijk voor het zijn van een leuke dagelijkse collega en baas.

Verder gaat mijn dank uit naar alle verloskundigen en verpleegkundigen die aan mijn onderzoeken hebben meegewerkt. Zonder jullie hulp was dit boekje er niet geweest. Datzelfde geldt voor de mensen die als projectleider van de MIS-sen bezig zijn geweest met het implementeren van de interventies: Ingrid, Marleen, Tessel en Mijke. En voor de vele andere betrokkenen, zoals de mensen uit Maastricht.

STIVORO wil ik bedanken voor het bieden van de mogelijkheid om deze missie te beginnen en te voltooien, zowel door financiële steun als door het bieden van een prettige werkplek. Fleur, Ingrid en Mijke bedankt dat jullie hebben willen kijken of mijn teksten ook voor "niet-onderzoeksmensen" begrijpelijk waren en voor jullie waardevolle tips om dingen aan te passen. Ook de andere collega's en ex-collega's bij STIVORO bedankt voor de gezelligheid, interesse en de goede sfeer. Peter, ik ben blij dat je mij destijds als eerste op het spoor van "toch gaan promoveren" hebt gezet, want ik had de grenzen van mijn kunnen toen nog lang niet ontdekt. Dat heb ik misschien nog niet helemaal, maar ik weet nu in ieder geval wel hoe het voelt om trots te zijn op iets dat niet "vanzelf" gaat. Ik kan hier niet iedereen afzonderlijk bedanken, maar lieve Tarquinia, je was een hele fijne kamergenoot: een fantastisch klankbord, een luisterend oor, superattent en als ik even afleiding nodig had zorgde jij voor het nodige snufje bijzondere humor! Je kon altijd precies aan me zien wanneer je tegen me kon praten of wanneer je beter je mond kon houden. Ik ben erg blij dat je mij als paranimf wilt begeleiden en dat je de foto voor de omslag hebt willen maken. En ... oh ja ... het jackpot bonusgetal is 12 , en ik weet zeker dat je gewonnen hebt!

Onmisbaar bij het zonder al te veel stress schrijven van mijn proefschrift waren uiteraard ook de mensen in mijn privé-omgeving. Mam en pap, door mij op te voeden in een veilige haven, met een optimistische no-nonsens inslag, hebben jullie de basis 
gelegd voor mijn persoontje, dat zich niet gek laat maken en in een rechte lijn vol vertrouwen op haar doelen afgaat. En wat ontspant er beter dan bij mensen zijn waar je volledig jezelf kunt zijn. Ireen, Anja, Ay Lin, Ellen, Ilse, Judith en Marieke: Ik hou van jullie ;-). Ay, fijn dat jij als mijn paranimf deze groep mensen wilt vertegenwoordigen. Opa, nichtjes en andere (schoon)familie, George en Esther, "Golf"-vrienden, BeNdeleden, en "plussen" ik wil jullie bedanken voor de gezelligheid en belangstelling.

En last but not least, Arno, ik ga je niet bedanken voor een intensief stimuleren om dit tot een goed einde te brengen :-) wel voor je nuchtere visie die neerkomt op "doe vooral waar je gelukkig van wordt" en voor jouw belangrijke aandeel dáárin, en natuurlijk voor Odto! 


\section{About the author}

Dewi Segaar was born on March 21st, 1978 in Amsterdam. She graduated from secondary school in 1996. Between 1996 and 2001 she studied Human Nutrition at the Wageningen University. Her graduation projects were on the influence of the visual packing on the taste-experience of provisions and on the marketing opportunities for eel. In March 2001, she received her MSc degree, after which she started to work at STIVORO on a 2-year long research project about smoking at the workplace. Thereafter, she was employed as a junior researcher at the department of Health Promotion and Health Education of the Maastricht University and performed the research that formed the basis for this thesis. Since januari 2005 she also participates in a knowledge centre that provides information to support professionals with the implementation of the Dutch national clinical guideline for smoking cessation support. 